Morin, Alexandre J. S.; Arens, A. Katrin; Marsh, Herbert W.

\title{
A bifactor exploratory structural equation modeling framework for the identification of distinct sources of construct-relevant psychometric multidimensionality
}

formal und inhaltlich überarbeitete Version der Originalveröffentlichung in:

formally and content revised edition of the original source in:

Structural equation modeling 23 (2016) 1, S. 116-139, 10.1080/10705511.2014.961800

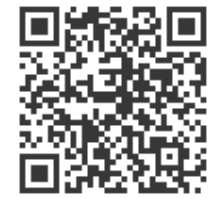

Bitte verwenden Sie in der Quellenangabe folgende URN oder DOI /

Please use the following URN or DOI for reference:

urn:nbn:de:0111-dipfdocs-201721

10.25657/02:20172

https://nbn-resolving.org/urn:nbn:de:0111-dipfdocs-201721

https://doi.org/10.25657/02:20172

\section{Nutzungsbedingungen}

Gewährt wird ein nicht exklusives, nicht übertragbares, persönliches und beschränktes Recht auf Nutzung dieses Dokuments. Dieses Dokument is ausschließlich für den persönlichen, nicht-kommerziellen Gebrauch bestimmt. Die Nutzung stellt keine Übertragung des Eigentumsrechts an diesem Dokument dar und gilt vorbehaltlich der folgenden Einschränkungen Auf sämtlichen Kopien dieses Dokuments müssen alle Urheberrechtshinweise und sonstigen Hinweise auf gesetzlichen Schutz beibehalten werden. Sie dürfen dieses Dokument nicht in irgendeiner Weise abändern, noch dürfen Sie dieses Dokument für öffentliche oder abändern, noch dürfen Sie dieses Dokument für öffentliche oder
kommerzielle Zwecke vervielfältigen, öffentlich ausstellen, aufführen, vertreiben oder anderweitig nutzen.

Mit der Verwendung dieses Dokuments erkennen Sie die Nutzungsbedingungen an.

\section{Terms of use}

We grant a non-exclusive, non-transferable, individual and limited right to using this document.

This document is solely intended for your personal, non-commercial use. Use of this document does not include any transfer of property rights and it is conditional to the following limitations: All of the copies of this documents must retain all copyright information and other information regarding legal protection. You are not allowed to alter this document in any way, to copy it for

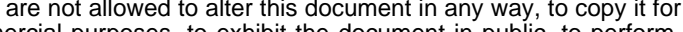
public or commercial purposes, to exhibit the document in public, to perform,
distribute or otherwise use the document in public.

By using this particular document, you accept the above-stated conditions of use.

\section{Kontakt / Contact:}

DIPF | Leibniz-Institut für

Bildungsforschung und Bildungsinformation

Frankfurter Forschungsbibliothek

publikationen@dipf.de

www.dipfdocs.de

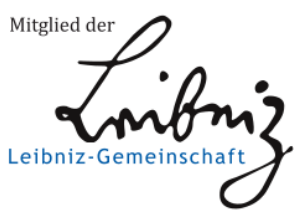


This is an Accepted Manuscript of an article published by Taylor \& Francis in Structural equation modeling on 18/06/2015, available online: http://www.tandfonline.com/10.1080/10705511.2014.961800. 
Running head: Construct-Relevant Multidimensionality

\section{A Bifactor Exploratory Structural Equation Modeling Framework for the Identification of Distinct Sources of Construct-Relevant Psychometric Multidimensionality}

Alexandre J.S. Morin*, Institute for Positive Psychology and Education, Australian Catholic University

A. Katrin Arens*, German Institute for International Educational Research

Herbert W. Marsh, Institute for Positive Psychology and Education, Australian Catholic University, Oxford University, King Saud University

* The first two authors (A.J.S.M. \& A.K.A.) contributed equally to this article and their order was determined at random: both should thus be considered first authors.

This is the prepublication version of the following manuscript:

Morin, A.J.S., Arens, A.K., \& Marsh, H.W. (2016). A bifactor exploratory structural equation modeling framework for the identification of distinct sources of construct-relevant psychometric multidimensionality. Structural Equation Modeling: A Multidisciplinary Journal, 23, 116-139. http://doi.org/10.1080/10705511.2014.961800

This paper is not the copy of record and may not exactly replicate the authoritative document published in Structural Equation Modeling: A Multidisciplinary Journal.

\section{Acknowledgements}

This article was prepared when the second author was a visiting scholar at the Institute for Positive Psychology and Education, Australia. The research was funded by a scholarship of the German Academic Exchange Service (DAAD) to the second author. This research was also made possible by grants from the Australian Research Council (DP130102713; DP140101559). 


\begin{abstract}
This study aims to illustrate an overarching psychometric approach of broad relevance to investigations of two sources of construct-relevant psychometric multidimensionality present in many complex multidimensional instruments that are routinely used in psychological and educational research. These two sources of construct-relevant psychometric multidimensionality are related to: (a) the fallible nature of indicators as perfect indicators of a single construct; (b) the hierarchical nature of the constructs being assessed. The first source is identified by comparing confirmatory factor analytic (CFA) and exploratory structural equation modeling (ESEM) solutions. The second source is identified by comparing first-order, hierarchical, and bifactor measurement models. To provide an applied illustration of the substantive relevance of this framework, we first apply these models to a sample of German children $(N=1957)$ who completed the Self-Description Questionnaire (SDQ-I). Then, in a second study using a simulated data set, we provide a more pedagogical illustration of the proposed framework and the broad range of possible applications of bifactor-ESEM models.
\end{abstract}

Key words: Psychometric, multidimensionality, confirmatory factor analyses (CFA), and exploratory structural equation modelling (ESEM), hierarchical, bifactor, self-concept. 
This manuscript presents an overarching approach that has broad relevance to investigations of multidimensional instruments. More specifically, we illustrate the use of the emerging Exploratory Structural Equation Modeling (ESEM) framework, of more traditional bifactor models, and of their combination in bifactor-ESEM. This combined framework is presented as a way to fully explore the mechanisms underlying sources of construct-relevant psychometric multidimensionality present in complex measurement instruments. We provide a substantive illustration of the meaning of these sources of construct-relevant psychometric multidimensionality modeled as part of this overarching framework using real data on the preadolescent version of the Self-Description Questionnaire (SDQ-I; Marsh, 1990). Then we illustrate how to conduct these analyses using a simpler simulated data set.

\section{Old, New, and "Rediscovered" Approaches to Multidimensionality}

For decades, the typical approach to the analysis of multidimensional instruments has been based on confirmatory factor analyses (CFA). It is hard to downplay the impact that CFA and the overarching Structural Equation Modeling (SEM) framework have had on psychological and educational research (e.g., Bollen, 1989; Jöreskog, 1973). SEM provides the possibility to rely on a confirmatory approach to psychometric measurement, allowing for the systematic comparison of alternative a priori representations of the data based on systematic fit assessment procedures, and to estimate relations between latent constructs corrected for measurement errors. These advances were so major that it is not surprising that within a decade CFA almost completely supplanted classical approaches such as exploratory factor analyses (EFA). However, CFA relies on the highly restrictive Independent Cluster Model (ICM), in which cross-loadings between items and non-target factors are assumed to be exactly zero. It was recently observed that instruments assessing multidimensional constructs seldom manage to achieve reasonable fit within the ICM-CFA framework (Marsh, Lüdtke et al., 2010; Marsh et al., 2009; McCrae, Zonderman, Costa, Bond, \& Paunonen, 1996). In answer to this observation, more flexible approaches have been proposed (Asparouhov \& Muthén, 2009; Morin, Marsh, \& Nagengast, 2013), or "rediscovered" (Reise, 2012), such as ESEM, bifactor models, and their combination. These approaches, described below, arguably provide a better representation of complex multidimensional structures without relying on unrealistic ICM assumptions. In the upcoming pages, we argue that ICM-CFA models typically fail to account for at least two sources of 
construct-relevant psychometric multidimensionality, and may thus produce biased parameter estimates as a result of this limitation. Before presenting these two sources, it is important to differentiate substantive multidimensionality, which refers to instruments that have been specifically designed to assess multiple dimensions with separate items tapping into each of these dimensions, and psychometric multidimensionality, which refers to the idea that the items forming an instrument may be associated with more than one source of true score variance (i.e., be associated with more than one content area). In many multidimensional instruments, two sources of construct-relevant psychometric multidimensionality are likely to be present and related to: (a) the hierarchical nature of the constructs being assessed whereby all items may be expected to present a significant level of association with their own subscales (e.g., peer self-concept, verbal intelligence, or attention difficulties), as well as hierarchically-superior constructs (e.g., global self-esteem, global intelligence, or attention deficit/hyperactivity disorders); (b) the fallible nature of indicators typically used to measure psychological and educational constructs, which tends to be reinforced in instruments assessing conceptually-related and partially overlapping domains (i.e., such as peer and parent self-concepts, verbal intelligence and memory, or attention difficulty and impulsivity). We focus on these two sources of construct-relevant psychometric multidimensionality whereby items may present associations with multiple hierarchically-superior or substantively-related constructs. Additionally, as shown in our first study, construct-irrelevant psychometric multidimensionality (due to item wording, method effects, etc.) may also be present and can easily be controlled through the inclusion of method factors (Eid et al., 2008; Marsh, Scalas, \& Nagengast, 2010).

\section{Psychometric Multidimensionality due to the Co-Existence of Global and Specific Constructs}

A first source of psychometric multidimensionality is related to the possibility that the items used to assess the multiple dimensions included in an instrument could reflect multiple hierarchicallyorganized constructs: Their own specific subscale, as well as more global constructs. A classical solution to this issue is provided by hierarchical (i.e., higher-order) CFA. In hierarchical CFA, each item is specified as loading on its specific subscale (a first-order factor), and each first-order factor is specified as loading on a higher-order factor (e.g., Rindskopf \& Rose, 1988).

Bifactor models provide an alternative to hierarchical models (Chen, West, \& Sousa, 2006; 
Holzinger \& Swineford, 1937; Reise, Moore, \& Haviland, 2010). For illustrative purposes, an ICMCFA, a hierarchical-CFA, and a bifactor-CFA model are presented on the left side of Figure 1. A bifactor model is based on the assumption that a $f$-factor solution exists for a set of $n$ items with one Global (G) factor and $f$-1 Specific (S) factors (also called group factors). The items' loadings on the G-factor and on one of $f-1$ substantive S-factors are estimated while other loadings are constrained to be zero; although these models may also incorporate additional method factors. All factors are set to be orthogonal (i.e., the correlations between the S-factors and between the S-factors and the G-factor are all constrained to be zero). This model partitions the total covariance among the items into a $\mathrm{G}$ component underlying all items, and $f-1 \mathrm{~S}$ components explaining the residual covariance not explained by the G-factor. Bifactor models are well established in research on intelligence (e.g., Holzinger \& Swineford, 1937; Gignac \& Watkins, 2013), and have also been successfully applied to noncognitive constructs such as quality of life (e.g., Reise, Morizot, \& Hays, 2007), attention disorders (e.g., Caci, Morin, \& Tran, 2013; Morin, Tran, \& Caci, 2013), or mood and anxiety disorders (e.g., Gignac, Palmer, \& Stough, 2007; Simms, Grös, Watson, \& O’Hara, 2008). A bifactor model directly tests whether a global construct, reflected through the G-factor, exists as a unitary dimension underlying the answers to all items and co-exists with multiple more specific facets (Sfactors) defined by the part of the items that is unexplained by the G-factor. Thus, both hierarchical and bifactor models assume that there exists a global construct underlying answers to all items included in an instrument, whereas ICM-CFA simply assumes distinct facets without a common core.

Similarities have been noted between hierarchical and bifactor models, which both test for the presence of global and specific dimensions underlying the responses to multiple items. These similarities are related to the possibility of applying a Schmid and Leiman (1957) transformation procedure (SLP) to a hierarchical model in order to convert it to a bifactor approximation. However, the SLP makes obvious that hierarchical models implicitly rely on far more stringent assumptions than bifactor models (Chen et al., 2006; Jenrich \& Bentler, 2011; Reise, 2012). In particular, when a SLP is applied to a hierarchical model, the relation between an item and the G-factor from the bifactor approximation is represented as the indirect effect of the higher-order factor on the item, as 'mediated' by the first-order factor. More precisely, each item's first-order factor loading is multiplied 
by the loading of this first-order factor on the second-order factor, which in turns yields the loadings of this item on the SLP-estimated G-factor. The second term in this multiplication is thus a constant as far as the items associated with a single first-order factor are concerned. Similarly, the relations between the items and the SLP-estimated S-factors are reflected by the product of their loadings on their first-order factor by the squared root of the disturbance of this first-order factor (corresponding to the regression path associated with the unique part of the first-order factor). This second term is also a constant and reflects the unique part of the first-order factor that remains unexplained by the higher-order factor (for worked examples, see Gignac, 2007; Jenrich \& Bentler, 2011; Reise, 2012). The SLP makes explicit that higher-order models rely on stringent proportionality constraints: Each item's association with the SLP G-factor and S-factors are obtained by multiplying their first-order loadings by constants. These constraints imply that the ratio of G-factor to S-factors loadings for all items associated with the same first-order dimension will be exactly the same. Although these constraints may hold under specific conditions, they are unlikely to hold in real-world settings involving complex instruments (Reise, 2012; Yung, Thissen, \& McLeod, 1999). These constraints are one reason why true bifactor models tend to provide a much better fit to the data than hierarchical models (Brunner, Nagy, \& Wilhelm, 2012; Chen et al., 2006; Reise, 2012; but also see Murray \& Johnson, 2013). Furthermore, Jenrich and Bentler (2011) demonstrated that, when the population model underlying the data corresponds to a bifactor model without meeting the SLP proportionality constraints, the SLP generally fails to recover the underlying bifactor structure of the data.

\section{Psychometric Multidimensionality due to the Fallible Nature of Indicators}

A second source of construct-relevant psychometric multidimensionality that is typically neglected within the traditional ICM-CFA framework is that items are very seldom perfectly pure indicators of the constructs they are purported to measure. Rather, they tend to be fallible indicators including at least some degree of relevant association with constructs other than the main constructs that they are designed to measure. More precisely, items are known to incorporate a part of random measurement error, which is traditionally assessed as part of reliability analyses and modeled as part of the items' uniquenesses in EFA or CFA. However, items also tend to present some degree of systematic association with other constructs (a form of measurement error usually assessed as part of 
validity analyses) that is typically expressed through cross-loadings in EFA but is constrained to be zero in ICM-CFA. Although not limited to this context, this phenomenon tends to be reinforced when the instruments includes multiple factors related to conceptually-related and partially overlapping domains. Particularly in these contexts, ICM assumptions might be unrealistically restrictive. Still, no matter the content of the instrument that is considered, most indicators are likely to be imperfect to some extent and thus present at least some level of systematic associations with other constructs.

This reality is made worse when the instrument also includes items designed to directly assess hierarchically-superior constructs (e.g., global self-esteem, intelligence, or externalizing behaviors) usually specified as separate subscales which should also logically present direct associations with hierarchically inferior items/subscales (e.g., math self-concept, memory, impulsivity). In the absence of a bifactor model specifically taking hierarchical relations into account, cross-loadings are to be expected as a way to reflect these hierarchically-superior constructs. However, even in a bifactor model taking hierarchically-superior constructs into account, cross-loadings can still be expected due to the fallibility of indicators, particularly in the presence of partially overlapping domains (e.g., peer and parent self-concepts, verbal intelligence and memory, impulsivity and attention difficulties).

When real cross-loadings are forced to be zero in ICM-CFA, the only way for them to be expressed is through the inflation of the estimated factor correlations. Indeed, even when the ICMCFA model fits well in the first place (see Marsh, Liem, Martin, Morin, \& Nagengast, 2011; Marsh, Nagengast et al., 2011), factor correlations will typically be at least somewhat inflated unless all cross-loadings are close to zero. Interestingly, simulations studies showed that EFA usually results in more exact estimates of the true population values for the latent factor correlations than CFA (Asparouhov \& Muthén, 2009; Marsh, Lüdtke, Nagengast, Morin, \& Von Davier, 2013; Schmitt \& Sass, 2011). Even when the true population model corresponds to ICM-CFA assumptions, EFA still results in unbiased parameter estimates. These observations seem to argue in favor of EFA as providing a more realistic and flexible measurement model for multidimensional instruments. Unfortunately, EFA has been superseded by the methodological advances associated with CFA/SEM and by the erroneous assumption that EFA was unsuitable to confirmatory studies. However: 
CFA is that all cross loadings are freely estimated in EFA. Due to this free estimation of all cross loadings, EFA is clearly more naturally suited to exploration than CFA. However, statistically, nothing precludes the use of EFA for confirmatory purposes (Morin, Marsh et al., 2013, p. 396). Asparouhov and Muthén (2009) recently developed ESEM, which allows for the integration of EFA within the overarching SEM framework, making methodological advances typically reserved to CFA/SEM available for EFA measurement models (Marsh, Morin, Parker, \& Kaur, 2014; Marsh et al., 2009; Morin, Marsh et al., 2013). Further, when ESEM is estimated with target rotation, it becomes possible to specify a priori hypotheses regarding the expected factor structure and thus to use ESEM for purely confirmatory purposes (Asparouhov \& Muthén, 2009; Browne, 2001).

\section{An Integrated Test of Multidimensionality}

A comprehensive test of the structure of many multidimensional measures apparently requires the consideration of the two sources of construct-relevant psychometric multidimensionality described above. The assessment of a hierarchically-organized construct, especially when coupled with the inclusion of subscales specifically designed to represent the global construct of interest, would typically argue in favor of bifactor or hierarchical models. However, both bifactor and hierarchical models typically neglect item cross-loadings due to the fallible nature of indicators as providing a reflection of one, and only one, construct, which are likely to be expressed through the inflation of the variance attributed to the G-factor (e.g., Murray, \& Johnson, 2013). These expected cross-loadings thus apparently argue in favor of ESEM. However, a first-order ESEM model will likely ignore the presence of hierarchically-superior constructs, which will end up being expressed through inflated cross-loadings. In sum, it appears that a bifactor-ESEM or a hierarchical-ESEM may be needed to fully capture the hierarchical and multidimensional nature of instruments incorporating both sources of construct-relevant psychometric multidimensionality.

Unfortunately, it has typically not been possible to combine these two methodological approaches into a single model. For instance, hierarchical models have generally been specified within the CFA framework. The estimation of hierarchical ESEM models needs to rely on suboptimal two-step procedures where correlations among the first-order factors are used to estimate the higherorder factor. This leads to higher-order factors that are a simple re-expression (an equivalent model) 
of the first-order correlations (for recent illustrations, see Meleddu, Guicciardi, Scalas, \& Fada, 2012; Reise, 2012). Similarly, the estimation of bifactor models has typically been limited to CFA.

However, recent developments have made these combinations possible. Morin, Marsh et al. (2013; also see Marsh et al., 2014; Marsh, Nagengast, \& Morin, 2013) recently proposed ESEMWithin-CFA, allowing a specific first-order ESEM solution to be re-expressed using CFA. This method allows for tests of hierarchical models where the first-order structure replicates the ESEM solution (with the same constraints, degrees of freedom, fit, and parameter estimates), while allowing for the estimation of a higher-order factor defined from first-order ESEM factors. Similarly, bifactor rotations (Jennrich \& Bentler, 2011, 2012), including a bifactor target rotation that can be used to express clear a priori hypotheses (Reise, 2012; Reise, Moore, \& Maydeu-Olivares, 2011), have recently been developed within the EFA/ESEM framework. This development allows for the direct estimation of true bifactor-ESEM models. For illustrative purposes, an ESEM, a hierarchical-ESEM, and a bifactor-ESEM are presented on the right side of Figure 1. These developments provide an overarching framework for the systematic investigation of these two sources of construct-relevant psychometric multidimensionality likely to be present in many complex psychometric measures.

To illustrate this integrative framework, we rely on two studies. The first study provides an applied illustration of the substantive relevance of the various models considered here using a real data set of German children who completed the SDQ-I (Marsh, 1990). After discussing why both sources of construct-relevant multidimensionality are likely to be present in this instrument, we illustrate the use of the proposed framework, and further show the flexibility of bifactor-ESEM by presenting detailed tests of measurement invariance of the final model across gender. Although we provide the input codes used in these analyses at the end of the online supplements, they may be too complex to properly serve as pedagogical material for readers less familiar with Mplus. We thus conducted a second study using a simpler simulated data set and a complete set of pedagogicallyannotated input files, including those used to simulate the data in the first place so as to provide readers with the data set for practice purposes. Furthermore, this second study provides a more extensive set of illustrations, including multiple group tests of measurement invariance, Multiple Indicator Multiple Causes (MIMIC) models, as well as a predictive mediation model. 


\section{Study 1: Substantive Illustration}

In this study, we contrast alternative representations of the SDQ-I (ICM-CFA, hierarchicalCFA, bifactor-CFA, ESEM, hierarchical-ESEM, and bifactor-ESEM) to illustrate how these methods allow us to achieve a clearer understanding of the sources of construct-relevant multidimensionality potentially at play in this instrument. Although our goal is mainly to illustrate this methodological framework, we reinforce that no analysis should be conducted in disconnection from substantive theory and expectations. Thus, we do not argue that this framework should be blindly applied to the study of any psychometric measure. Rather, we argue that this framework would bring valuable information to the analysis of psychometric measures for which previous results and substantive theory suggest that sources of construct-relevant multidimensionality might be present. With this in mind, we selected the SDQ-I, a well-known instrument (Byrne, 1996; Marsh, 1990, 2007) likely to include both sources of construct-relevant psychometric multidimensionality.

The SDQ-I is based on Shavelson, Hubner, and Stanton (1976) seminal hierarchical and multidimensional model of self-concept. This hierarchical structure is further reinforced in the SDQ-I through the inclusion of scales directly assessing hierarchically superior constructs (i.e., global selfesteem and general academic self-concept). Although previous studies failed to support a strong higher-order factor structure for multidimensional self-concept measures (e.g., Abu-Hilal \& AalHussain, 1997; Marsh \& Hocevar, 1985), Marsh (1987) showed that global self-concept defined as a higher-order factor and global self-concept (i.e., global self-esteem) directly assessed from a separate scale were highly correlated with one another. Similarly, general academic self-concept was found to share high positive relations with math and verbal self-concepts even though these two self-concepts are almost uncorrelated - or even negatively related - to one another (Möller, Pohlmann, Köller, \& Marsh, 2009). In fact, Brunner et al. (Brunner, Keller, Hornung, Reichert, \& Martin, 2009; Brunner, Lüdtke, \& Trautwein, 2008; Brunner et al., 2010) showed that a bifactor model provided better fit to the data than a corresponding CFA model when applied to academic self-concept measures. These results clearly support the interest of testing a bifactor representation of the SDQ-I to model constructrelevant multidimensionality due to the presence of hierarchically-superior constructs.

However, the SDQ-I is also inherently multidimensional and taps into conceptually-related 
and partially overlapping constructs (e.g., physical appearance and physical abilty self-concept). Although ICM-CFA correlations between the SDQ-I factors tend to remain reasonably small (typically $\leq .50$; e.g., Arens, Yeung, Craven, \& Hasselhorn, 2013; Marsh \& Ayotte, 2003), this does not mean that they are not somehow inflated due to the elimination of potentially meaningful crossloadings. Indeed, most previous EFA investigations of the SDQ-I revealed multiple cross-loadings (Watkins \& Akande, 1992; Watkins \& Dong, 1994; Watkins, Juhasz, Walker, \& Janvlaitiene, 1995). Morin and Maïano (2011) recently applied ESEM to the Physical Self Inventory (PSI), an instrument assessing multidimensional physical self-conceptions. Their results showed the superiority of ESEM over ICM-CFA, and revealed multiple cross-loadings, most of which proved to be substantively meaningful. These results support the interest of applying ESEM to the SDQ-I to model constructrelevant multidimensionality due to the fallible nature of indicators.

\section{Method}

The present study relies on a sample of German students $(N=1957 ; 50.5 \%$ boys $)$ attending grades 3 to 6 in mixed-gender public schools. These students are aged between 7 and15 years $(M=$ 10.66; $S D=1.30$ ), and all obtained parental consent for participation in the study. The German version of the SDQ-I (Arens et al., 2013) was administered to all participants during regular school lessons following standardized administration guidelines relying on a read-aloud procedure (Byrne, 1996; Marsh, 1990). The German SDQ-I consist of 11 subscales: physical appearance ( 9 items; $\alpha=$ .884 ), physical ability ( 9 items; $\alpha=.894$ ), peer relations ( 9 items; $\alpha=.861$ ), parent relations ( 9 items; $\alpha=.861$ ), math competence ( 5 items; $\alpha=.928$ ), math affect ( 5 items; $\alpha=.943$ ), German competence (5 items; $\alpha=.907$ ), German affect ( 5 items; $\alpha=.919$ ), general academic competence ( 5 items; $\alpha=$ .827), general academic affect ( 5 items; $\alpha=.858$ ), and global self-esteem (10 items; $\alpha=.853$ ). The latter directly assesses global self-concept, whereas the general academic competence and affect subscales both assess academic self-concept across all school subjects. Each of the SDQ-I items are rated on a 5-point Likert scale (false, mostly false, sometimes true/sometimes false, mostly true, true). A complete list of the items included in the English and German SDQ-I is available at: http://www.acu.edu.au/ippe/.

\section{Analyses}


Alternative Models. All analyses were conducted with Mplus 7.11 (Muthén \& Muthén, 19982013), based on the robust maximum likelihood (MLR) estimator providing standard errors and fit indices that are robust to the Likert nature of the items and violations of normality assumptions. Full Information robust Maximum Likelihood (FIML) estimation was used to handle the small amount of missing data at the item level $(M=0.646 \%$; Enders, 2010; Graham, 2009). We first contrasted ICMCFA, hierarchical-CFA (H-CFA), bifactor-CFA (B-CFA), ESEM, hierarchical ESEM (H-ESEM), and bifactor-ESEM (B-ESEM) representations of the underlying structure of the answers provided to the full SDQ-I (see Figure 1 for simplified illustrations of these models). In the ICM-CFA model, each item was only allowed to load on the factor it was assumed to measure and no cross-loadings on other self-concept factors were allowed. This model included 11 correlated factors representing the previously described SDQ-I subscales. In the H-CFA model, these 11 factors were specified as being related to a single higher-order CFA factor, with no residual correlations specified between the 11 first-order factors. In the B-CFA model, all items were allowed to simultaneously load on one Gfactor and on $11 \mathrm{~S}$-factors corresponding to the a priori self-concept factors measured by the SDQ-I, with no cross-loadings allowed across S-factors. The G-factor and all S-factors were specified as orthogonal in order to ensure the interpretability of the solution in line with bifactor assumptions that the S-factors reflect the part of the items' variance that is not explained by the G-factor, while the Gfactor reflects the part of the items variance that is shared across all items (e.g., Chen et al., 2006; Reise, 2012). Then, these models were first contrasted with an 11-factor ESEM representation of the SDQ-I estimated based on oblique target rotation (Asparouhov \& Muthén, 2009; Browne, 2001). Target rotation seemed particularly appropriate as it allows for the pre-specification of target and nontarget factor loadings in a confirmatory manner. According to the most common specification of target rotation, all cross-loadings were "targeted" to be close to zero, while all of the main loadings were freely estimated. An H-ESEM model was then estimated from this model using ESEM-WithinCFA (Morin, Marsh et al., 2013). In this model, all 11 first-order factors were specified as related to a single higher-order factor, with no residual correlations between the 11 first-order factors. Finally, a B-ESEM model was estimated in line with typical bifactor assumptions using orthogonal bi-factor target rotation (Reise, 2012; Reise et al., 2011), which ensured comparability with the B-CFA ${ }^{1}$. In this 
model, all items were allowed to define a G-factor, while the 11 S-factors were defined from the same pattern of target and non-target factor loadings that was used in the first-order ESEM solution.

Construct-Irrelevant Multidimensionality. The SDQ-I includes a total of 12 negatively worded items (items 6, 12, 17, 21, 23, 30, 33, 37, 47, 61, 65, and 75, italicized in Table 1), which were reversed coded prior to the analyses to facilitate interpretation. To take into account the methodological artifact due to the wording of these items (i.e., construct-irrelevant psychometric multidimensionality), all models included a method factor underlying all negatively-worded items (e.g., Marsh, Scalas et al., 2010). In line with typical specifications of method factors and to ensure that all models remained comparable, this method factor was modeled as an orthogonal CFA factor defined strictly through the negatively-worded items. Furthermore, the items used to assess the various academic subscales are strictly parallel (e.g., "I am good at Math"; "I am good at German"; "I am good at all school subjects"). Thus, a priori correlated uniquenesses among matching indicators of the academic subscales were also included to the models. This inclusion reflects the idea that the unique variance of these items (i.e., uniquenesses, reflecting construct-irrelevant sources of influences and random error) is likely to be shared among items with parallel wordings (i.e., due to convergent sources of construct-irrelevant influence; Marsh, 2007; Marsh, Abduljabbar et al., 2013).

Generally, the inclusion of ex post facto correlated uniquenesses as a way to improve model fit should be avoided and has been labeled as a "disaster" for research (Schweizer, 2012, p.1). Even when legitimate a priori controls are required (such as in the present study), method factors should be preferred to correlated uniquenesses. As noted by Schweizer (2012), method factors explicitly estimate construct-irrelevant sources of variance, whereas correlated uniquenesses simply partial them out - bringing no new information to the model. In this study, it was not realistic to include ten additional method factors reflecting the parallel wording of the items used to assess the academic subscales (i.e., five items per academic competence subscale, all with parallel wording, and five items per academic affect subscale, also with parallel wording). However, parallel wording is more naturally suited to correlated uniquenesses than negative wording. Furthermore, this provides an occasion to illustrate the implementation of both forms of control in the proposed framework.

The control of these sources of construct-irrelevant psychometric multidimensionality is 
particularly important in the application of the integrative framework proposed here. Indeed, Murray and Johnson (2013) recently showed that bifactor models (the same argument applies to ESEM) are particularly efficient at absorbing unmodeled complexity (e.g., correlated uniquenesses, crossloadings), which may in turn inflate the fit of these models relative to models not taking this complexity into account. The inclusion of these methodological controls of a priori method effects, as well as the comparison of ESEM and CFA, and bifactor and non-bifactor models, allow us to control for this possibility. All models including these a priori methodological controls systematically provided a better fit to the data than models without them. However, including these controls had no impact on the results or the final model selection (see Table S1 in the online supplements).

Measurement Invariance. The measurement invariance across gender of the final retained model was then investigated (Meredith, 1993; Millsap, 2011). In the least restrictive model (configural invariance), the same pattern of associations between items and factors, and the same number of factors, were estimated for males and females with no added equality constraints. A second model in which all factor loadings (and cross-loadings) on the substantive and methodological factors were constrained to be invariant across groups (weak measurement invariance) was then estimated. This model is an essential prerequisite to any form of gender-based comparison based on the SDQ-I. In the third step, a model where both the factor loadings and items' intercepts were constrained to be invariant across groups (strong measurement invariance) was estimated. This model represents a prerequisite to valid latent means comparisons across groups. A fourth model in which the factor loadings, items' intercepts and items' uniquenesses were constrained to be invariant across groups (strict measurement invariance) was estimated. Although not a requirement for the present study where comparisons are based on latent variables, this steps is an essential prerequisite to gender-based comparisons based on manifest (aggregated) scale scores. Then, to ensure that the measurement model was indeed fully invariant across groups, we also verified whether the correlated uniquenesses included between the parallel-worded items for the academic subscales were also invariant across groups. Two additional steps were tested in which further invariance constraints were specified at the level of the factor variances/covariances and latent means in order to further investigate possible gender-based differences in the association between self-concepts facets, variability, and latent means. 
For more details, the reader is referred to Morin, Marsh et al. (2013) and Millsap (2011).

Model Evaluation. Given the known oversensitivity of the chi-square test of exact fit and of chi-square differences tests to sample size and minor model misspecifications (e.g., Marsh, Hau, \& Grayson, 2005), we relied on common goodness-of-fit indices and information criteria to describe the fit of the alternative models: the comparative fit index (CFI; Bentler, 1990), the Tucker-Lewis index (TLI; Tucker \& Lewis, 1973), the root mean square error of approximation (RMSEA; Steiger, 1990) with its confidence interval, the Akaike Information Criteria (AIC; Akaike, 1987), the Constant AIC (CAIC; Bozdogan, 1987), the Bayesian Information Criteria (BIC; Schwartz, 1978), and the samplesize adjusted BIC (ABIC; Sclove, 1987). According to typical interpretation guidelines (e.g., Browne \& Cudeck, 1993; Hu \& Bentler, 1999; Marsh, Hau, \& Wen, 2004; Marsh et al., 2005), values greater than .90 and .95 for the CFI and TLI are considered to be respectively indicative of adequate and excellent fit to the data, while values smaller than .08 or .06 for the RMSEA support respectively acceptable and excellent model fit. Similarly, in comparing nested models forming, for instance, the sequence of invariance tests, common guidelines (Chen, 2007; Cheung \& Rensvold, 2002) suggest that models can be seen as providing a similar degree of fit to the data (thus supporting the adequacy of invariance constraints) as long as decreases in CFI remain under .01 and increases in RMSEA remain under .015 between less restrictive and more restrictive models. It has also been suggested to complement this information by the examination of changes in TLI (with guidelines similar to those for CFI) that may be useful with complex models due to the incorporation of a penalty for parsimony (Marsh et al., 2009; Morin, Marsh et al., 2013). As articulated by Cheung and Lau (2012, p. 169) "One pitfall of this approach is that the $\triangle$ CFI has no known sampling distribution and, hence, is not subject to any significance testing. These cutoff values may thus be criticized as arbitrary." Although the information criteria (AIC, CAIC, BIC, ABIC) do not, in and of themselves, describe the fit of a model, a lower value reflects a better fit to the data of one model in comparison to a model with higher values so that in a set of nested models the best fitting model is the one with the lowest value.

It is important to note that these descriptive guidelines have so far been established for CFA. Although previous ESEM applications have generally relied on similar criteria (e.g., Marsh et al., 2009; Morin, Marsh et al., 2013; also see Grimm, Steele, Ram, \& Nesselroade, 2013), their adequacy 
for ESEM still has to be more thoroughly investigated. In this regard, it has been suggested that indicators including a correction for parsimony (i.e., TLI, RMSEA, AIC, CAIC, BIC, ABIC) might be particularly important in ESEM given that the total number of estimated parameters is typically much larger than in CFA (Marsh, Lüdtke et al., 2010; Marsh et al., 2009; Morin, Marsh et al., 2013). Furthermore, although the efficacy of the proposed descriptive guidelines for the comparison of nested invariance models has been validated in CFA for tests of weak, strong, and strict measurement invariance (Chen, 2007; Cheung \& Rensvold, 2002), they appear to be of questionable efficacy for tests of latent mean invariance (Fan \& Sivo, 2009). In addition, these indices still appear to show sensitivity to design conditions and model complexity (e.g., Fan \& Sivo, 2005, 2007), calling into question the generalizability of these guidelines outside of the conditions considered in previous simulation studies and, importantly, the CFA framework. Although information criteria (AIC, CAIC, BIC, ABIC) appear to represent a less "subjective" alternative, their known dependency to sample size creates a confounding: Given a sufficiently large sample size, these indicators will always support more complex alternatives (see Marsh et al., 2005). In sum, all of these interpretation guidelines (be they related to goodness-of-fit indices or information criteria) should not be treated as "golden rules" or used for inferential purposes, but only as rough guidelines for descriptive model evaluation and comparison that should also take into account parameters estimates, statistical conformity and theoretical adequacy (Fan \& Sivo, 2009; Marsh et al., 2004, 2005). This is also the approach generally advocated in ESEM (e.g., Grimm et al., 2013; Marsh et al., 2009; Morin, Marsh et al., 2013).

\section{Results}

Table 1 (top section) presents the goodness-of-fit indices and information criteria associated with the models. The ICM-CFA solution $(\mathrm{CFI}=.921$; TLI $=.916$; $\mathrm{RMSEA}=.033)$ provides an acceptable degree of fit to the data, whereas both the H-CFA and the B-CFA appear to be suboptimal in terms of fit (CFI and TLI $<.90$ and higher values on the information criteria). The ESEM solution provides an acceptable $(\mathrm{TLI}=.947)$ to excellent $(\mathrm{CFI}=.963$; RMSEA $=.026)$ degree of fit to the data, and an apparently better representation of the data than the ICM-CFA model according to improvement in fit indices and a decrease in the values of the AIC and ABIC. The B-ESEM model provides an excellent degree of fit to the data according to all indices $(\mathrm{CFI}=.970 ; \mathrm{TLI}=.956$; 
RMSEA $=.024)$, and a slightly better level of fit to the data and lower values for the information criteria than all other models. The more rigid H-ESEM does not fit the data as well as either ESEM or the B-ESEM (higher information criteria, lower fit indices). Based on this information, the B-ESEM model appears to provide the best representation of the data. However, as mentioned before, this information on model fit should be considered as a rough guideline only, and the final model selection should remain conditional on a detailed examination of the parameter estimates and theoretical conformity of the various models. Thus, before moving to a description of the B-ESEM model, we first start with a comparison of the ICM-CFA and ESEM to investigate the presence of constructrelevant psychometric multidimensionality due to the fallible nature of indicators and the presence of conceptually-related constructs. We then contrast the ESEM and B-ESEM to investigate constructrelevant psychometric multidimensionality due to hierarchically-superior constructs.

ESEM versus CFA. The ICM-CFA and ESEM solutions differ in their factor correlations (see Table 2) with much lower factor correlations for ESEM $(|r|=.006$ to $r=.648, M=.237)$ than ICM-CFA $(|r|=.106$ to $r=.815, M=.376)$. ESEM thus results in a clearer differentiation between the self-concept factors than ICM-CFA. Interestingly, simulation studies showed that ESEM tends to provide a better representation of the true correlations between factors (Asparouhov \& Muthén, 2009; Marsh, Lüdtke et al., 2013; Schmitt \& Sass, 2011), leading to the recommendation that ESEM should be retained when the estimated factor correlations are substantially reduced in comparison to ICMCFA (Marsh et al., 2009; Morin, Marsh et al., 2013). Here, the highest correlations involve either the global self-esteem factor -supporting the need for a bifactor representation - or associations between conceptually close constructs (such as peer and appearance self-concepts, or math competence and affect) - apparently supporting the theoretical adequacy of ESEM. Parameter estimates from these models are reported in the online supplements (Table S2).

An examination of the ESEM parameter estimates reveals well-defined factors due to substantial target factor loadings (varying from $|\lambda|=.014$ to $.907 ; M=.606$ ). Furthermore, the more hierarchically superior constructs (global self-esteem: target $|\lambda|=.239$ to $.668, M=.491$; general academic competence: target $|\lambda|=.014$ to $.382, M=.286$; general academic affect: target $|\lambda|==.211$ 
to $.605, M=.503$ ) tend to be less well defined than the other factors (target $|\lambda|=.350$ to $.917, M=$ .664), supporting the need for a bifactor model. Similarly, as expected, multiple non-target crossloadings are also present, providing additional support for the ESEM solution. The majority of the more substantial non-target cross-loadings (> .200) involve hierarchically-superior (global selfesteem, and general academic competence or affect) or conceptually-related constructs (e.g., peer and appearance self-concepts); and are particularly pronounced between the academic affect and competence subscales associated with the same domain. These results provide clear evidence that both sources of construct-relevant psychometric multidimensionality are present in the SDQ-I, supporting the need to rely on ESEM and suggesting the appropriateness of exploring B-ESEM.

ESEM versus B-ESEM. As previously noted, B-ESEM provides a slightly better fit to the data (according to both fit indices and lower values for the information criteria) than ESEM. The parameter estimates from this model are reported in Table 3. The B-ESEM solution shows that the GFactor is well-defined by the presence of strong and significant target loadings from most of the SDQI items $(|\lambda|=.118$ to $.691, M=.444)$, which is impressive for a G-factor defined by 76 items designed to tap into different domains. In particular, the items designed to specifically assess global self-esteem all present elevated target loadings on this G-factor $(|\lambda|=.320$ to $.610, M=.490)$. Over and above this G-factor, the S-factors related to SDQ-I subscales theoretically located at the lower level of the selfconcept hierarchy are also well-defined through substantial target loadings $(|\lambda|=.307$ to $.809, M=$ .567), suggesting that they do indeed tap into relevant specificity and add information to the selfconcept G-factor. In contrast, and supporting the appropriateness of a B-ESEM representation of the data, the items associated with most of the hierarchically superior subscales apparently present either no (general academic competence: target $|\lambda|=-.011$. to $.099, M=.066$, all non-significant at $p \leq .05$ ) or low levels (global self-esteem: target $|\lambda|=.101$ to $.411, M=.310$; general academic affect: target $|\lambda|$ $=.174$ to $.418, M=.354$ ) of meaningful residual specificity once the G-factor is taken into account. However, at least in regard to the global self-esteem and general academic affect subscales the target loadings on the S-Factors (14 out of 15 possible loadings) remain significant, supporting the need to control for this content specificity in the model, which may reflect in part the presence of additional 
self-concept domains not covered in the SDQ-I (e.g., arts, biology, spirituality; Marsh, 2007; Vispoel, 1995). This explanation is not sufficient to explain why the target loadings are so much weaker on the general academic competence S-factor than the global self-esteem and general academic affect Sfactors. A possible explanation for this difference appears related to the fact that the global academic competence items present more numerous, and stronger, cross-loadings involving domain-specific Sfactors than the items related to global self-esteem and general academic affect (also see subsequent discussion of cross-loadings). It would be possible for applied researchers to pursue a post hoc modification of this model by taking out the general academic competence S-factor and allowing global academic competence items to contribute solely to the G-factor. This alternative representation would be in line with Brunner et al. $(2008,2009,2010)$ "Nested Marsh/Shavelson model".2

Further examination of the B-ESEM solution reveals that, outside of the academic area, few items present meaningful non-target cross-loadings. Some of these cross-loadings support previous results showing partial conceptual overlap between physical appearance on the one hand and peer self-concept or physical ability on the other hand (Arens et al., 2013; Marsh, 2007; Marsh \& Ayotte, 2003). For example, some physical appearance items show substantial cross-loadings on the physical ability (e.g., Item 46: "I have a good looking body"; cross-loading = .219) or peer self-concept (e.g., Item 38: "Other kids think I am good looking"; cross-loading = .281) scales. Similarly, one peer selfconcept item also displays a substantial cross-loading on the physical appearance scale (Item 36: "I am easy to like"; cross-loading = .232). However, non-target cross-loadings appear more pronounced within the academic area. Thus, multiple items from the competence subscales present small to moderate cross-loadings on their affect counterparts, and vice-versa. For instance, items of math competence reveal cross-loadings on math affect $(|\lambda|=.230$ and $.347, M=.314)$, and items of math affect demonstrate cross-loadings on math competence $(|\lambda|=.253$ and $.298 ; M=.275)$, while the target loadings still suggest that these factors are properly defined (math competence: $|\lambda|=.557$ to $.630 ; M=$ .599 ; math affect: $|\lambda|=.644$ to $.763 ; M=.689)$. Similar results are observable for the German affect and competence subscales, as well as for the general academic competence and affect subscales, although these more general factors are not as well-defined as the domain-specific math and German 
subscales. These results confirm the distinction between competence and affect components in the academic area, but also show that the items still present a high level of specificity over and above their competence or affect nature. This explains the previously reported elevated correlations between the affect and competence subscales associated with a single domain (Arens, Yeung, Craven, \& Hasselhorn, 2011; Marsh \& Ayotte, 2003). No such pattern of non-target cross-loadings between competence and affect factors can be observed across the German and math domains supporting the strong differentiation of academic self-concept into math and verbal domains (Möller et al., 2009). Furthermore, the items forming the general academic competence and affect factors also present substantial non-target cross-loadings on their German and math counterparts, a result in line with the hierarchical nature of self-concept.

Measurement Invariance. We now turn to tests of invariance across gender of the final BESEM model (see Table 1). The model of configural invariance provides an acceptable fit to the data $(\mathrm{CFI}=.960 ; \mathrm{TLI}=.942 ; \mathrm{RMSEA}=.028)$. From this model, invariance constraints across gender were progressively added to the factor loadings (weak invariance), items' intercepts (strong invariance), items' uniqueness (strict invariance), correlated uniquenesses for parallel-worded items, latent variances and covariances, and latent means. None of these constraints resulted in a decrease in model fit exceeding the recommended cut-off scores for the fit indices ( $\triangle \mathrm{CFI}$ and $\Delta \mathrm{TLI}<.01 ; \triangle \mathrm{RMSEA}<$ .015), supporting the invariance of the B-ESEM factor structure across gender. Invariance is also generally supported by the information criteria, with the CAIC and BIC showing consistent decreases (or at least very low increases) up to the inclusion of invariance constraints on the latent variances and covariances. A more careful examination reveals a single major difference between the conclusions that would have been reached through an examination of the changes in fit indices (suggesting complete measurement invariance), and the conclusions that would have been reached through an examination of the information criteria. Indeed, the information criteria all increased when invariance constraints were imposed on the items' intercepts, thus suggesting that a solution of partial invariance of items' intercepts could be investigated; something that we illustrate in the next study. This reinforces the imprecise nature of these guidelines, the importance of anchoring decisions in multiple sources of information (Marsh et al., 2004), and the need for further simulation studies in this area. 
However, when invariance constraints are imposed to the latent means, all information criteria show increased values. This increase in the values of the information criteria, coupled with Fan and Sivo's (2009) observation that changes in goodness-of- fit indices tend to be untrustworthy indicators of latent mean invariance, suggests that latent means may not be invariant across gender. The exploration of latent means reveals that, when boys' latent means are fixed to zero for identification purposes, girls' latent means (expressed in SD units) are significantly higher than those of the boys on the German competence $(M=.408, p \leq .05)$, and German affect $(M=.243, p \leq .05)$ S-factors.

Conversely, girls' latent means are significantly lower than boys' on the physical abilities $(M=-.606$, $p \leq .01)$, and math competence $(M=-.420, p \leq .01)$ S-factors. No gender differences are apparent on the G-factor, as well as on the global self-esteem, peer, parent, appearance, academic competence and affect, and math affect S-factors. These results follow gender stereotypes and replicate those from previous studies (e.g., Marsh, 1989; Marsh \& Ayotte, 2003).

\section{Study 2: Extended Illustration Based on a Simulated Data Set}

In order to provide a simpler and more complete pedagogical example of the use of the framework presented here, we rely on a simulated data set based on a known population model. A complete set of annotated input codes used to simulate the data and to estimate all models used in this study are provided in the online supplements. Interested readers can use these inputs to simulate their own data set and try their hand at estimating a wide variety of models. The parameter estimates for the measurement part (factor loadings and items' uniquenesses) of the population model used to simulate the data are provided in Table S3 of the online supplements, and the complete population model is illustrated in Figure 2. To keep the model simpler than in Study 1, we simulated a population model including one global factor well-defined by 12 items, which also define three S-factors. These factors were specified as orthogonal in line with typical bifactor assumptions. Each S-factor is defined mainly through a total of 4 items (items X1 to X4 define mainly S-Factor S1; items Y1 to Y4 define mainly S-Factor S2; items Z1 to Z4 define mainly S-Factor S3). We simulated the data so that one of the SFactors (i.e., S-Factor S3) was more weakly defined than the other S-factors through lower target factor loadings ( .300 to .500 , versus .550 to .650 for the other S-factors). Furthermore, each item was simulated has having a very small (-.100 or .100$)$ or small (.150 or .200$)$ non-target cross-loading on 
one additional S-factor. All non-target cross-loadings were kept under the boundaries of what is typically considered negligible in EFA/ESEM applications (Marsh, Lüdtke et al., 2013).

We simulated the data using a multiple-group set up, using two groups including 800 participants each, in order to be able to illustrate tests of measurement invariance. This also allowed us to use the grouping variable as a predictive (exogenous) covariate, so as to illustrate the MIMIC approach (e.g., Jöreskog \& Goldberger, 1975; Marsh, Tracey, \& Craven, 2006; Muthén, 1989). The population model was simulated with invariant factor loadings, invariant items' uniquenesses, invariant factor variances (set to be equal to 1), and invariant relations between constructs. One item was simulated as having a non-invariant intercept (illustrated in Figure 2 as a direct effect of the grouping variable on item Y2). Latent mean differences across groups were simulated on the G-factor and the S-Factor S1. Apart from these differences, all other means and intercepts were set to be zero. We simulated one additional CFA factor (defined by items W1 to W4) as an outcome variable, specified as influenced by the grouping variable, the G-Factor, and the S-Factor S2. Thus, the full model includes an indirect effect of the grouping variable on the outcome factor as mediated by the Gfactor. This population model aimed at providing an illustration of all possible predictive relationships among the constructs. One factor (S1) was specified as being influenced by the grouping variable but having no influence on the outcome variable. One factor (S2) was specified as having an effect on the outcome but as not being influenced by the grouping variable. One factor $(\mathrm{G})$ was specified as being influenced by the grouping variable while also having an effect on the outcome variable. Finally, the last factor (S3), which is more weakly defined than the others, was specified as being unrelated to the other constructs. Using the data simulation input provided in the online supplements, it would be easy for interested readers to define their own predictive model at the population level.

For readers preferring a description of the simulated data set that is more in line with applied research, it is easy to find suitable examples. Thus, the binary grouping variable can easily reflect gender, or cultural groups. The G- and S- factors can reflect any construct that is well suited to bifactor representations. For instance, the G-Factor could reflect a global level of Attention-Deficit Hyperactivity Disorder (ADHD) while the S-factors could reflect more specific levels of Inattention, Hyperactivity, and Impulsivity going over and above global ADHD levels and be used to define 
diagnostic subtypes (e.g., Morin, Tran et al., 2013). Alternatively, the G-factor could reflect either global Intelligence or Internalizing Disorders, whereas the S-factors could define specific cognitive strengths (e.g., Verbal Comprehension, Perceptual Reasoning, Working Memory, see Gignac \& Watkins, 2013) or symptoms (e.g., Dysphoria, Suicidality, Social Anxiety, see Simms et al., 2008). Finally, the outcome variable could, for example, reflect later levels of academic achievement or attainment, life satisfaction, or psychological well-being.

\section{Analyses and Results}

Because the data was simulated to follow multivariate normality assumptions and without missing data, all analyses were conducted using Mplus 7.11 (Muthén \& Muthén, 1998-2013) maximum likelihood (ML) estimator. We start the analyses by a comparison of ICM-CFA, bifactorCFA (B-CFA), ESEM, and bifactor-ESEM (B-ESEM) representations of the underlying structure of the scores on the indicators of the main "instrument" (i.e., items X1 to X4, Y1 to Y4, and Z1 to Z4), without taking the grouping variable or the outcome into account. These models are specified as in the previous study (see Figure 1) and in line with the population model (X1 to X4 are used to define one factor, Y1 to Y4 a second factor, and Z1 to Z4 a third factor). A first-order (CFA, ESEM) model with 3 correlated factors is mathematically equivalent to a hierarchical (CFA, ESEM) model including the same 3 first-order factors used to define a single higher-order factor. Indeed, converting a 3-factor first-order model to a hierarchical model simply involves replacing three factor correlations by three higher-order factor loadings and thus results in an empirically equivalent model in terms of degrees of freedom and fit to the data (Hershberger \& Marcoulides, 2013). For this reason, we do not investigate hierarchical models, but still report annotated inputs to illustrate their estimation in the online supplements. This allows us to focus on the comparisons between ICM-CFA and ESEM, and between first-order and bifactor models, that are critical to the framework presented here.

Table 4 presents the goodness-of-fit indices and information criteria associated with the models. Both the ICM-CFA and B-CFA solutions provide an acceptable degree of fit to the data according to the CFI (.937 and .960) and TLI (.919 and .937), but not the RMSEA (RMSEA $=.109$ and .096). In contrast, both the ESEM and B-ESEM models provide an excellent fit to the data (CFI = .996 and 1.000; TLI $=.991$ and $.999 ;$ RMSEA $=.036$ and .013 ), higher values for the information 
criteria and non-overlapping RMSEA confidence intervals in comparison with the ICM-CFA/B-CFA models. Although both the ESEM and B-ESEM models provide an excellent fit to the data, the fit of the B-ESEM model is better based on an improvement in fit indices (particularly the $\triangle$ RMSEA $=$ .023), a decrease on the AIC, BIC, and ABIC, and non-overlapping RMSEA confidence intervals. This information suggests that the B-ESEM model should be retained as providing the best representation of the data. However, as mentioned previously, this final model selection should remain conditional on a detailed examination of the parameter estimates and theoretical conformity. As we are here using simulated data, theory cannot be used to help in guiding this decision (see previous study for an illustration), but knowledge of the population model confirms the adequacy of this decision. However, before interpreting the B-ESEM model, we start with a comparison of ICMCFA and ESEM to assess construct-relevant psychometric multidimensionality due to the fallible nature of the indicators. We then contrast ESEM and B-ESEM to investigate construct-relevant psychometric multidimensionality due to hierarchically-superior constructs.

ESEM versus CFA. The ICM-CFA and ESEM solutions differ in their factor correlations (see Table 5) with lower factor correlations for ESEM $(|r|=.475$ to $r=.629, M=.542)$ than ICMCFA $(|r|=.516$ to $r=.731, M=.620)$, supporting the superiority of ESEM versus ICM-CFA. Here, knowing that the population-generating model is orthogonal alerts us to the fact that these models do not provide a full representation of the construct-relevant multidimensionality present in the scale. Parameter estimates from the ICM-CFA and ESEM models are reported in the online supplements (Table S4). An examination of the ESEM parameter estimates reveals well-defined factors due to substantial target factor loadings (varying from $|\lambda|=.642$ to $.941 ; M=.810$ ). Similarly, as expected, multiple non-target cross-loadings are also present $(|\lambda|=.009$ to $.310 ; M=.100)$, providing additional support to the ESEM solution. Although it is not possible to substantively interpret the non-target cross-loadings with simulated data, these results provide clear evidence that construct-relevant psychometric multidimensionality linked to the fallible nature of the indicators simultaneously reflecting more than one construct content is likely to be present in the data, thus supporting the need to rely on ESEM. The superiority of B-ESEM in terms of fit to the data further suggests the appropriateness of investigating for the presence of a second source of construct-relevant 
multidimensionality due to the presence of hierarchically-superior constructs.

ESEM versus B-ESEM. The parameter estimates from the B-ESEM model are reported in Table 6. This B-ESEM solution shows that the G-Factor is well-defined by the presence of strong and significant target loadings from all items $(|\lambda|=.466$ to $.791, M=.664)$. Over and above this G-factor, the S-factors are also well-defined through substantial target factor loadings $(|\lambda|=.353$ to $.691, M=$ .523), suggesting that they do indeed tap into relevant specificity and add information to the G-factor - although the S-Factor S3 appears to be slightly more weakly defined (target $|\lambda|=.353$ to $.583, M=$ $.464)$ than S-factors S1 and S2 (target $|\lambda|=.477$ to $.691, M=.691)$. Further examination of the BESEM solution reveals that significant non-target cross-loadings are still present, thus supporting the value of a B-ESEM solution over a B-CFA solution. However, these non-target cross-loadings remain generally smaller $(|\lambda|=.005$ to $.176 ; M=.073)$ than those estimated in ESEM $(|\lambda|=.009$ to $.310 ; M$ $=.100$ ), showing that the bifactor operationalization allows for a more precise distribution of the various sources of construct-relevant multidimensionality present in the instrument.

The Multiple-Group Approach to Measurement Invariance. The results from the tests of measurement invariance of the final retained B-ESEM model are reported in Table 4 . The model of configural invariance provides an excellent fit to the data $(\mathrm{CFI}=.999 ; \mathrm{TLI}=.997$; $\mathrm{RMSEA}=.021)$. From this model, invariance constraints across groups were progressively added to the factor loadings (weak invariance), intercepts (strong invariance), uniquenesses (strict invariance), latent variances and covariances, and latent means. Adding invariance constraints on the factor loadings does not result in a decrease in model fit exceeding the recommended cut-off scores for the fit indices ( $\Delta$ CFI and $\Delta T L I$ $<.01 ; \triangle \mathrm{RMSEA}<.015)$, and results in lower values for the information criteria, supporting the weak invariance of the B-ESEM model. However, adding invariance constraints on the items' intercepts results in a decrease in RMSEA exceeding the recommended value $(\triangle \mathrm{RMSEA}=.018)$ and higher values on the AIC, BIC, and ABIC, suggesting that the strong invariance of the B-ESEM model may not fully hold across groups. For this reason, we explored a model of partial invariance (Byrne, Shavelson, \& Muthén, 1989). Based on the modification indices associated with the model of strong invariance and an examination of the parameter estimates associated with the model of weak 
invariance, we decided to relax the invariance constraint of item Y2 across groups, resulting in a model of partial strong invariance. When compared to the model of weak invariance, this model results in a decrease in fit that remained lower than the recommended cut-off scores for the fit indices $(\triangle \mathrm{CFI}$ and $\Delta \mathrm{TLI}<.01 ; \triangle \mathrm{RMSEA}<.015)$ and in lower values for the information criteria, supporting the adequacy of this model. When the parameters estimates from this model are examined, they show that group $2(M=.104)$ tends to present higher levels than group $1(M=-.177)$ on item Y2 to a degree that is in line with the specifications of the population-generating model (specifying a difference of .300 on item Y2). The results further support the strict invariance of the model, as well as the invariance of the latent variances and covariances $(\triangle \mathrm{CFI}$ and $\Delta \mathrm{TLI}<.01 ; \Delta \mathrm{RMSEA}<.015$; lower values for the AIC, CAIC, BIC, ABIC). However, adding invariance constraints on the latent means results in an increase on the information criteria and the highest changes in fit indices observed so far $(\Delta \mathrm{CFI}=-0.008 ; \Delta \mathrm{TLI}=-.010 ; \Delta \mathrm{RMSEA}=+.026)$. The results further show that when latent means are fixed to zero in group 1, latent means (in SD units) are significantly higher in group 2 on the GFactor $(M=.455, p \leq .01)$ but lower on the S-Factor S1 $(M=-.509, p \leq .01)$. No differences are apparent on the S-Factors S2 or S3. These results are in line with the population model (specifying opposite differences of .500 on the G-factor and S-Factor S1).

The MIMIC Approach to Measurement Invariance. The multiple-group approach to measurement invariance provides a general framework for tests of measurement invariance when the grouping variable has a small number of discrete categories and the sample size for each group is reasonable. This approach can easily be extended to tests of longitudinal measurement invariance (for a pedagogical illustration using ESEM, see Morin, Marsh et al., 2013). Nevertheless, this approach might not be practical for continuous variables (e.g., SES, IQ level, age), multiple contrast variables (e.g., gender, cultural groups, experimental/control groups) and their interactions, or small sample sizes. In such situations, a more parsimonious MIMIC approach (Jöreskog \& Goldberger, 1975; Marsh et al., 2006; Muthén, 1989) might be more appropriate. A MIMIC model is a regression model in which latent variables are regressed on observed predictors that can be extended to test potential non-invariance of item intercepts, that is, differential item functioning (DIF, monotonic DIF in the case of intercept non-invariance). Marsh, Nagengast et al. (2013) extended this approach to 
investigate the loss of information due to categorizing continuous variables (to convert them to grouping variables for more complete tests of measurement invariance) through the separate estimation of a MIMIC model in each of the groups formed by the categorization of the continuous predictors. However, while the MIMIC model is able to test monotonic DIF, it implicitly assumes the invariance of the factor loadings (non-monotonic DIF). Although MIMIC models can be extended, through the incorporation of tests of latent interactions between predictors and factor scores, to tests of non-monotonic DIF, this extension is not yet available within the ESEM or B-ESEM frameworks (Barendse, Oort, \& Garst, 2010; Barendse, Oort, Werner, Ligtvoet, \& Schermelleh-Engel, 2012).

The MIMIC model is more parsimonious than the multiple-group approach as it does not require the estimation of a separate model in each group, which makes it more suitable to smaller samples. The MIMIC approach also allows for the consideration of multiple independent variables, some or all of which can be continuous, and their interactions - something that is typically difficult to properly manage in multiple-group analyses. Monotonic DIF is evaluated by the comparison of three nested MIMIC models. In the first (null effect) model, the predictors have no effect on the latent means and items' intercepts. In the second (saturated) model, the predictors are allowed to influence all items' intercepts, but not the latent means. The third (invariant) model assumes the invariance of items' intercepts across levels of the predictors, which are allowed to influence all latent means but not items' intercepts. When the fit of the second and third models is better than the fit of the first model, the predictors can be assumed to have an effect. Comparing the second and third models tests whether the effects of the predictors on the items are fully explained by their effects on the latent means. Monotonic DIF is demonstrated when the fit of the second model is greater than the fit of the third model. Tests of partial invariance may then be pursued by including the direct effects of the predictors on the intercepts over and above their effects on the latent means.

The results from MIMIC models where the grouping variable was treated as a predictor of the latent factors are reported in Table 4. The null effects model provides an acceptable fit to the data according to commonly used interpretation guidelines (CFI and TLI $>.95$; RMSEA $<.06$ ), suggesting limited effects of the grouping variable. However, both the saturated and invariant models provide an improved level of fit to the data $(\Delta \mathrm{CFI}$ and $\Delta \mathrm{TLI}=+.008$ to $.027, \Delta \mathrm{RMSEA}=-.021$ to -.046 , and 
lower values for all of the information criteria). This suggests that the grouping variable must have an effect, at least on the latent means. When these two models are contrasted, the fit of the saturated model appears to be better than the fit of the invariant model according to the TLI $(\Delta \mathrm{TLI}=+.010)$, RMSEA ( $\triangle$ RMSEA $=-.025$ ), and the information criteria. This suggests that the effects of the grouping variable are not limited to the latent means, but also extend to some of the items' intercepts (providing evidence of monotonic DIF). Examination of the modification indices associated with the invariant model and of the parameter estimates from the saturated model suggests that DIF is mainly associated with item Y2 (which we know to be the case based on the known population values). Allowing for direct effects of the grouping variable on $\mathrm{Y} 2$ resulted in a fit to the data that was equivalent to the fit of the saturated model $(\triangle \mathrm{CFI}$ and $\triangle \mathrm{TLI}=0$, and $\triangle \mathrm{RMSEA}=-0.003)$ and in lower information criteria. Detailed results from this model reveal (in line with known population values) that participants' levels on the G-factor $\left(b=.455 ; \beta=.222, p<.001 ; \mathrm{R}^{2}=.049\right)$ and item Y2 $(b=$ $\left..278 ; \beta=.131, p<.001 ; \mathrm{R}^{2}=.740\right)$ tended to be higher in the second group, while levels on the $\mathrm{S}$ Factor $\mathrm{S} 1$ tended to be lower in the second group $\left(b=-.509 ; \beta=-.247, p<.001 ; \mathrm{R}^{2}=.061\right)$.

Predictive Models. All models considered so far can easily be extended to test predictive relationships between constructs. To illustrate tests of predictive relationships, we simulated a data set including a grouping variable specified as predicting the B-ESEM factors (i.e., an exogenous predictor), and one additional latent CFA factor specified as being predicted by the B-ESEM factors (i.e., a distal outcome). These variables thus form the predictive system illustrated in Figure 2. More precisely, the relations among these constructs were simulated according to a partially mediated predictive system such that the effect of the exogenous predictor on the distal outcome are both direct, and indirect, being mediated by the effect of the exogenous predictor on the G-factor from the set of B-ESEM factors, which in turn also predicts the distal outcome. Mediation occurs when some of the effects of an independent variable (IV; here the exogenous predictor) on the dependent variable (DV; here the distal outcome) can be explained in terms of another mediating variable (MV; here the BESEM factors) (MacKinnon, 2008). A mediator is thus an intervening variable accounting for at least part of the relation between an exogenous predictor and a distal outcome such that the exogenous predictor influences the distal outcome indirectly through the mediator(s). 
Given the objective of the current paper to illustrate a psychometric framework allowing for the analysis of sources of construct-relevant multidimensionality present in a measurement model, our main objective here is to illustrate how this psychometric framework can be used in the estimation of predictive models. However, in the interest of space, we assume that readers are reasonably familiar with tests of mediation conducted within the SEM framework and only expand on issues that are specific to the bifactor-ESEM context. Readers not familiar with mediation testing and wishing to improve their knowledge in this area may consult a number of user-friendly introductions (e.g., Hayes, 2013; Jose, 2013; MacKinnon, 2008; MacKinnon, Fairchild, \& Fritz, 2007).

Typically, tests of mediation involve contrasting two models to verify whether the mediation is complete, or whether direct effects of the exogenous predictor(s) on the distal outcome(s) remain significant over and above their effects on the mediator(s). The fit statistics associated with these two models are reported in Table 4. The fully mediated model, where the exogenous predictor is allowed to influence the B-ESEM factors (as well as item Y2 based on the results from the MIMIC model) and the B-ESEM factors are allowed to influence the distal outcome, present a satisfactory level of fit to the data $($ CFI and TLI $>.95$; RMSEA $<.06)$. However, the fit of the partially mediated model, including an additional relation between the exogenous predictor and the distal outcome, is better according to lower values for the RMSEA $(\triangle \mathrm{RMSEA}=-0.018)$ and all information criteria. The parameter estimates from this model are in line with the population-generating model and show significant effects of the exogenous predictor on the $\mathrm{G}$-factor $\left(b=.453 ; \beta=.221, p<.001 ; \mathrm{R}^{2}=.049\right)$, the S-factor $\mathrm{S} 1\left(b=-.507 ; \beta=-.246, p<.001 ; \mathrm{R}^{2}=.060\right)$, and the distal outcome $(b=.270 ; \beta=.210$, $p<.001)$, as well as significant effects of the G-factor $(b=.279 ; \beta=.445, p<.001)$ and the S-factor $\mathrm{S} 2(b=.207 ; \beta=.322, p<.001)$ on the distal outcome $\left(\mathrm{R}^{2}=.404\right)$. These results suggest that the effects of the exogenous predictor on the distal outcome are partially indirect and mediated through the effect of the exogenous predictor on the G-factor.

It is well documented that bootstrapped confidence intervals (CI) are the most efficient manner for testing the significance of indirect (mediated) effects (represented as the product of the IV $\rightarrow \mathrm{MV}$ and the MV $\rightarrow$ DV path coefficients) (see Cheung \& Lau, 2008; MacKinnon, Lockwood, \& Williams, 2004). Unfortunately, bootstrapping is not yet implemented in ESEM or B-ESEM, which represents 
another limitation of the way these models are currently implemented. However, these tests can easily be implemented using the ESEM-Within-CFA (EWC) approach described by Morin, Marsh et al. (2013; also see Marsh, Nagengast et al., 2013, as well as the input files provided here in the online supplements). To implement this method, one needs to use the start values provided as part of the final predictive ESEM or B-ESEM model, to add the constraints required for identification purposes, and to re-estimate this model while requesting bootstraped estimation (see the online supplements for details of implementation). When our final model is re-estimated using this method and requesting bias-corrected bootstrapped confidence intervals, the results confirm that the indirect effect of the exogenous predictor on the distal outcome, as mediated by the G-factor, is significant as indicated by a bias-corrected bootstrapped 95\% CI excluding 0 (indirect effect $=b=.127 ; 95 \% \mathrm{CI}=.088 / .174$ ).

Another limitation of current implementations is that all factors forming a single set of ESEM or B-ESEM factors (i.e., a set of factors is defined as a group of factors defined by the same collection of items allowed to have their main loadings or cross-loadings on all factors included in the group) are required to be simultaneously related to other variables in the same manner (Asparouhov \& Muthén, 2009; Marsh et al., 2009). Attempts to estimate a model where the factors forming a single set are specified as having a different pattern of relations to other constructs (e.g., the exogenous predictor predicts $\mathrm{S} 1$ and $\mathrm{G}$, but not $\mathrm{S} 2$ and $\mathrm{S} 3$; or including a regression between $\mathrm{S} 1$ and the outcome, but a correlation path between S2 and the outcome) would simply fail and produce a warning saying that the model has been misspecified. In the present study, this means that the exogenous predictor needed to be allowed to simultaneously predict the G-factor and the $3 \mathrm{~S}$-factors. In turn, the G-factor and the $3 \mathrm{~S}$-factors was simultaneously allowed to predict the distal outcome factor. Although this was not necessary in the present application, the EWC method could have been used to circumvent this limitation. To do so, one would simply need to use the start values from the final ESEM or B-ESEM measurement model, to add the constraints required for identification purposes, and to replace the factor correlations linking the ESEM/B-ESEM factors to the other variables by the required predictive paths. We provide a sample input in the online supplements illustrating the implementation of this method to estimate only the predictive paths depicted in Figure 2.

\section{General Discussion}




\section{An Integrative Framework to Investigate Source of Construct-Relevant Multidimensionality}

This study illustrated an overarching psychometric approach of broad relevance to investigations of many complex multidimensional instruments routinely used in psychological and educational research. More precisely, we showed how an integration of classical (CFA), emerging (ESEM) and "rediscovered" (bifactor) models provides a general framework (bifactor-ESEM) for the investigation of two sources construct-relevant psychometric multidimensionality related to (a) the hierarchical nature of the constructs being assessed (i.e., the co-existence of global and specific components within the same measurement model) and (b) the fallible nature of indicators which tend to include at least some degree of association with non-target constructs. We argue that the first source of construct-relevant multidimensionality naturally calls for bifactor models, while the second source of construct-relevant multidimensionality rather calls for ESEM (rather than CFA). Thus, when both sources of multidimensionality are present, then a bifactor-ESEM model should be preferable. Such integrated models have only recently be made available and had yet to be systematically applied to the investigation of complex measurement instruments.

The first step in the application of the proposed framework starts with a comparison of firstorder ICM-CFA and ESEM models to assess the presence of construct-relevant multidimensionality due to the fallible nature of the indicators and reinforced by the presence of conceptually-related or overlapping constructs. Given that bifactor models tend to absorb un-modeled cross-loadings through the estimation of inflated global factors (Murray \& Johnson, 2013), it is important that the application of this framework always starts with a comparison of ESEM versus ICM-CFA models. In agreement with previous recommendations (e.g., Marsh et al., 2014; Morin, Marsh et al., 2013), we argue that this first comparison should routinely be conducted in the investigation of the measurement structure of any multidimensional instrument. In the examples provided in this manuscript, ESEM solutions provided a better fit to the data when compared to ICM-CFA models. The superiority of ESEM was further corroborated by the observation of lower factor correlations, resulting in more clearly differentiated factors. In line with previous recommendations (e.g., Morin, Marsh et al., 2013), applied studies (e.g., Marsh et al., 2009, Marsh, Lüdtke et al., 2010; Morin \& Maïano, 2011), and simulations (Asparouhov \& Muthén, 2009; Marsh, Lüdtke et al., 2013; Schmitt \& Sass, 2011), these 
observations converge in supporting the superiority of the ESEM solution - at least in the data sets considered here. As previously reinforced, decisions regarding the appropriateness of alternative models to represent sources of construct-relevant multidimensionality should not be taken in disconnection from a detailed examination of parameter estimates and substantive theory. The ESEM results from Study 1 showed that all factors were clearly defined by the expected pattern of target loadings and non-target cross-loadings, with stronger cross-loadings between conceptually-related or hierarchically-ordered scales. This last observation suggested the presence of construct-relevant multidimensionality involving hierarchically-superior constructs.

The second step in the application of the proposed framework involves the comparison of first-order versus bifactor and higher-order solutions (relying on ESEM or CFA depending on the results from the first step), in order to assess the presence of construct-relevant multidimensionality due to the presence of hierarchically-superior constructs. Although we argued that the first step of this framework should be routinely applied to the investigation of any multidimensional instrument, this second verification should only be conducted when substantive theory and the results from the first step suggest that this second source of construct-relevant multidimensionality may be present in an instrument. In the examples considered here, the bifactor-ESEM was retained as providing the best fitting representation of the data after verification of theoretical (in Study 1) and empirical (in both studies) conformity of the parameter estimates. Indeed, in the bifactor-ESEM solutions, the G-factors were well-defined and clearly supported the presence of a global factor emerging from answers to the full set of items. It is true that, in Study 1, the inclusion of items specifically designed to assess global self-conceptions made the SDQ-I uniquely well suited to this illustration. However, the application of bifactor models is in no way dependent on the presence of items directly tapping into a global construct (e.g., Gignac \& Watkins, 2013; Morin, Tran et al., 2013; Simms et al., 2008).

\section{The Meaning of the Alternative Models}

\section{Construct-Relevant Multidimensionality: Items as Fallible Indicators of a Single}

Construct. A common idea in applied research is that good indicators need to provide a perfect reflection of a single construct, and that cross-loadings will inherently and irremediably change the meaning of the constructs that are estimated. Rather, following Marsh et al. (2014), we argue here that 
a completely pure item that has no cross-loadings or other sources of non-random specificity is a convenient fiction - at best an impossible ideal and at worst a potentially serious distortion of reality that undermines the valid interpretation of the data. Seeking such ideals, absolute truths, and other golden rules in psychometrics that obviate subjective interpretations (Marsh et al., 2004) is not inherently bad. However, applied researchers need to understand that pure items do not exist in reality and will be rejected in a purely statistical sense when evaluated within a sufficiently broad framework (with large Ns and a sufficiently large number of items and factors). Of course, misfit associated with cross-loadings might be sufficiently trivial to be ignorable - providing an appropriate balance between complexity and parsimony - but support for such claims should be based on empirical results.

The simple observation that many items are inherently expected to include construct-relevant multidimensionality explaining their association with multiple constructs shows that this requirement for pure indicators relies on a logic that inherently flawed. For example, in the assessment of anxiety and depression (e.g., Gignac et al., 2007; Simms et al., 2008), cross-loadings are expected due to the fact that some symptoms are inherently part of both disorders, such as insomnia and psychomotor agitation. Our illustration based on the SDQ-I also provides interesting examples. For instance, "I have a good looking body" (an indicator of physical appearance self-concept) had a significant nontarget cross-loading on physical ability self-concept, which could be related to the fact that athletic bodies tend to be perceived as more attractive. Similar examples are numerous and show that crossloadings do not "taint" the constructs, but rather allow the constructs to be estimated using all of the relevant information present at the indicator level. Remember that, according to the reflective logic of factor analyses, the factors are specified as influencing the indicators, rather than the reverse. Thus, small cross-loadings should be seen as reflecting the influence of the factor on the construct-relevant part of the indicators, rather than the indicators having an impact on the nature of the factor itself. It should be kept in mind that this interpretation applies to relatively small cross-loadings that are in line with theoretical expectations, whereas any model showing large and unexplainable cross-loadings or cross-loadings larger than target loadings should be re-examined.

Furthermore, factor correlations tend to be substantially biased when non-zero cross-loadings are constrained to be zero (as shown in our simulated data set, as well as in Asparouhov \& Muthén, 
2009; Marsh, Lüdtke et al., 2013; Schmitt \& Sass, 2011). This suggests that it is the exclusion of these cross-loadings that can drastically change the meaning of the constructs. This clearly underlines the importance for applied research to consider this additional source of construct-relevant psychometric multidimensionality even when the initial ICM-CFA model appears to fit the data well (Marsh, Liem et al., 2011; Marsh, Nagengast et al., 2011). As noted by Marsh et al. (2014, p. 104): "If the fit and parameter estimates (e.g., latent factor correlations) for the ICM-CFA do not differ substantially from the corresponding ESEM, on the basis of parsimony researchers should retain the CFA model". Alternatively, when the fit of the ESEM solution is acceptable, higher than the fit of the ICM-CFA, and ESEM results in lower estimates of the factor correlations, then ESEM should be preferred.

It is true that rotational indeterminacy raises additional questions. Indeed, any ESEM solution depends on the rotation procedure that is selected so that factors correlations and non-target crossloading can be directly increased or decreased by changing the rotation (Morin, \& Maïano, 2011; Schmitt \& Sass, 2011). With this in mind, simulation studies still show that, notwithstanding this issue, ESEM tends to provide factor correlation estimates that are closer to true population values, even if they are themselves imperfect due to rotational indeterminacy (e.g., Asparouhov \& Muthén, 2009; Marsh, Lüdtke et al., 2013; Schmitt \& Sass, 2011). Furthermore, even when the true population model corresponds to ICM-CFA, ESEM still tends to adequately recover true population values. In this paper, we elected to rely on target rotation, which provides a confirmatory approach to ESEM (see Marsh et al., 2014) and allows the analyst to specify the expected pattern of associations between items and factors. Furthermore, when bifactor-ESEM models are specified as orthogonal (e.g., Chen et al., 2006; Reise, 2012), these concerns are somehow diminished. However, the reader should keep in mind that even for bifactor-ESEM, the selection of an orthogonal (versus oblique) rotation is itself a choice and subject to rotational indeterminacy (see Endnote 1 and Jenrich \& Bentler, 2012).

Construct-Relevant Multidimensionality: Hierarchically-Superior Constructs. Although our main focus is on bifactor models as a method of choice to model construct-relevant multidimensionality due to the presence of hierarchically-superior constructs, we also contrasted these models with hierarchical models in t study 1 . The SLP (described earlier) makes obvious that both models estimate some form of global factor based on the covariance shared among all items, as well 
as variance components reflecting specificity associated with groupings of items that remain unexplained by the global factor (see also Chen et al., 2006). However, there are critical differences between the two approaches. Statistically, the strict proportionality constraints that are at play in hierarchical models limit their flexibility and practical applicability (Brunner et al., 2012; Chen et al., 2006; Jenrich \& Bentler, 2011; Reise, 2012). In line with this affirmation, the hierarchical-CFA and hierarchical-ESEM systematically provided the worst fit to the data of all models estimated in the first study. Substantively, the difference between these models is even more pronounced. A bifactor model assumes the existence of an overarching construct underlying all indicators, and estimates the Sfactors from the part of the indicators that remains unexplained by this global component. The Sfactors are thus seen as conceptually distinct from the G-factor. Conversely, a hierarchical model directly estimates the global factor from the first-order factors, rather than the indicators. The firstorder factors are thus a component of the global factor, rather than being separate from it.

As shown in our illustration based on simulated data, even when the true underlying population model follows bifactor assumptions, it is possible for alternative first-order CFA or ESEM models to provide a satisfactory level of fit to the data through the simple "absorption" of this hierarchical structure via inflated factor correlations and/or item cross-loadings. However, these alternative models are substantively erroneous in that they completely ignore the underlying global construct that underlies responses to all indicators. In psychiatric measurement, Morin, Tran et al. (2013, p. 2) note that "an important question has to do with whether a primary dimension (e.g., depression, anxiety, ADHD, etc.) does exist as a unitary disorder, including specificities (i.e., as represented by a bifactor model), or whether these specificities rather define a set of distinct facets without a generic common core (i.e., represented by a classical CFA model)". Fortunately, a detailed assessment of parameter estimates, theory, and statistical indices allowed us to pick the proper model in this simulated data study, although the fit of the alternative models generally proved satisfactory according to typical interpretation guidelines. Clearly, future simulation studies should investigate more thoroughly the efficacy of the various goodness-of-fit indices and information criteria in selecting the proper model amongst the alternative representations considered here.

\section{Conclusion}


This study was designed to illustrate an overarching psychometric framework for the investigation of construct-relevant multidimensionality related to the fallible nature of the imperfect indicators typically used in applied research, and to the assessment of hierarchically-superior constructs within the same instrument. Although our results supported the use of bifactor-ESEM, we do not claim that this specific psychometric representation will necessarily generalize to all instruments that are routinely used in psychological, educational, and social sciences research. However, we anticipate that this specific combination (i.e., bifactor-ESEM) may prove to be quite important to consider when working with complex multidimensional measures. More generally, we argue that the full framework proposed here should be routinely applied to studies of complex instruments, especially those that include a separate subset of items specifically designed to assess hierarchically-superior constructs. In these contexts, we believe that typical solutions of modeling these items as a separate subscale, or simply of excluding them, should no longer be seen as adequate as it ignores the inherently hierarchical nature of the assessed constructs.

\section{Endnotes}

${ }^{1}$ Bifactor estimation relies on orthogonal factors to ensure interpretability of the results. However, alternative models may be estimated where the S-factors are allowed to correlate to one another, although these models often pose interpretation and/or convergence problems. In B-CFA (or CFA more generally), orthogonal models are more parsimonious than comparative models based on oblique factors, and thus provide a different fit to the data. However, in ESEM or B-ESEM, oblique or orthogonal rotations have equivalent covariance implications and thus are statistically equivalent models with identical fit to the data. To ensure comparability with typical bifactor applications, as well as between B-CFA and B-ESEM, we relied on orthogonal rotation. However, exploring alternative procedures confirmed that our main conclusions were unaffected by this choice.

${ }^{2}$ An ESEM model including $f$ factors is empirically impossible to distinguish from a B-ESEM model including $f-1$ S-factors: Both are equivalent, have the same degrees of freedom, and produce the same chi-square, fit indices, and information criteria (Hershberger \& Marcoulides, 2013; MacCallum, Wegener, Uchino, \& Fabrigar, 1993). The reason for this is that in ESEM, each item is allowed to load on all factors. So, in a B-ESEM model including $f$ S-factors and one G-factor, each item is in fact 
allowed to load on $f+l$ factors. This makes an ESEM model including $f$ factors impossible to distinguish from a B-ESEM model including $f-1$ S-factors as both, in the end, will estimate the loadings of all items on a total of $f$ factors. In fact, differences in results between these two solutions can be attributed to the inherent rotational indeterminacy of any EFA/ESEM application (e.g., Morin \& Maïano, 2011; Morin, Marsh et al., 2013). For this reason, we recommend starting all comparisons by contrasting an ESEM model including $f$ factors with a B-ESEM model including the same number of S-factors (i.e., only differing by the addition of the G-factor). Whenever the results from both models provide an adequate and similar level of fit to the data, then the results from the B-ESEM model should be systematically inspected in order to verify whether it makes sense to drop one of the S-factor including items that should theoretically relate only to the G-factor.

\section{References}

Abu-Hilal, M.M., \& Aal-Hussain, A.Q.A. (1997). Dimensionality and hierarchy of the SDQ in a nonwestern milieu: A test of self-concept invariance across gender. Journal of Cross-Cultural Psychology, 28, 535-553.

Akaike, H. (1987). Factor analysis and AIC. Psychometrika, 52, 317-332.

Arens, A.K., Yeung, A.S., Craven, R.G., \& Hasselhorn, M. (2011). The twofold multidimensionality of academic self-concept: Domain specificity and separation between competence and affect components. Journal of Educational Psychology, 103, 970-981.

Arens, A.K., Yeung, A.S., Craven, R.G., \& Hasselhorn, M. (2013). A short German version of the Self-Description Questionnaire I: Theoretical and empirical comparability. International Journal of Research \& Method in Education, 36, 415-438.

Asparouhov, T. \& Muthén, B.O. (2009). Exploratory structural equation modeling. Structural Equation Modeling, 16, 397-438.

Barendse, M.T., Oort, F.J., \& Garst, G.J. (2010). Using restricted factor analysis with latent moderated structures to detect uniform and nonuniform measurement bias: A simulation study. AStA-Advances in Statistical Analysis, 94, 117-127.

Barendse, M.T., Oort, F.J., Werner, C.S., Ligtvoet, R., \& Schermelleh-Engel, K. (2012). Measurement bias detection through factor analysis. Structural Equation Modeling, 19, 561-579. Bentler, P. (1990). Comparative fit in structural models. Psychological Bulletin, 107, 238-246. 
Bollen, K.A. (1989). Structural Equations with latent variables. New York, NY: Wiley.

Bozdogan, H. (1987). Model Selection and Akaike's information criterion (AIC): The general theory and its analytical extensions. Psychometrika, 52, 345-370.

Browne, M.W. (2001). An overview of analytic rotation in exploratory factor analysis. Multivariate Behavioral Research, 36, 111-150

Browne, M.W., \& Cudeck, R. (1993). Alternative ways of assessing model fit. In K. A. Bollen, \& J. S. Long (Eds.), Testing structural equation models (pp. 136-162). Newbury Park, CA: Sage.

Brunner, M., Keller, U., Dierendonck, C., Reichert, M., Ugen, S., Fischbach, A., \& Martin, R. (2010). The structure of academic self-concepts revisited: The nested Marsh/Shavelson model. Journal of Educational Psychology, 102, 964-981.

Brunner, M., Keller, U., Hornung, C., Reichert, M. \& Martin, R. (2009). The cross-cultural generalizability of a new structural model of academic self-concepts. Learning and Individual Differences, 19, 387-403.

Brunner, M., Lüdtke, O., \& Trautwein, U. (2008). The internal/external frame of reference model revisited: Incorporating general cognitive ability and general academic self-concept. Multivariate Behavioral Research, 43, 137-172.

Brunner, M., Nagy, G., \& Wilhelm, O. (2012). A tutorial on hierarchically structured constructs. Journal of Personality, 80, 796-846.

Byrne, B.M. (1996). Measuring self-concept across the life span: Issues and instrumentation. Washington, DC: American Psychological Association.

Byrne, B.M., Shavelson, R.J., \& Muthén, B.O. (1989). Testing for the equivalence of factor structures: The issue of partial measurement invariance. Psychological Bulletin, 105, 456-466.

Caci, H., Morin, A.J.S., \& Tran, A. (2013, in press). Teacher ratings of the ADHD-RS IV in a community sample: Results from the ChiP-ARD study. Journal of Attention Disorders. Early view, DOI: $10.1177 / 1087054712473834$.

Chen, F.F. (2007). Sensitivity of goodness of fit indices to lack of measurement invariance. Structural Equation Modeling, 14, 464-504.

Chen, F.F., West, S.G., \& Sousa, K.H. (2006). A comparison of bifactor and second-order models of quality of life. Multivariate Behavioral Research, 41, 189-255.

Cheung, G.W., \& Lau, R.S. (2008). Testing mediation and suppression effects of latent variables: 
Bootstrapping with structural equation models. Organizational Research Methods, 11, 296-325.

Cheung, G.W., \& Lau, R.S. (2012). A direct comparison approach for testing measurement invariance. Organizational Research Methods, 15, 167-198.

Cheung, G.W. \& Rensvold, R.B. (2002). Evaluating goodness-of-fit indexes for testing measurement invariance. Structural Equation Modeling, 9, 233-255.

Eid, M., Nussbeck, F.W., Geiser, C., Cole, D.A., Gollwitzer, M., \& Lischetzke, M (2008). Structural equation modelling of multitrait-multimethod data: Different models for different types of methods. Psychological Methods, 13, 230-253.

Enders, C.K. (2010). Applied missing data analysis. New York: Guilford.

Fan, X., \& Sivo, S.A. (2005). Sensitivity of fit indexes to misspecified model components: Rationale of two-index strategy revisited. Structural Equation Modeling, 12, 343-367.

Fan, X., \& Sivo, S.A. (2007). Sensitivity of fit indices to model misspecification and model types. Multivariate Behavioral Research, 42, 509-529

Fan, X., \& Sivo, S.A. (2009). Using goodness-of-fit indexes in assessing mean structure invariance. Structural Equation Modeling, 16, 54-69.

Gignac, G.E. (2007). Multi-factor modeling in individual differences research: Some recommendations and suggestions. Personality and Individual Differences, 42, 37-48.

Gignac, G.E., Palmer, B., \& Stough, C. (2007). A confirmatory factor analytic investigation of the TAS-20: Corroboration of a five-factor model and suggestions for improvement. Journal of Personality Assessment, 89, 247-257.

Gignac, G.E., \& Watkins, M.W. (2013). Bifactor modeling and the estimation of model-based reliability in the WAIS-IV. Multivariate Behavioral Research, 48, 639-662.

Graham, J.W. (2009). Missing data analysis: Making it work in the real world. Annual Review of Psychology, 60, 549-576.

Grimm, K. J., Steele, J. S., Ram, N., \& Nesselroade, J. R. (2013). Exploratory latent growth models in the structural equation modeling framework. Structural Equation Modeling, 20, 568-591.

Hayes, A. F. (2013). Introduction to mediation, moderation, and conditional process analysis: A regression-based approach. New York: Guilford.

Hershberger, S.L., \& Marcoulides, G.A. (2013). The problem of equivalent structural models. In Hancock, G.R., \& Mueller, R.O. (Eds.). Structural equation modeling: A second course (2nd ed., 
pp. 13-42). Charlotte, NC: Information Age Publishing, Inc.

Hu, L.-T., \& Bentler, P.M. (1998). Fit indices in covariance structure modeling: Sensitivity to underparameterized model misspecification. Psychological Methods, 3, 424-453.

Holzinger, K.J., \& Swineford, F. (1937). The bi-factor model. Psychometrika, 2, 1-17.

Jennrich, R.I., \& Bentler, P.M. (2011). Exploratory bi-factor analysis. Psychometrika, 76, 537- 549.

Jennrich, R.I., \& Bentler, P.M. (2012). Exploratory bi-factor analysis: The oblique case. Psychometrika, 77, 442-454.

Jöreskog, K.G. (1973). A general method for estimating a linear structural equation system. In A.S. Golberger, \& O.D. Duncan (Eds.), Structural equation models in the social sciences (pp. 85-112). New York, NY: Seminar.

Jöreskog, K.G., \& Goldberger, A. (1975). Estimation of a model with multiple indicators and multiple causes of a single latent variable. Journal of the American Statistical Association, 10, 631-639. Jose, P.E. (2013). Doing statistical mediation and moderation. New York: Guilford.

MacCallum, R.C., Wegener, D.T., Uchino, B.N., \& Fabrigar, L.R. (1993). The problem of equivalent models in applications of covariance structure analysis. Psychological Bulletin, 114, 185-199.

MacKinnon, D.P. (2008). Introduction to statistical mediation analysis. Mahwah, NJ: Erlbaum. MacKinnon, D.P., Fairchild, A.J., \& Fritz, M.S. (2007). Mediation analysis. Annual Review of Psychology, 58, 593 -614.

MacKinnon, D.P., Lockwood, C.M., \& Williams, J. (2004). Confidence limits for the indirect effect: Distribution of product and resampling methods. Multivariate Behavioral Research, 39, 99-128. Marsh, H.W. (1987). The hierarchical structure of self-concept and the application of hierarchical confirmatory factor analyses. Journal of Educational Measurement, 24, 17-39.

Marsh, H.W. (1989). Age and sex effects in multiple dimensions of self-concept: Preadolescence to early adulthood. Journal of Educational Psychology, 82, 417-430.

Marsh, H.W. (1990). Self-Description Questionnaire - I (SDQ-I). Manual. Macarthur, N.S.W. Australia: University of Western Sydney.

Marsh, H.W. (2007). Self-concept theory, measurement and research into practice: The role of selfconcept in educational psychology. Leicester, UK: British Psychological Society.

Marsh, H.W., Abduljabbar, A.S., Abu-Hilal, M., Morin, A.J.S., Abdelfattah, F., Leung, K.C., Xu, M.K., Nagengast, B., \& Parker, P. (2013). Factor structure, discriminant and convergent validity 
of TIMSS math and science motivation measures: A comparison of USA and Saudi Arabia. Journal of Educational Psychology, 105, 108-128.

Marsh, H.W., \& Ayotte, V. (2003). Do multiple dimensions of self-concept become more differentiated with age? The differential distinctiveness hypothesis. Journal of Educational Psychology, 95, 687-706.

Marsh, H.W., Hau, K.-T., \& Grayson, D. (2005). Goodness of fit evaluation in structural equation modeling. In A. Maydeu-Olivares \& J. McArdle (Eds.), Contemporary psychometrics. A Festschrift for Roderick P. McDonald. Mahwah NJ: Erlbaum.

Marsh, H.W., Hau, K.-T., \& Wen, Z. (2004). In search of golden rules: Comment on hypothesistesting approaches to cutoff values for fit indexes and dangers in overgeneralizing Hu \& Bentler's (1999). Structural Equation Modeling, 11, 320-341.

Marsh, H.W., \& Hocevar, D. (1985). Application of confirmatory factor analysis to the study of selfconcept: First- and higher order factor models and their invariance across grades. Psychological Bulletin, 97, 562-582.

Marsh, H.W., Liem, G.A.D., Martin, A.J., Morin, A.J.S., \& Nagengast, B. (2011). Methodological measurement fruitfulness of exploratory structural equation model: New approaches to issues in motivation and engagement. Journal of Psychoeducational Assessment, 29, 322-346.

Marsh, H.W., Lüdtke, O., Muthén, B., Asparouhov, T., Morin, A. J. S., Trautwein, U., \& Nagengast, B. (2010). A new look at the big-five factor structure through exploratory structural equation modeling. Psychological Assessment, 22, 471-491.

Marsh, H.W., Lüdtke, O., Nagengast, B., Morin, A.J.S., \& Von Davier, M. (2013). Why item parcels are (almost) never appropriate: Two wrongs do not make a right—Camouflaging misspecification with item parcels in CFA models. Psychological Methods, 18, 257-284.

Marsh, H.W., Morin, A.J.S., Parker, P.D., \& Kaur, G. (2014). Exploratory structural equation modelling: An integration of the best features of exploratory and confirmatory factor analyses. Annual Review of Clinical Psychology, 10, 85-110.

Marsh, H.W., Muthén, B., Asparouhov, A., Lüdtke, O., Robitzsch, A., Morin, A.J.S., \& Trautwein, U. (2009). Exploratory structural equation modeling, integrating CFA and EFA: Application to students' evaluations of university teaching. Structural Equation Modeling, 16, 439-476.

Marsh, H.W., Nagengast, B., \& Morin, A.J.S. (2013). Measurement invariance of big-five factors 
over the life span: ESEM tests of gender, age, plasticity, maturity, and La Dolce Vita effects. Developmental Psychology, 49, 1194-1218.

Marsh, H.W., Nagengast, B., Morin, A.J.S., Parada, R.H., Craven, R.G., \& Hamilton, L.R. (2011). Construct validity of the multidimensional structure of bullying and victimization: An application of exploratory structural equation modeling. Journal of Educational Psychology, 103, 701-732.

Marsh, H.W., Scalas, L.F., \& Nagengast, B. (2010). Longitudinal tests of competing G-factor structures for the Rosenberg self-esteem scale: Traits, ephemeral artifacts, and stable response styles. Psychological Assessment, 22, 366-381.

Marsh, H.W., Tracey, D.K., \& Craven, R.G. (2006). Multidimensional self-concept structure for preadolescents with mild intellectual disabilities: A hybrid multigroup-mimic approach. Educational and Psychological Measurement, 66, 795-818.

McCrae, R.R., Zonderman, A.B., Costa, P.T.Jr., Bond, M.H., \& Paunonen, S. (1996). Evaluating the replicability of factors in the revised NEO Personality Inventory: Confirmatory factor analysis versus Procrustes rotation. Journal of Personality and Social Psychology, 70, 552-566.

Meleddu, M., Guicciardi, M., Scalas, L.F., \& Fadda, D. (2012). Validation of an Italian version of the Oxford Happiness Inventory in adolescence. Journal of Personality Assessment, 94, 175-185.

Meredith, W. (1993). Measurement invariance, factor analysis and factorial invariance. Psychometrika, 58, 525-543.

Millsap, R.E. (2011). Statistical approaches to measurement invariance. New York: Routledge. Möller, J., Pohlmann, B., Köller, O., \& Marsh, H. W. (2009). Meta-analytic path analysis of the internal/external frame of reference model of academic achievement and academic self-concept. Review of Educational Research, 79, 1129-1167.

Morin, A. J. S., \& Maïano, C. (2011). Cross-validation of the short form of the physical self-inventory (PSI-S) using exploratory structural equation modeling (ESEM). Psychology of Sport \& Exercise, $12,540-554$.

Morin, A. J. S., Marsh, H. W., \& Nagengast, B. (2013). Exploratory structural equation modeling. In Hancock, G. R., \& Mueller, R. O. (Eds.). Structural equation modeling: A second course (2nd ed., pp. 395-436). Charlotte, NC: Information Age Publishing, Inc.

Morin, A.J.S., Tran, A., \& Caci, H. (2013, in press). Factorial validity of the ADHD Adult Symptom Rating Scale in a French community sample. Journal of Attention Disorders. Early view, DOI: 


\section{$10.1177 / 1087054713488825$}

Murray, A.L., \& Johnson, W. (2013). The limitations of model fit in comparing the bi-factor versus higher-order models of human cognitive ability structure. Intelligence, 41, 407-422.

Muthén, B.O. (1989). Latent variables in heterogenous populations. Psychometrika, 54, 557-585.

Muthén, L.K., \& Muthén, B.O. (1998 -2012). Mplus user's guide. Seventh edition. Los Angeles, CA: Muthén \& Muthén.

Reise, S.P. (2012). The rediscovery of bifactor measurement models. Multivariate Behavioral Research, 47, 667-696.

Reise, S.P., Moore, T.M., \& Haviland, M.G. (2010). Bifactor models and rotations: Exploring the extent to which multidimensional data yield univocal scale scores. Journal of Personality Assessment, 92, 544-559.

Reise, S.P., Moore, T.M., \& Maydeu-Olivares, A. (2011). Targeted bifactor rotations and assessing the impact of model violations on the parameters of unidimensional and bifactor models. Educational and Psychological Measurement, 71, 684-711.

Reise, S.P., Morizot, J., \& Hays, R.D. (2007). The role of the bifactor model in resolving dimensionality issues in health outcomes measures. Quality of Life Research, 16, 19-31.

Rindskopf, D., \& Rose, T. (1988). Some theory and applications of confirmatory second-order factor analyses. Multivariate Behavioral Research, 23, 51-67.

Schmid, J., \& Leiman, J.M. (1957). The development of hierarchical factor solutions. Psychometrika, $22,53-61$.

Schmitt, T.A., \& Sass, D.A. (2011). Rotation criteria and hypothesis testing for exploratory factor analysis: implications for factor pattern loadings and interfactor correlations. Educational \& Psychological Measurement, 71, 95-113.

Schwartz, G. (1978). Estimating the dimension of a model. The Annals of Statistics, 6, 461-464.

Schweizer, K. (2012). On correlated errors. European Journal of Psychological Assessment, 28, 1-2.

Sclove, L. (1987). Application of model-selection criteria to some problems in multivariate analysis. Psychometrika, 52, 333-343.

Shavelson, R.J., Hubner, J.J. \& Stanton, G.C. (1976). Self-concept: Validation of construct interpretations. Journal of Educational Research, 46, 407-441.

Simms, L.J., Grös, D.F., Watson, D., \& O’Hara, M. (2008). Parsing general and specific components 
of depression and anxiety with bifactor modeling. Depression and Anxiety, 25, 34-46.

Steiger, J. H. (1990). Structural model evaluation and modification: An interval estimation approach. Multivariate Behavioral Research, 25, 173-180.

Tucker, L. R., \& Lewis, C. (1973). A reliability coefficient for maximum likelihood factor analysis. Psychometrika, 38, 1-10.

Vispoel, W.P. (1995). Self-concept in artistic domains: An extension of the Shavelson, Hubner, and Stanton (1976) model. Journal of Educational Psychology, 87, 134-153

Watkins, D., \& Akande, A. (1992). Internal structure of the Self-Description Questionnaire: A Nigerian investigation. British Journal of Educational Psychology, 62, 120-125.

Watkins, D., \& Dong, Q. (1994). Assessing the self-esteem of Chinese school children. Educational Psychology, 14, 129-137.

Watkins, D., Juhasz, A.M., Walker, A., \& Janvlaitiene, N. (1995). The Self-Description Questionnaire-1: Lithuanian. European Journal of Psychological Assessment, 11, 41-51.

Yung, Y.F., Thissen, D., \& McLeod, L.D. (1999). On the relationship between the higher-order factor model and the hierarchical factor model. Psychometrika, 64, 113-128. 

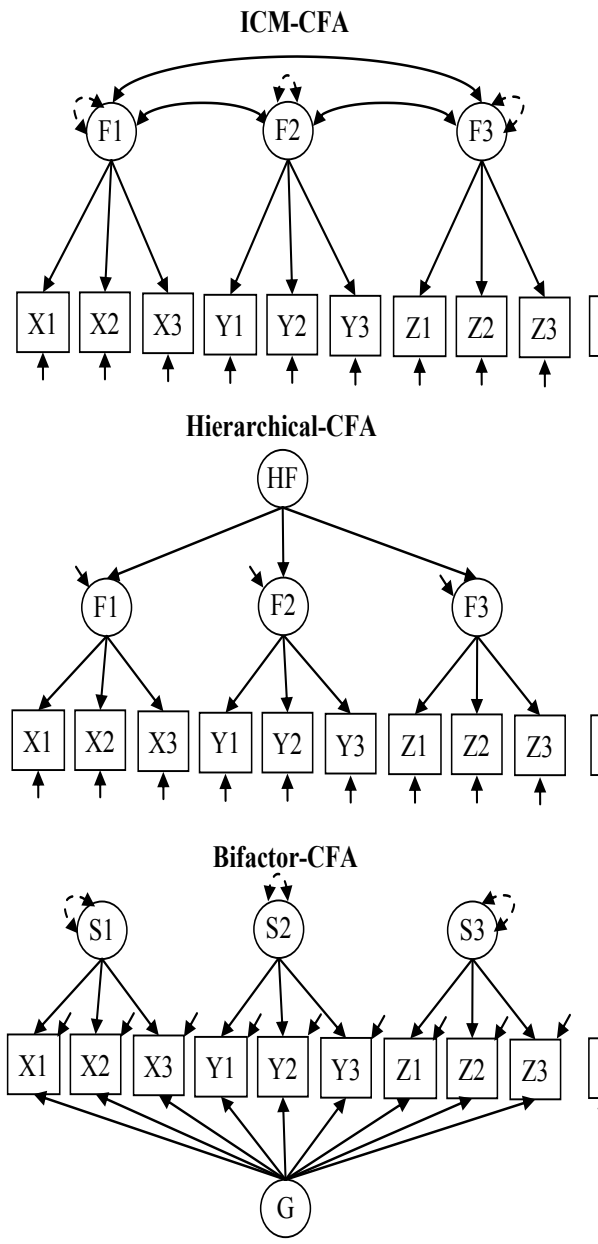

Figure 1. Graphical representation of the alternative models considered in this study.

Note. $\mathrm{CFA}=$ Confirmatory factor analyses; ICM = Independent cluster model; ESEM = Exploratory structural equation modeling; X1-X3, Y1-Y3, Z1-Z3 = Items; F1-F3: Factors; HF: Higher-order factors; S1-S3: Specific factors in a bifactor model; $\mathrm{G}=$ Global factor in a bifactor model; Ovals represent latent factors and squares represent observed variables; full unidirectional arrows linking ovals and squares represent the main factor loadings; dotted unidirectional arrows linking ovals and squares represent the cross-loadings; full unidirectional arrows linked to the items or the factors represent the item uniquenesses or factor disturbances; bidirectional full arrows linking the ovals represent factor covariances/correlations; bidirectional dashed arrows connecting a single oval represent factor variances.

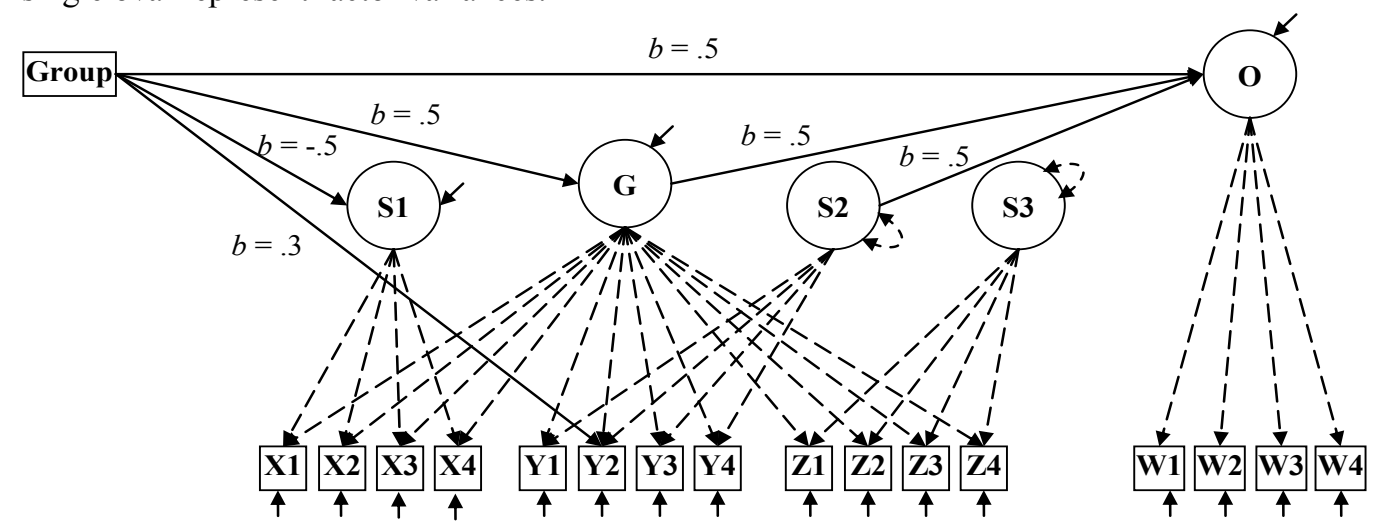

Figure 2. Graphical representation of the population generating model.

Note. Circles represent latent factors and squares represent observed variables; dotted unidirectional arrows linking ovals and squares represent the factor loadings and cross loadings; full unidirectional arrows the ovals and squares represent regressions; full unidirectional arrows placed under the squares represent the item uniquenesses; full unidirectional arrows placed under the circles the factor disturbances; bidirectional dashed arrows connecting a single oval represent factor variances. 
Table 1. Goodness of Fit Statistics and Information Criteria for the Models Estimated on the SDQ-I

\begin{tabular}{|c|c|c|c|c|c|c|c|c|c|c|}
\hline Model & $\chi^{2}$ & df & CFI & TLI & RMSEA & RMSEA 90\% CI & AIC & CAIC & $\mathrm{BIC}$ & $\mathrm{ABIC}$ \\
\hline ICM-CFA & $8417.256^{*}$ & 2677 & 0.921 & 0.916 & 0.033 & {$[0.032-0.034]$} & 362516 & 364655 & 364330 & 363297 \\
\hline $\mathrm{H}-\mathrm{CFA}$ & $12888.973 *$ & 2721 & 0.861 & 0.854 & 0.044 & {$[0.043-0.044]$} & 367961 & 369810 & 369529 & 368637 \\
\hline B-CFA & $12162.070 *$ & 2656 & 0.870 & 0.860 & 0.043 & {$[0.042-0.044]$} & 367183 & 369460 & 369114 & 368014 \\
\hline ESEM & $4760.960 *$ & 2027 & 0.963 & 0.947 & 0.026 & {$[0.025-0.027]$} & 359081 & 365496 & 364521 & 361424 \\
\hline H-ESEM & $5804.065^{*}$ & 2071 & 0.949 & 0.930 & 0.030 & {$[0.029-0.031]$} & 360295 & 366421 & 365490 & 362532 \\
\hline B-ESEM & $4183.547 *$ & 1962 & 0.970 & 0.956 & 0.024 & {$[0.023-0.025]$} & 358567 & 365410 & 364370 & 361066 \\
\hline Configural invariance & 6727.988* & 3924 & 0.962 & 0.945 & 0.027 & {$[0.026-0.028]$} & 357830 & 371516 & 369436 & 362828 \\
\hline Weak invariance & $7526.937^{*}$ & 4703 & 0.962 & 0.954 & 0.025 & {$[0.024-0.026]$} & 357485 & 366045 & 364744 & 360611 \\
\hline Strong invariance & $8003.023 *$ & 4766 & 0.957 & 0.948 & 0.026 & {$[0.025-0.027]$} & 357900 & 366046 & 364808 & 360875 \\
\hline Strict invariance & $8645.824 * *$ & 4842 & 0.949 & 0.940 & 0.028 & {$[0.027-0.029]$} & 358165 & 365810 & 364648 & 360956 \\
\hline Cor. uniqu. Invariance & $8178.084^{*}$ & 4872 & 0.956 & 0.948 & 0.026 & {$[0.025-0.027]$} & 358041 & 365489 & 364357 & 360761 \\
\hline Var-covar. Invariance & $8355.463 *$ & 4951 & 0.954 & 0.948 & 0.027 & {$[0.026-0.027]$} & 358131 & 365059 & 364006 & 360661 \\
\hline Latent means invariance & $8774.575^{*}$ & 4964 & 0.949 & 0.941 & 0.028 & {$[0.027-0.029]$} & 358567 & 365410 & 364370 & 361066 \\
\hline
\end{tabular}

Note. ICM= Independent cluster model; $\mathrm{CFA}=$ Confirmatory factor analysis; $\mathrm{H}=$ Hierarchical model; $\mathrm{B}=\mathrm{Bifactor}$ model; ESEM = Exploratory structural equation modeling; $\mathrm{df}=$ Degrees of freedom; $\mathrm{CFI}=$ comparative fit index; TLI = Tucker-Lewis index; RMSEA = root mean square error of approximation; $\mathrm{CI}=$ confidence interval; $\mathrm{AIC}=$ Akaike information criterion; $\mathrm{CAIC}=$ Constant $\mathrm{AIC} ; \mathrm{BIC}=$ Bayesian information criterion; $\mathrm{ABIC}=\mathrm{Sample}$ size adjusted $\mathrm{BIC}$; ESEM were estimated with target oblique rotation; bifactor-ESEM were estimated with bifactor orthogonal target rotation; $* p<.01$. 
Table 2. Standardized Factor Correlations for the CFA (above the diagonal) and ESEM (below the diagonal) solutions for the SDQ-I

\begin{tabular}{|c|c|c|c|c|c|c|c|c|c|c|c|}
\hline & $\begin{array}{l}\text { Global Self- } \\
\text { Esteem }\end{array}$ & Appearance & $\begin{array}{l}\text { Physical } \\
\text { Ability }\end{array}$ & Peer & Parent & $\begin{array}{l}\text { Academic } \\
\text { Competence }\end{array}$ & $\begin{array}{l}\text { Academic } \\
\text { Affect }\end{array}$ & $\begin{array}{l}\text { German } \\
\text { Competence }\end{array}$ & $\begin{array}{l}\text { German } \\
\text { Affect }\end{array}$ & $\begin{array}{l}\text { Math } \\
\text { Competence }\end{array}$ & $\begin{array}{l}\text { Math } \\
\text { Affect }\end{array}$ \\
\hline Global Self-Esteem & & $0.724 * *$ & $0.395 * *$ & $0.727 * *$ & $0.549 * *$ & $0.552 * *$ & $0.467 * *$ & $0.423 * *$ & $0.346 * *$ & $0.354 * *$ & $0.270 * *$ \\
\hline Appearance & $0.619 * *$ & & $0.341 * *$ & $0.619 * *$ & $0.355 * *$ & $0.348 * *$ & $0.309 * *$ & $0.278 * *$ & $0.238 * *$ & $0.200 * *$ & $0.170 * *$ \\
\hline Physical Ability & $0.329 * *$ & $0.300 * *$ & & $0.408 * *$ & $0.208 * *$ & $0.234 * *$ & $0.249 * *$ & $0.131 * *$ & $0.139 * *$ & $0.208 * *$ & $0.214 * *$ \\
\hline Peer & $0.565 * *$ & $0.497 * *$ & $0.370 * *$ & & $0.355 * *$ & $0.336 * *$ & $0.261 * *$ & $0.298 * *$ & $0.208 * *$ & $0.206^{* *}$ & $0.118 * *$ \\
\hline Parent & $0.506 * *$ & $0.324 * *$ & $0.218 * *$ & $0.311 * *$ & & $0.349 * *$ & $0.373 * *$ & $0.250 * *$ & $0.300 * *$ & $0.263 * *$ & $0.260 * *$ \\
\hline Academic Competence & $0.254 * *$ & $0.118 * *$ & $0.100 * *$ & $0.204 * *$ & 0.006 & & $0.733 * *$ & $0.722 * *$ & $0.508 * *$ & $0.636^{* *}$ & $0.450 * *$ \\
\hline Academic Affect & $0.215 * *$ & $0.133 * *$ & $0.147 * *$ & $0.063 * *$ & $0.311 * *$ & $0.110 * *$ & & $0.506 * *$ & $0.696 * *$ & $0.487 * *$ & $0.610 * *$ \\
\hline German Competence & $0.267 * *$ & $0.143 * *$ & $0.043 * *$ & $0.146 * *$ & $0.228 * *$ & $0.199 * *$ & $0.406 * *$ & & $0.781 * *$ & $0.251 * *$ & $0.106 * *$ \\
\hline German Affect & $0.219 * *$ & $0.122 * *$ & $0.109 * *$ & $0.142 * *$ & $0.180 * *$ & $0.270 * *$ & $0.343 * *$ & $0.393 * *$ & & $0.150 * *$ & $0.212 * *$ \\
\hline Math Competence & $0.260 * *$ & $0.111 * *$ & $0.169 * *$ & $0.100 * *$ & $0.254 * *$ & $0.235 * *$ & $0.360 * *$ & $0.282 * *$ & -0.016 & & $0.815 * *$ \\
\hline Math Affect & $0.216 * *$ & $0.145 * *$ & $0.208 * *$ & $0.089 * *$ & $0.225 * *$ & $0.198 * *$ & $0.352 * *$ & $-0.060 *$ & $0.172 * *$ & $0.648 * *$ & \\
\hline
\end{tabular}

Note. $* p<.05 . * * p<.01$ 
Table 3. Standardized Factor Loadings for Bifactor-ESEM Solution of the SDQ-I

\begin{tabular}{|c|c|c|c|c|c|c|c|c|c|c|c|c|c|}
\hline Items & $\begin{array}{l}\text { Global Self } \\
\text { Esteem }\end{array}$ & Appearance & $\begin{array}{l}\text { Physical } \\
\text { Ability }\end{array}$ & Peer & Parent & $\begin{array}{l}\text { Academic } \\
\text { Competence }\end{array}$ & $\begin{array}{l}\text { Academic } \\
\text { Affect }\end{array}$ & $\begin{array}{l}\text { German } \\
\text { Competence }\end{array}$ & $\begin{array}{l}\text { German } \\
\text { Affect }\end{array}$ & $\begin{array}{l}\text { Math } \\
\text { Competence }\end{array}$ & Math Affect & G-Factor & Uniquenesses \\
\hline 29 & 0.101 & $0.052 *$ & $0.053^{*}$ & $0.062 *$ & 0.000 & -0.027 & 0.023 & $-0.067^{*}$ & $0.099 * *$ & $-0.090 * *$ & -0.014 & $0.451 * *$ & $0.753 * *$ \\
\hline 37 & $0.277 * *$ & $0.216^{* *}$ & -0.013 & 0.119 ** & $0.160^{* *}$ & -0.033 & 0.042 & 0.026 & $-0.104 * *$ & 0.030 & -0.040 & 0.320 ** & $0.637 * *$ \\
\hline 45 & $0.381 * *$ & $0.294 * *$ & $0.088 * *$ & 0.031 & $0.202 * *$ & $-0.141 * *$ & 0.083 & -0.047 & -0.018 & 0.022 & 0.012 & $0.389 * *$ & $0.538 * *$ \\
\hline 61 & $0.273 * *$ & $0.053 *$ & -0.038 & $0.056^{*}$ & $0.198 * *$ & 0.008 & -0.010 & $0.128^{* *}$ & $-0.125^{* *}$ & 0.022 & -0.077 & $0.433 * *$ & $0.519 * *$ \\
\hline 67 & $0.306 * *$ & 0.025 & 0.027 & $0.110 * *$ & 0.012 & 0.070 & $-0.118 * *$ & $-0.056^{*}$ & $-0.069 * *$ & 0.013 & $-0.065 * *$ & $0.610 * *$ & $0.489 * *$ \\
\hline 70 & $0.280 * *$ & $0.100 * *$ & -0.023 & $0.254 * *$ & 0.034 & 0.056 & -0.061 & $-0.077^{*}$ & -0.025 & $-0.146^{* *}$ & -0.022 & $0.469 * *$ & $0.590 * *$ \\
\hline 72 & $0.411 * *$ & $0.281^{* *}$ & 0.036 & $0.098 * *$ & -0.003 & -0.017 & $-0.083 * *$ & $-0.089 * *$ & $-0.076^{* *}$ & $-0.121 * *$ & $-0.045^{*}$ & $0.557 * *$ & $0.393 * *$ \\
\hline 76 & $0.368^{* *}$ & $0.048^{*}$ & 0.025 & $0.051 *$ & 0.028 & -0.024 & $-0.064 *$ & $-0.077 * *$ & $-0.047 *$ & -0.025 & $-0.087 * *$ & $0.594 * *$ & $0.484 * *$ \\
\hline 1 & $0.072 * *$ & $0.629 * *$ & $0.056 * *$ & $0.094 * *$ & -0.004 & -0.015 & -0.007 & -0.017 & $-0.060 * *$ & $-0.045^{*}$ & -0.013 & $0.379 * *$ & $0.438 * *$ \\
\hline 8 & $0.175 * *$ & $0.589 * *$ & $0.102 * *$ & $0.083 * *$ & $0.074 * *$ & -0.086 & 0.060 & -0.010 & -0.026 & 0.005 & 0.029 & $0.392 * *$ & $0.433 * *$ \\
\hline 15 & 0.021 & $0.690 * *$ & 0.001 & $0.092 * *$ & 0.003 & -0.005 & -0.005 & -0.004 & 0.004 & $-0.058^{*}$ & $-0.064 * *$ & $0.449 * *$ & $0.305 * *$ \\
\hline 22 & 0.030 & $0.724 * *$ & $-0.020 * *$ & $0.077 * *$ & 0.011 & 0.026 & -0.015 & $-0.046^{*}$ & -0.021 & $-0.056^{* *}$ & $-0.069 * *$ & $0.478 * *$ & $0.229 * *$ \\
\hline 30 & 0.240 ** & $0.528 * *$ & 0.030 & $0.054 *$ & $0.128 * *$ & -0.080 & 0.023 & 0.002 & -0.038 & 0.035 & 0.010 & $0.376 * *$ & $0.452 * *$ \\
\hline 38 & -0.010 & $0.378 * *$ & $0.050^{*}$ & $0.281 * *$ & $-0.058 *$ & $0.116^{*}$ & $-0.117 * *$ & $-0.086^{*}$ & -0.038 & $-0.126^{* *}$ & $-0.062 *$ & $0.428 * *$ & $0.533 * *$ \\
\hline 46 & $0.097 *$ & $0.307 * *$ & $0.219^{* *}$ & 0.050 & 0.017 & $-0.100 *$ & -0.016 & -0.010 & $-0.066^{*}$ & $-0.054 *$ & $-0.055^{*}$ & $0.484 * *$ & $0.590 * *$ \\
\hline 54 & 0.009 & $0.365^{* *}$ & $0.054 *$ & $0.099 * *$ & $-0.099 * *$ & 0.086 & $-0.219 * *$ & $-0.169 * *$ & $-0.099 * *$ & $-0.069 * *$ & $-0.134 * *$ & $0.400 * *$ & $0.567 * *$ \\
\hline 62 & $0.216^{* *}$ & $0.350 * *$ & -0.011 & $0.074 * *$ & 0.029 & 0.038 & $-0.111 * *$ & -0.038 & -0.013 & $-0.119 * *$ & -0.032 & $0.441 * *$ & $0.599 * *$ \\
\hline 10 & 0.025 & 0.010 & $0.512 * *$ & 0.030 & $0.067 * *$ & $-0.138 * *$ & $0.098 *$ & -0.013 & 0.030 & -0.002 & 0.019 & $0.221 * *$ & $0.653 * *$ \\
\hline 17 & $0.119 * *$ & 0.024 & $0.744 * *$ & $0.095 * *$ & $0.068 * *$ & $0.342 * *$ & 0.127 & 0.009 & 0.011 & 0.044 & 0.052 & $0.118 * *$ & $0.267 * *$ \\
\hline 24 & $0.090 *$ & 0.024 & $0.783 * *$ & $0.095 * *$ & $0.047 *$ & $0.264 * *$ & 0.097 & -0.023 & 0.055 & 0.016 & 0.047 & $0.180 * *$ & $0.249 * *$ \\
\hline 32 & -0.005 & $0.073 * *$ & $0.351 * *$ & 0.055 & $-0.068 * *$ & -0.062 & $-0.164 * *$ & $-0.184 * *$ & -0.014 & -0.025 & -0.002 & $0.300 * *$ & $0.708^{* *}$ \\
\hline 40 & 0.010 & $0.045^{* *}$ & $0.809 * *$ & $0.059 * *$ & -0.008 & -0.058 & -0.030 & -0.020 & $-0.057 * *$ & -0.012 & 0.003 & 0.320 ** & $0.229 * *$ \\
\hline 48 & -0.049 & -0.026 & $0.575 * *$ & 0.025 & -0.021 & $-0.123 * *$ & $-0.106^{* *}$ & -0.046 & -0.050 & -0.004 & 0.002 & $0.403 * *$ & $0.472 * *$ \\
\hline 56 & -0.020 & $0.050^{* *}$ & $0.786 * *$ & 0.037 & $-0.043 * *$ & $-0.117 * *$ & -0.064 & -0.037 & $-0.063^{*}$ & $-0.062 * *$ & -0.029 & 0.399** & $0.189 * *$ \\
\hline 64 & -0.039 & 0.041 & $0.487 * *$ & 0.048 & -0.012 & $-0.100 *$ & -0.067 & $-0.150 * *$ & $-0.059 *$ & 0.037 & 0.038 & $0.320 * *$ & $0.612 * *$ \\
\hline 7 & $0.085^{*}$ & 0.070 ** & $0.127 * *$ & $\mathbf{0 . 5 8 9} * *$ & $0.116^{* *}$ & -0.117 & $0.120 * *$ & $0.056^{*}$ & -0.011 & 0.032 & $-0.051^{*}$ & $0.242 * *$ & $0.518^{* *}$ \\
\hline 14 & 0.009 & $0.051 *$ & $0.056^{* *}$ & $0.518^{* *}$ & -0.031 & -0.045 & 0.008 & $0.047 *$ & -0.025 & -0.011 & $-0.089 * *$ & $0.317 * *$ & $0.611^{* *}$ \\
\hline 21 & 0.028 & $0.055^{* *}$ & $0.101 * *$ & $0.575 * *$ & 0.042 & 0.010 & 0.010 & $0.086^{* *}$ & $-0.073 * *$ & 0.023 & $-0.108 * *$ & $0.271 * *$ & $0.523 * *$ \\
\hline 28 & 0.071 & 0.043 & $0.117 * *$ & $0.504 * *$ & $0.113 * *$ & $-0.112 *$ & $0.082 *$ & 0.019 & -0.016 & -0.017 & -0.012 & $0.364 * *$ & $0.560 * *$ \\
\hline 36 & $0.128 * *$ & $0.232 * *$ & 0.019 & $0.333 * *$ & -0.004 & -0.012 & -0.027 & -0.034 & $-0.075^{* *}$ & $-0.059 *$ & $-0.048^{*}$ & $0.398 * *$ & $0.647 * *$ \\
\hline 44 & 0.074 & $0.147 * *$ & 0.025 & $0.499 * *$ & 0.004 & 0.054 & $-0.107 * *$ & $-0.083 * *$ & -0.021 & $-0.097 * *$ & -0.042 & $0.448 * *$ & $0.490 * *$ \\
\hline 52 & 0.016 & $0.085^{* *}$ & $0.055^{* *}$ & $0.458 * *$ & $-0.044 *$ & 0.041 & $-0.135 * *$ & $-0.133 * *$ & -0.018 & -0.027 & $-0.058^{*}$ & 0.370 ** & $0.599 * *$ \\
\hline 60 & 0.073 & $0.112 * *$ & $0.060 * *$ & $0.454 * *$ & $-0.048^{*}$ & 0.088 & $-0.236^{* *}$ & $-0.098 * *$ & $-0.093 * *$ & $-0.060 *$ & $-0.090 * *$ & $0.486 * *$ & $0.441 * *$ \\
\hline 69 & $0.156^{* *}$ & $0.143 * *$ & $0.076^{* *}$ & $0.546 * *$ & -0.007 & 0.053 & $-0.123 * *$ & $-0.095 * *$ & $-0.051 *$ & $-0.118 * *$ & $-0.060 * *$ & $0.497 * *$ & $0.357 * *$ \\
\hline 5 & $0.086^{* *}$ & 0.027 & 0.024 & 0.022 & $0.542 * *$ & -0.028 & 0.038 & 0.002 & 0.038 & -0.005 & 0.012 & $0.387 * *$ & $0.543 * *$ \\
\hline 12 & $0.103 * *$ & -0.004 & $-0.058^{*}$ & 0.022 & $0.313 * *$ & 0.068 & 0.017 & $0.107 * *$ & -0.053 & $0.095 * *$ & -0.032 & $0.311 * *$ & $0.666^{* *}$ \\
\hline
\end{tabular}




\begin{tabular}{|c|c|c|c|c|c|c|c|c|c|c|c|c|c|}
\hline Items & $\begin{array}{l}\text { Global Self } \\
\text { Esteem }\end{array}$ & Appearance & $\begin{array}{l}\text { Physical } \\
\text { Ability }\end{array}$ & Peer & Parent & $\begin{array}{l}\text { Academic } \\
\text { Competence }\end{array}$ & $\begin{array}{l}\text { Academic } \\
\text { Affect }\end{array}$ & $\begin{array}{l}\text { German } \\
\text { Competence }\end{array}$ & $\begin{array}{l}\text { German } \\
\text { Affect }\end{array}$ & $\begin{array}{l}\text { Math } \\
\text { Competence }\end{array}$ & Math Affect & G-Factor & Uniquenesses \\
\hline 19 & 0.086 & $0.047 * *$ & 0.028 & -0.003 & $0.558 * *$ & -0.073 & 0.032 & -0.014 & $0.053 *$ & -0.005 & 0.016 & $0.281 * *$ & $0.589 * *$ \\
\hline 26 & $0.096^{*}$ & $0.088^{* *}$ & 0.013 & 0.042 & $0.535 * *$ & -0.011 & -0.018 & 0.009 & 0.032 & 0.017 & -0.012 & $0.302 * *$ & $0.601 * *$ \\
\hline 34 & 0.006 & -0.002 & -0.004 & $0.052 *$ & $0.413 * *$ & 0.046 & 0.019 & -0.017 & 0.001 & -0.040 & -0.019 & $0.347 * *$ & $0.702 * *$ \\
\hline 50 & 0.021 & -0.002 & -0.018 & -0.017 & $0.699 * *$ & -0.042 & -0.008 & $-0.050^{*}$ & 0.029 & 0.016 & 0.028 & $0.414 * *$ & $0.332 * *$ \\
\hline 58 & $0.059 *$ & 0.020 & -0.028 & -0.012 & $0.758 * *$ & $-0.060 * *$ & 0.017 & $-0.050 *$ & -0.014 & -0.021 & 0.004 & $0.423 * *$ & $0.235^{* *}$ \\
\hline 66 & 0.024 & -0.024 & 0.029 & 0.032 & $0.650 * *$ & 0.040 & $-0.079 *$ & $-0.078 * *$ & $-0.053 * *$ & $-0.090 * *$ & 0.019 & $0.432 * *$ & $0.363 * *$ \\
\hline 2 & $-0.102 * *$ & $-0.044 * *$ & $-0.074 * *$ & $-0.100 * *$ & $-0.068^{* *}$ & 0.099 & $0.199 * *$ & $0.267 * *$ & $-0.152 * *$ & $0.155^{* *}$ & $-0.074 * *$ & $0.604 * *$ & $0.429 * *$ \\
\hline 31 & $-0.067 *$ & $-0.131 * *$ & $-0.046^{*}$ & $-0.091 *$ & $-0.060 * *$ & 0.083 & $0.095 * *$ & $0.170 * *$ & $-0.053^{*}$ & $0.168 * *$ & $-0.055^{*} *$ & $0.628 * *$ & $0.491 * *$ \\
\hline 47 & $0.118^{* *}$ & -0.017 & -0.014 & -0.025 & $0.061^{*}$ & -0.011 & -0.001 & $0.220^{* *}$ & 0.013 & $0.131 * *$ & -0.025 & $0.446 * *$ & $0.550 * *$ \\
\hline 63 & $-0.070^{*}$ & $-0.122 * *$ & $-0.076^{* *}$ & $-0.112 * *$ & $-0.101 * *$ & 0.070 & $0.208^{* *}$ & $0.099 * *$ & $-0.075^{* *}$ & $0.154 * *$ & -0.042 & $0.691 * *$ & $0.386^{* *}$ \\
\hline 9 & $-0.071 * *$ & -0.011 & $-0.038^{*}$ & $-0.094 * *$ & 0.035 & -0.024 & $0.367 * *$ & -0.021 & $0.126^{* *}$ & $-0.065 * *$ & $0.134 * *$ & $0.585 \% *$ & $0.467 * *$ \\
\hline 23 & 0.062 & -0.011 & -0.008 & -0.020 & $0.092 * *$ & 0.061 & $0.174 * *$ & $0.085^{* *}$ & $0.188 * *$ & 0.007 & $0.180^{* *}$ & $0.372 * *$ & $0.624 * *$ \\
\hline 39 & $-0.087 * *$ & $-0.097 * *$ & -0.014 & $-0.128 * *$ & -0.058 & 0.067 & $0.406 * *$ & $-0.087 * *$ & $0.179 * *$ & -0.049 & $0.147 * *$ & $0.632 * *$ & $0.331 * *$ \\
\hline 55 & $-0.075^{* *}$ & $-0.097 * *$ & -0.027 & $-0.096^{* *}$ & $-0.037^{*}$ & 0.021 & $0.404 * *$ & $-0.102 * *$ & $0.232 * *$ & $-0.065^{* *}$ & $0.195^{* *}$ & $0.644 * *$ & $0.289 * *$ \\
\hline 71 & $-0.083 * *$ & $-0.131 * *$ & -0.021 & $-0.111 * *$ & $-0.050 * *$ & 0.051 & $0.418^{* *}$ & $-0.097 * *$ & $0.196^{* *}$ & 0.022 & $0.163^{* *}$ & $0.658 * *$ & $0.276^{* *}$ \\
\hline 4 & $-0.060 * *$ & $-0.041 *$ & $-0.117 * *$ & $-0.052^{* *}$ & $-0.054 * *$ & 0.050 & -0.029 & $0.596 * *$ & $0.106^{* *}$ & $-0.054^{*}$ & $-0.132 * *$ & $0.530 * *$ & $0.303 * *$ \\
\hline 18 & -0.081 & $-0.061 * *$ & $-0.086^{* *}$ & -0.021 & $-0.055^{* *}$ & 0.014 & -0.028 & $0.595 * *$ & $0.260 * *$ & -0.023 & $-0.151 * *$ & $0.548 * *$ & $0.233 * *$ \\
\hline 49 & -0.036 & $-0.091 * *$ & $-0.087 * *$ & -0.021 & $-0.064 * *$ & 0.005 & $-0.069 * *$ & $0.490 * *$ & $0.336^{* *}$ & -0.035 & $-0.145^{* *}$ & $0.530 * *$ & $0.317^{* *}$ \\
\hline 73 & -0.007 & $-0.113 * *$ & $-0.043^{*}$ & -0.036 & $-0.102 * *$ & 0.014 & -0.049 & $0.388 * *$ & $0.291 * *$ & -0.024 & $-0.187 * *$ & $0.597 * *$ & $0.343 * *$ \\
\hline 11 & $-0.063 * *$ & -0.004 & $-0.068 * *$ & $-0.054 * *$ & 0.001 & -0.032 & $0.122 * *$ & $0.284 * *$ & $0.595 * *$ & $-0.122 * *$ & $-0.047 * *$ & $0.461 * *$ & $0.309 * *$ \\
\hline 25 & $-0.070 * *$ & -0.026 & $-0.043 *$ & $-0.055^{* *}$ & 0.017 & -0.018 & $0.120^{* *}$ & $0.159 * *$ & $0.618^{* * *}$ & $-0.090 * *$ & -0.008 & $0.486 * *$ & $0.322 * *$ \\
\hline 41 & $-0.071 * *$ & $-0.069 * *$ & -0.008 & $-0.045^{* *}$ & 0.004 & -0.009 & $0.103 * *$ & $0.160^{* *}$ & $0.635^{* *}$ & $-0.124 * *$ & -0.030 & $0.515^{* *}$ & $0.268^{* *}$ \\
\hline 57 & -0.066 & $-0.073 * *$ & $-0.049 * *$ & $-0.076^{* *}$ & 0.003 & -0.021 & $0.118^{* *}$ & $0.175^{* *}$ & $0.651 * *$ & $-0.122 * *$ & -0.014 & $0.541 * *$ & $0.206^{* *}$ \\
\hline 65 & -0.003 & -0.087 & $-0.051 * *$ & $-0.065^{* *}$ & 0.009 & 0.016 & $0.071 * *$ & $0.233^{* *}$ & $0.566 * *$ & $-0.083 * *$ & $-0.040^{*}$ & $0.454 * *$ & $0.333 * *$ \\
\hline 13 & $-0.051 *$ & $-0.069 * *$ & -0.018 & $-0.034 *$ & $-0.041^{*}$ & 0.000 & -0.016 & $-0.071 * *$ & $-0.115^{* *}$ & $0.616 * *$ & $0.336^{* *}$ & $0.423 * *$ & $0.300 * *$ \\
\hline 27 & $-0.058 * *$ & $-0.054 * *$ & $-0.045^{* *}$ & $-0.055^{* *}$ & -0.028 & 0.029 & 0.004 & 0.036 & $-0.134 * *$ & $0.622 * *$ & $0.230^{* *}$ & $0.491 * *$ & $0.287 * *$ \\
\hline 43 & -0.028 & $-0.096 * *$ & 0.015 & -0.027 & -0.019 & -0.010 & -0.034 & $-0.048^{*}$ & $-0.102 * *$ & $0.557 * *$ & $0.344 * *$ & $0.543 * *$ & $0.251 * *$ \\
\hline 59 & $-0.036^{*}$ & $-0.072 * *$ & -0.017 & $-0.070 * *$ & $-0.036^{*}$ & 0.016 & -0.025 & -0.027 & $-0.098 * *$ & $0.630 * *$ & $0.347 * *$ & $0.542 * *$ & $0.164 * *$ \\
\hline 75 & 0.018 & $-0.073 * *$ & 0.009 & $-0.067 * *$ & 0.015 & 0.049 & -0.030 & -0.016 & $-0.108 * *$ & $0.572 * *$ & $0.315^{* *}$ & $0.457 * *$ & $0.309 * *$ \\
\hline 6 & -0.036 & $-0.043 * *$ & 0.016 & $-0.084 * *$ & $0.041^{*}$ & -0.013 & $0.048 * *$ & $-0.079 * *$ & $-0.078 * *$ & $0.298^{*}$ & $0.644 * *$ & $0.333 * *$ & $0.347 * *$ \\
\hline 20 & $-0.047 * *$ & $-0.046 * *$ & -0.005 & -0.094 & 0.011 & 0.005 & $0.113 * *$ & $-0.113 * *$ & -0.009 & $0.253 * *$ & $0.690 * *$ & $0.454 * *$ & $0.215^{* *}$ \\
\hline 35 & $-0.071^{* *}$ & $-0.045^{* *}$ & 0.026 & $-0.071 * *$ & -0.005 & 0.003 & $0.090 * *$ & $-0.118 * *$ & 0.001 & $0.265^{* *}$ & 0.690 ** & $0.464 * *$ & $0.204 * *$ \\
\hline 51 & -0.026 & $-0.043 * *$ & 0.025 & $-0.098 * *$ & $-0.016^{* *}$ & 0.011 & $0.076^{* *}$ & $-0.099 * *$ & $-0.037 * *$ & $0.275^{* *}$ & $0.763 * *$ & $0.460 * *$ & $0.100 * *$ \\
\hline 68 & $-0.060 * *$ & $-0.086^{* *}$ & $0.039 * *$ & $-0.062 * *$ & -0.013 & $-0.040^{*}$ & $0.095 * *$ & $-0.109 * *$ & 0.000 & $0.282 * *$ & $0.657 * *$ & $0.478 * *$ & $0.221 * *$ \\
\hline
\end{tabular}

Note. Negatively worded items are italicized; $* p<.05 . * * p<.01$ 
Table 4. Goodness of Fit Statistics and Information Criteria for the Models Estimated on the Simulated Data Set

\begin{tabular}{|c|c|c|c|c|c|c|c|c|c|c|}
\hline Model & $\chi^{2}$ & $\mathrm{df}$ & CFI & TLI & RMSEA & RMSEA 90\% CI & AIC & CAIC & $\mathrm{BIC}$ & $\mathrm{ABIC}$ \\
\hline ICM-CFA & $1020.469^{*}$ & 51 & 0.937 & 0.919 & 0.109 & {$[0.103-0.115]$} & 40905 & 41154 & 41115 & 40991 \\
\hline B-CFA & $661.772 *$ & 42 & 0.960 & 0.937 & 0.096 & {$[0.090-0.103]$} & 40564 & 40870 & 40822 & 40670 \\
\hline ESEM & $100.432 *$ & 33 & 0.996 & 0.991 & 0.036 & {$[0.028-0.044]$} & 40021 & 40385 & 40328 & 40146 \\
\hline B-ESEM & 30.139 & 24 & 1.000 & 0.999 & 0.013 & {$[0.000-0.025]$} & 39969 & 40390 & 40324 & 40114 \\
\hline Configural invariance & 65.071 & 48 & 0.999 & 0.997 & 0.021 & {$[0.000-0.033]$} & 39843 & 40685 & 40553 & 40134 \\
\hline Weak invariance & 98.538 & 80 & 0.999 & 0.998 & 0.017 & {$[0.000-0.026]$} & 39813 & 40451 & 40351 & 40033 \\
\hline Strong invariance & $173.907 *$ & 88 & 0.994 & 0.992 & 0.035 & {$[0.027-0.043]$} & 39872 & 40459 & 40367 & 40075 \\
\hline Partial Strong Invariance & 103.659 & 87 & 0.999 & 0.998 & 0.015 & {$[0.000-0.026]$} & 39804 & 40397 & 40304 & 40006 \\
\hline Strict invariance & 109.056 & 99 & 0.999 & 0.999 & 0.011 & {$[0.000-0.022]$} & 39785 & 40302 & 40221 & 39964 \\
\hline Var-covar. Invariance & 122.821 & 109 & 0.999 & 0.999 & 0.013 & {$[0.000-0.023]$} & 39779 & 40232 & 40161 & 39935 \\
\hline Latent means invariance & $252.702 *$ & 113 & 0.991 & 0.989 & 0.039 & {$[0.033-0.046]$} & 39901 & 40328 & 40261 & 40048 \\
\hline MIMIC (Null) & $235.810^{*}$ & 36 & 0.987 & 0.972 & 0.059 & {$[0.052-0.066]$} & 39969 & 40390 & 40324 & 40114 \\
\hline MIMIC (Saturated) & 30.894 & 78 & 1.000 & 0.999 & 0.013 & {$[0.000-0.026]$} & 39788 & 40285 & 40207 & 39959 \\
\hline MIMIC (Invariant) & $105.473 *$ & 70 & 0.995 & 0.989 & 0.038 & {$[0.030-0.046]$} & 39846 & 40293 & 40223 & 40000 \\
\hline MIMIC (Partial Invariance) & 36.184 & 31 & 1.000 & 0.999 & 0.010 & {$[0.000-0.022]$} & 39779 & 40232 & 40161 & 39935 \\
\hline Predictive Model (full med.) & $125.341 *$ & 81 & 0.998 & 0.996 & 0.018 & {$[0.012-0.025]$} & 56674 & 57229 & 57142 & 56865 \\
\hline Predictive Model (part. med.) & 73.479 & 80 & 1.000 & 1.001 & 0.000 & {$[0.000-0.011]$} & 56624 & 57185 & 57097 & 56818 \\
\hline
\end{tabular}

Note. $\mathrm{ICM}=$ Independent cluster model; $\mathrm{CFA}=$ Confirmatory factor analysis; $\mathrm{B}=$ Bifactor model; $\mathrm{ESEM}=$ Exploratory structural equation modeling; $\mathrm{df}=$

Degrees of freedom; $\mathrm{CFI}=$ comparative fit index; TLI = Tucker-Lewis index; RMSEA = root mean square error of approximation; $\mathrm{CI}=$ confidence interval; $\mathrm{AIC}=$ Akaike information criterion CAIC $=$ Constant $\mathrm{AIC} ; \mathrm{BIC}=$ Bayesian information criterion; $\mathrm{ABIC}=$ Sample size adjusted BIC ESEM were estimated with target oblique rotation; bifactor-ESEM were estimated with bifactor orthogonal target rotation;. $*: p<.01$. 
Table 5. Standardized Factor Correlations for the CFA (above the diagonal) and ESEM (below the diagonal) solutions for the Simulated Data Set

\begin{tabular}{llll} 
& Factor 1 & Factor 2 & Factor 3 \\
\hline Factor 1 & & $0.516^{* *}$ & $0.613^{* *}$ \\
Factor 2 & $0.475^{* *}$ & & $0.731^{* *}$ \\
Factor 3 & $0.522^{* *}$ & $0.629^{* *}$ & \\
\hline
\end{tabular}

Note. ${ }^{*} p<.05 . * * p<.01$ 
Table 6. Standardized Factor Loadings for Bifactor-ESEM Solution for the Simulated Data

\begin{tabular}{|c|c|c|c|c|c|}
\hline Items & $\begin{array}{l}\text { G-Factor } \\
\text { Loadings }\end{array}$ & $\begin{array}{l}\text { S-Factor1 } \\
\text { Loadings }\end{array}$ & $\begin{array}{l}\text { S-Factor2 } \\
\text { Loadings }\end{array}$ & $\begin{array}{l}\text { S-Factor3 } \\
\text { Loadings }\end{array}$ & Uniquenesses \\
\hline $\mathrm{X} 1$ & $0.469 * *$ & $0.602 * *$ & $-0.063^{*}$ & $0.100 * *$ & $0.403 * *$ \\
\hline $\mathrm{X} 2$ & $0.466 * *$ & $0.691 * *$ & $0.176 * *$ & 0.008 & $0.273 * *$ \\
\hline X3 & $0.663 * *$ & $0.521 *$ & $-0.176^{* *}$ & -0.023 & $0.258 * *$ \\
\hline $\mathrm{X} 4$ & $0.639 * *$ & $0.556 * *$ & $-0.050^{*}$ & $-0.096^{* *}$ & $0.271 * *$ \\
\hline Y1 & $0.661 * *$ & -0.023 & $0.533 * *$ & $0.134 * *$ & $0.261 * *$ \\
\hline Y2 & $0.692 * *$ & $0.130 * *$ & $0.477 * *$ & -0.015 & $0.276 * *$ \\
\hline Y3 & $0.747 * *$ & $-0.119 * *$ & $0.546 * *$ & 0.005 & $0.130 * *$ \\
\hline Y4 & $0.745 * *$ & $-0.042 * *$ & $0.498 * *$ & $-0.075 * *$ & $0.190 * *$ \\
\hline $\mathrm{Z1}$ & $0.789 * *$ & $0.128 * *$ & -0.006 & $0.369 * *$ & $0.225 * *$ \\
\hline $\mathrm{Z2}$ & $0.791 * *$ & -0.011 & $0.174 * *$ & $0.353 * *$ & $0.219 * *$ \\
\hline $\mathrm{Z3}$ & $0.659 * *$ & 0.005 & $-0.087 * *$ & $0.552 * *$ & $0.253 * *$ \\
\hline Z4 & $0.644 * *$ & $-0.079 * *$ & $0.031 *$ & $0.583 * *$ & $0.238 * *$ \\
\hline
\end{tabular}

$* p<.05 . * * p<.01$ 
Online Supplements for:

A Bifactor Exploratory Structural Equation Modeling Framework for the Identification of Distinct Sources of Construct-Relevant Psychometric Multidimensionality

\section{Authors' note:}

These online supplements are to be posted on the journal website and hot-linked to the manuscript. If the journal does not offer this possibility, these materials can alternatively be posted on one of our personal websites (we will adjust the in-text reference upon acceptance).

Table S1.

Goodness of Fit Statistics and Information Criteria for the Models Estimated on the full SDQ-I and Excluding the Method Factor (Negative Items) and the Correlated Uniquenesses (Parallel Items).

\begin{tabular}{|c|c|c|c|c|c|c|c|c|c|c|}
\hline Model & $\chi^{2}$ & df & CFI & TLI & RMSEA & RMSEA 90\% CI & AIC & CAIC & $\mathrm{BIC}$ & SBIC \\
\hline ICM-CFA & 9711.995* & 2719 & 0.904 & 0.900 & 0.036 & $0.035-0.037$ & 364067 & 365929 & 365646 & 364747 \\
\hline $\mathrm{H}-\mathrm{CFA}$ & $14230.037 *$ & 2763 & 0.843 & 0.838 & 0.046 & $0.045-0.047$ & 369586 & 371158 & 370919 & 370160 \\
\hline B-CFA & $13480.732 *$ & 2698 & 0.852 & 0.844 & 0.045 & $0.044-0.046$ & 368771 & 370771 & 370467 & 369501 \\
\hline ESEM & $5152.860 *$ & 2069 & 0.958 & 0.942 & 0.028 & $0.027-0.029$ & 359601 & 365740 & 364807 & 361843 \\
\hline H-ESEM & $6087.575^{*}$ & 2113 & 0.946 & 0.927 & 0.031 & $0.030-0.032$ & 360678 & 366527 & 365638 & 362814 \\
\hline B-ESEM & $4894.806^{*}$ & 2004 & 0.960 & 0.944 & 0.027 & $0.026-0.028$ & 359229 & 365795 & 364797 & 361627 \\
\hline
\end{tabular}

Note $. \mathrm{df}=$ Degrees of freedom; CFI = comparative fit index; TLI = Tucker-Lewis index; RMSEA = root mean square error of approximation; CI = confidence interval; $\mathrm{AIC}=$ Akaike information criterion; $\mathrm{CAIC}=$ Constant $\mathrm{AIC}$; BIC $=$ Bayesian information criterion; $\mathrm{ABIC}=\mathrm{Sample}$ size adjusted $\mathrm{BIC}$. ESEM models were conducted with target oblique rotation. ${ }^{*}$ All $\chi^{2}$ values are significant $(p<.01)$. 
Table S2.

Standardized Factor Loadings for the First-Order CFA and ESEM Solutions for the SDQ-I

\begin{tabular}{|c|c|c|c|c|c|c|c|c|c|c|c|c|c|c|}
\hline \multirow[b]{2}{*}{ Items } & \multirow[b]{2}{*}{$\begin{array}{l}\text { Global Self } \\
\text { Esteem }\end{array}$} & \multirow[b]{2}{*}{ Appearance } & \multirow[b]{2}{*}{$\begin{array}{l}\text { Physical } \\
\text { Ability }\end{array}$} & \multirow[b]{2}{*}{ Peer } & \multirow[b]{2}{*}{ Parent } & \multicolumn{3}{|c|}{ First-Order ESEM Solution } & \multirow[b]{2}{*}{$\begin{array}{l}\text { German } \\
\text { Affect }\end{array}$} & \multirow[b]{2}{*}{$\begin{array}{l}\text { Math } \\
\text { Competence }\end{array}$} & \multirow[b]{2}{*}{$\begin{array}{l}\text { Math } \\
\text { Affect }\end{array}$} & \multirow[b]{2}{*}{ Uniquenesses } & \multicolumn{2}{|c|}{ First-Order CFA Solution } \\
\hline & & & & & & $\begin{array}{l}\text { Academic } \\
\text { Competence }\end{array}$ & $\begin{array}{l}\text { Academic } \\
\text { Affect }\end{array}$ & $\begin{array}{l}\text { German } \\
\text { Competence }\end{array}$ & & & & & $\begin{array}{l}\text { Factor } \\
\text { loadings }\end{array}$ & Uniquenesses \\
\hline 29 & $0.239 * *$ & 0.060 & $0.073 * *$ & $0.075^{*}$ & 0.024 & 0.070 & $0.106^{* *}$ & -0.041 & $0.158 * *$ & -0.037 & 0.034 & $0.755^{* *}$ & $0.457 * *$ & $0.791 * *$ \\
\hline 37 & $0.312 * *$ & $0.165^{* *}$ & -0.051 & 0.073 & $0.104 * *$ & $-0.115^{*}$ & 0.060 & 0.029 & $-0.118^{* *}$ & 0.051 & -0.046 & $0.642 * *$ & $0.457 * *$ & $0.680 * *$ \\
\hline 45 & $0.435 * *$ & $0.239 * *$ & $0.057 *$ & -0.079 & 0.114 & -0.224 & 0.093 & -0.074 & -0.006 & 0.066 & -0.036 & $0.558^{* *}$ & $0.570 * *$ & $0.675^{* *}$ \\
\hline 53 & $0.515 * *$ & -0.016 & $0.064 * *$ & $0.092 * *$ & $0.141 * *$ & -0.065 & 0.059 & -0.008 & -0.002 & 0.007 & -0.004 & $0.547 * *$ & $0.661 * *$ & $0.563 * *$ \\
\hline 61 & $0.407 * *$ & -0.011 & -0.033 & 0.028 & $0.191 * *$ & -0.004 & 0.022 & $0.188 * *$ & $-0.151 * *$ & 0.023 & -0.023 & $0.520 * *$ & $0.505^{* *}$ & $0.556^{* *}$ \\
\hline 67 & $\mathbf{0 . 5 8 0} * *$ & -0.041 & $0.045^{*}$ & $0.099 * *$ & 0.020 & $0.117 *$ & -0.021 & 0.006 & 0.014 & $0.091 *$ & -0.005 & $0.492 * *$ & $0.695 * *$ & $0.518^{* *}$ \\
\hline 70 & $0.464 * *$ & 0.000 & $-0.054 *$ & $0.268^{* *}$ & 0.005 & 0.006 & 0.037 & -0.003 & -0.004 & $-0.147 * *$ & $0.104 *$ & $0.591 * *$ & $0.605^{* *}$ & $0.634^{* *}$ \\
\hline 72 & $0.651 * *$ & $0.221^{* *}$ & 0.027 & 0.008 & $-0.058^{*}$ & -0.010 & 0.007 & -0.010 & -0.031 & $-0.098 * *$ & $0.064 *$ & $0.393^{* *}$ & $0.738 * *$ & $0.456^{* *}$ \\
\hline 74 & $0.642 * *$ & 0.002 & 0.018 & 0.049 & $-0.058 *$ & 0.083 & -0.042 & 0.047 & 0.030 & $0.081^{*}$ & -0.058 & $0.504 * *$ & $0.676^{* *}$ & $0.542 * *$ \\
\hline 76 & $0.668 * *$ & -0.022 & $0.047 *$ & 0.004 & 0.016 & 0.069 & 0.037 & -0.038 & 0.036 & 0.061 & -0.047 & $0.486^{* *}$ & $0.700 * *$ & $0.510^{* *}$ \\
\hline 1 & -0.033 & $0.762 * *$ & 0.024 & 0.016 & -0.029 & -0.038 & 0.014 & 0.026 & -0.039 & -0.015 & 0.031 & $0.438^{* *}$ & $0.742 * *$ & $0.450^{* *}$ \\
\hline 8 & 0.071 & $0.673 * *$ & $0.060 * *$ & -0.014 & 0.009 & $-0.165^{*}$ & 0.057 & -0.002 & -0.015 & 0.042 & 0.021 & $0.437 * *$ & $0.725^{* *}$ & $0.475 * *$ \\
\hline 15 & $-0.103 * *$ & $0.874 * *$ & -0.030 & 0.027 & 0.006 & 0.001 & 0.026 & 0.025 & $0.051 *$ & 0.019 & -0.032 & $0.305 * *$ & $0.813 * *$ & $0.339 * *$ \\
\hline 22 & -0.078 & $0.917 * *$ & $-0.053 * *$ & 0.006 & 0.023 & 0.036 & 0.030 & -0.019 & 0.037 & 0.029 & -0.040 & $0.234 * *$ & $0.855^{* *}$ & $0.269 * *$ \\
\hline 30 & $0.181 * *$ & $0.580 * *$ & -0.013 & -0.054 & 0.061 & $-0.176^{*}$ & 0.013 & 0.005 & -0.023 & $0.082 *$ & -0.009 & $0.458^{* *}$ & $0.662 * *$ & $0.490^{* *}$ \\
\hline 38 & -0.031 & $0.448^{* *}$ & 0.020 & $0.306^{* *}$ & -0.019 & 0.135 & -0.045 & -0.012 & 0.022 & -0.087 & 0.051 & $0.548 * *$ & $0.613 * *$ & $0.625^{* *}$ \\
\hline 46 & $0.137^{*}$ & 0.371 ** & $0.252 * *$ & -0.007 & 0.036 & 0.049 & 0.031 & 0.050 & -0.022 & -0.013 & -0.004 & $0.599 * *$ & $0.574 * *$ & $0.671^{* *}$ \\
\hline 54 & 0.070 & $0.482 * *$ & $0.063 * *$ & 0.059 & -0.015 & $0.269 * *$ & $-0.153 * *$ & $-0.114 * *$ & 0.036 & 0.032 & -0.062 & $0.562 * *$ & $0.572 * *$ & $0.673 * *$ \\
\hline 62 & $0.316^{* *}$ & $0.371 * *$ & -0.025 & 0.006 & 0.020 & 0.018 & -0.060 & 0.044 & 0.023 & $-0.105^{*}$ & $0.082 *$ & $0.601 * *$ & $0.593 * *$ & $0.648^{* *}$ \\
\hline 3 & -0.082 & $0.119 * *$ & $0.706 * *$ & 0.019 & -0.009 & 0.058 & -0.060 & $0.104 * *$ & -0.028 & -0.044 & 0.034 & $0.464^{* *}$ & $0.721^{* *}$ & $0.480^{* *}$ \\
\hline 10 & -0.014 & -0.018 & $0.559 * *$ & -0.011 & 0.052 & -0.095 & 0.094 & -0.019 & 0.031 & 0.007 & -0.029 & $0.667 * *$ & $0.554 * *$ & $0.693 * *$ \\
\hline 17 & 0.018 & $-0.104 * *$ & $0.726 * *$ & 0.040 & -0.033 & -0.154 & 0.109 & -0.029 & -0.069 & -0.009 & -0.032 & $0.470 * *$ & $0.681 * *$ & $0.531 * *$ \\
\hline 24 & 0.013 & $-0.088^{*}$ & $0.785 * *$ & 0.042 & -0.035 & -0.143 & 0.095 & -0.044 & 0.001 & -0.015 & -0.026 & $0.389 * *$ & $0.749 * *$ & $0.438^{* *}$ \\
\hline 32 & 0.067 & $0.098 * *$ & $0.399 * *$ & 0.014 & -0.008 & 0.133 & $-0.139 *$ & $-0.141 * *$ & $0.106^{* *}$ & 0.039 & 0.025 & $0.706^{* *}$ & $0.470 * *$ & $0.779 * *$ \\
\hline 40 & -0.002 & 0.004 & $0.902 * *$ & -0.010 & -0.018 & -0.040 & -0.018 & 0.043 & $-0.045^{*}$ & -0.011 & -0.004 & $0.215^{* *}$ & $0.883 * *$ & $0.221 * *$ \\
\hline 48 & 0.005 & -0.007 & $0.669 * *$ & -0.008 & 0.055 & 0.133 & -0.094 & 0.037 & 0.024 & 0.018 & 0.048 & $0.486^{* *}$ & $0.693 * *$ & $0.520 * *$ \\
\hline 56 & 0.013 & $0.046^{*}$ & $0.894 * *$ & -0.030 & -0.011 & 0.056 & -0.034 & $0.051 *$ & -0.022 & -0.053 & 0.007 & $0.196^{* *}$ & $0.895 * *$ & $0.198^{* *}$ \\
\hline 64 & -0.024 & $0.066^{*}$ & $0.546 * *$ & 0.015 & 0.044 & 0.094 & -0.053 & $-0.120 * *$ & 0.032 & 0.083 & 0.024 & $0.616^{* *}$ & $0.595^{* *}$ & $0.646^{* *}$ \\
\hline 7 & -0.077 & -0.050 & 0.039 & $0.703 * *$ & 0.044 & $-0.224^{*}$ & $0.114^{*}$ & 0.010 & -0.028 & $0.082 *$ & $-0.090^{*}$ & $0.536 * *$ & $0.571 * *$ & $0.673 * *$ \\
\hline 14 & -0.062 & -0.006 & 0.004 & $0.651 * *$ & -0.040 & -0.015 & 0.038 & 0.059 & -0.002 & 0.044 & -0.048 & $0.617 * *$ & $0.582 * *$ & $0.661 * *$ \\
\hline 21 & $-0.096^{*}$ & -0.036 & 0.031 & $0.710 * *$ & 0.015 & -0.070 & 0.023 & $0.089 * *$ & $-0.080^{* *}$ & 0.068 & $-0.091^{*}$ & $0.522 * *$ & $0.592 * *$ & $0.616^{* *}$ \\
\hline 28 & -0.009 & -0.049 & $0.058^{*}$ & $0.613^{* *}$ & 0.087 & -0.119 & $0.106^{*}$ & 0.023 & -0.018 & 0.008 & 0.012 & $0.576^{* *}$ & $0.596 * *$ & $0.644 * *$ \\
\hline 36 & $0.139 * *$ & $0.212 * *$ & -0.025 & $0.370 * *$ & -0.018 & 0.009 & 0.025 & 0.010 & -0.047 & -0.030 & 0.027 & $0.648^{* *}$ & $0.578 * *$ & $0.666^{* *}$ \\
\hline 44 & 0.082 & $0.093^{*}$ & -0.036 & $0.599 * *$ & 0.020 & 0.052 & -0.043 & -0.025 & 0.038 & -0.057 & 0.069 & $0.491^{* *}$ & $0.706^{* *}$ & $0.501^{* *}$ \\
\hline 52 & 0.018 & 0.050 & 0.008 & $0.560 * *$ & -0.009 & $0.098 *$ & $-0.084 *$ & $-0.111 * *$ & $0.084 *$ & 0.060 & -0.005 & $0.596^{* *}$ & $0.609 * *$ & $0.629 * *$ \\
\hline 60 & $0.147 *$ & 0.070 & 0.029 & $0.535^{* *}$ & 0.006 & $0.173 *$ & $-0.172 *$ & -0.011 & 0.009 & 0.005 & 0.037 & $0.442 * *$ & $0.723 * *$ & $0.477 * *$ \\
\hline 69 & $0.211^{* *}$ & 0.045 & 0.014 & $0.635^{* *}$ & -0.014 & 0.028 & -0.044 & -0.026 & 0.006 & $-0.080 *$ & 0.064 & $0.359^{* *}$ & $0.808 * *$ & $0.348^{* *}$ \\
\hline
\end{tabular}




\begin{tabular}{|c|c|c|c|c|c|c|c|c|c|c|c|c|c|c|}
\hline \multirow[b]{2}{*}{ Items } & \multirow[b]{2}{*}{$\begin{array}{l}\text { Global Self } \\
\text { Esteem }\end{array}$} & \multirow[b]{2}{*}{ Appearance } & \multirow[b]{2}{*}{$\begin{array}{l}\text { Physical } \\
\text { Ability }\end{array}$} & \multirow[b]{2}{*}{ Peer } & \multirow[b]{2}{*}{ Parent } & \multicolumn{3}{|c|}{ First-Order ESEM Solution } & \multirow[b]{2}{*}{$\begin{array}{l}\text { German } \\
\text { Affect }\end{array}$} & \multirow[b]{2}{*}{$\begin{array}{l}\text { Math } \\
\text { Competence }\end{array}$} & \multirow[b]{2}{*}{$\begin{array}{l}\text { Math } \\
\text { Affect }\end{array}$} & \multirow[b]{2}{*}{ Uniquenesses } & \multicolumn{2}{|c|}{ First-Order CFA Solution } \\
\hline & & & & & & $\begin{array}{l}\text { Academic } \\
\text { Competence }\end{array}$ & $\begin{array}{l}\text { Academic } \\
\text { Affect }\end{array}$ & $\begin{array}{l}\text { German } \\
\text { Competence }\end{array}$ & & & & & $\begin{array}{l}\text { Factor } \\
\text { loadings }\end{array}$ & Uniquenesses \\
\hline 5 & 0.042 & 0.004 & 0.021 & 0.006 & $0.627 * *$ & -0.048 & 0.029 & 0.003 & 0.033 & 0.005 & -0.001 & $0.542 * *$ & $0.675^{* *}$ & $0.544 * *$ \\
\hline 12 & 0.114 & -0.026 & $-0.060 *$ & 0.030 & $\mathbf{0 . 3 5 0}$ ** & -0.003 & 0.019 & $0.117 * *$ & $-0.072 *$ & $0.118^{*}$ & -0.052 & $0.668^{* *}$ & $0.388 * *$ & $0.722 * *$ \\
\hline 19 & 0.013 & 0.020 & 0.021 & -0.041 & $0.625 * *$ & -0.113 & 0.005 & -0.032 & 0.048 & 0.011 & -0.024 & $0.589 * *$ & $0.628 * *$ & $0.606^{* *}$ \\
\hline 26 & 0.019 & $0.058^{*}$ & -0.002 & 0.010 & $0.597 * *$ & -0.102 & -0.046 & 0.001 & 0.032 & 0.047 & -0.047 & $0.600 * *$ & $0.620 * *$ & $0.615^{* *}$ \\
\hline 34 & -0.016 & -0.004 & -0.001 & $0.073 *$ & $0.516 * *$ & 0.067 & 0.043 & -0.001 & 0.001 & -0.036 & 0.001 & $0.704 * *$ & $0.539 * *$ & $0.710 * *$ \\
\hline 42 & -0.005 & -0.011 & 0.023 & 0.073 & $0.649 * *$ & 0.133 & -0.019 & -0.065 & 0.033 & -0.020 & -0.008 & $0.549^{* *}$ & $0.657^{* *}$ & $0.568 * *$ \\
\hline 50 & -0.051 & 0.003 & -0.015 & -0.032 & $0.853 * *$ & 0.015 & -0.032 & -0.050 & $0.051^{*}$ & 0.036 & 0.006 & $0.330^{* *}$ & $0.812 * *$ & $0.341^{* *}$ \\
\hline 58 & -0.008 & 0.011 & $-0.028 *$ & -0.038 & $0.908 * *$ & 0.003 & 0.002 & $-0.047 *$ & -0.011 & -0.018 & -0.007 & $0.235^{* *}$ & $0.864 * *$ & $0.254^{* *}$ \\
\hline 66 & 0.011 & -0.047 & $0.041^{*}$ & 0.024 & $0.813 * *$ & 0.104 & -0.068 & -0.010 & -0.051 & $-0.131 * *$ & $0.096^{* *}$ & $0.370 * *$ & $0.779 * *$ & $0.393 * *$ \\
\hline 2 & 0.013 & $0.103^{* *}$ & 0.015 & 0.007 & 0.030 & $0.333 * *$ & $0.313^{* *}$ & $0.333^{* *}$ & $-0.179 * *$ & $0.185 * *$ & -0.048 & $0.430 * *$ & $0.738^{* *}$ & $0.456^{* *}$ \\
\hline 16 & -0.019 & $0.069 * *$ & 0.030 & 0.039 & $0.078 * *$ & $0.370 * *$ & $0.312 * *$ & $0.377 * *$ & $-0.205^{* *}$ & $0.151^{* *}$ & -0.020 & $0.370^{* *}$ & $0.765^{* *}$ & $0.414 * *$ \\
\hline 31 & $0.106^{* *}$ & -0.017 & $0.046^{*}$ & 0.008 & $0.051 *$ & $0.329 * *$ & $0.196^{* *}$ & $0.221 * *$ & -0.011 & $0.249 * *$ & -0.048 & $0.491^{* *}$ & $0.716^{* *}$ & $0.487^{* *}$ \\
\hline 47 & $0.221 * *$ & -0.005 & 0.027 & -0.010 & $0.071 *$ & 0.014 & 0.010 & $0.272 * *$ & 0.018 & $0.182 * *$ & -0.012 & $0.555^{* *}$ & $0.461 * *$ & $0.574 * *$ \\
\hline 63 & $0.135^{* *}$ & 0.017 & 0.015 & 0.004 & 0.013 & $0.382 * *$ & $0.359 * *$ & $0.124 * *$ & -0.029 & $0.242 * *$ & -0.054 & $0.384 * *$ & $0.791 * *$ & $0.375^{* *}$ \\
\hline 9 & 0.029 & $0.104 * *$ & 0.006 & 0.001 & $0.102 * *$ & $0.153^{*}$ & $0.514 * *$ & -0.013 & $0.112 * *$ & $-0.077^{*}$ & $0.161 * *$ & $0.472 * *$ & $0.717 * *$ & $0.486 * *$ \\
\hline 23 & 0.101 & -0.009 & -0.008 & 0.025 & 0.072 & -0.090 & $0.211 * *$ & $0.109 * *$ & $0.145^{* *}$ & -0.029 & $0.208^{* *}$ & $0.626^{* *}$ & $0.479 * *$ & $0.626^{* *}$ \\
\hline 39 & $0.083^{* *}$ & 0.019 & $0.044 * *$ & -0.007 & 0.011 & $0.220 * *$ & $0.601 * *$ & $-0.104 * *$ & $0.191^{* *}$ & -0.030 & $0.142 * *$ & $0.331 * *$ & $0.815^{* *}$ & $0.336^{* *}$ \\
\hline 55 & $0.080 *$ & 0.009 & 0.019 & 0.030 & 0.028 & $0.174 * *$ & $0.583 * *$ & $-0.118 * *$ & $0.250 * *$ & -0.055 & $0.205^{* *}$ & $0.289 * *$ & $0.844 * *$ & $0.288 * *$ \\
\hline 71 & $0.081^{* *}$ & -0.017 & $0.031 *$ & 0.025 & 0.017 & $0.203^{* *}$ & $0.605 * *$ & $-0.141 * *$ & $0.230 * *$ & $0.075^{*}$ & $0.115^{* *}$ & $0.276^{* *}$ & $0.849^{* *}$ & $0.279 * *$ \\
\hline 4 & 0.018 & $0.056^{*}$ & -0.019 & 0.022 & 0.025 & $0.176^{* *}$ & -0.026 & $0.790 * *$ & 0.015 & $-0.111 * *$ & $0.099 * *$ & $0.304 * *$ & $0.785^{* *}$ & $0.383^{* *}$ \\
\hline 18 & -0.033 & $0.040^{*}$ & 0.010 & $0.066 * *$ & 0.018 & $0.111 * *$ & $-0.044 *$ & $0.739 * *$ & $0.213 * *$ & -0.006 & 0.017 & $0.233 * *$ & $0.862 * *$ & $0.257 * *$ \\
\hline 33 & $0.075^{*}$ & 0.038 & 0.008 & 0.003 & 0.030 & 0.034 & -0.064 & $0.658^{* *}$ & $0.182 * *$ & -0.008 & $0.067 *$ & $0.365^{* *}$ & $0.761^{* *}$ & $0.368^{* *}$ \\
\hline 49 & 0.052 & -0.013 & 0.001 & $0.051 *$ & 0.004 & $0.096^{*}$ & $-0.082 * *$ & $0.601 * *$ & $0.337 * *$ & 0.021 & -0.005 & $0.317 * *$ & $0.841 * *$ & $0.293 * *$ \\
\hline 73 & $0.152 * *$ & -0.035 & $0.057 * *$ & 0.033 & -0.025 & $0.168 * *$ & -0.015 & $0.471 * *$ & $0.332 * *$ & $0.079 *$ & $-0.088^{*}$ & $0.342 * *$ & $0.813 * *$ & $0.340 * *$ \\
\hline 11 & -0.046 & $0.090^{* *}$ & -0.019 & 0.012 & 0.029 & $-0.076^{*}$ & $0.141^{* *}$ & $0.278^{* *}$ & 0.620 ** & -0.020 & -0.017 & $0.309^{* *}$ & $0.829 * *$ & $0.312^{* *}$ \\
\hline 25 & -0.033 & $0.066^{* *}$ & 0.001 & 0.016 & $0.054^{*}$ & $-0.066^{* *}$ & $0.160 * *$ & $0.120 * *$ & $0.687 * *$ & 0.053 & -0.028 & $0.322 * *$ & $0.820 * *$ & $0.327 * *$ \\
\hline 41 & -0.009 & 0.014 & $0.046 * *$ & 0.031 & $0.054 * *$ & -0.028 & $0.149 * *$ & $0.132 * *$ & $0.709 * *$ & 0.014 & -0.027 & $0.268 * *$ & $0.851 * *$ & $0.276^{* *}$ \\
\hline 57 & 0.012 & 0.019 & 0.009 & 0.002 & $0.055^{* *}$ & -0.028 & $0.167 * *$ & $0.152 * *$ & $0.723 * *$ & 0.014 & -0.005 & $0.205^{* *}$ & $0.892 * *$ & $0.204 * *$ \\
\hline 65 & $0.078^{*}$ & -0.037 & -0.001 & -0.007 & 0.031 & -0.065 & $0.097 * *$ & $0.221 * *$ & $0.610 * *$ & 0.033 & -0.029 & $0.333 * *$ & $0.777 * *$ & $0.339 * *$ \\
\hline 13 & -0.026 & 0.020 & -0.010 & $0.048^{*}$ & -0.013 & 0.045 & -0.041 & $-0.104 * *$ & 0.025 & $0.764 * *$ & $0.140 * *$ & $0.300 * *$ & $0.828^{* *}$ & $0.315 * *$ \\
\hline 27 & -0.025 & $0.058 * *$ & -0.019 & 0.034 & 0.011 & $0.087^{*}$ & -0.006 & 0.004 & -0.008 & $0.798 * *$ & 0.026 & $0.286^{* *}$ & $0.826^{* *}$ & $0.317^{* *}$ \\
\hline 43 & 0.041 & -0.017 & $0.040 * *$ & $0.057 * *$ & 0.029 & 0.080 & -0.048 & -0.037 & 0.031 & $0.676 * *$ & $0.210 * *$ & $0.251 * *$ & $0.867 * *$ & $0.249 * *$ \\
\hline 59 & 0.027 & 0.030 & 0.010 & 0.013 & 0.001 & $0.066^{* *}$ & $-0.040^{*}$ & -0.035 & $0.043 *$ & $0.782 * *$ & $0.168^{* *}$ & $0.164^{* *}$ & $0.917^{* *}$ & $0.159^{* *}$ \\
\hline 75 & 0.077 & -0.016 & 0.026 & -0.010 & 0.030 & 0.016 & -0.052 & -0.025 & 0.001 & $0.693 * *$ & $0.148^{* *}$ & $0.310^{* *}$ & $0.804 * *$ & $0.316^{* *}$ \\
\hline 6 & -0.031 & -0.005 & 0.008 & -0.031 & $0.047^{*}$ & $-0.062 * *$ & 0.010 & 0.045 & $-0.094 * *$ & $0.140^{* *}$ & $0.714 * *$ & $0.347^{* *}$ & $0.792 * *$ & $0.359^{* *}$ \\
\hline 20 & -0.004 & 0.012 & -0.008 & -0.017 & 0.025 & -0.027 & $0.114 * *$ & 0.015 & -0.021 & $0.090 * *$ & $0.781 * *$ & $0.215^{* *}$ & $0.886^{* *}$ & $0.216^{* *}$ \\
\hline 35 & -0.038 & 0.018 & 0.027 & 0.012 & 0.014 & -0.024 & $0.088^{* *}$ & 0.013 & 0.001 & $0.115^{* *}$ & $0.777 * *$ & $0.204 * *$ & $0.892 * *$ & $0.204 * *$ \\
\hline 51 & 0.026 & 0.003 & $0.022 *$ & -0.029 & -0.016 & $-0.050^{*}$ & $0.063 * *$ & $0.065^{* *}$ & $-0.061 * *$ & $0.074 *$ & $0.887 * *$ & $0.101 * *$ & $0.944 * *$ & $0.109 * *$ \\
\hline 68 & -0.012 & -0.030 & $0.046^{*}$ & 0.026 & 0.008 & -0.018 & $0.091 * *$ & 0.009 & 0.014 & $0.156^{* *}$ & $0.726 * *$ & $0.223 * *$ & $0.881 * *$ & $0.225^{* *}$ \\
\hline
\end{tabular}

Note. Negatively-worded items in italic; $* p<.05 . * * p<.01$ 
Table S3.

Parameter Estimates for the Measurement Part of the Population-Generating Model

\begin{tabular}{|c|c|c|c|c|c|c|}
\hline Items & $\begin{array}{l}\text { G-Factor } \\
\text { Loadings }\end{array}$ & $\begin{array}{l}\text { S-Factor } 1 \\
\text { Loadings }\end{array}$ & $\begin{array}{l}\text { S-Factor2 } \\
\text { Loadings }\end{array}$ & $\begin{array}{l}\text { S-Factor3 } \\
\text { Loadings }\end{array}$ & $\begin{array}{l}\text { Outcome } \\
\text { Loadings }\end{array}$ & Uniquenesses \\
\hline $\mathrm{X} 1$ & 0.450 & 0.650 & 0 & 0.150 & 0 & 0.353 \\
\hline $\mathrm{X} 2$ & 0.450 & 0.650 & 0.200 & 0 & 0 & 0.335 \\
\hline $\mathrm{X} 3$ & 0.600 & 0.600 & -0.100 & 0 & 0 & 0.270 \\
\hline $\mathrm{X} 4$ & 0.600 & 0.600 & 0 & -0.100 & 0 & 0.270 \\
\hline Y1 & 0.600 & 0 & 0.600 & 0.150 & 0 & 0.258 \\
\hline Y2 & 0.600 & 0.200 & 0.550 & 0 & 0 & 0.298 \\
\hline Y3 & 0.700 & -0.100 & 0.600 & 0 & 0 & 0.140 \\
\hline Y4 & 0.700 & 0 & 0.550 & -0.100 & 0 & 0.198 \\
\hline Z1 & 0.800 & 0.150 & 0 & 0.300 & 0 & 0.248 \\
\hline $\mathrm{Z} 2$ & 0.800 & 0 & 0.200 & 0.300 & 0 & 0.230 \\
\hline $\mathrm{Z3}$ & 0.700 & 0 & -0.100 & 0.500 & 0 & 0.250 \\
\hline Z4 & 0.700 & -0.100 & 0 & 0.500 & 0 & 0.250 \\
\hline$\overline{\mathrm{W} 1}$ & 0 & 0 & 0 & 0 & 0.500 & 0.750 \\
\hline W2 & 0 & 0 & 0 & 0 & 0.600 & 0.640 \\
\hline W3 & 0 & 0 & 0 & 0 & 0.700 & 0.510 \\
\hline W4 & 0 & 0 & 0 & 0 & 0.800 & 0.360 \\
\hline
\end{tabular}


Table S4.

Standardized Factor Loadings for the First-Order CFA and ESEM Solutions for the Simulated Data

\begin{tabular}{|c|c|c|c|c|c|c|}
\hline \multirow[b]{2}{*}{ Items } & \multicolumn{3}{|c|}{ ESEM } & \multirow[b]{2}{*}{ Uniquenesses } & \multicolumn{2}{|c|}{ ICM-CFA } \\
\hline & $\begin{array}{l}\text { Factor } 1 \\
\text { Loadings }\end{array}$ & $\begin{array}{l}\text { Factor } 2 \\
\text { Loadings }\end{array}$ & $\begin{array}{l}\text { Factor } 3 \\
\text { Loadings }\end{array}$ & & Factor Loadings & Uniquenesses \\
\hline $\mathrm{X} 1$ & $0.760 * *$ & $-0.127 * *$ & $0.114 * *$ & $0.412 * *$ & $0.752 * *$ & $0.434 * *$ \\
\hline $\mathrm{X} 2$ & $0.787 * *$ & $0.152 * *$ & $-0.130 * *$ & $0.358 * *$ & $0.782 * *$ & $0.388 * *$ \\
\hline $\mathrm{X} 3$ & $0.791 * *$ & $-0.070 * *$ & $0.149 * *$ & $0.289 * *$ & $0.843 * *$ & $0.289 * *$ \\
\hline $\mathrm{X} 4$ & $0.827 * *$ & $0.081 * *$ & -0.026 & $0.271 * *$ & $0.853 * *$ & $0.272 * *$ \\
\hline $\bar{Y} 1$ & $-0.048 * *$ & $0.766 * *$ & $0.157 * *$ & $0.278 * *$ & $0.852 * *$ & $0.273 * *$ \\
\hline Y2 & $0.194 * *$ & $0.758 * *$ & -0.027 & $0.279 * *$ & $0.834 * *$ & $0.304 * *$ \\
\hline Y3 & $-0.089 * *$ & $0.925 * *$ & $0.075 * *$ & $0.129 * *$ & $0.921 * *$ & $0.151 * *$ \\
\hline Y4 & $0.038 * *$ & $0.888 * *$ & -0.016 & $0.197 * *$ & $0.894 * *$ & $0.201 * *$ \\
\hline $\mathrm{Z1}$ & $0.224 * *$ & $0.063 * *$ & $0.698 * *$ & $0.227 * *$ & $0.875 * *$ & $0.234 * *$ \\
\hline $\mathrm{Z2}$ & 0.024 & $0.310 * *$ & $0.642 * *$ & $0.218 * *$ & $0.884 * *$ & $0.218 * *$ \\
\hline $\mathrm{Z3}$ & 0.013 & $-0.143 * *$ & $0.941 * *$ & $0.252 * *$ & $0.813 * *$ & $0.338 * *$ \\
\hline Z4 & $-0.129 * *$ & -0.009 & $0.935 * *$ & $0.245 * *$ & $0.820 * *$ & $0.328 * *$ \\
\hline
\end{tabular}




\section{Annotated Input Files Used in Study 2 (Simulated Data)}

\section{Title: Population Model - Input for the Data Generation}

! In all input files, statements preceded by! are annotations.

! The Monte Carlo facility is used to generate the data.

montecarlo:

names $=\mathrm{x} 1-\mathrm{x} 4 \mathrm{y} 1-\mathrm{y} 4 \mathrm{z} 1-\mathrm{z} 4 \mathrm{w} 1-\mathrm{w} 4 ;$ ! This statement indicates the variables.

ngroups $=2 ;$ ! This statement indicates the number of groups.

nobs $=800800 ; !$ This statement indicates the sample size in each group.

nreps $=1 ; !$ A single replication is requested.

save = B-ESEM.dat; ! This statement identifies the data set to be created .

! The following section defines the population model based on the parameters described in Table S3

! and Figure 2. The@ @ymbol precedes specific parameter values. Each input lines ends with;

! Factor loadings are noted with BY, regressions with ON, correlations with WITH, means and

! intercepts are noted between brackets []; variances and residuals are noted without brackets.

\section{model population:}

! All loadings invariant across groups

! Main loadings for the bifactor component ( $F G=G$-factor; FS1-FS3 = specific factors)

FG BY x1@.450; FG BY x2@.450; FG BYx3@.600; FG BY x4@.600;

FG BY y1@.600; FG BY y2@.600; FGBY y3@.700; FG BY y4@.700;

FG BY z1@.800; FG BY z2@.800; FG BY z3@.700; FG BY z4@.700;

FS1BYx1@.650; FS1BYx2@.650; FS1BYx3@.600; FS1 BYx4@.600;

FS2 BY y1@.600; FS2 BY y2@.550; FS2 BY y3@.600; FS2 BY y4@.550;

FS3 BY z1@.300; FS3 BYz2@.300; FS3 BYz3@.500; FS3 BY z4@.500;

Cross loadings

FS1BYy2@.200; FS1BY y3@-.100; FS1 BY z1@.150; FS1 BY z4@-.100;

FS2 BY X2@.200; FS2 BYX3@-.100; FS2 BYz2@.200; FS2 BYz3@-.100;

FS3 BY X1@.150; FS3 BY X4@-.100; FS3 BY y1@.150; FS3 BY y4@-.100;

! Outcome

OBYw1@.5; OBYw2@.6; OBYw3@.7; OBYw4@.8;

!Intercepts invariant across groups with intercept [Y2] non invariant across groups

[x1-x4@0]; [y1@0]; [y3-y4@0]; [z1-z4@0]; [w1-w4@0];

[y2@-.15];

!Uniquenesses invariant across groups

X1@0.353; X2@0.335; X3@0.270; X4@0.270; 
Y1@0.258; Y2@0.298; Y3@0.140; Y4@0.198;

Z1@0.248; Z2@0.230; Z3@0.250; Z4@0.250;

W1@0.750; W2@0.640;.W3@0.510; W4@0.360;

!Latent means all equal to 0 in group 1

[fG@0]; [fS1@0]; [fS2@0]; [fS3@0]; [O@0];

!Latent variances invariant across groups

fG@1; fS1@1; fS2@1; fS3@1; O@1;

!Latent covariances fixed at 0 for an orthogonal model (invariant across groups)

fG WITH fS1@0; fG WITH fS2@0; fG WITH fS3@0;

FS1WITHFS2@0; FS1 WITHFS3@0; FS2 WITHFS3@0;

! Latent regressions between the factors and the outcome (invariant across groups)

OONFG@.5; ONFS1@0; O ON FS2@0.5;OONFS3@0;

! Non invariant parameters in group 2

model population-g2:

! Latent means higher in group 2 on [FG] and [O] and lower on [FS1]

[fG@.5]; [fS1@-.5]; [O@.5];

lintercept [Y2] non invariant across groups

[y2@.15]; 
Title: ICM-CFA (Simulated Data)

! The following statement is used to identify the data file. Here, the data file is labelled BESEM.dat.

Data:

file $=$ B-ESEM.dat;

! The variables names function identifies all variables in the data set, in order of appearance.

! The usevar command identifies the variables used in the analysis.

Variable:

names $=\mathrm{x} 1-\mathrm{x} 4 \mathrm{y} 1-\mathrm{y} 4 \mathrm{z} 1-\mathrm{z} 4 \mathrm{w} 1-\mathrm{w} 4$ group;

usevar $=\mathrm{x} 1-\mathrm{x} 4 \mathrm{y} 1-\mathrm{y} 4 \mathrm{z} 1-\mathrm{z} 4$;

! The next section defines the analysis. Here Maximum Likelihood (ML) estimation is used.

Analysis:

ESTIMATOR $=\mathrm{ML}$

! The next section defines the model. An ICM-CFA model is specified

! with 3 factors (F1 to F3) defined respectively with items XI to X4, Y1 to Y4, and Z1 to Z4.

Model:

F1 BY X1-X4;

F2 BY Y1-Y4;

F3 BY Z1-Z4;

! Specific sections of output are requested.

Output: sampstat standardized SVALUES stdyx tech4; 
Title: Hierarchical CFA (Simulated Data)

! Previously presented sections of inputs are skipped to focus only on changes in the MODEL section.

! A first-order CFA model with no cross loading is specified with 3 factors (F1 to F3) defined

! respectively with items X1 to X4, Y1 to Y4, and Z1 to Z4.

! These 3 factors define a higher-order factor $\mathrm{HF}$.

Model:

F1 BY X1-X4;

F2 BY Y1-Y4;

F3 BY Z1-Z4;

HF BY F1-F3; 
Title: Bifactor CFA (Simulated Data)

! A bifactor CFA model is specified with 3 specific factors (FS1 to FS3)

! defined respectively with items X1 to X4, Y1 to Y4, and Z1 to Z4.

! All items are also used to define a global factor FG.

Model:

FG BY X1-Z4;

FS1 BY X1-X4;

FS2 BY Y1-Y4;

FS3 BY Z1-Z4;

! All factors are specified as orthogonal, with their correlations (WITH) constrained to be $0(@ 0)$.

fG WITH fS1@0;

fG WITH fS2@0

fGWITH fS3@0;

FS1 WITHFS2@0;

FS1 WITHFS3@0;

FS2 WITHFS3@0 
Title: First-order ESEM (Simulated Data)

! The next section defines the analysis. Here the Maximum Likelihood (ML) estimation is used

! together with target oblique rotation.

Analysis: ESTIMATOR = ML;

ROTATION = TARGET;

! An ESEM model is specified with target oblique rotation.

! The 3 factors (F1 to F3) are defined respectively with main loadings from items X1 to X4, Y1 to Y4,

! and Z1 to Z4. In addition to these main loadings, all other cross-loadings are estimated but targeted

! to be as close to 0 as possible ( 0). Factors forming a single set of ESEM factors (with cross-

! loadings between factors) are indicated by using the same label in parenthesis after * $(* 1)$.

Model:

F1 BY X1 X2 X3 X4 Y1 0 Y2 0 Y3 0 Y4 0 Z1 0 Z2 0 Z3 0 Z4 0 $(* 1)$;

F2 BY Y1 Y2 Y3 Y4 X1 0 X2 0 X3 0 X4 0 Z1 0 Z2 0 Z3 0 Z4 0 (*1);

F3 BY Z1 Z2 Z3 Z4 X1 0 X2 0 X3 0 X4 0 Y1 0 Y2 0 Y3 0 Y4 0 (*1); 
Title: Hierarchical ESEM using ESEM-Within-CFA (Simulated Data)

Analysis: ESTIMATOR $=$ ML;

! The previous ESEM model is re-expressed using CFA. No rotation is necessary.

! The model section uses the exact values of the non-standardized loadings and cross loadings

! estimated from the previous model as starts values (using *). For identification purposes, factor

! variances are constrained to be 1 (f1-f3@1;) and one item per factor has all cross loadings on non

! target factors constrained to be exactly equal to their ESEM values (using @).

! These 3 factors define a higher-order factor $\mathrm{HF}$.

Model:

f1 BY x $1 * 0.74674$; f1 BY x2*0.80372; f1 BY x3*0.80739; f1 BY x4*0.83759;

f1 BY y1*-0.05015; f1 BY y2*0.20610; f1 BY y3*-0.09183; f1 BY y4@0.03835;

f1 BY z1*0.22881; f1 BY z2*0.02457; f1 BY z3*0.01376; f1 BY z4@-0.13088;

f2 BY y1*0.79513; f2 BY y $2 * 0.80701$; f2 BY y $3 * 0.95053$; f2 BY y4*0.90008;

f2 BY x1*-0.12434; f2 BY x2*0.15514; f2 BY x3@-0.07168; f2 BY x4*0.08193;

f2 BY z1*0.06430; f2 BY z2*0.31927; f2 BY z3*-0.14645; f2 BY z4@-0.00922;

f3 BY z1*0.71349; f3 BY z2*0.66022; f3 BY z3*0.96202; f3 BY z4*0.95145;

f3 BY x1*0.11211; f3 BY x2*-0.13255; f3 BY x3@0.15235; f3 BY x4*-0.02669;

f3 BY y1*0.16258; f3 BY y2*-0.02858; f3 BY y3*0.07700; f3 BY y4@-0.01649;

f1-f3@1;

HF BY F1-F3; 
Title: Bifactor ESEM (Simulated Data)

! The next section defines the analysis. Here the Maximum Likelihood (ML) estimation is used

! together with orthogonal bifactor target rotation.

Analysis: ESTIMATOR = ML;

ROTATION = TARGET (orthogonal);

! The 3 specific factors (FS1 to FS3) are defined respectively with main loadings from items X1 to X4,

! Y1 to Y4, and Z1 to Z4. All other cross-loadings are estimated but targeted to be as close to 0 as

! possible ( 0). The global factor is defined through main loadings from all items, and is included in

! the same set of ESEM factors as FS1-FS3. Factors forming a single set of ESEM factors (with cross-

! loadings between factors) are indicated by using the same label in parenthesis after * (*1).

Model:

FG BY X1 X2 X3 X4 Y1 Y2 Y3 Y4 Z1 Z2 Z3 Z4 (*1);

FS1 BY X1 X2 X3 X4 Y1 0 Y2 0 Y3 0 Y4 0 Z1 0 Z2 0 Z3 0 Z4 0 (*1);

FS2 BY Y1 Y2 Y3 Y4 X1 0 X2 0 X3 0 X4 0 Z1 0 Z2 0 Z3 0 Z4 0 (*1);

FS3 BY Z1 Z2 Z3 Z4 X1 0 X2 0 X3 0 X4 0 Y1 0 Y2 0 Y3 0 Y4 0 (*1); 
Title: Multiple Group Configural Invariance (Simulated Data)

! The Grouping function identifies and labels the two groups

Variable:

names $=\mathrm{x} 1-\mathrm{x} 4 \mathrm{y} 1-\mathrm{y} 4 \mathrm{z} 1-\mathrm{z} 4 \mathrm{w} 1-\mathrm{w} 4$ group;

usevar $=\mathrm{x} 1-\mathrm{x} 4 \mathrm{y} 1-\mathrm{y} 4 \mathrm{z} 1-\mathrm{z} 4$;

grouping $=$ group $(1=\mathrm{G} 12=\mathrm{G} 2)$

! The next section defines the analysis. Here the Maximum Likelihood (ML) estimation is used

! together with orthogonal bifactor target rotation.

Analysis:

ESTIMATOR $=$ ML

ROTATION = TARGET (orthogonal);

! The model corresponds to the bifactor ESEM model presented previously: 3 specific

! factors (FS1 to FS3) are defined respectively with main loadings from items X1 to X4,

! Y1 to Y4, and Z1 to Z4. All other cross-loadings are estimated but targeted to be as close to 0 as

! possible ( 0). The global factor is defined through main loadings from all items, and is included in

! the same set of ESEM factors as FS1-FS3. Factors forming a single set of ESEM factors (with cross-

! loadings between factors) are indicated by using the same label in parenthesis after * $(* 1)$.

Model:

! Factor loadings and cross-loadings

FG BY X1 X2 X3 X4 Y1 Y2 Y3 Y4 Z1 Z2 Z3 Z4 (*1);

FS1 BY X1 X2 X3 X4 Y1 0 Y2 0 Y3 0 Y4 0 Z1 0 Z2 0 Z3 0 Z4 0 (*1);

FS2 BY Y1 Y2 Y3 Y4 X1 0 X2 0 X3 0 X4 0 Z1 0 Z2 0 Z3 0 Z4 0 (*1);

FS3 BY Z1 Z2 Z3 Z4 X1 0 X2 0 X3 0 X4 0 Y1 0 Y2 0 Y3 0 Y4 0 $(* 1)$;

! Items' intercepts

[x1-z4];

! Items' uniquenesses

$\mathrm{x} 1-\mathrm{z} 4$

! All latent means are constrained to be zero.

[FG@0FS1@0FS2@0FS3@0];

! In the next section, the parameters freely estimated in the second group are indicated.

MODEL G2:

! Factor loadings and cross-loadings are freely estimated in both groups

FG BY X1 X2 X3 X4 Y1 Y2 Y3 Y4 Z1 Z2 Z3 Z4 (*1);

FS1 BY X1 X2 X3 X4 Y1 0 Y2 0 Y3 0 Y4 0 Z1 0 Z2 0 Z3 0 Z4 0 (*1);

FS2 BY Y1 Y2 Y3 Y4 X1 0 X2 0 X3 0 X4 0 Z1 0 Z2 0 Z3 0 Z4 0 (*1); 
FS3 BY Z1 Z2 Z3 Z4 X1 0 X2 0 X3 0 X4 0 Y1 0 Y2 0 Y3 0 Y4 0 (*1);

! Items' intercepts are freely estimated in both groups

[x1-z4];

! Items' uniquenesses are freely estimated in both groups

$\mathrm{x} 1-\mathrm{z} 4$;

! All latent means are constrained to be zero in both groups

[FG@0FS1@0FS2@0FS3@0];

! By default, factor variances are constrained to 1 in all groups

! Specific sections of output are requested.

Output: sampstat standardized SVALUES stdyx tech4; 
Title: Multiple Group Weak (Loadings) Invariance (Simulated Data)

Model:

! Factor loadings and cross-loadings

FG BY X1 X2 X3 X4 Y1 Y2 Y3 Y4 Z1 Z2 Z3 Z4 (*1);

FS1 BY X1 X2 X3 X4 Y1 0 Y2 0 Y3 0 Y4 0 Z1 0 Z2 0 Z3 0 Z4 0 (*1);

FS2 BY Y1 Y2 Y3 Y4 X1 0 X2 0 X3 0 X4 0 Z1 0 Z2 0 Z3 0 Z4 0 (*1);

FS3 BY Z1 Z2 Z3 Z4 X1 0 X2 0 X3 0 X4 0 Y1 0 Y2 0 Y3 0 Y4 0 (*1);

! Items' intercepts

[x1-z4];

! Items' uniquenesses

$\mathrm{x} 1-\mathrm{z} 4$;

! All latent means are constrained to be zero.

[FG@0FS1@0FS2@0FS3@0];

! In the next section, the parameters freely estimated in the second group are indicated.

MODEL G2:

! Factor loadings and cross-loadings are specified as invariant across groups by default

! Items' intercepts are freely estimated in both groups

[x1-z4];

! Items' uniquenesses are freely estimated in both groups

$\mathrm{x} 1-\mathrm{z} 4$

! All latent means are constrained to be zero in both groups

[FG@0FS1@0FS2@0FS3@0];

! By default, factor variances will be freely estimated in the second group and constrained to be 1 in

! the first group 
Title: Multiple Group Strong (Loadings, Intercepts) Invariance (Simulated Data)

Model:

! Factor loadings and cross-loadings

FG BY X1 X2 X3 X4 Y1 Y2 Y3 Y4 Z1 Z2 Z3 Z4 (*1);

FS1 BY X1 X2 X3 X4 Y1 0 Y2 0 Y3 0 Y4 0 Z1 0 Z2 0 Z3 0 Z4 0 (*1);

FS2 BY Y1 Y2 Y3 Y4 X1 0 X2 0 X3 0 X4 0 Z1 0 Z2 0 Z3 0 Z4 0 (*1);

FS3 BY Z1 Z2 Z3 Z4 X1 0 X2 0 X3 0 X4 0 Y1 0 Y2 0 Y3 0 Y4 0 (*1);

! Items' intercepts

[x1-z4];

! Items' uniquenesses

$\mathrm{x} 1-\mathrm{z} 4$;

! All latent means are constrained to be zero.

[FG@0FS1@0FS2@0FS3@0];

! In the next section, the parameters freely estimated in the second group are indicated.

MODEL G2:

! Factor loadings, cross-loadings, and items' intercepts are invariant across groups by default

! Items' uniquenesses are freely estimated in both groups

$\mathrm{x} 1-\mathrm{z} 4$

! All latent means are freely estimated in the second group

[FG* $\mathrm{FS}^{*}$ FS2* FS3*];

! By default, factor variances will be freely estimated in the second group and constrained to be 1 in

! the first group 
Title: Multiple Group Partial Strong (Loadings, Partial Intercepts) Invariance (Simulated Data)

Model:

! Factor loadings and cross-loadings

FG BY X1 X2 X3 X4 Y1 Y2 Y3 Y4 Z1 Z2 Z3 Z4 (*1);

FS1 BY X1 X2 X3 X4 Y1 0 Y2 0 Y3 0 Y4 0 Z1 0 Z2 0 Z3 0 Z4 0 (*1);

FS2 BY Y1 Y2 Y3 Y4 X1 0 X2 0 X3 0 X4 0 Z1 0 Z2 0 Z3 0 Z4 0 (*1);

FS3 BY Z1 Z2 Z3 Z4 X1 0 X2 0 X3 0 X4 0 Y1 0 Y2 0 Y3 0 Y4 0 (*1);

! Items' intercepts

[x1-z4];

! Items' uniquenesses

$\mathrm{x} 1-\mathrm{z} 4$;

! All latent means are constrained to be zero.

[FG@0FS1@0FS2@0FS3@0];

! In the next section, the parameters freely estimated in the second group are indicated.

MODEL G2:

! Factor loadings, cross-loadings, and items' intercepts are invariant across groups by default

! Items' uniquenesses are freely estimated in both groups

$\mathrm{x} 1-\mathrm{z} 4$

! All latent means are freely estimated in the second group

[FG* $\mathrm{FS}^{*}$ FS2* FS3*];

! By default, factor variances will be freely estimated in the second group and constrained to be 1 in

! the first group

! Intercept [Y2] is freely estimated in the second group (partial invariance).

[y2]; 
Title: Multiple Group Strict (Loadings, Partial Intercepts, Uniquenesses) Invariance (Simulated Data)

Model:

! Factor loadings and cross-loadings

FG BY X1 X2 X3 X4 Y1 Y2 Y3 Y4 Z1 Z2 Z3 Z4 (*1);

FS1 BY X1 X2 X3 X4 Y1 0 Y2 0 Y3 0 Y4 0 Z1 0 Z2 0 Z3 0 Z4 0 (*1);

FS2 BY Y1 Y2 Y3 Y4 X1 0 X2 0 X3 0 X4 0 Z1 0 Z2 0 Z3 0 Z4 0 (*1);

FS3 BY Z1 Z2 Z3 Z4 X1 0 X2 0 X3 0 X4 0 Y1 0 Y2 0 Y3 0 Y4 0 (*1);

! Items' intercepts

[x1-z4];

! Items' uniquenesses: The parameter labels for the uniquenesses placed in parentheses (one label per

! uniqueness) indicate that the uniquenesses are to be constrained to invariance across groups.

$\mathrm{x} 1-\mathrm{z} 4(\mathrm{u} 1-\mathrm{u} 12)$

! All latent means are constrained to be zero.

[FG@0FS1@0FS2@0FS3@0];

! In the next section, the parameters freely estimated in the second group are indicated.

MODEL G2:

! Factor loadings, cross-loadings, and items' intercepts are invariant across groups by default

! Items' uniquenesses are invariant across groups due to labels used in the global model section.

! All latent means and intercept [Y2] are freely estimated in the second group

[FG* FS1* FS2* FS3*]; [y2];

! By default, factor variances will be freely estimated in the second group and constrained to be 1 in

! the first group 
Title: Multiple Group Variance-Covariance (Loadings, Partial Intercepts, Uniquenesses, Variances, Covariances) Invariance (Simulated Data) Model:

! Factor loadings and cross-loadings

FG BY X1 X2 X3 X4 Y1 Y2 Y3 Y4 Z1 Z2 Z3 Z4 (*1);

FS1 BY X1 X2 X3 X4 Y1 0 Y2 0 Y3 0 Y4 0 Z1 0 Z2 0 Z3 0 Z4 0 (*1);

FS2 BY Y1 Y2 Y3 Y4 X1 0 X2 0 X3 0 X4 0 Z1 0 Z2 0 Z3 0 Z4 0 (*1);

FS3 BY Z1 Z2 Z3 Z4 X1 0 X2 0 X3 0 X4 0 Y1 0 Y2 0 Y3 0 Y4 0 $(* 1)$;

! Items' intercepts

[x1-z4];

! Invariant uniquenesses

$\mathrm{x} 1-\mathrm{z} 4$ (u1-u12);

! All latent means are constrained to be zero.

[FG@0FS1@0FS2@0FS3@0];

! Even if the model is specified as orthogonal, equality constraints on the un-rotated factor

! covariances need to be indicated using parameters labels (in parentheses).

FG WITH FS1 (C1); FG WITH FS2 (C2); FG WITH FS3 (C3);

FS1 WITH FS2 (C4); FS1 WITH FS3 (C5); FS2 WITH FS3 (C6);

! In the next section, the parameters freely estimated in the second group are indicated.

MODEL G2:

! Factor loadings, cross-loadings, and items' intercepts are invariant across groups by default.

! Item' uniquenesses and factor covariances are invariant across groups due to labels used above.

! All latent means and intercept [Y2] are freely estimated in the second group

[FG* FS1* FS2* FS3*]; [y2];

! Factor variances constrained to be 1 (invariant) across groups

FG@1FS1@1FS2@1FS3@1; 
Title: Multiple Group Latent Means (Loadings, Partial Intercepts, Uniquenesses, Variances, Covariances, Latent Means) Invariance (Simulated Data)

Model:

! Factor loadings and cross-loadings

FG BY X1 X2 X3 X4 Y1 Y2 Y3 Y4 Z1 Z2 Z3 Z4 (*1);

FS1 BY X1 X2 X3 X4 Y1 0 Y2 0 Y3 0 Y4 0 Z1 0 Z2 0 Z3 0 Z4 0 (*1);

FS2 BY Y1 Y2 Y3 Y4 X1 0 X2 0 X3 $\sim 0$ X4 $\sim 0$ Z1 0 Z2 0 Z3 0 Z4 0 $(* 1)$;

FS3 BY Z1 Z2 Z3 Z4 X1 0 X2 0 X3 0 X4 0 Y1 0 Y2 0 Y3 0 Y4 0 (*1);

! Items' intercepts

[x1-z4];

! Invariant uniquenesses

! Invariant factor covariances

x1-z4 (u1-u12);

FG WITH FS1 (C1); FG WITH FS2 (C2); FG WITH FS3 (C3);

FS1 WITH FS2 (C4); FS1 WITH FS3 (C5); FS2 WITH FS3 (C6);

! All latent means are constrained to be zero.

[FG@0FS1@0FS2@0FS3@0];

! In the next section, the parameters freely estimated in the second group are indicated.

MODEL G2:

! Factor loadings, cross-loadings, and items' intercepts are invariant across groups by default.

! Item' uniquenesses and factor covariances are invariant across groups due to labels used above.

! Intercept [Y2] is freely estimated in the second group

! Latent means are constrained to be 0 (invariant) across groups.

[FG@0FS1@0FS2@0FS3@0]; [y2];

! Factor variances constrained to be 1 (invariant) across groups

FG@1FS1@1FS2@1FS3@1; 
Title: MIMIC (Null) Model (Simulated Data)

! The grouping variable is included in the list of variables to be used in the analysis.

Variable:

names $=\mathrm{x} 1-\mathrm{x} 4 \mathrm{y} 1-\mathrm{y} 4 \mathrm{z} 1-\mathrm{z} 4 \mathrm{w} 1-\mathrm{w} 4$ group;

usevar $=\mathrm{x} 1-\mathrm{x} 4 \mathrm{y} 1-\mathrm{y} 4 \mathrm{z} 1-\mathrm{z} 4$ group;

! The next section defines the analysis. Here the Maximum Likelihood (ML) estimation is used

! together with orthogonal bifactor target rotation.

Analysis:

ESTIMATOR $=$ ML;

ROTATION $=$ TARGET (orthogonal);

! The measurement model is defined as in the bifactor ESEM model presented previously.

Model:

! Factor loadings and cross-loadings

FG BY X1 X2 X3 X4 Y1 Y2 Y3 Y4 Z1 Z2 Z3 Z4 (*1);

FS1 BY X1 X2 X3 X4 Y1 0 Y2 0 Y3 0 Y4 0 Z1 0 Z2 0 Z3 0 Z4 0 (*1);

FS2 BY Y1 Y2 Y3 Y4 X1 0 X2 0 X3 0 X4 0 Z1 0 Z2 0 Z3 0 Z4 0 (*1);

FS3 BY Z1 Z2 Z3 Z4 X1 0 X2 0 X3 0 X4 0 Y1 0 Y2 0 Y3 0 Y4 0 (*1);

! In the MIMIC null model, the relations between the grouping variable and the items, as well as

! between the grouping variable and the factors, are constrained to be 0 .

X1-Z4 ON group@0;

FG FS1 FS2 FS3 ON group@0;

! Specific sections of output are requested.

Output: sampstat standardized SVALUES stdyx tech4; 
Title: MIMIC (Invariant) Model (Simulated Data)

Model:

FG BY X1 X2 X3 X4 Y1 Y2 Y3 Y4 Z1 Z2 Z3 Z4 (*1);

FS1 BY X1 X2 X3 X4 Y1 0 Y2 0 Y3 0 Y4 0 Z1 0 Z2 0 Z3 0 Z4 0 (*1);

FS2 BY Y1 Y2 Y3 Y4 X1 0 X2 0 X3 0 X4 0 Z1 0 Z2 0 Z3 0 Z4 0 (*1);

FS3 BY Z1 Z2 Z3 Z4 X1 0 X2 0 X3 0 X4 0 Y1 0 Y2 0 Y3 0 Y4 0 $(* 1)$;

! In the MIMIC invariant model, the relations between the grouping variable and the items are

! constrained to be 0 , while the relations between the grouping variable and the factors are

! freely estimated.

FG FS1 FS2 FS3 ON group;

X1-Z4 ON group@0; 
Title: MIMIC (Saturated) Model (Simulated Data)

Model:

FG BY X1 X2 X3 X4 Y1 Y2 Y3 Y4 Z1 Z2 Z3 Z4 (*1);

FS1 BY X1 X2 X3 X4 Y1 0 Y2 0 Y3 0 Y4 0 Z1 0 Z2 0 Z3 0 Z4 0 (*1);

FS2 BY Y1 Y2 Y3 Y4 X1 0 X2 0 X3 0 X4 0 Z1 0 Z2 0 Z3 0 Z4 0 (*1);

FS3 BY Z1 Z2 Z3 Z4 X1 0 X2 0 X3 0 X4 0 Y1 0 Y2 0 Y3 0 Y4 0 (*1);

! In the MIMIC saturated model, the relations between the grouping variable and the factors are

! constrained to be 0 , while the relations between the grouping variable and the items are

! freely estimated.

$\mathrm{X} 1-\mathrm{Z} 4 \mathrm{ON}$ group;

FG FS1 FS2 FS3 ON group@0; 
Title: MIMIC (Partial Invariance) Model (Simulated Data)

Model:

FG BY X1 X2 X3 X4 Y1 Y2 Y3 Y4 Z1 Z2 Z3 Z4 (*1);

FS1 BY X1 X2 X3 X4 Y1 0 Y2 0 Y3 0 Y4 0 Z1 0 Z2 0 Z3 0 Z4 0 (*1);

FS2 BY Y1 Y2 Y3 Y4 X1 0 X2 0 X3 0 X4 0 Z1 0 Z2 0 Z3 0 Z4 0 (*1);

FS3 BY Z1 Z2 Z3 Z4 X1 0 X2 0 X3 0 X4 0 Y1 0 Y2 0 Y3 0 Y4 0 (*1);

! In the final MIMIC model of partial invariance, the relations between the grouping variable and

$!$ most items are constrained to be 0 , while the relations between the grouping variable and the

! factors are freely estimated, as well as the relation between the grouping variable and the item with

! a non-invariant intercept.

X1-X4 ON group@0; Y1 ON group@0; Y3-Y4 ON group@0; Z1-Z4 ON group@0;

FG FS1 FS2 FS3 Y2 ON group; 


\section{Title: Predictive Model (Complete Mediation) (Simulated Data)}

! The grouping variable and the indicators of the outcome (W1-W4) are included in the list of

! variables to be used in the analysis.

Variable: names $=\mathrm{x} 1-\mathrm{x} 4 \mathrm{y} 1-\mathrm{y} 4 \mathrm{z} 1-\mathrm{z} 4 \mathrm{w} 1-\mathrm{w} 4$ group;

usevar $=\mathrm{x} 1-\mathrm{x} 4 \mathrm{y} 1-\mathrm{y} 4 \mathrm{zl}-\mathrm{z} 4 \mathrm{w} 1-\mathrm{w} 4$ group;

! The next section defines the analysis. Here the Maximum Likelihood (ML) estimation is used

! together with orthogonal bifactor target rotation.

Analysis:

ESTIMATOR $=$ ML; ROTATION $=$ TARGET (orthogonal);

! The measurement model is defined as in the bifactor ESEM model presented previously.

! One CFA factor (not part of the same set of ESEM factors) is added to the model (the outcome).

Model:

FG BY X1 X2 X3 X4 Y1 Y2 Y3 Y4 Z1 Z2 Z3 Z4 (*1);

FS1 BY X1 X2 X3 X4 Y1 0 Y2 0 Y3 0 Y4 0 Z1 0 Z2 0 Z3 0 Z4 0 (*1);

FS2 BY Y1 Y2 Y3 Y4 X1 0 X2 0 X3 0 X4 0 Z1 0 Z2 0 Z3 0 Z4 0 (*1);

FS3 BY Z1 Z2 Z3 Z4 X1 0 X2 0 X3 0 X4 0 Y1 0 Y2 0 Y3 0 Y4 0 (*1);

! The outcome factor

O BY W1-W4;

! The factors, as well as the non-invariant intercept identified in the MIMIC analysis, are regressed on

! the grouping variable.

FG FS1 FS2 FS3 y2 ON group;

! The outcome is regressed on the factors.

O ON FG FS1 FS2 FS3; 
Title: Predictive Model (Partial Mediation) (Simulated Data)

Model:

FG BY X1 X2 X3 X4 Y1 Y2 Y3 Y4 Z1 Z2 Z3 Z4 (*1);

FS1 BY X1 X2 X3 X4 Y1 0 Y2 0 Y3 0 Y4 0 Z1 0 Z2 0 Z3 0 Z4 0 (*1);

FS2 BY Y1 Y2 Y3 Y4 X1 0 X2 0 X3 $\sim 0$ X4 0 Z1 0 Z2 0 Z3 0 Z4 0 (*1);

FS3 BY Z1 Z2 Z3 Z4 X1 0 X2 0 X3 0 X4 0 Y1 0 Y2 0 Y3 0 Y4 0 (*1);

O BY W1-W4;

! The factors, as well as the non-invariant intercept identified in the MIMIC analysis, are regressed

! on the grouping variable. The outcome is also regressed on the grouping variable (partial

! mediation).

FG FS1 FS2 FS3 y2 O ON group;

! The outcome is regressed on the factors.

O ON FG FS1 FS2 FS3; 
Title: Predictive Model (Partial Mediation) Using Bootstrapping to Calculate Bias-Corrected Bootstrap Confidence Intervals (Simulated Data)

! The next section defines the analysis. Here Bootstrap estimation is used.

! This model is a Bifactor ESEM re-expressed through CFA. No rotation is necessary.

! The model section use, for all parameters, the exact values from the final predictive model for which

! bootstrap confidence intervals are required as starts values (using *).

! For identification purposes, factor variances are constrained to be 1 (f1-f3@1;) and one item per

! specific factor (FS1-FS3) as all cross loadings on non-target S-factors constrained to be exactly

! equal to their ESEM values (using @). No such constraint is required for the G-factor given that this

! model is specified as orthogonal.

Analysis:

BOOTSTRAP $=5000$;

Model:

fg BY x $1 * 0.47565$; fg BY x2*0.51619; fg BY x $3 * 0.69104$; fg BY x4*0.66507;

fg BY y $1 * 0.66688$; fg BY y $2 * 0.70089$; fg BY y $3 * 0.74618$; fg BY y $4 * 0.73479$;

fg BY z1*0.78988; fg BY z2*0.78830; fg BY z3*0.64542; fg BY z4*0.62698;

fs1 BY x $1 * 0.58619$; fs1 BY x $2 * 0.66138$; fs 1 BY x $3 * 0.51211$; fs1 BY x4*0.54301;

fs1 BY y1*-0.02798; fs1 BY y2*0.15757; fs1 BY y3*-0.13229; fs1 BY y4@-0.04785;

fs1 BY z1*0.12611; fs1 BY z2*-0.01304; fs1 BY z3@0.01261; fs1 BY z4*-0.07775;

fs2 BY y1*0.55084; fs2 BY y $2 * 0.50164$; fs2 BY y $3 * 0.54792$; fs2 BY y4*0.49895;

fs2 BY x $1 *-0.04398$; fs2 BY x2*0.16649; fs2 BY x3*-0.18205; fs2 BY x4@- 0.04842 ;

fs2 BY z1*-0.00855; fs2 BY z2*0.17398; fs2 BY z3@-0.08415; fs2 BY z4*0.02679;

fs3 BY z1*0.38016; fs3 BY z $2 * 0.36797$; fs3 BY z3*0.57256; fs3 BY z4*0.59676;

fs3 BY x1*0.11009; fs3 BY x2*-0.00721; fs3 BY x3*-0.01952; fs3 BY x4@-0.09399;

fs3 BY y1*0.13939; fs3 BY y2*-0.02089; fs3 BY y3*0.00124; fs3 BY y4@-0.07156;

o BYw1@1; o BYw2*1.20076; o BYw3*1.48388; o BY w4*1.69036;

fg ON group*0.45266; fs1 ON group*-0.50679;

fs2 ON group*0.03614; fs3 ON group*0.07745; o ON group*0.26972;

o ON fg*0.27947; o ON fs $1 *-0.01547$; o ON fs $2 * 0.20691$; o ON fs $3 *-0.06972$;

y2 ON group* 0.27648 ;

fs1 WITH fg@0.00000; fs2 WITH fg@0.00000; fs2 WITH fs1@0.00000;

fs3 WITH fg@0.00000; fs3 WITH fs1@0.00000; fs3 WITH fs2@0.00000;

$\mathrm{x} 1 * 0.37897 ; \mathrm{x} 2 * 0.30820 ; \mathrm{x} 3 * 0.26707 ; \mathrm{x} 4 * 0.27801$;

$\mathrm{y} 1 * 0.27790 ; \mathrm{y} 2 * 0.29478 ; \mathrm{y} 3 * 0.13632 ; \mathrm{y} 4 * 0.19727$

$\mathrm{z} 1 * 0.23483 ; \mathrm{z} 2 * 0.23179 ; \mathrm{z} 3 * 0.26629 ; \mathrm{z} 4 * 0.24572$;

w1*0.76143; w $2 * 0.63730$; w3*0.48113; w4*0.35837; 
fg@1; fs1@1; fs2@1; fs3@1; o*0.24669;

! The next section is used to request the estimation of indirect effects from the grouping variable to the

! outcome.

MODEL INDIRECT:

O IND Group;

OUTPUT:

! To request percentile-based symmetric confidence intervals, use "CINTERVAL"

! To request bootstrapped confidence intervals, use "CINTERVAL (boot)"

! To request bias-corrected bootstrapped confidence intervals, use "CINTERVAL (bcboot)"

SAMPSTAT STANDARDIZED RESIDUAL CINTERVAL (bcboot) MODINDICES (3.0) TECH2 TECH4; 
Title: Predictive Model Relaxing B-ESEM Limitation that all Factors from a Single Set Should Similarly Relate to Other Variables (Simulated Data) Analysis: ESTIMATOR = ML;

! This model is a Bifactor ESEM re-expressed through CFA. No rotation is necessary.

! The model section use, for all parameters, the exact values from a measurement model including all

! constructs as starts values (using *).

! For identification purposes, factor variances are constrained to be 1 (f1-f3@1;) and one item per

! specific factor (FS1-FS3) as all cross loadings on non-target S-factors constrained to be exactly

! equal to their ESEM values (using @). No such constraint is required for the G-factor given that this

$!$ model is specified as orthogonal.

! In this model the starts values for the factor correlations between the B-ESEM factors and other

! constructs are replaced by the required predictive paths.

Model:

fg BY $x 1 * 0.46458$; fg BY $x 2 * 0.47482$; fg BY $x 3 * 0.67622$; fg BY $\times 4 * 0.65699$;

fg BY y $1 * 0.68100$; fg BY y $2 * 0.74830$; fg BY y $3 * 0.75969 ;$ fg BY y $4 * 0.74731$

fg BY z1*0.80911; fg BY z2*0.80901; fg BY z3*0.67535; fg BY z4*0.65302;

fs 1 BY $x 1 * 0.58934$; fs1 BY x $2 * 0.71443$; fs 1 BY $\times 3 * 0.52628$; fs 1 BY $x 4 * 0.55292$;

fs1 BY y1*-0.01848; fs1 BY y2*0.12125; fs1 BY y3*-0.11663; fs1 BY y4@-0.03612;

fs1 BY z1*0.12633; fs1 BY z2*-0.00816; fs1 BY z3@0.00138; fs1 BY z4*-0.08033;

fs2 BY y1*0.55852; fs2 BY y $2 * 0.49354$; fs2 BY y $3 * 0.57152$; fs2 BY y4*0.51490;

fs2 BY x1*-0.06646; fs2 BY x $2 * 0.18023$; fs2 BY x $3 *-0.17586$; fs2 BY x4@-0.05946;

fs2 BY z1*-0.00509; fs2 BY z2*0.18630; fs2 BY z3@-0.08954; fs2 BY z4*0.03438;

fs3 BY z1*0.37411; fs3 BY z2*0.37026; fs3 BY z3*0.56066; fs3 BY z4*0.59805;

fs3 BY x1*0.09483; fs3 BY x2*0.01097; fs3 BY x3*-0.02009; fs3 BY x4@-0.10875;

fs3 BY y1*0.14318; fs3 BY y2*-0.03079; fs3 BY y3*0.01358; fs3 BY y4@-0.06608;

fs1 WITH fg@0.00000; fs2 WITH fg@0.00000; fs2 WITH fs1@0.00000; fs3 WITH fg@0.00000;

fs3 WITH fs1@0.00000; fs3 WITH fs2@0.00000;

fg@1; fs1@1; fs2@1; fs3@1;

$\mathrm{x} 1 * 0.38772 ; \mathrm{x} 2 * 0.27368 ; \mathrm{x} 3 * 0.27532 ; \mathrm{x} 4 * 0.27372 ; \mathrm{y} 1 * 0.27978 ; \mathrm{y} 2 * 0.31409 ; \mathrm{y} 3 * 0.13837$;

$\mathrm{y} 4 * 0.19837 ; \mathrm{z} 1 * 0.23468 ; \mathrm{z} 2 * 0.23232 ; \mathrm{z} 3 * 0.26619 ; \mathrm{z} 4 * 0.24426$;

! Outcome factor

o BYw1@1; o BY w2*1.20082; o BY w3*1.48391; o BY w4*1.69031; o*0.41394;

w1*0.76144; w2*0.63725; w3*0.48111; w4*0.35844;

! Requested predictive relations

FG FS1 y2 O ON group;

O ON FG FS2; 
Input Files Used in Study 1 (Real Data)

\section{Title: ICM-CFA Model of the SDQ-I (Real Data)}

data: file $=$ SDQ1.dat;

variable: names = Gender SDQ $1 \mathrm{SDQ} 2 \mathrm{SDQ} 3 \mathrm{SDQ} 4 \mathrm{SDQ} 5 \mathrm{SDQ} 6$

SDQ_7 SDQ_8 SDQ_9 SDQ_10 SDQ_11 SDQ_12 SDQ_13 SDQ_14 SDQ 15 SDQ_16

SDQ 17 SDQ 18 SDQ 19 SDQ 20 SDQ 21 SDQ 22 SDQ 23 SDQ 24 SDQ 25 SDQ 26

SDQ_27 SDQ_28 SDQ_29 SDQ_30 SDQ_31 SDQ_32 SDQ_33 SDQ_34 SDQ_35 SDQ_36

SDQ 37 SDQ 38 SDQ 39 SDQ 40 SDQ 41 SDQ 42 SDQ 43 SDQ 44 SDQ 45 SDQ 46

SDQ_47 SDQ_48 SDQ_49 SDQ_50 SDQ_51 SDQ_52 SDQ_53 SDQ_54 SDQ_55 SDQ_56

SDQ 57 SDQ 58 SDQ 59 SDQ 60 SDQ 61 SDQ 62 SDQ 63 SDQ 64 SDQ 65 SDQ 66

SDQ_67 SDQ_68 SDQ_69 SDQ_70 SDQ_71 SDQ_72 SDQ_73 SDQ_74 SDQ_75 SDQ_76;

missing = all (99);

usevar = SDQ 1 SDQ_2 SDQ 3 SDQ 4 SDQ 5 SDQ_6

SDQ 7 SDQ 8 SDQ 9 SDQ 10 SDQ 11 SDQ 12 SDQ_13 SDQ 14 SDQ 15 SDQ 16

SDQ 17 SDQ 18 SDQ 19 SDQ 20 SDQ 21 SDQ 22 SDQ 23 SDQ 24 SDQ 25 SDQ 26

SDQ_27 SDQ_28 SDQ_29 SDQ_30 SDQ_31 SDQ_32 SDQ_33 SDQ_34 SDQ_35 SDQ_36

SDQ 37 SDQ 38 SDQ 39 SDQ 40 SDQ 41 SDQ 42 SDQ 43 SDQ 44 SDQ 45 SDQ 46

SDQ_47 SDQ_48 SDQ_49 SDQ_50 SDQ_51 SDQ_52 SDQ_53 SDQ_54 SDQ_55 SDQ_56

SDQ 57 SDQ 58 SDQ 59 SDQ 60 SDQ 61 SDQ 62 SDQ 63 SDQ 64 SDQ 65 SDQ 66

SDQ_67 SDQ_68 SDQ_69 SDQ_70 SDQ_71 SDQ_72 SDQ_73 SDQ_74 SDQ_75 SDQ_76; Analysis: ESTIMATOR $=$ MLR;

Model:

esteem by SDQ 29 SDQ 45 SDQ 53 SDQ 67 SDQ 70 SDQ 72 SDQ 74

SDQ 76 SDQ 37 SDQ 61 ;

peer by SDQ 7 SDQ 14 SDQ 28 SDQ 36 SDQ 44 SDQ 52

SDQ_60 SDQ_69 SDQ_21;

appear by SDQ 1 SDQ 8 SDQ 15 SDQ 22 SDQ 38

SDQ_46 SDQ_54 SDQ_62 SDQ_30;

phy by SDQ 3 SDQ 10 SDQ 24 SDQ 32 SDQ 40 SDQ 48

SDQ 56 SDQ 64 SDQ 17 ;

parent by SDQ 5 SDQ_19 SDQ 26 SDQ 34 SDQ 42

SDQ 50 SDQ 58 SDQ 66 SDQ_12;

schocom by SDQ_2 SDQ_16 SDQ_31 SDQ_47 SDQ_63;

schoaff by SDQ 9 SDQ 23 SDQ 39 SDQ 55 SDQ 71 ;

Germcom by SDQ 4 SDQ_18 SDQ_33 SDQ_49 SDQ_73; 
Germaff by SDQ_11 SDQ_25 SDQ_41 SDQ 57 SDQ_65 ;

MathAff by SDQ 51 SDQ 35 SDQ 68 SDQ 20 SDQ 6;

MathCom by SDQ_27 SDQ_59 SDQ_13 SDQ_43 SDQ_75;

! Method Factor (negative items)

MF BY SDQ_30* SDQ_17 SDQ_12 SDQ_21 SDQ_47 SDQ_23 SDQ_33 SDQ_65

SDQ 75 SDQ 6 SDQ 37 SDQ 61; MF@1; [MF@0];

MF WITH esteem@0 peer@0 appear@0 phy@0 parent@0 schocom@0

schoaff@0 Germcom@0 Germaff@0 MathAff@0 MathCom@0;

! correlated uniquenesses between parallel worded items

SDQ 11 with SDQ 51 SDQ 71; SDQ 51 with SDQ 71;

SDQ_25 with SDQ_35 SDQ_39; SDQ_35 with SDQ_39;

SDQ 41 with SDQ_68 SDQ_9; SDQ_68 with SDQ 9;

SDQ_57 with SDQ_20 SDQ_55; SDQ_20 with SDQ_55;

SDQ_23 with SDQ_6 SDQ_65; SDQ_6 with SDQ_65;

SDQ 4 with SDQ 27 SDQ 16; SDQ_27 with SDQ_16;

SDQ_18 with SDQ 59 SDQ_2; SDQ_59 with SDQ 2;

SDQ 49 with SDQ 13 SDQ 63; SDQ 13 with SDQ 63;

SDQ 73 with SDQ 43 SDQ 31; SDQ 43 with SDQ 31;

SDQ 47 with SDQ 75 SDQ 33; SDQ 75 with SDQ 33;

output: sampstat standardized stdyx tech4; 
Title: Hierarchical CFA Model of the SDQ-I (Real Data)

! [...] Model section only

esteem by SDQ_29 SDQ_45 SDQ_53 SDQ_67 SDQ_70 SDQ_72 SDQ_74

SDQ 76 SDQ 37 SDQ 61;

peer by SDQ 7 SDQ_14 SDQ_28 SDQ_36 SDQ_44 SDQ_52

SDQ 60 SDQ 69 SDQ 21;

appear by SDQ_1 SDQ_8 SDQ_15 SDQ_22 SDQ_38

SDQ 46 SDQ 54 SDQ 62 SDQ 30 ;

phy by SDQ 3 SDQ 10 SDQ_24 SDQ_32 SDQ_40 SDQ 48

SDQ 56 SDQ 64 SDQ 17;

parent by SDQ_5 SDQ_19 SDQ_26 SDQ_34 SDQ_42

SDQ 50 SDQ 58 SDQ 66 SDQ 12;

schocom by SDQ_2 SDQ_16 SDQ_31 SDQ_47 SDQ_63;

schoaff by SDQ 9 SDQ 23 SDQ 39 SDQ 55 SDQ 71 ;

Germcom by SDQ_4 SDQ_18 SDQ_33 SDQ_49 SDQ_73 ;

Germaff by SDQ_11 SDQ 25 SDQ 41 SDQ 57 SDQ 65;

MathAff by SDQ 51 SDQ 35 SDQ 68 SDQ 20 SDQ 6 ;

MathCom by SDQ_27 SDQ 59 SDQ_13 SDQ_43 SDQ 75 ;

!Higher-Order factor

general by esteem peer appear phy parent schocom schoaff Germcom

Germaff MathAff MathCom:

MF BY SDQ_30* SDQ_17 SDQ_12 SDQ_21 SDQ_47 SDQ_23 SDQ_33 SDQ_65

SDQ 75 SDQ 6 SDQ 37 SDQ 61; MF@1; [MF@0];

MFWITH general@0 esteem@0 peer@0 appear@0 phy@0 parent@0 schocom@0

schoaff@0 Germcom@0 Germaff@0 MathAff@0 MathCom@0;

SDQ_11 with SDQ_51 SDQ_71; SDQ_51 with SDQ_71;

SDQ 25 with SDQ 35 SDQ 39 ; SDQ 35 with SDQ 39;

SDQ_41 with SDQ_68 SDQ_9; SDQ 68 with SDQ 99;

SDQ 57 with SDQ 20 SDQ 55; SDQ 20 with SDQ 55;

SDQ 23 with SDQ 6 SDQ 65 ; SDQ_6 with SDQ 65;

SDQ_4 with SDQ_27 SDQ 16; SDQ_27 with SDQ_16;

SDQ 18 with SDQ 59 SDQ 2 2; SDQ 59 with SDQ 2;

SDQ_49 with SDQ_13 SDQ_63; SDQ_13 with SDQ_63;

SDQ 73 with SDQ 43 SDQ 31; SDQ 43 with SDQ 31;

SDQ 47 with SDQ_75 SDQ 33; SDQ_75 with SDQ_33; 
Title: Bifactor CFA Model of the SDQ-I (Real Data)

! [...] Model section only

FG BY SDQ_1* SDQ_2 SDQ 3 SDQ_4 SDQ_5 SDQ_6 SDQ_7 SDQ 8 SDQ_9 SDQ_10

SDQ 11 SDQ 12 SDQ 13 SDQ 14 SDQ 15 SDQ 16 SDQ 17 SDQ 18 SDQ 19 SDQ 20

SDQ_21 SDQ_22 SDQ_23 SDQ_24 SDQ_25 SDQ_26 SDQ_27 SDQ_28 SDQ_29 SDQ_30

SDQ 31 SDQ 32 SDQ 33 SDQ 34 SDQ 35 SDQ 36 SDQ 37 SDQ 38 SDQ 39 SDQ 40

SDQ_41 SDQ_42 SDQ_43 SDQ_44 SDQ_45 SDQ_46 SDQ_47 SDQ_48 SDQ_49 SDQ_50

SDQ 51 SDQ 52 SDQ 53 SDQ 54 SDQ 55 SDQ 56 SDQ 57 SDQ 58 SDQ 59 SDQ 60

SDQ_61 SDQ_62 SDQ_63 SDQ_64 SDQ_65 SDQ_66 SDQ_67 SDQ_68 SDQ_69 SDQ_70

SDQ 71 SDQ 72 SDQ 73 SDQ 74 SDQ 75 SDQ 76 ;

fg@1;

FGWITH esteem@0 peer@0 appear@0 phy@0 parent@0

schocom@0 schoaff@0 Germcom@0 Germaff@0 mathcom@0 mathaff@0;

esteem WITH peer@0 appear@0 phy@0 parent@0

schocom@0 schoaff@0 Germcom@0 Germaff@0 mathcom@0 mathaff@0;

peerWITH appear@0 phy@0 parent@0

schocom@0 schoaff@0 Germcom@0 Germaff@0 mathcom@0 mathaff@0;

appearWITH phy@0 parent@0

schocom@0 schoaff@0 Germcom@0 Germaff@0 mathcom@0 mathaff@0;

phywith parent@0 schocom@0 schoaff@0 Germcom@0 Germaff@0 mathcom@0 mathaff@0;

parent with schocom@0 schoaff@0 Germcom@0 Germaff@0 mathcom@0 mathaff@0;

schocom WITH schoaff@0 Germcom@0 Germaff@0 mathcom@0 mathaff@0;

schoaff WITH Germcom@0 Germaff@0 mathcom@0 mathaff@0;

Germcom WITH Germaff@0 mathcom@0 mathaff@0;

GermafF WITH mathcom@0 mathaff@0; mathcom WITH mathaff@0;

esteem by SDQ_29 SDQ_45 SDQ_53 SDQ_67 SDQ_70 SDQ_72 SDQ_74

SDQ 76 SDQ 37 SDQ 61;

peer by SDQ 7 SDQ_14 SDQ_28 SDQ_36 SDQ_44 SDQ_52

SDQ 60 SDQ 69 SDQ 21;

appear by SDQ_1 SDQ_8 SDQ_15 SDQ_22 SDQ_38

SDQ 46 SDQ 54 SDQ 62 SDQ 30 ;

phy by SDQ 3 SDQ 10 SDQ_24 SDQ 32 SDQ 40 SDQ 48

SDQ 56 SDQ 64 SDQ 17

parent by SDQ 5 SDQ 19 SDQ 26 SDQ 34 SDQ 42

SDQ 50 SDQ 58 SDQ 66 SDQ 12 ; 
schocom by SDQ 2 SDQ 16 SDQ 31 SDQ 47 SDQ 63 ; schoaff by SDQ 9 SDQ 23 SDQ 39 SDQ 55 SDQ 71 ;

Germcom by SDQ 4 SDQ 18 SDQ 33 SDQ 49 SDQ 73 ;

Germaff by SDQ 11 SDQ 25 SDQ 41 SDQ 57 SDQ 65;

MathAff by SDQ_51 SDQ_35 SDQ_68 SDQ_20 SDQ 6;

MathCom by SDQ 27 SDQ 59 SDQ 13 SDQ 43 SDQ 75 ;

MF BY SDQ_30* SDQ_17 SDQ_12 SDQ_21 SDQ_47 SDQ_23 SDQ_33 SDQ_65

SDQ 75 SDQ 6 SDQ 37 SDQ 61; MF@1; [MF@0];

MFWITH FG@0 esteem@0 peer@0 appear@0 phy@0 parent@0 schocom@0

schoaff@0 Germcom@0 Germaff@0 MathAff@0 MathCom@0;

SDQ_11 with SDQ 51 SDQ 71; SDQ_51 with SDQ _71;

SDQ 25 with SDQ 35 SDQ 39; SDQ 35 with SDQ 39;

SDQ_41 with SDQ_68 SDQ_9; SDQ_68 with SDQ 99;

SDQ_57 with SDQ_20 SDQ_55; SDQ_20 with SDQ_55;

SDQ 23 with SDQ_6 SDQ_65; SDQ_6 with SDQ_65;

SDQ 4 with SDQ 27 SDQ 16; SDQ 27 with SDQ 16;

SDQ 18 with SDQ 59 SDQ 2; SDQ 59 with SDQ 2;

SDQ_49 with SDQ_13 SDQ_63; SDQ_13 with SDQ 63;

SDQ 73 with SDQ 43 SDQ 31; SDQ 43 with SDQ 31;

SDQ 47 with SDQ 75 SDQ 33 ; SDQ 75 with SDQ 33 ; 
Title: ESEM Model of the SDQ-I (Real Data)

! [...] Analysis and Model sections only

Analysis: ESTIMATOR = MLR; ROTATION = TARGET;

Model:

esteem by SDQ_1 0 SDQ_2 0 SDQ_3 0 SDQ 4 4 SDQ_5 0 SDQ_6 0 SDQ 7 0 SDQ_8 0 SDQ_9 0 SDQ_10 0 SDQ_11 0 SDQ_12 0 SDQ_13 0 SDQ 14 0 SDQ 15 0 SDQ 16 0 SDQ 17 0 SDQ 18 0 SDQ 19 0 SDQ 20 0 SDQ 21 0 SDQ 22 0 SDQ 23 0 SDQ 24 0 SDQ 25 0 SDQ 26 0 SDQ_27 0 SDQ_28 0 SDQ 29 SDQ 30 0 SDQ_31 $\sim 0$ SDQ_32 $\sim 0$ SDQ_33 0 SDQ 34 $\sim 0$ SDQ_35 0 SDQ_36 0 SDQ_37 SDQ 38 0 SDQ_39 0 SDQ 40 0 SDQ 41 0 SDQ 42 0 SDQ 43 0 SDQ 44 0 SDQ 45 SDQ 46 0 SDQ 47 0 SDQ 48 0 SDQ 49 0 SDQ 50 0 SDQ 51 0 SDQ 52 0 SDQ_53 SDQ_54 0 SDQ 55 $\sim 0$ SDQ_56 0 SDQ 57 $\sim 0$ SDQ_58 0 SDQ_59 0 SDQ_60 0 SDQ_61 SDQ_62 0 SDQ_63 0 SDQ_64 0 SDQ_65 0 SDQ 66 0 SDQ 67 SDQ 68 0 SDQ 69 0 SDQ 70 SDQ 71 0 SDQ 72 SDQ 73 0 SDQ 74 SDQ 75 0 SDQ $76(* 11)$;

peer by SDQ_1 0 SDQ 2 $\sim 0$ SDQ 3 0 SDQ 4 4 SDQ 5 0 SDQ 6 6 SDQ_7 SDQ 8 0 SDQ_9 0 SDQ_10 0 SDQ_11 0 SDQ 12 0 SDQ_13 0

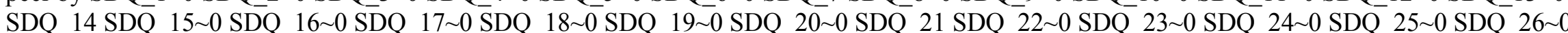
SDQ_27 0 SDQ_28 SDQ 29 0 SDQ_30 0 SDQ_31 0 SDQ_32 0 SDQ_33 0 SDQ_34 0 SDQ_35 0 SDQ_36 SDQ_37 0 SDQ_38 0 SDQ_39 0 SDQ 40 0 SDQ 41 0 SDQ 42 0 SDQ 43 0 SDQ 44 SDQ 45 0 SDQ 46 0 SDQ 47 0 SDQ 48 0 SDQ 49 0 SDQ 50 0 SDQ 51 0 SDQ 52 SDQ_53 0 SDQ_54 0 SDQ_55 0 SDQ_56 0 SDQ_57 0 SDQ 58 0 SDQ_59 0 SDQ_60 SDQ_61 0 SDQ_62 0 SDQ_63 0 SDQ_64 0 SDQ_65 0 SDQ $66 \sim 0$ SDQ 67 0 SDQ 68 0 SDQ 69 SDQ 70 0 SDQ 71 0 SDQ 72 0 SDQ 73 0 SDQ 74 0 SDQ $75 \sim 0$ SDQ $76 \sim 0(* t 1)$;

appear by SDQ 1 SDQ 2 0 SDQ 3 0 SDQ 4 0 SDQ 5 0 SDQ 6 0 SDQ 7 0 SDQ 8 SDQ 9 0 SDQ 10 0 SDQ 11 0 SDQ $12 \sim 0$ SDQ $13 \sim 0$ SDQ_14 0 SDQ 15 SDQ $16 \sim 0$ SDQ_17 0 SDQ_18 0 SDQ_19 0 SDQ_20 0 SDQ_21 0 SDQ_22 SDQ 23 0 SDQ 24 0 SDQ 25 0 SDQ 26 0 SDQ 27 0 SDQ 28 0 SDQ 29 0 SDQ 30 SDQ 31 0 SDQ 32 0 SDQ 33 0 SDQ 34 0 SDQ 35 0 SDQ 36 0 SDQ 37 0 SDQ 38 SDQ 39 0 SDQ_40 0 SDQ_41 0 SDQ_42 0 SDQ_43 0 SDQ 44 0 SDQ 45 0 SDQ_46 SDQ_47 0 SDQ_48 0 SDQ_49 0 SDQ_50 0 SDQ_51 0 SDQ_52 0 SDQ 53 0 SDQ 54 SDQ 55 0 SDQ 56 0 SDQ 57 0 SDQ 58 0 SDQ 59 0 SDQ 60 0 SDQ 61 0 SDQ 62 SDQ 63 0 SDQ 64 0 SDQ 65 0 SDQ 66 0 SDQ 67 0 SDQ 68 0 SDQ 69 0 SDQ 70 0 SDQ 71 0 SDQ 72 0 SDQ 73 0 SDQ 74 0 SDQ 75 0 SDQ 76 0 (*t1);

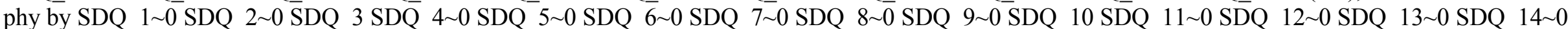
SDQ $15 \sim 0$ SDQ_16 0 SDQ_17 SDQ_18 0 SDQ 19 0 SDQ_20 0 SDQ_21 0 SDQ _22 0 SDQ_23 0 SDQ_24 SDQ_25 0 SDQ 26 0 SDQ 27 0 SDQ 28 0 SDQ 29 0 SDQ 30 0 SDQ 31 0 SDQ 32 SDQ 33 0 SDQ 34 0 SDQ 35 0 SDQ 36 0 SDQ 37 0 SDQ 38 0 SDQ 39 0 SDQ 40 SDQ_41 0 SDQ_42 0 SDQ_43 0 SDQ_44 0 SDQ_45 0 SDQ 46 0 SDQ_47 0 SDQ_48 SDQ_49 0 SDQ_50 0 SDQ_51 0 SDQ_52 0 SDQ_53 0 SDQ 54 0 SDQ 55 0 SDQ 56 SDQ 57 0 SDQ 58 0 SDQ 59 0 SDQ 60 0 SDQ 61 0 SDQ 62 0 SDQ 63 0 SDQ 64 SDQ $65 \sim 0$ SDQ $66 \sim 0$ SDQ_67 0 SDQ_68 0 SDQ_69 0 SDQ 70 0 SDQ 71 0 SDQ 72 0 SDQ_73 0 SDQ 74 0 SDQ_75 0 SDQ 76 0 $(* \bar{t} 1)$;

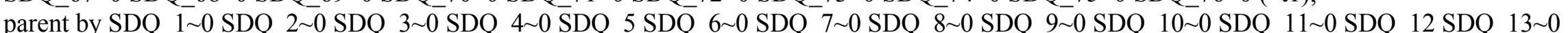
SDQ_14 0 SDQ_15 0 SDQ_16 0 SDQ_17 0 SDQ_18 0 SDQ_19 SDQ_20 0 SDQ_21 0 SDQ_22 0 SDQ_23 0 SDQ_24 0 SDQ_25 0 SDQ_26 SDQ 27 0 SDQ 28 0 SDQ 29 0 SDQ 30 0 SDQ 31 0 SDQ 32 0 SDQ 33 0 SDQ 34 SDQ 35 0 SDQ 36 0 SDQ 37 0 SDQ 38 0 SDQ 39 0 SDQ 40 0 SDQ 41 0 SDQ $42 \mathrm{SDQ} 43 \sim 0 \mathrm{SDQ} 44 \sim 0 \mathrm{SDQ}$ 45 0 SDQ 46 0 SDQ 47 $\sim 0$ SDQ 48 0 SDQ 49 0 SDQ 50 SDQ $51 \sim 0$ SDQ $52 \sim 0$ SDQ 53 0 SDQ 54 0 SDQ 55 0 SDQ 56 0 SDQ 57 0 SDQ 58 SDQ 59 0 SDQ 60 0 SDQ 61 0 SDQ $62 \sim 0$ SDQ $63 \sim 0$ SDQ 64 0 SDQ $65 \sim 0$ SDQ 66 SDQ $67 \sim 0$ SDQ $68 \sim 0$ SDQ 69 0 SDQ 70 0 SDQ 71 0 SDQ 72 0 SDQ 73 0 SDQ 74 0 SDQ 75 0 SDQ 76 0 $(* 11)$; schocom by SDQ_1 0 SDQ_2 SDQ_3 0 SDQ_4 0 SDQ_5 0 SDQ_6 0 SDQ_7 0 SDQ_8 0 SDQ_9 0 SDQ_10 0 SDQ_11 0 SDQ_12 0 SDQ_13 0 
SDQ_14 0 SDQ_15 0 SDQ_16 SDQ 17 0 SDQ_18 0 SDQ_19 0 SDQ_20 0 SDQ_21 0 SDQ_22 0 SDQ_23 0 SDQ_24 0 SDQ_25 0 SDQ_26 0 SDQ 27 0 SDQ 28 0 SDQ 29 0 SDQ 30 0 SDQ 31 SDQ 32 0 SDQ 33 0 SDQ 34 0 SDQ 35 0 SDQ 36 0 SDQ 37 0 SDQ 38 0 SDQ 39 0 SDQ_40 0 SDQ_41 0 SDQ_42 0 SDQ_43 0 SDQ_44 0 SDQ_45 0 SDQ_46 0 SDQ_47 SDQ_48 0 SDQ_49 0 SDQ_50 0 SDQ 51 0 SDQ 52 0 SDQ 53 0 SDQ 54 0 SDQ 55 0 SDQ 56 0 SDQ 57 0 SDQ 58 0 SDQ 59 0 SDQ 60 0 SDQ 61 0 SDQ 62 0 SDQ 63 SDQ 64 0 SDQ 65 0 SDQ_66 0 SDQ_67 0 SDQ_68 0 SDQ_69 0 SDQ_70 0 SDQ_71 0 SDQ_72 0 SDQ_73 0 SDQ_74 0 SDQ_75 0 SDQ 76 0 (*t1);

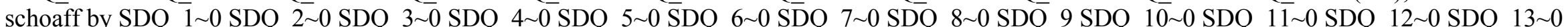
SDQ_14 0 SDQ_15 0 SDQ_16 0 SDQ_17 0 SDQ_18 0 SDQ_19 0 SDQ_20 0 SDQ_21 0 SDQ_22 0 SDQ_23 SDQ_24 0 SDQ_25 0 SDQ_26 0 SDQ 27 0 SDQ 28 0 SDQ 29 0 SDQ 30 0 SDQ 31 0 SDQ 32 0 SDQ 33 0 SDQ 34 0 SDQ 35 0 SDQ 36 0 SDQ 37 0 SDQ 38 0 SDQ 39 SDQ_40 0 SDQ_41 0 SDQ_42 0 SDQ_43 0 SDQ 44 0 SDQ_45 0 SDQ_46 0 SDQ_47 0 SDQ_48 0 SDQ_49 0 SDQ_50 0 SDQ_51 0 SDQ_52 0 SDQ 53 0 SDQ 54 0 SDQ 55 SDQ 56 0 SDQ 57 0 SDQ 58 0 SDQ 59 0 SDQ 60 0 SDQ 61 0 SDQ $62 \sim 0$ SDQ $63 \sim 0$ SDQ $64 \sim 0$ SDQ $65 \sim 0$ SDQ_66 0 SDQ 67 0 SDQ_68 0 SDQ_69 0 SDQ 70 0 SDQ 71 SDQ 72 0 SDQ 73 0 SDQ 74 0 SDQ_75 0 SDQ_76 0 (*t1);

Germcom by SDQ 1 0 SDQ_2 0 SDQ_3 0 SDQ 4 SDQ_5 0 SDQ_6 0 SDQ_7 0 SDQ_8 0 SDQ_9 0 SDQ_10 0 SDQ_11 0 SDQ 12 0 SDQ_13 0 SDQ_14 0 SDQ_15 0 SDQ_16 0 SDQ_17 0 SDQ_18 SDQ_19 0 SDQ_20 0 SDQ 21 0 SDQ_22 0 SDQ_23 0 SDQ_24 0 SDQ_25 0 SDQ_26 0 SDQ_27 0 SDQ_28 0 SDQ_29 0 SDQ_30 0 SDQ_31 0 SDQ_32 0 SDQ_33 SDQ_34 0 SDQ_35 0 SDQ_36 0 SDQ_37 0 SDQ_38 0 SDQ_39 0 SDQ 40 0 SDQ 41 0 SDQ 42 0 SDQ 43 0 SDQ 44 0 SDQ 45 0 SDQ 46 0 SDQ 47 0 SDQ 48 0 SDQ 49 SDQ 50 0 SDQ 51 0 SDQ 52 0 SDQ 53 0 SDQ 54 0 SDQ 55 0 SDQ 56 0 SDQ 57 0 SDQ 58 0 SDQ 59 0 SDQ 60 0 SDQ 61 0 SDQ 62 0 SDQ 63 0 SDQ 64 0 SDQ 65 0 SDQ 66 $\sim 0$ SDQ 67 0 SDQ 68 0 SDQ 69 0 SDQ 70 0 SDQ 71 0 SDQ 72 0 SDQ 73 SDQ 74 0 SDQ 75 0 SDQ 76 0 (*t1);

Germaff by SDQ_1 0 SDQ 2 2 0 SDQ 3 3 0 SDQ_4 0 SDQ 5 5 SDQ_6 0 SDQ 7 7 0 SDQ_8 0 SDQ_9 0 SDQ_10 0 SDQ_11 SDQ 12 0 SDQ_13 0 SDQ 14 0 SDQ 15 0 SDQ 16 0 SDQ 17 0 SDQ 18 0 SDQ 19 0 SDQ 20 0 SDQ 21 0 SDQ 22 0 SDQ 23 0 SDQ 24 0 SDQ 25 SDQ 26 0 SDQ_27 0 SDQ_28 0 SDQ_29 0 SDQ_30 0 SDQ 31 0 SDQ_32 0 SDQ 33 0 SDQ_34 0 SDQ_35 0 SDQ_36 0 SDQ_37 0 SDQ 38 0 SDQ_39 0 SDQ 40 0 SDQ 41 SDQ 42 0 SDQ 43 0 SDQ 44 0 SDQ 45 0 SDQ 46 0 SDQ 47 0 SDQ 48 0 SDQ 49 0 SDQ 50 0 SDQ 51 0 SDQ 52 0 SDQ_53 0 SDQ_54 0 SDQ_55 0 SDQ_56 0 SDQ_57 SDQ_58 0 SDQ_59 0 SDQ_60 0 SDQ_61 0 SDQ_62 0 SDQ_63 0 SDQ_64 0 SDQ_65 SDQ 66 0 SDQ 67 0 SDQ 68 0 SDQ 69 0 SDQ 70 0 SDQ 71 0 SDQ 72 0 SDQ 73 0 SDQ 74 0 SDQ 75 0 SDQ 76 0 (*t1); mathcom by SDQ_1 0 SDQ 2 2 0 SDQ 3 0 SDQ 4 0 SDQ 5 0 SDQ_6 0 SDQ 7 7 0 SDQ 8 0 SDQ $9 \sim 0$ SDQ 10 0 SDQ 11 0 SDQ_12 0 SDQ 13 SDQ 14 0 SDQ 15 0 SDQ 16 0 SDQ 17 0 SDQ 18 0 SDQ 19 0 SDQ 20 0 SDQ 21 0 SDQ 22 0 SDQ 23 0 SDQ 24 0 SDQ 25 0 SDQ 26 0 SDQ 27 SDQ 28 $\sim 0$ SDQ $29 \sim 0$ SDQ 30 0 SDQ 31 0 SDQ 32 $\sim 0$ SDQ_33 0 SDQ 34 $\sim 0$ SDQ 35 $\sim 0$ SDQ 36 $\sim 0$ SDQ 37 $\sim 0$ SDQ 38 0 SDQ 39 $\sim 0$

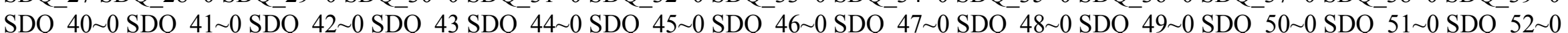
SDQ_53 0 SDQ_54 0 SDQ_55 0 SDQ_56 0 SDQ_57 0 SDQ_58 0 SDQ_59 SDQ_60 0 SDQ_61 0 SDQ_62 0 SDQ_63 0 SDQ_64 0 SDQ_65 0 SDQ 66 0 SDQ 67 0 SDQ 68 0 SDQ 69 0 SDQ 70 0 SDQ 71 0 SDQ 72 0 SDQ 73 0 SDQ 74 0 SDQ 75 SDQ 76 0 $(* 11)$

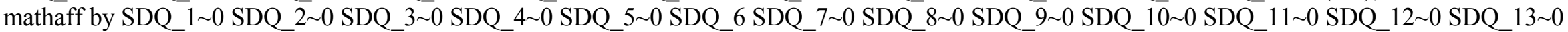
SDQ 14 0 SDQ 15 0 SDQ 16 0 SDQ 17 0 SDQ 18 0 SDQ 19 0 SDQ 20 SDQ 21 0 SDQ 22 0 SDQ 23 0 SDQ 24 0 SDQ 25 0 SDQ 26 0 SDQ 27 0 SDQ 28 0 SDQ 29 0 SDQ 30 0 SDQ 31 0 SDQ 32 0 SDQ 33 0 SDQ 34 0 SDQ 35 SDQ 36 0 SDQ 37 0 SDQ 38 0 SDQ 39 0 SDQ 40 0 SDQ 41 0 SDQ 42 0 SDQ 43 0 SDQ 44 0 SDQ 45 0 SDQ 46 0 SDQ 47 0 SDQ 48 0 SDQ 49 0 SDQ 50 0 SDQ 51 SDQ 52 0 SDQ 53 0 SDQ 54 0 SDQ 55 0 SDQ 56 0 SDQ 57 0 SDQ 58 0 SDQ 59 0 SDQ 60 0 SDQ 61 0 SDQ 62 0 SDQ 63 0 SDQ 64 0 SDQ 65 0 SDQ_66 0 SDQ_67 0 SDQ_68 SDQ_69 0 SDQ_70 0 SDQ_71 0 SDQ_72 0 SDQ_73 0 SDQ_74 0 SDQ_75 0 SDQ_76 0 (*t1); 
! Method Factor (negative items)

MF BY SDQ_30* SDQ_17 SDQ_12 SDQ_21 SDQ 47 SDQ_23 SDQ_33 SDQ_65

SDQ_75 SDQ_6 SDQ_37 SDQ_61; MF@1; [MF@0];

MFWITH esteem@0 peer@0 appear@0 phy@0 parent@0 schocom@0

schoaff@0 Germcom@0 Germaff@0 MathAff@0 MathCom@0;

! correlated uniquenesses between parallel worded items

SDQ_11 with SDQ_51 SDQ_71; SDQ_51 with SDQ_71;

SDQ 25 with SDQ 35 SDQ 39; SDQ 35 with SDQ 39;

SDQ_41 with SDQ_68 SDQ_9; SDQ_68 with SDQ_9;

SDQ 57 with SDQ 20 SDQ 55; SDQ 20 with SDQ 55;

SDQ_23 with SDQ_6 SDQ_65; SDQ_6 with SDQ 65;

SDQ 4 with SDQ_27 SDQ_16; SDQ_27 with SDQ_16;

SDQ_18 with SDQ_59 SDQ_2; SDQ_59 with SDQ_2;

SDQ_49 with SDQ_13 SDQ_63; SDQ_13 with SDQ_63;

SDQ_73 with SDQ_43 SDQ_31; SDQ_43 with SDQ_31;

SDQ_47 with SDQ_75 SDQ_33; SDQ_75 with SDQ_33; 
Title: Hierarchical ESEM Model of the SDQ-I Using ESEM-Within-CFA (Real Data) ! [...] Analysis and Model sections only

Analysis: ESTIMATOR = MLR;

Model:

esteem BY sdq_1*-0.03101; esteem BY sdq_2*0.01217; esteem BY sdq_3*-0.09151; esteem BY sdq_4@0.02081; esteem BY sdq_5*0.03906; esteem BY sdq_6*-0.04812; esteem BY sdq_7*-0.06562; esteem BY sdq $8 * 0.07463$; esteem BY sdq $9 * 0.03260$; esteem BY sdq $10 *-0.01524$; esteem BY sdq $11 *-0.05772$; esteem BY sdq $12 * 0.12921$; esteem BY sdq_13*-0.03180; esteem BY sdq_14*-0.07285; esteem BY sdq_15*-0.11861; esteem BY sdq 16@-0.02130; esteem BY sdq 17*0.02080; esteem BY sdq 18*-0.03832; esteem BY sdq_19*0.00802; esteem BY sdq_20*-0.00514; esteem BY sdq 21@-0.12155; esteem BY sdq_22@-0.08714; esteem BY sdq_23*0.09885; esteem BY sdq 24*0.01615; esteem BY sdq_25*-0.03904; esteem BY sdq_26*0.01240; esteem BY sdq_27@-0.03013; esteem BY sdq 28*-0.00719; esteem BY sdq 29*0.22094; esteem BY sdq $30 * 0.18339$; esteem BY sdq_31*0.11053; esteem BY sdq_32*0.07525; esteem BY sdq_33*0.08772; esteem BY sdq_34*-0.02310; esteem BY sdq 35*-0.05219; esteem BY sdq $36 * 0.14200$; esteem BY sdq_37*0.35244; esteem BY sdq_38*-0.03335; esteem BY sdq 39@0.10041; esteem BY sdq_40*-0.00166; esteem BY sdq 41*-0.01108; esteem BY sdq 42*-0.00535; esteem BY sdq $43 * 0.04976$; esteem BY sdq $44 * 0.08265$; esteem BY sdq $45^{*} 0.43268$; esteem BY sdq $46 * 0.17244$; esteem BY sdq $47 * 0.19713$; esteem BY sdq $48 * 0.00646$; esteem BY sdq_49*0.05793; esteem BY sdq_50@-0.05201; esteem BY sdq_51@0.03668; esteem BY sdq_52*0.02084; esteem BY sdq_53*0.50177; esteem BY sdq_54*0.07961; esteem BY sdq 55*0.09049; esteem BY sdq 56@0.01561; esteem BY sdq 57@0.01464; esteem BY sdq_58*-0.00679; esteem BY sdq 59*0.03239; esteem BY sdq 60*0.15877; esteem BY sdq $61 * 0.44200$; esteem BY sdq $62 * 0.34821$; esteem BY sdq $63 * 0.14271$; esteem BY sdq_64*-0.03137; esteem BY sdq $65 * 0.10076$; esteem BY sdq $66 * 0.01167$; esteem BY sdq $67 * 0.57164$; esteem BY sdq $68 *-0.01571$; esteem BY sdq $69 * 0.21330$; esteem BY sdq_70*0.42051; esteem BY sdq_71*0.09761; esteem BY sdq_72*0.64738; esteem BY sdq $73 * 0.17188$; esteem BY sdq $74 * 0.64337$; esteem BY sdq $75 * 0.09383$; esteem BY sdq $76 * 0.59995$;

peer BY sdq $1 * 0.01483$; peer BY sdq $2 * 0.00711$; peer BY sdq $3 * 0.02157$;

peer BY sdq_4@0.02496; peer BY sdq_5*0.00559; peer BY sdq_6*-0.04748;

peer BY sdq $7 * 0.60133$; peer BY sdq $8 *-0.01487$; peer BY sdq $9 * 0.00059$;

peer BY sdq_10*-0.01116; peer BY sdq_11*0.01508; peer BY sdq $12 * 0.03398$; peer BY sdq_13*0.05970; peer BY sdq_14*0.76648; peer BY sdq_15*0.03099; 
peer BY sdq_16@0.04426; peer BY sdq_17*0.04696; peer BY sdq 18*0.07575; peer BY sdq_19*-0.02550; peer BY sdq 20*-0.02445; peer BY sdq $21 * 0.89504$; peer BY sdq_22@0.00709; peer BY sdq 23*0.02470; peer BY sdq_24*0.05091; peer BY sdq 25*0.01956; peer BY sdq 26*0.00629; peer BY sdq 27@0.04108; peer BY sdq $28 * 0.50873$; peer BY sdq $29 * 0.06983$; peer BY sdq $30 *-0.05489$; peer BY sdq_31*0.00798; peer BY sdq $32 * 0.01557$; peer BY sdq_33*0.00357; peer BY sdq_34*0.10410; peer BY sdq_ $35 * 0.01648$; peer BY sdq_ $36 * 0.37885$; peer BY sdq $37 * 0.08214$; peer BY sdq $38 * 0.33493$; peer BY sdq 39@-0.00893; peer BY sdq_40*-0.01058; peer BY sdq $41 * 0.03624$; peer BY sdq $42 * 0.08547$; peer BY sdq $43 * 0.07033$; peer BY sdq $44 * 0.59996$; peer BY sdq $45 *-0.07896$; peer BY sdq 46*-0.00915; peer BY sdq $47 *-0.00916$; peer BY sdq $48 *-0.01087$; peer BY sdq_49*0.05702; peer BY sdq 50@-0.03239; peer BY sdq 51@-0.04187; peer BY sdq_52*0.64059; peer BY sdq_53*0.08961; peer BY sdq 54*0.06749; peer BY sdq_55*0.03405; peer BY sdq_56@-0.03591; peer BY sdq 57@0.00226; peer BY sdq_58*-0.03337; peer BY sdq_59*0.01515; peer BY sdq_60*0.57835; peer BY sdq $61 * 0.02987$; peer BY sdq $62 * 0.00648$; peer BY sdq $63 * 0.00454$; peer BY sdq_64*0.02051; peer BY sdq_65*-0.00968; peer BY sdq_66*0.02470; peer BY sdq $67 * 0.09766$; peer BY sdq $68 * 0.03386$; peer BY sdq $69 * 0.64351$; peer BY sdq_70*0.24271; peer BY sdq_71*0.02979; peer BY sdq_72@0.00827; peer BY sdq_73*0.03703; peer BY sdq $74 * 0.04893$; peer BY sdq_75*-0.01164; peer BY sdq $76 * 0.00385$;

appear BY sdq $1 * 0.71159$; appear BY sdq $2 * 0.09873$; appear BY sdq $3 * 0.13287$; appear BY sdq 4@0.06279; appear BY sdq 5*0.00368; appear BY sdq 6*-0.00785; appear BY sdq $7 *-0.04277$; appear BY sdq $8 * 0.70614$; appear BY sdq $\_* 0.11704$; appear BY sdq 10*-0.01854; appear BY sdq $11 * 0.11321$; appear BY sdq $12 *-0.03001$; appear BY sdq_13*0.02418; appear BY sdq_14*-0.00685; appear BY sdq_15*1.00287; appear BY sdq 16@0.07795; appear BY sdq 17*-0.12160; appear BY sdq 18*0.04556; appear BY sdq_19*0.01240; appear BY sdq_20*0.01631; appear BY sdq_21@-0.04602; appear BY sdq 22*1.02221; appear BY sdq 23*-0.00912; appear BY sdq 24*-0.10848; appear BY sdq 25*0.07942; appear BY sdq_26*0.03758; appear BY sdq_27@0.07097; appear BY sdq 28*-0.04027; appear BY sdq 29*0.05582; appear BY sdq $30 * 0.58827$; appear BY sdq $31 *-0.01727$; appear BY sdq $32 * 0.11016$; appear BY sdq $33 * 0.04432$; appear BY sdq 34*-0.00522; appear BY sdq $35 * 0.02454$; appear BY sdq $36 * 0.21725$; appear BY sdq 37*0.18567; appear BY sdq_38*0.48975; appear BY sdq_39@0.02326; appear BY sdq 40*0.00452; appear BY sdq $41 * 0.01634$; appear BY sdq $42 *-0.01316$; 
appear BY sdq 43*-0.02090; appear BY sdq 44*0.09312; appear BY sdq $45^{*} 0.23782$; appear BY sdq 46*0.46624; appear BY sdq 47*-0.00479; appear BY sdq 48*-0.00881; appear BY sdq_49*-0.01399; appear BY sdq_50@0.00336; appear BY sdq_51@0.00420; appear BY sdq $52 * 0.05729$; appear BY sdq $53 *-0.01538$; appear BY sdq $54 * 0.54715$; appear BY sdq_55*0.01001; appear BY sdq_56@0.05502; appear BY sdq_57@0.02400; appear BY sdq 58*0.00958; appear BY sdq 59*0.03606; appear BY sdq 60*0.07554; appear BY sdq $61 *-0.01190$; appear BY sdq $62 * 0.40915$; appear BY sdq $63 * 0.01776$; appear BY sdq $64 * 0.08782$; appear BY sdq $65 *-0.04775$; appear BY sdq $66 *-0.04915$; appear BY sdq 67*-0.04068; appear BY sdq 68*-0.03990; appear BY sdq $69 * 0.04566$; appear BY sdq 70*-0.00037; appear BY sdq 71*-0.02092; appear BY sdq 72@0.21957; appear BY sdq $73 *-0.04005$; appear BY sdq $74 * 0.00244$; appear BY sdq $\_75 * 0.01976$; appear BY sdq $76^{*}-0.01995$;

phy BY sdq $1 * 0.02194$; phy BY sdq $2 * 0.01452$; phy BY sdq_3*0.78947; phy BY sdq_4@-0.02107; phy BY sdq $5 * 0.01981$; phy BY sdq $6 * 0.01287$; phy BY sdq $\_7 * 0.03353$; phy BY sdq $8 * 0.06273$;

phy BY sdq $9 * 0.00662$; phy BY sdq $10 * 0.59057$; phy BY sdq $11 *-0.02425$;

phy BY sdq_12*-0.06819; phy BY sdq_13*-0.01218; phy BY sdq_14*0.00452;

phy BY sdq $15 *-0.03428$; phy BY sdq_16@0.03458; phy BY sdq $17 * 0.84509$; phy BY sdq $18 * 0.01133$; phy BY sdq $19 * 0.01286$; phy BY sdq $20^{*}-0.01088$; phy BY sdq 21@0.03890; phy BY sdq 22@-0.05882; phy BY sdq $23 *-0.00818$; phy BY sdq $24 * 0.96238$; phy BY sdq $25 * 0.00073$; phy BY sdq $26 *-0.00136$; phy BY sdq 27@-0.02262; phy BY sdq 28*0.04810; phy BY sdq 29*0.06779; phy BY sdq $30 *-0.01366$; phy BY sdq $31 * 0.04801$; phy BY sdq $32 * 0.44694$; phy BY sdq $33 * 0.00927$; phy BY sdq $34 *-0.00148$; phy BY sdq $35 * 0.03654$; phy BY sdq_36*-0.02582; phy BY sdq $37 *-0.05735$; phy BY sdq_ $38 * 0.02221$; phy BY sdq $39 @ 0.05285$; phy BY sdq $40 * 0.96105$; phy BY sdq $41 * 0.05432$; phy BY sdq $42 * 0.02684$; phy BY sdq $43 * 0.04910$; phy BY sdq $44 *-0.03567$; phy BY sdq $45^{*} 0.05679$; phy BY sdq $46 * 0.31726$; phy BY sdq $47 * 0.02376$; phy BY sdq_48*0.89070; phy BY sdq_49*0.00098; phy BY sdq_50@-0.01488; phy BY sdq $51 @ 0.03171$; phy BY sdq $52 * 0.00920$; phy BY sdq $53 * 0.06230$; phy BY sdq $54 * 0.07194$; phy BY sdq $55 * 0.02209$; phy BY sdq $56 * 1.07046$; phy BY sdq 57@0.01120; phy BY sdq 58*-0.02499; phy BY sdq 59*0.01162; phy BY sdq $60 * 0.03135$; phy BY sdq $61 *-0.03602$; phy BY sdq $62 *-0.02723$; phy BY sdq $63 * 0.01613$; phy BY sdq $64 * 0.72703$; phy BY sdq $65 *-0.00122$; phy BY sdq $66 * 0.04326$; phy BY sdq $67 * 0.04468$; phy BY sdq $68 * 0.06114$; phy BY sdq $69 * 0.01422$; phy BY sdq $\_70^{*}-0.04907$; phy BY sdq $71 * 0.03776$; 
phy BY sdq 72@0.02647; phy BY sdq $73 * 0.06399$; phy BY sdq $74 * 0.01812$; phy BY sdq $75 * 0.03129$; phy BY sdq $76 * 0.04231$;

parent BY sdq $1 *$ *0.02668; parent BY sdq $2 * 0.02864$; parent BY sdq $3 *-0.00981$; parent BY sdq 4@0.02773; parent BY sdq $5 * 0.57959$; parent BY sdq $6 * 0.07208$; parent BY sdq $7 * 0.03752$; parent BY sdq $8 * 0.00950$; parent BY sdq $9 * 0.11439$; parent BY sdq $10 * 0.05511$; parent BY sdq $11 * 0.03639$; parent BY sdq $12 * 0.39861$; parent BY sdq 13*-0.01614; parent BY sdq 14*-0.04690; parent BY sdq $15 * 0.00667$; parent BY sdq 16@0.08799; parent BY sdq 17*-0.03809; parent BY sdq 18*0.02067; parent BY sdq_19*0.38859; parent BY sdq_20*0.03514; parent BY sdq_21@0.01897; parent BY sdq 22@0.02610; parent BY sdq 23*0.07107; parent BY sdq 24*-0.04255; parent BY sdq 25*0.06513; parent BY sdq_26*0.38376; parent BY sdq_27@0.01378; parent BY sdq $28 * 0.07242$; parent BY sdq $29 * 0.02260$; parent BY sdq $30 * 0.06201$; parent BY sdq $31 * 0.05306$; parent BY sdq_32*-0.00869; parent BY sdq $33 * 0.03554$; parent BY sdq $34 * 0.73127$; parent BY sdq $35 * 0.01952$; parent BY sdq $36 *-0.01827$; parent BY sdq $37 * 0.11768$; parent BY sdq_38*-0.02072; parent BY sdq 39@0.01320; parent BY sdq $40 *-0.01890$; parent BY sdq $41 * 0.06423$; parent BY sdq $42 * 0.76171$; parent BY sdq $43 * 0.03504$; parent BY sdq $44 * 0.01979$; parent BY sdq $45 * 0.11303$; parent BY sdq $46 * 0.04475$; parent BY sdq $47 * 0.06342$; parent BY sdq $48 * 0.07274$; parent BY sdq 49*0.00470; parent BY sdq 50*0.86884; parent BY sdq 51@-0.02296; parent BY sdq $52 *-0.01037$; parent BY sdq $53 * 0.13723$; parent BY sdq $54 *-0.01702$; parent BY sdq 55*0.03126; parent BY sdq 56@-0.01330; parent BY sdq 57@0.06805; parent BY sdq $58 * 0.79620$; parent BY sdq $59 * 0.00180$; parent BY sdq $60 * 0.00609$; parent BY sdq $61 * 0.20753$; parent BY sdq $62 * 0.02179$; parent BY sdq $63 * 0.01376$; parent BY sdq $64 * 0.05815$; parent BY sdq $65 * 0.04031$; parent BY sdq $66 * 0.85028$; parent BY sdq $67 * 0.01947$; parent BY sdq $68 * 0.01100$; parent BY sdq $69 *-0.01439$; parent BY sdq $70 * 0.00436$; parent BY sdq $71 * 0.02078$; parent BY sdq $72 @-0.05797$; parent BY sdq $73 *-0.02819$; parent BY sdq $74 *-0.05795$; parent BY sdq $75 * 0.03648$; parent BY sdq $76^{*} 0.01392$

schocom BY sdq $1 *-0.03574$; schocom BY sdq $2 * 0.31972$; schocom BY sdq $3 * 0.06532$;

schocom BY sdq_4@0.19823; schocom BY sdq_5*-0.04472; schocom BY sdq_6*-0.09553; schocom BY sdq $7 *-0.19154$; schocom BY sdq $8 *-0.17366$; schocom BY sdq $9 * 0.17193$; schocom BY sdq_10*-0.10055; schocom BY sdq 11*-0.09512; schocom BY sdq_12*-0.00304; schocom BY sdq_13*0.05550; schocom BY sdq _ 14*-0.01751; schocom BY sdq_15*0.00149; schocom BY sdq 16*0.41965; schocom BY sdq 17*-0.17914; schocom BY sdq 18*0.12824; schocom BY sdq_19*-0.07013; schocom BY sdq_20*-0.03820; schocom BY sdq_21@-0.08778; 
schocom BY sdq_22@0.04052; schocom BY sdq 23*-0.08808; schocom BY sdq 24*-0.17587; schocom BY sdq_25*-0.07910; schocom BY sdq_26*-0.06565; schocom BY sdq_27@0.10586; schocom BY sdq_28*-0.09862; schocom BY sdq 29*0.06475; schocom BY sdq 30*-0.17817; schocom BY sdq $31 * 0.34433$; schocom BY sdq $32 * 0.14926$; schocom BY sdq $33 * 0.04007$; schocom BY sdq_34*0.09566; schocom BY sdq_35*-0.03328; schocom BY sdq_36*0.00935; schocom BY sdq_37*-0.12996; schocom BY sdq_38*0.14800; schocom BY sdq_39@0.26675; schocom BY sdq_40*-0.04308; schocom BY sdq 41*-0.03316; schocom BY sdq_42*0.15598; schocom BY sdq 43*0.09851; schocom BY sdq 44*0.05164; schocom BY sdq 45*-0.22275; schocom BY sdq 46*0.06105; schocom BY sdq 47*0.01216; schocom BY sdq 48*0.17752; schocom BY sdq 49*0.10702; schocom BY sdq 50@0.01514; schocom BY sdq 51@-0.07166; schocom BY sdq_52*0.11216; schocom BY sdq_53*-0.06347; schocom BY sdq_54*0.30532; schocom BY sdq 55*0.19671; schocom BY sdq 56@0.06650; schocom BY sdq 57@-0.03430; schocom BY sdq_58*0.00228; schocom BY sdq_59*0.07978; schocom BY sdq_60*0.18743; schocom BY sdq_61*-0.00385; schocom BY sdq 62*0.02041; schocom BY sdq 63*0.40308; schocom BY sdq_64*0.12476; schocom BY sdq_65*-0.08413; schocom BY sdq_66*0.10837; schocom BY sdq_67*0.11549; schocom BY sdq 68*-0.02362; schocom BY sdq 69*0.02854; schocom BY sdq $70 * 0.00562$; schocom BY sdq 71*0.24421; schocom BY sdq $72 @-0.01005$; schocom BY sdq_73*0.18943; schocom BY sdq $74 * 0.08310$; schocom BY sdq $75 * 0.02012$; schocom BY sdq $76 * 0.06152$;

schoaff BY sdq $1 * 0.01267$; schoaff BY sdq $2 * 0.30037$; schoaff BY sdq $3 *-0.06672$; schoaff BY sdq 4@-0.02909; schoaff BY sdq $5 * 0.02703$; schoaff BY sdq $6 * 0.01571$; schoaff BY sdq $7 * 0.09787$; schoaff BY sdq_ $8 * 0.06019$; schoaff BY sdq $9 * 0.57874$; schoaff BY sdq $10 * 0.09970$; schoaff BY sdq $11 * 0.17712$; schoaff BY sdq $12 * 0.02106$; schoaff BY sdq_13*-0.05071; schoaff BY sdq $14 * 0.04516$; schoaff BY sdq $15^{*} 0.02970$; schoaff BY sdq 16@0.35375; schoaff BY sdq 17*0.12652; schoaff BY sdq 18*-0.05048; schoaff BY sdq_19*0.00282; schoaff BY sdq_20*0.16064; schoaff BY sdq_21@0.02939; schoaff BY sdq 22@0.03393; schoaff BY sdq 23*0.20753; schoaff BY sdq 24*0.11611; schoaff BY sdq 25*0.19140; schoaff BY sdq 26*-0.02982; schoaff BY sdq 27@-0.00763; schoaff BY sdq $28 * 0.08785$; schoaff BY sdq $29 * 0.09841$; schoaff BY sdq $30 * 0.01285$; schoaff BY sdq $31 * 0.20521$; schoaff BY sdq $32 *-0.15592$; schoaff BY sdq $33 *-0.07493$; schoaff BY sdq $34 * 0.06088$; schoaff BY sdq $35^{*} 0.12109$; schoaff BY sdq $36 * 0.02556$; schoaff BY sdq $37 * 0.06750$; schoaff BY sdq $38 *-0.04896$; schoaff BY sdq $39 * 0.72990$; schoaff BY sdq 40*-0.01937; schoaff BY sdq 41*0.17576; schoaff BY sdq 42*-0.02253; schoaff BY sdq 43*-0.05860; schoaff BY sdq 44*-0.04273; schoaff BY sdq 45*0.09265; schoaff BY sdq 46*0.03906; schoaff BY sdq $47 * 0.00893$; schoaff BY sdq $48 *-0.12509$; 
schoaff BY sdq 49*-0.09122; schoaff BY sdq_50@-0.03307; schoaff BY sdq_51@0.08933; schoaff BY sdq $52 *-0.09556$; schoaff BY sdq $53 * 0.05800$; schoaff BY sdq $54 *-0.17392$; schoaff BY sdq_55*0.66120; schoaff BY sdq_56@-0.04085; schoaff BY sdq_57@0.20644; schoaff BY sdq $58 * 0.00145$; schoaff BY sdq $59 *-0.04844$; schoaff BY sdq $60 *-0.18589$; schoaff BY sdq $61 * 0.02385$; schoaff BY sdq $62 *-0.06655$; schoaff BY sdq $63 * 0.37975$; schoaff BY sdq $64 *-0.07081$; schoaff BY sdq $65 * 0.12569$; schoaff BY sdq $66 *-0.07108$; schoaff BY sdq $67 *-0.02117$; schoaff BY sdq $68 * 0.12000$; schoaff BY sdq $69 *-0.04491$; schoaff BY sdq $70 * 0.03310$; schoaff BY sdq 71*0.72868; schoaff BY sdq 72@0.00713; schoaff BY sdq $73 *-0.01735$; schoaff BY sdq $74 *-0.04215$; schoaff BY sdq $75 *-0.06356$; schoaff BY sdq 76*0.03296;

germcom BY sdq_1*0.02464; germcom BY sdq $2 * 0.31979$; germcom BY sdq $3 * 0.11665$; germcom BY sdq $4 * 0.88819$; germcom BY sdq $5 * 0.00246$; germcom BY sdq $6 * 0.06922$; germcom BY sdq_7*0.00814; germcom BY sdq $8 *-0.00219$; germcom BY sdq $9 *-0.01496$; germcom BY sdq_10*-0.02036; germcom BY sdq_11*0.34848; germcom BY sdq 12*0.13294; germcom BY sdq_13*-0.12882; germcom BY sdq_ $14 * 0.06945$; germcom BY sdq $15 * 0.02824$; germcom BY sdq_16@0.42804; germcom BY sdq_17*-0.03356; germcom BY sdq 18*0.84978; germcom BY sdq 19*-0.01960; germcom BY sdq 20*0.02158; germcom BY sdq 21@0.11222; germcom BY sdq_22@-0.02171; germcom BY sdq_23*0.10716; germcom BY sdq 24*-0.05364; germcom BY sdq 25*0.14376; germcom BY sdq 26*0.00068; germcom BY sdq 27@0.00548; germcom BY sdq $28 * 0.01925$; germcom BY sdq $29 *-0.03785$; germcom BY sdq_30*0.00515; germcom BY sdq $31 * 0.23068$; germcom BY sdq $32 *-0.15803$; germcom BY sdq $33 * 0.76849$; germcom BY sdq_34*-0.00141; germcom BY sdq_35*0.01786; germcom BY sdq_36*0.01015; germcom BY sdq 37*0.03312; germcom BY sdq 38*-0.01284; germcom BY sdq 39@-0.12649; germcom BY sdq 40*0.04597; germcom BY sdq 41*0.15598; germcom BY sdq 42*-0.07653; germcom BY sdq 43*-0.04550; germcom BY sdq 44*-0.02531; germcom BY sdq 45*-0.07370; germcom BY sdq_46*0.06314; germcom BY sdq_4 $7 * 0.24289$; germcom BY sdq $48 * 0.04927$; germcom BY sdq 49*0.67107; germcom BY sdq 50@-0.05134; germcom BY sdq 51@0.09341; germcom BY sdq_52*-0.12721; germcom BY sdq_53*-0.00815; germcom BY sdq_54*-0.12946; germcom BY sdq 55*-0.13366; germcom BY sdq 56@0.06107; germcom BY sdq 57@0.18819; germcom BY sdq_58*-0.04104; germcom BY sdq_59*-0.04233; germcom BY sdq_60*-0.01171; germcom BY sdq $61 * 0.20377$; germcom BY sdq $62 * 0.04860$; germcom BY sdq $63 * 0.13074$; germcom BY sdq_64*-0.16007; germcom BY sdq_65*0.28651; germcom BY sdq 66*-0.01054 germcom BY sdq_67*0.00605; germcom BY sdq 68*0.01225; germcom BY sdq 69*-0.02624; germcom BY sdq_70*-0.00307; germcom BY sdq_71*-0.16968; germcom BY sdq_72@-0.01015; germcom BY sdq_ $73 * 0.53200$; germcom BY sdq $74 * 0.04742$; germcom BY sdq $75 *-0.03063$; 
germcom BY sdq $76 *-0.03434$;

germaff BY sdq_ $1 *-0.03627$; germaff BY sdq $2 *-0.17220$; germaff BY sdq $3 *-0.03133$; germaff BY sdq_4@0.01653; germaff BY sdq_ $5 * 0.03043$; germaff BY sdq $6 *-0.14578$; germaff BY sdq $7 *-0.02423$; germaff BY sdq $8 *-0.01526$; germaff BY sdq $9 * 0.12656$; germaff BY sdq_10*0.03245; germaff BY sdq_11*0.77733; germaff BY sdq_12*-0.08203; germaff BY sdq $13 * 0.03138$; germaff BY sdq $14 *-0.00193$; germaff BY sdq $15 * 0.05813$; germaff BY sdq_16@-0.23242; germaff BY sdq_17*-0.07994; germaff BY sdq_18*0.24452; germaff BY sdq 19*0.02966; germaff BY sdq 20*-0.02903; germaff BY sdq 21@-0.10068; germaff BY sdq_22@0.04173; germaff BY sdq 23*0.14279; germaff BY sdq 24*0.00064; germaff BY sdq $25 * 0.82360$; germaff BY sdq 26*0.02056; germaff BY sdq 27@-0.00937; germaff BY sdq_28*-0.01456; germaff BY sdq_29*0.14622; germaff BY sdq_30*-0.02322; germaff BY sdq $31 *-0.01167$; germaff BY sdq $32 * 0.11916$; germaff BY sdq $33 * 0.21202$; germaff BY sdq_34*0.00199; germaff BY sdq $35 * 0.00157$; germaff BY sdq $36 *-0.04778$; germaff BY sdq_37*-0.13279; germaff BY sdq_38*0.02415; germaff BY sdq 39@0.23207; germaff BY sdq_40*-0.04781; germaff BY sdq_41*0.83719; germaff BY sdq $42 * 0.03849$; germaff BY sdq $43 * 0.03811$; germaff BY sdq $44 * 0.03801$; germaff BY sdq $45 *-0.00583$; germaff BY sdq 46*-0.02773; germaff BY sdq $47 * 0.01603$; germaff BY sdq $48 * 0.03247$; germaff BY sdq_49*0.37662; germaff BY sdq 50@0.05242; germaff BY sdq 51@-0.08716; germaff BY sdq $52 * 0.09654$; germaff BY sdq $53 *-0.00154$; germaff BY sdq $54 * 0.04087$; germaff BY sdq_55*0.28296; germaff BY sdq_56@-0.02630; germaff BY sdq $57 * 0.89432$; germaff BY sdq $58 *-0.01001$; germaff BY sdq $59 * 0.05214$; germaff BY sdq $60 * 0.01003$; germaff BY sdq_61*-0.16349; germaff BY sdq_62*0.02547; germaff BY sdq_63*-0.03054; germaff BY sdq $64 * 0.04280$; germaff BY sdq $65 * 0.78931$; germaff BY sdq $66^{*}-0.05294$; germaff BY sdq $67 * 0.01370$; germaff BY sdq_68*0.01890; germaff BY sdq 69*0.00657; germaff BY sdq 70*-0.00344; germaff BY sdq 71*0.27667; germaff BY sdq 72@-0.03087; germaff BY sdq_ $73 * 0.37518$; germaff BY sdq $\_74 * 0.03031$; germaff BY sdq $\_75 * 0.00124$; germaff BY sdq $76 * 0.03225$;

mathcom BY sdq $1 *-0.01355$; mathcom BY sdq_ $2 * 0.17807$; mathcom BY sdq $3 *-0.04975$; mathcom BY sdq 4@-0.12521; mathcom BY sdq $5 * 0.00470$; mathcom BY sdq 6*0.21715; mathcom BY sdq $7 * 0.06967$; mathcom BY sdq $8 * 0.04412$; mathcom BY sdq $99^{*}-0.08619$; mathcom BY sdq $10 * 0.00770$; mathcom BY sdq $11 *-0.02471$; mathcom BY sdq_12*0.13379; mathcom BY sdq $13 * 0.94431$; mathcom BY sdq $14 * 0.05180$; mathcom BY sdq_15*0.02195; mathcom BY sdq_16@0.17164; mathcom BY sdq_17*-0.01023; mathcom BY sdq_18*-0.00722; mathcom BY sdq 19*0.00664; mathcom BY sdq 20*0.12631; mathcom BY sdq 21@0.08614; mathcom BY sdq 22@0.03202; mathcom BY sdq 23*-0.02866; mathcom BY sdq 24*-0.01810; 
mathcom BY sdq $25 * 0.06313$; mathcom BY sdq $26 * 0.02995$; mathcom BY sdq $27 * 0.97555$; mathcom BY sdq $28 * 0.00675$; mathcom BY sdq $29 *-0.03438$; mathcom BY sdq $30 * 0.08342$; mathcom BY sdq $31 * 0.26082$; mathcom BY sdq $32 * 0.04376$; mathcom BY sdq_33*-0.00976; mathcom BY sdq $34 *-0.05170$; mathcom BY sdq $35 * 0.15805$; mathcom BY sdq 36*-0.03068; mathcom BY sdq_37*0.05779; mathcom BY sdq_38*-0.09554; mathcom BY sdq_39@-0.03657; mathcom BY sdq 40*-0.01202; mathcom BY sdq 41*0.01682; mathcom BY sdq 42*-0.02365; mathcom BY sdq $43 * 0.82870$; mathcom BY sdq_44*-0.05670; mathcom BY sdq_45*0.06601; mathcom BY sdq 46*-0.01572; mathcom BY sdq $47 * 0.16245$; mathcom BY sdq 48*0.02372; mathcom BY sdq_49*0.02387; mathcom BY sdq_50@0.03635; mathcom BY sdq_51@0.10636; mathcom BY sdq $52 * 0.06893$; mathcom BY sdq $53 * 0.00662$; mathcom BY sdq $54 * 0.03602$; mathcom BY sdq_55*-0.06226; mathcom BY sdq_56@-0.06294; mathcom BY sdq_57@0.01686; mathcom BY sdq $58 *-0.01569$; mathcom BY sdq $59 * 0.94183$; mathcom BY sdq $60 * 0.00518$; mathcom BY sdq $61 * 0.02473$; mathcom BY sdq $62 *-0.11578$; mathcom BY sdq_63*0.25556; mathcom BY sdq $64 * 0.11057$; mathcom BY sdq $65 * 0.04248$; mathcom BY sdq $66 *-0.13649$; mathcom BY sdq $67 * 0.08974$; mathcom BY sdq $68 * 0.20559$; mathcom BY sdq 69*-0.08096; mathcom BY sdq 70*-0.13314; mathcom BY sdq 71*0.09010; mathcom BY sdq 72@-0.09731; mathcom BY sdq $73 * 0.08917$; mathcom BY sdq $74 * 0.08093$; mathcom BY sdq $75 * 0.84844$; mathcom BY sdq $76 * 0.05491$;

mathaff BY sdq $1 * 0.02917$; mathaff BY sdq $2 *-0.04590$; mathaff BY sdq $3 * 0.03817$;

mathaff BY sdq 4@0.11083; mathaff BY sdq 5*-0.00099; mathaff BY sdq 6*1.10437; mathaff BY sdq $7 *-0.07729$; mathaff BY sdq $8 * 0.02207$; mathaff BY sdq $9 * 0.18071$;

mathaff BY sdq_10*-0.03058; mathaff BY sdq $11 *_{-} 0.02118$; mathaff BY sdq_12*-0.05929; mathaff BY sdq $13 * 0.17265$; mathaff BY sdq $14 *-0.05697$; mathaff BY sdq $15 *-0.03669$; mathaff BY sdq_16@-0.02219; mathaff BY sdq_17*-0.03774; mathaff BY sdq_18*0.02009; mathaff BY sdq 19*-0.01505; mathaff BY sdq 20*1.09659; mathaff BY sdq 21@-0.11460; mathaff BY sdq_22@-0.04446; mathaff BY sdq 23*0.20464; mathaff BY sdq 24*-0.03226; mathaff BY sdq 25*-0.03306; mathaff BY sdq 26*-0.03018; mathaff BY sdq 27@0.03160; mathaff BY sdq $28 * 0.00992$; mathaff BY sdq $29 * 0.03104$; mathaff BY sdq $30 *-0.00932$; mathaff BY sdq $31 *-0.04989$; mathaff BY sdq $32 * 0.02745$; mathaff BY sdq $33 * 0.07847$ mathaff BY sdq $34 * 0.00078$; mathaff BY sdq $35 * 1.06929$; mathaff BY sdq $36 * 0.02782$; mathaff BY sdq $37 *-0.05197$; mathaff BY sdq $38 * 0.05628$; mathaff BY sdq 39@0.17280; mathaff BY sdq $40 *-0.00423$; mathaff BY sdq $41 *-0.03239$; mathaff BY sdq $42 *-0.00995$; mathaff BY sdq $43 * 0.25704$; mathaff BY sdq $44 * 0.06873$; mathaff BY sdq $45 *-0.03605$; mathaff BY sdq $46 *-0.00531$; mathaff BY sdq $47 *-0.01081$; mathaff BY sdq $48 * 0.06323$; mathaff BY sdq 49*-0.00535; mathaff BY sdq 50@0.00584; mathaff BY sdq 51*1.26654; 
mathaff BY sdq $52 *-0.00616$; mathaff BY sdq $53 *-0.00407$; mathaff BY sdq $54 *-0.07068$; mathaff BY sdq_55*0.23184; mathaff BY sdq_56@0.00812; mathaff BY sdq_57@-0.00597; mathaff BY sdq $58 * 0.00621$; mathaff BY sdq $59 * 0.20240$; mathaff BY sdq $60 * 0.04015$; mathaff BY sdq $61 *-0.02459$; mathaff BY sdq $62 * 0.09024$; mathaff BY sdq $63 *-0.05669$; mathaff BY sdq $64 * 0.03170$; mathaff BY sdq $65 *-0.03723$; mathaff BY sdq $66 * 0.10015$; mathaff BY sdq $67 *-0.00482$; mathaff BY sdq $68 * 0.95882$; mathaff BY sdq $69 * 0.06475$; mathaff BY sdq $70 * 0.09384$; mathaff BY sdq $71 * 0.13859$; mathaff BY sdq $72 @ 0.06366$; mathaff BY sdq $73 *-0.09910$; mathaff BY sdq $74 *-0.05801$; mathaff BY sdq $75 * 0.18125$; mathaff BY sdq $76 *-0.04185$;

! Higher-order factor

general by esteem peer appear phy parent

schocom schoaff Germcom Germaff MathAff MathCom ;

! Method Factor (negative items)

MF BY SDQ 30* SDQ 17 SDQ 12 SDQ 21 SDQ 47 SDQ 23 SDQ 33 SDQ 65

SDQ 75 SDQ6 SDQ 37 SDQ 61; MF@1; [MF@0];

MFWITH general@0 esteem@0 peer@0 appear@0 phy@0 parent@0 schocom@0 schoaff@0 Germcom@0 Germaff@0 MathAff@0 MathCom@0;

! correlated uniquenesses between parallel worded items

SDQ 11 with SDQ 51 SDQ 71; SDQ 51 with SDQ 71;

SDQ_25 with SDQ_35 SDQ_39; SDQ_35 with SDQ_39;

SDQ 41 with SDQ 68 SDQ 9; SDQ 68 with SDQ 9;

SDQ_57 with SDQ_20 SDQ_55; SDQ_20 with SDQ_55;

SDQ 23 with SDQ 6 SDQ 65; SDQ 6 with SDQ 65;

SDQ_4 with SDQ_27 SDQ_16; SDQ_27 with SDQ_16;

SDQ 18 with SDQ 59 SDQ 2; SDQ 59 with SDQ 2;

SDQ_49 with SDQ_13 SDQ_63; SDQ_13 with SDQ_63;

SDQ 73 with SDQ 43 SDQ 31; SDQ 43 with SDQ 31;

SDQ_47 with SDQ_75 SDQ_33; SDQ_75 with SDQ_33; 
Title: Bifactor ESEM Model of the SDQ-I (Real Data)

! [...] Analysis and Model sections only

Analysis: ESTIMATOR = MLR; ROTATION = TARGET (orthogonal);

Model:

GLOBAL BY SDQ_1 SDQ_2 SDQ_3 SDQ 4 SDQ_5 SDQ_6 SDQ 7 SDQ_8 SDQ 9 SDQ_10

SDQ 11 SDQ 12 SDQ 13 SDQ 14 SDQ 15 SDQ 16 SDQ 17 SDQ 18 SDQ 19 SDQ 20

SDQ_21 SDQ_22 SDQ_23 SDQ_24 SDQ_25 SDQ_26 SDQ_27 SDQ_28 SDQ_29 SDQ_30

SDQ 31 SDQ 32 SDQ 33 SDQ 34 SDQ 35 SDQ 36 SDQ 37 SDQ 38 SDQ 39 SDQ 40

SDQ_41 SDQ_42 SDQ_43 SDQ_44 SDQ_45 SDQ_46 SDQ_47 SDQ_48 SDQ_49 SDQ_50

SDQ 51 SDQ 52 SDQ 53 SDQ 54 SDQ 55 SDQ 56 SDQ 57 SDQ 58 SDQ 59 SDQ 60

SDQ_61 SDQ_62 SDQ_63 SDQ_64 SDQ_65 SDQ_66 SDQ_67 SDQ_68 SDQ_69 SDQ_70

SDQ 71 SDQ 72 SDQ 73 SDQ 74 SDQ 75 SDQ $76(* t 1)$;

esteem by SDQ 1 0 SDQ 2 0 SDQ 3 0 SDQ 4 0 SDQ_5 0 SDQ_6 0 SDQ 7 0 SDQ_8 0 SDQ 9 0 SDQ_10 0 SDQ_11 0 SDQ_12 0 SDQ_13 0 SDQ 14 0 SDQ 15 0 SDQ 16 0 SDQ 17 0 SDQ 18 0 SDQ 19 0 SDQ 20 0 SDQ 21 0 SDQ 22 0 SDQ 23 0 SDQ 24 0 SDQ 25 0 SDQ 26 0 SDQ_27 0 SDQ_28 0 SDQ_29 SDQ_30 0 SDQ_31 0 SDQ_32 0 SDQ_33 0 SDQ_34 0 SDQ_35 0 SDQ_36 0 SDQ_37 SDQ_38 0 SDQ_39 0

SDQ 40 0 SDQ 41 0 SDQ 42 0 SDQ 43 0 SDQ 44 0 SDQ 45 SDQ 46 0 SDQ 47 0 SDQ 48 0 SDQ 49 0 SDQ 50 0 SDQ 51 0 SDQ 52 0

SDQ_53 SDQ_54 0 SDQ_55 0 SDQ_56 0 SDQ_57 0 SDQ_58 0 SDQ_59 0 SDQ_60 0 SDQ_61 SDQ_62 0 SDQ_63 0 SDQ_64 0 SDQ_65 0 SDQ 66 0 SDQ 67 SDQ 68 0 SDQ 69 0 SDQ 70 SDQ 71 0 SDQ 72 SDQ 73 0 SDQ 74 SDQ 75 0 SDQ $76(*$ t1);

peer by SDQ_1 0 SDQ_2 0 SDQ_3 0 SDQ 4 4 SDQ_5 0 SDQ 6 6 0 SDQ_7 SDQ 8 0 SDQ_9 0 SDQ_10 0 SDQ_11 0 SDQ_12 0 SDQ_13 0 SDQ_14 SDQ_15 0 SDQ 16 0 SDQ_17 0 SDQ_18 0 SDQ_19 0 SDQ_20 0 SDQ_21 SDQ_22 0 SDQ_23 0 SDQ_24 0 SDQ_25 0 SDQ_26 0 SDQ 27 0 SDQ 28 SDQ 29 0 SDQ 30 0 SDQ 31 0 SDQ 32 0 SDQ 33 0 SDQ 34 0 SDQ 35 0 SDQ 36 SDQ 37 0 SDQ 38 0 SDQ 39 0 SDQ_40 0 SDQ_41 0 SDQ_42 0 SDQ_43 0 SDQ_44 SDQ_45 0 SDQ_46 0 SDQ_47 0 SDQ_48 0 SDQ_49 0 SDQ_50 0 SDQ_51 0 SDQ_52 SDQ 53 0 SDQ 54 0 SDQ 55 0 SDQ 56 0 SDQ 57 0 SDQ 58 0 SDQ 59 0 SDQ 60 SDQ 61 0 SDQ 62 0 SDQ 63 0 SDQ 64 0 SDQ 65 0 SDQ_66 0 SDQ_67 0 SDQ_68 0 SDQ_69 SDQ_70 0 SDQ_71 0 SDQ_72 0 SDQ 73 $\sim 0$ SDQ_74 0 SDQ_75 0 SDQ_76 0 (*t1); appear by SDQ 1 SDQ 2 0 SDQ 3 0 SDQ 4 0 SDQ 5 0 SDQ 6 0 SDQ 7 0 SDQ 8 SDQ 9 0 SDQ 10 0 SDQ 11 0 SDQ $12 \sim 0$ SDQ $13 \sim 0$ SDQ_14 0 SDQ_15 SDQ 16 0 SDQ_17 0 SDQ_18 0 SDQ_19 0 SDQ 20 0 SDQ_21 0 SDQ_22 SDQ_23 0 SDQ_24 0 SDQ 25 0 SDQ_26 0 SDQ 27 0 SDQ 28 0 SDQ 29 0 SDQ 30 SDQ 31 0 SDQ 32 0 SDQ 33 0 SDQ 34 0 SDQ 35 0 SDQ 36 0 SDQ 37 0 SDQ 38 SDQ 39 0 SDQ_40 0 SDQ_41 0 SDQ_42 0 SDQ_43 0 SDQ_44 0 SDQ 45 0 SDQ_46 SDQ_47 0 SDQ_48 0 SDQ_49 0 SDQ_50 0 SDQ_51 0 SDQ_52 0 SDQ 53 0 SDQ 54 SDQ 55 0 SDQ 56 0 SDQ 57 0 SDQ 58 0 SDQ 59 0 SDQ 60 0 SDQ 61 0 SDQ 62 SDQ $63 \sim 0$ SDQ $64 \sim 0$ SDQ $65 \sim 0$ SDQ_66 0 SDQ 67 0 SDQ_68 0 SDQ 69 0 SDQ_70 0 SDQ_71 0 SDQ_72 0 SDQ_73 0 SDQ_74 0 SDQ_75 0 SDQ_76 0 (*t1);

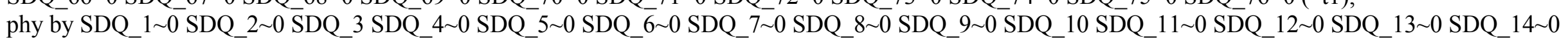
SDQ 15 0 SDQ_16 0 SDQ_17 SDQ_18 0 SDQ 19 0 SDQ_20 0 SDQ_21 0 SDQ 22 0 SDQ_23 0 SDQ_24 SDQ_25 0 SDQ 26 0 SDQ_27 0 SDQ 28 0 SDQ 29 0 SDQ 30 0 SDQ 31 0 SDQ 32 SDQ 33 0 SDQ 34 0 SDQ 35 0 SDQ 36 0 SDQ 37 0 SDQ 38 0 SDQ 39 0 SDQ 40 SDQ 41 0 SDQ 42 0 SDQ 43 0 SDQ 44 0 SDQ 45 0 SDQ 46 0 SDQ 47 0 SDQ 48 SDQ 49 0 SDQ 50 0 SDQ 51 0 SDQ 52 0 SDQ 53 0 SDQ_54 0 SDQ_55 0 SDQ_56 SDQ_57 0 SDQ_58 0 SDQ_59 0 SDQ_60 0 SDQ_61 0 SDQ_62 0 SDQ_63 0 SDQ_64 SDQ_65 0 SDQ_66 0 
SDQ _67 0 SDQ 68 0 SDQ 69 0 SDQ 70 0 SDQ 71 0 SDQ 72 0 SDQ 73 0 SDQ 74 0 SDQ 75 0 SDQ 76 0 (*t1);

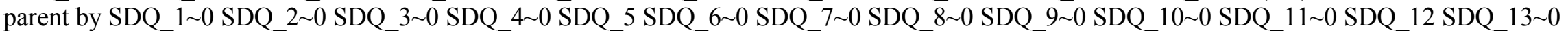
SDQ_14 0 SDQ_15 0 SDQ_16 0 SDQ_17 0 SDQ_18 0 SDQ_19 SDQ_20 0 SDQ_21 0 SDQ_22 0 SDQ_23 0 SDQ_24 0 SDQ_25 0 SDQ_26 SDQ 27 0 SDQ 28 0 SDQ 29 0 SDQ 30 0 SDQ 31 0 SDQ 32 0 SDQ 33 0 SDQ 34 SDQ 35 0 SDQ 36 0 SDQ 37 0 SDQ 38 0 SDQ 39 0 SDQ_40 0 SDQ_41 0 SDQ_42 SDQ_43 0 SDQ_44 0 SDQ_45 0 SDQ_46 0 SDQ_47 0 SDQ_48 0 SDQ_49 0 SDQ_50 SDQ_51 0 SDQ_52 0 SDQ 53 0 SDQ 54 0 SDQ 55 0 SDQ 56 0 SDQ 57 0 SDQ 58 SDQ 59 0 SDQ 60 0 SDQ 61 0 SDQ 62 0 SDQ 63 0 SDQ 64 0 SDQ 65 0 SDQ_66 SDQ_67 0 SDQ_68 0 SDQ_69 0 SDQ_70 0 SDQ 71 $\sim 0$ SDQ_72 0 SDQ_73 0 SDQ_74 0 SDQ_75 0 SDQ 76 0 $(* 11)$;

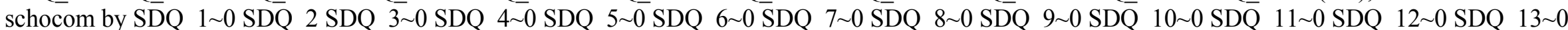
SDQ 14 0 SDQ 15 0 SDQ_16 SDQ 17 0 SDQ_18 0 SDQ 19 0 SDQ 20 0 SDQ 21 $\sim 0$ SDQ 22 0 SDQ 23 0 SDQ 24 0 SDQ 25 0 SDQ 26 0 SDQ 27 0 SDQ 28 0 SDQ 29 0 SDQ 30 0 SDQ 31 SDQ 32 0 SDQ 33 0 SDQ 34 0 SDQ 35 0 SDQ 36 0 SDQ 37 0 SDQ 38 0 SDQ 39 0 SDQ_40 0 SDQ_41 0 SDQ_42 0 SDQ_43 0 SDQ_44 0 SDQ 45 0 SDQ_46 0 SDQ 47 SDQ_48 0 SDQ_49 0 SDQ_50 0 SDQ_51 0 SDQ 52 0 SDQ_53 0 SDQ_54 0 SDQ_55 0 SDQ_56 0 SDQ_57 0 SDQ_58 0 SDQ_59 0 SDQ_60 0 SDQ_61 0 SDQ_62 0 SDQ_63 SDQ_64 0 SDQ_65 0 SDQ_66 0 SDQ_67 0 SDQ_68 0 SDQ 69 0 SDQ_70 0 SDQ_71 0 SDQ_72 0 SDQ_73 0 SDQ 74 0 SDQ_75 0 SDQ_76 0 (*t1);

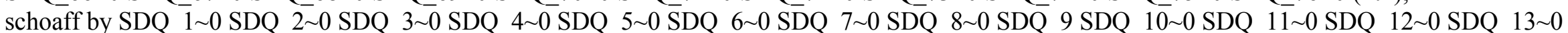
SDQ 14 0 SDQ 15 0 SDQ 16 0 SDQ 17 0 SDQ 18 0 SDQ 19 0 SDQ 20 0 SDQ 21 0 SDQ 22 0 SDQ 23 SDQ 24 0 SDQ 25 0 SDQ 26 0 SDQ_27 0 SDQ_28 0 SDQ_29 0 SDQ_30 0 SDQ_31 0 SDQ_32 0 SDQ_33 0 SDQ_34 0 SDQ_35 0 SDQ_36 0 SDQ_37 0 SDQ_38 0 SDQ_39 SDQ 40 0 SDQ 41 0 SDQ 42 0 SDQ 43 0 SDQ 44 0 SDQ 45 0 SDQ 46 0 SDQ 47 0 SDQ 48 0 SDQ 49 0 SDQ 50 0 SDQ 51 0 SDQ 52 0 SDQ_53 0 SDQ_54 0 SDQ_55 SDQ_56 0 SDQ_57 0 SDQ_58 0 SDQ_59 0 SDQ_60 0 SDQ_61 0 SDQ_62 0 SDQ_63 0 SDQ_64 0 SDQ_65 0 SDQ 66 0 SDQ 67 0 SDQ 68 0 SDQ 69 0 SDQ 70 0 SDQ 71 SDQ 72 0 SDQ 73 0 SDQ 74 0 SDQ 75 0 SDQ 76 0 (*t1);

Germcom by SDQ 1 0 SDQ_2 0 SDQ_3 0 SDQ 4 SDQ_5 0 SDQ_6 0 SDQ_7 0 SDQ_8 0 SDQ_9 0 SDQ_10 0 SDQ_11 0 SDQ 12 0 SDQ_13 0 SDQ 14 0 SDQ 15 0 SDQ 16 0 SDQ 17 0 SDQ 18 SDQ 19 0 SDQ 20 0 SDQ 21 0 SDQ 22 0 SDQ 23 0 SDQ 24 0 SDQ 25 0 SDQ 26 0 SDQ_27 0 SDQ_28 0 SDQ_29 0 SDQ_30 0 SDQ_31 0 SDQ_32 0 SDQ_33 SDQ_34 0 SDQ_35 0 SDQ 36 0 SDQ_37 0 SDQ 38 0 SDQ 39 0

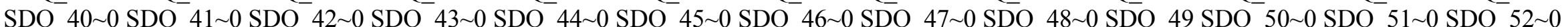
SDQ_53 0 SDQ_54 0 SDQ_55 0 SDQ_56 0 SDQ_57 0 SDQ_58 0 SDQ_59 0 SDQ_60 0 SDQ_61 0 SDQ_62 0 SDQ_63 0 SDQ_64 0 SDQ_65 0 SDQ 66 0 SDQ 67 0 SDQ 68 0 SDQ 69 0 SDQ 70 0 SDQ 71 0 SDQ 72 0 SDQ 73 SDQ 74 0 SDQ 75 0 SDQ 76 0 (*t1);

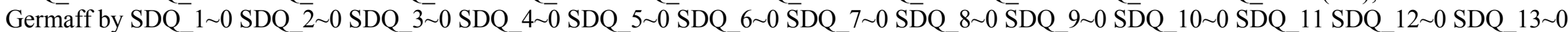

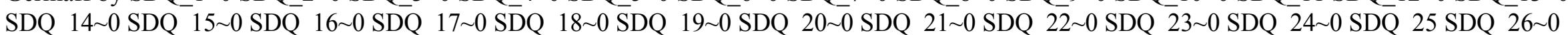
SDQ 27 0 SDQ 28 0 SDQ 29 0 SDQ 30 0 SDQ 31 0 SDQ 32 0 SDQ 33 0 SDQ 34 0 SDQ 35 0 SDQ 36 0 SDQ 37 0 SDQ 38 0 SDQ 39 0

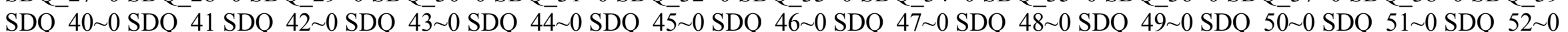
SDQ_53 0 SDQ_54 0 SDQ 55 $~ 0$ SDQ_56 0 SDQ 57 SDQ_58 0 SDQ_59 0 SDQ_60 0 SDQ_61 0 SDQ_62 0 SDQ_63 0 SDQ_64 0 SDQ_65 SDQ $66 \sim 0$ SDQ $67 \sim 0$ SDQ $68 \sim 0$ SDQ $69 \sim 0$ SDQ $70 \sim 0$ SDQ $71 \sim 0$ SDQ $72 \sim 0$ SDQ $73 \sim 0$ SDQ $74 \sim 0$ SDQ $75 \sim 0$ SDQ $76 \sim 0$ (*t1);

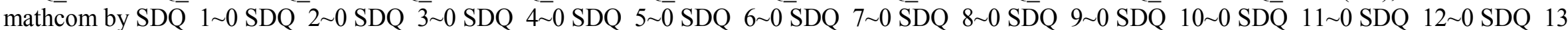

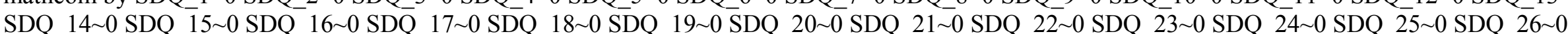
SDQ 27 SDQ 28 0 SDQ 29 0 SDQ 30 0 SDQ 31 0 SDQ 32 0 SDQ 33 0 SDQ 34 0 SDQ 35 0 SDQ 36 0 SDQ 37 0 SDQ 38 0 SDQ 39 0 SDQ 40 0 SDQ 41 0 SDQ 42 0 SDQ 43 SDQ 44 0 SDQ 45 0 SDQ 46 0 SDQ 47 0 SDQ 48 0 SDQ 49 0 SDQ 50 0 SDQ 51 0 SDQ 52 0 
SDQ_53 0 SDQ 54 $\sim$ SDQ_55 0 SDQ 56 $\sim 0$ SDQ_57 0 SDQ 58 $\sim 0$ SDQ_59 SDQ_60 0 SDQ_61 0 SDQ_62 0 SDQ_63 0 SDQ_64 0 SDQ_65 0 SDQ 66 0 SDQ 67 0 SDQ 68 0 SDQ 69 0 SDQ 70 0 SDQ 71 0 SDQ 72 0 SDQ 73 0 SDQ 74 0 SDQ 75 SDQ 76 0 $(* 11)$;

mathaff by SDQ_1 0 SDQ 2 $\sim 0$ SDQ_3 0 SDQ 4 4 0 SDQ_5 0 SDQ 6 SDQ 7 70 SDQ $8 \sim 0$ SDQ 9 $\sim 0$ SDQ_10 0 SDQ_11 0 SDQ_12 0 SDQ_13 0 SDQ 14 0 SDQ 15 0 SDQ 16 0 SDQ 17 0 SDQ 18 0 SDQ 19 0 SDQ 20 SDQ 21 0 SDQ 22 0 SDQ 23 0 SDQ 24 0 SDQ 25 0 SDQ 26 0 SDQ_27 0 SDQ_28 0 SDQ_29 0 SDQ_30 0 SDQ_31 0 SDQ_32 0 SDQ_33 0 SDQ_34 0 SDQ_35 SDQ_36 0 SDQ_37 0 SDQ_38 0 SDQ_39 0 SDQ 40 0 SDQ 41 0 SDQ 42 0 SDQ 43 0 SDQ 44 0 SDQ 45 0 SDQ 46 0 SDQ 47 0 SDQ 48 0 SDQ 49 0 SDQ 50 0 SDQ 51 SDQ 52 0 SDQ_53 0 SDQ_54 0 SDQ_55 0 SDQ_56 0 SDQ 57 0 SDQ_58 0 SDQ_59 0 SDQ_60 0 SDQ_61 0 SDQ_62 0 SDQ_63 0 SDQ_64 0 SDQ_65 0 SDQ 66 0 SDQ 67 0 SDQ 68 SDQ _69 0 SDQ_70 0 SDQ 71 0 SDQ 72 0 SDQ 73 0 SDQ 74 0 SDQ_75 0 SDQ 76 0 $(* t 1)$;

! Method Factor (negative items)

MF BY SDQ 30* SDQ 17 SDQ 12 SDQ 21 SDQ 47 SDQ 23 SDQ 33 SDQ 65

SDQ_75 SDQ_6 SDQ_37 SDQ_61; MF@1; [MF@0];

MFWITH GLOBAL@0 esteem@0 peer@0 appear@0 phy@0 parent@0 schocom@0

schoaff@0.Germcom@0 Germaff@0 MathAff@0 MathCom@0;

! correlated uniquenesses between parallel worded items

SDQ_11 with SDQ_51 SDQ_71; SDQ_51 with SDQ _71;

SDQ 25 with SDQ 35 SDQ 39; SDQ 35 with SDQ 39;

SDQ_41 with SDQ_68 SDQ_9; SDQ_68 with SDQ_9;

SDQ_57 with SDQ_20 SDQ_55; SDQ_20 with SDQ 55;

SDQ 23 with SDQ 6 SDQ 65; SDQ 6 with SDQ 65;

SDQ_4 with SDQ_27 SDQ_16; SDQ_27 with SDQ_16;

SDQ 18 with SDQ 59 SDQ 2; SDQ 59 with SDQ 2;

SDQ_49 with SDQ_13 SDQ_63; SDQ_13 with SDQ_63;

SDQ 73 with SDQ 43 SDQ 31; SDQ 43 with SDQ 31;

SDQ_47 with SDQ_75 SDQ_33; SDQ_75 with SDQ_33; 
Title: Multiple Group Configural Invariance Model of the SDQ-I (Real Data)

data: file $=$ SDQ1.dat;

variable: names = Gender SDQ_1 SDQ_2 SDQ 3 SDQ 4 SDQ 5 SDQ 6

SDQ 7 SDQ 8 SDQ 9 SDQ 10 SDQ 11 SDQ 12 SDQ 13 SDQ 14 SDQ 15 SDQ 16

SDQ_17 SDQ_18 SDQ_19 SDQ_20 SDQ_21 SDQ_22 SDQ_23 SDQ_24 SDQ_25 SDQ_26

SDQ $27 \mathrm{SDQ} 28 \mathrm{SDQ} 29 \mathrm{SDQ} 30 \mathrm{SDQ} 31 \mathrm{SDQ} 32 \mathrm{SDQ} 33 \mathrm{SDQ} 34 \mathrm{SDQ} 35 \mathrm{SDQ} 36$

SDQ_37 SDQ_38 SDQ_39 SDQ_40 SDQ_41 SDQ_42 SDQ_43 SDQ_44 SDQ_45 SDQ_46

SDQ $47 \mathrm{SDQ} 48 \mathrm{SDQ} 49 \mathrm{SDQ} 50 \mathrm{SDQ} 51 \mathrm{SDQ} 52 \mathrm{SDQ} 53 \mathrm{SDQ} 54 \mathrm{SDQ} 55 \mathrm{SDQ} 56$

SDQ_57 SDQ_58 SDQ_59 SDQ_60 SDQ_61 SDQ_62 SDQ_63 SDQ_64 SDQ_65 SDQ_66

SDQ 67 SDQ_68 SDQ 69 SDQ 70 SDQ_71 SDQ 72 SDQ 73 SDQ 74 SDQ 75 SDQ 76;

missing = all (99);

usevar $=\mathrm{SDQ} 1 \mathrm{SDQ} 2 \mathrm{SDQ} 3 \mathrm{SDQ} 4 \mathrm{SDQ} 5 \mathrm{SDQ} 6$

SDQ 7 SDQ 8 SDQ 9 SDQ_10 SDQ_11 SDQ_12 SDQ_13 SDQ_14 SDQ_15 SDQ_16

SDQ_17 SDQ 18 SDQ_19 SDQ_20 SDQ 21 SDQ 22 SDQ 23 SDQ 24 SDQ 25 SDQ 26

SDQ_27 SDQ_28 SDQ_29 SDQ_30 SDQ_31 SDQ_32 SDQ_33 SDQ_34 SDQ_35 SDQ_36

SDQ_37 SDQ_38 SDQ_39 SDQ_40 SDQ 41 SDQ 42 SDQ 43 SDQ 44 SDQ 45 SDQ 46

$\mathrm{SDQ} 47 \mathrm{SDQ} 48 \mathrm{SDQ} 49 \mathrm{SDQ} 50 \mathrm{SDQ} 51 \mathrm{SDQ} 52 \mathrm{SDQ} 53 \mathrm{SDQ} 54 \mathrm{SDQ} 55 \mathrm{SDQ} 56$

SDQ_57 SDQ_58 SDQ_59 SDQ_60 SDQ_61 SDQ_62 SDQ_63 SDQ_64 SDQ_65 SDQ_66

SDQ_67 SDQ_68 SDQ_69 SDQ_70 SDQ_71 SDQ_72 SDQ_73 SDQ_74 SDQ_75 SDQ_76;

grouping $=$ Gender $(1=$ male $2=$ female $)$;

Analysis: ESTIMATOR = MLR; ROTATION = TARGET (orthogonal);

Model:

GLOBAL BY SDQ 1 SDQ 2 SDQ 3 SDQ 4 SDQ 5 SDQ 6 SDQ 7 SDQ 8 SDQ 9 SDQ 10

SDQ_11 SDQ_12 SDQ_13 SDQ_14 SDQ_15 SDQ_16 SDQ_17 SDQ_18 SDQ_19 SDQ_20

SDQ $21 \mathrm{SDQ} 22 \mathrm{SDQ} 23 \mathrm{SDQ} 24 \mathrm{SDQ} 25 \mathrm{SDQ} 26 \mathrm{SDQ} 27 \mathrm{SDQ} 28 \mathrm{SDQ} 29 \mathrm{SDQ} 30$

SDQ_31 SDQ_32 SDQ_33 SDQ_34 SDQ_35 SDQ_36 SDQ_37 SDQ_38 SDQ_39 SDQ_40

SDQ $41 \mathrm{SDQ} 42 \mathrm{SDQ} 43 \mathrm{SDQ} 44 \mathrm{SDQ} 45 \mathrm{SDQ} 46 \mathrm{SDQ} 47 \mathrm{SDQ} 48 \mathrm{SDQ} 49 \mathrm{SDQ} 50$

SDQ_51 SDQ 52 SDQ 53 SDQ 54 SDQ 55 SDQ 56 SDQ 57 SDQ 58 SDQ_59 SDQ_60

SDQ 61 SDQ 62 SDQ 63 SDQ 64 SDQ 65 SDQ 66 SDQ 67 SDQ 68 SDQ 69 SDQ 70

SDQ_71 SDQ 72 SDQ_73 SDQ_74 SDQ_75 SDQ 76 $(* 11)$;

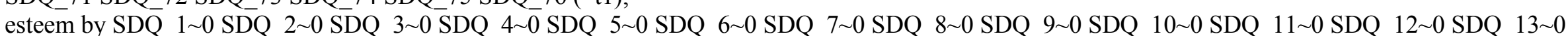
SDQ 14 0 SDQ_15 0 SDQ_16 0 SDQ 17 0 SDQ 18 0 SDQ_19 0 SDQ 20 0 SDQ 21 0 SDQ 22 0 SDQ 23 0 SDQ 24 0 SDQ 25 0 SDQ 26 0 SDQ 27 0 SDQ 28 0 SDQ 29 SDQ 30 0 SDQ 31 0 SDQ 32 0 SDQ 33 0 SDQ 34 0 SDQ 35 0 SDQ 36 0 SDQ 37 SDQ 38 0 SDQ 39 0 SDQ 40 0 SDQ 41 0 SDQ 42 0 SDQ 43 0 SDQ 44 0 SDQ 45 SDQ 46 0 SDQ 47 0 SDQ 48 0 SDQ 49 0 SDQ 50 0 SDQ 51 0 SDQ 52 0 SDQ_53 SDQ_54 0 SDQ 55 $\sim 0 \mathrm{SDQ}$ 56 $\sim 0 \mathrm{SDQ} 5770 \mathrm{SDQ} 58 \sim 0 \mathrm{SDQ} 59 \sim 0 \mathrm{SDQ} \_60 \sim 0 \mathrm{SDQ} \_61 \mathrm{SDQ} \_62 \sim 0 \mathrm{SDQ} 63 \sim 0 \mathrm{SDQ} 64 \sim 0 \mathrm{SDQ} 65 \sim 0$ 
SDQ 66 0 SDQ 67 SDQ 68 0 SDQ_69 0 SDQ 70 SDQ 71 0 SDQ 72 SDQ 73 0 SDQ 74 SDQ 75 0 SDQ 76 (*t1);

peer by SDQ_1 0 SDQ 2 $\sim 0 \mathrm{SDQ}$ 3 0 SDQ 4 0 SDQ 5 0 SDQ 6 0 SDQ_7 SDQ_8 0 SDQ_9 0 SDQ_10 0 SDQ_11 0 SDQ_12 0 SDQ_13 0 SDQ_14 SDQ_15 0 SDQ_16 0 SDQ_17 0 SDQ_18 0 SDQ_19 0 SDQ_20 0 SDQ_21 SDQ_22 0 SDQ_23 0 SDQ_24 0 SDQ_25 0 SDQ_26 0 SDQ 27 0 SDQ 28 SDQ 29 0 SDQ 30 0 SDQ 31 0 SDQ 32 0 SDQ 33 0 SDQ_34 0 SDQ 35 0 SDQ 36 SDQ 37 0 SDQ_38 0 SDQ 39 0 SDQ_40 0 SDQ_41 0 SDQ_42 0 SDQ_43 0 SDQ_44 SDQ_45 0 SDQ_46 0 SDQ_47 0 SDQ_48 0 SDQ_49 0 SDQ_50 0 SDQ_51 0 SDQ_52 SDQ 53 0 SDQ 54 0 SDQ 55 0 SDQ 56 0 SDQ 57 0 SDQ 58 0 SDQ 59 0 SDQ 60 SDQ 61 0 SDQ 62 0 SDQ 63 0 SDQ 64 0 SDQ 65 0 SDQ_66 0 SDQ_67 0 SDQ_68 0 SDQ_69 SDQ_70 0 SDQ 71 0 SDQ_72 0 SDQ_73 0 SDQ_74 0 SDQ_75 0 SDQ_76 0 $(* 1)$;

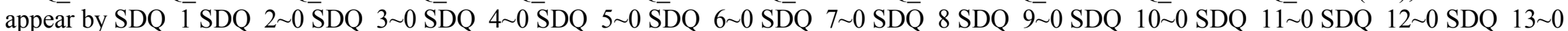
SDQ_14 0 SDQ 15 SDQ 16 0 SDQ_17 0 SDQ_18 0 SDQ_19 0 SDQ_20 0 SDQ_21 0 SDQ_22 SDQ_23 0 SDQ_24 0 SDQ_25 0 SDQ_26 0 SDQ 27 $\sim 0$ SDQ 28 0 SDQ 29 0 SDQ 30 SDQ 31 0 SDQ 32 0 SDQ 33 0 SDQ 34 0 SDQ 35 0 SDQ 36 0 SDQ_37 0 SDQ 38 SDQ 39 0 SDQ_40 0 SDQ_41 0 SDQ_42 0 SDQ 43 0 SDQ 44 0 SDQ 45 0 SDQ 46 SDQ 47 0 SDQ_48 0 SDQ_49 0 SDQ_50 0 SDQ_51 0 SDQ 52 0 SDQ 53 0 SDQ 54 SDQ 55 0 SDQ 56 0 SDQ 57 0 SDQ 58 0 SDQ 59 0 SDQ 60 0 SDQ 61 0 SDQ 62 SDQ 63 0 SDQ 64 0 SDQ 65 0 SDQ_66 0 SDQ_67 0 SDQ_68 0 SDQ 69 0 SDQ_70 0 SDQ_71 0 SDQ_72 0 SDQ 73 0 SDQ 74 $~ 0$ SDQ_75 0 SDQ_76 0 (*t1);

phy by SDQ_1 0 SDQ_2 0 SDQ_3 SDQ_4 0 SDQ_5 0 SDQ_6 0 SDQ_7 0 SDQ_8 0 SDQ_9 0 SDQ_10 SDQ_11 0 SDQ_12 0 SDQ_13 0 SDQ 14 0 SDQ $15 \sim 0$ SDQ 16 0 SDQ_17 SDQ_18 0 SDQ_19 0 SDQ_20 0 SDQ_21 0 SDQ $22 \sim 0$ SDQ_23 0 SDQ 24 SDQ_25 0 SDQ 26 0 SDQ 27 0 SDQ_28 0 SDQ_29 0 SDQ 30 0 SDQ 31 0 SDQ 32 SDQ 33 0 SDQ_34 0 SDQ 35 0 SDQ_36 0 SDQ_37 0 SDQ_38 0 SDQ 39 0 SDQ 40 SDQ 4 41 0 SDQ 42 0 SDQ 43 0 SDQ 44 0 SDQ 45 0 SDQ 46 0 SDQ 47 0 SDQ 48 SDQ 49 0 SDQ 50 0 SDQ 51 0 SDQ 52 0 SDQ 53 0 SDQ 54 $\sim 0$ SDQ 55 0 SDQ 56 SDQ 57 $\sim 0$ SDQ 58 0 SDQ 59 0 SDQ 60 0 SDQ 61 $\sim 0$ SDQ_62 0 SDQ 63 0 SDQ 64 SDQ 65 0 SDQ_66 0 SDQ_67 0 SDQ 68 0 SDQ_69 0 SDQ 70 0 SDQ 71 0 SDQ 72 0 SDQ_73 0 SDQ 74 0 SDQ_75 0 SDQ 76 0 (*t1);

parent by SDQ_1 0 SDQ_2 0 SDQ_3 0 SDQ_4 0 SDQ 5 SDQ 6 0 SDQ 7 7 0 SDQ 8 0 SDQ 9 9 0 SDQ_10 0 SDQ_11 0 SDQ_12 SDQ_13 0 SDQ_14 0 SDQ_15 0 SDQ_16 0 SDQ_17 0 SDQ 18 0 SDQ_19 SDQ 20 0 SDQ 21 0 SDQ_22 0 SDQ 23 0 SDQ_24 0 SDQ_25 0 SDQ_26 SDQ_27 0 SDQ_28 0 SDQ_29 0 SDQ_30 0 SDQ_31 0 SDQ_32 0 SDQ_33 0 SDQ_34 SDQ_35 0 SDQ_36 0 SDQ_37 0 SDQ_38 0 SDQ_39 0 SDQ $40 \sim 0$ SDQ $41 \sim 0$ SDQ 42 SDQ 43 0 SDQ 44 0 SDQ 45 0 SDQ 46 0 SDQ 47 $\sim 0$ SDQ 48 0 SDQ $49 \sim 0$ SDQ 50 SDQ $51 \sim 0$ SDQ $52 \sim 0$ SDQ_53 0 SDQ 54 0 SDQ_55 0 SDQ 56 $\sim 0$ SDQ_57 0 SDQ_58 SDQ_59 0 SDQ_60 0 SDQ_61 0 SDQ_62 0 SDQ_63 0 SDQ_64 0 SDQ_65 0 SDQ 66 SDQ $67 \sim 0$ SDQ $68 \sim 0$ SDQ $69 \sim 0$ SDQ 70 0 SDQ 71 0 SDQ $72 \sim 0$ SDQ $73 \sim 0$ SDQ $74 \sim 0$ SDQ $75 \sim 0$ SDQ $76 \sim 0(* 11)$;

schocom by SDQ_1 0 SDQ_2 SDQ_3 0 SDQ_4 0 SDQ_5 0 SDQ_6 0 SDQ_7 0 SDQ_8 0 SDQ_9 0 SDQ_10 0 SDQ_11 0 SDQ_12 0 SDQ_13 0 SDQ 14 0 SDQ $15 \sim 0$ SDQ 16 SDQ 17 0 SDQ 18 0 SDQ 19 0 SDQ 20 0 SDQ 21 0 SDQ 22 0 SDQ 23 0 SDQ 24 0 SDQ 25 0 SDQ 26 0 SDQ_27 0 SDQ_28 0 SDQ_29 0 SDQ_30 0 SDQ_31 SDQ_32 0 SDQ_33 0 SDQ_34 0 SDQ_35 0 SDQ_36 0 SDQ_37 0 SDQ_38 0 SDQ_39 0

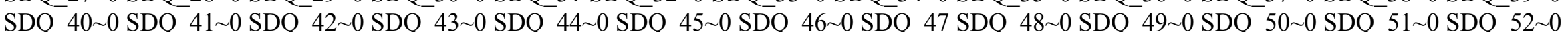
SDQ_53 0 SDQ_54 0 SDQ_55 0 SDQ_56 0 SDQ_57 0 SDQ_58 0 SDQ_59 0 SDQ_60 0 SDQ_61 0 SDQ_62 0 SDQ_63 SDQ_64 0 SDQ_65 0 SDQ 66 0 SDQ 67 0 SDQ 68 0 SDQ 69 0 SDQ 70 0 SDQ 71 0 SDQ 72 0 SDQ 73 0 SDQ 74 0 SDQ $75 \sim 0$ SDQ $76 \sim 0(* t 1)$;

schoaff by SDQ_1 0 SDQ_2 0 SDQ 3 0 SDQ 4 0 SDQ_5 0 SDQ 6 0 SDQ_7 0 SDQ 8 0 SDQ_9 SDQ_10 0 SDQ_11 0 SDQ_12 0 SDQ_13 0 SDQ 14 0 SDQ 15 0 SDQ 16 0 SDQ 17 0 SDQ 18 0 SDQ 19 0 SDQ 20 0 SDQ 21 0 SDQ 22 0 SDQ 23 SDQ 24 0 SDQ 25 0 SDQ 26 0 SDQ 27 0 SDQ 28 0 SDQ 29 0 SDQ 30 0 SDQ 31 0 SDQ 32 0 SDQ 33 0 SDQ 34 0 SDQ 35 0 SDQ 36 0 SDQ 37 0 SDQ 38 0 SDQ 39 SDQ_40 0 SDQ_41 0 SDQ_42 0 SDQ_43 0 SDQ_44 0 SDQ_45 0 SDQ_46 0 SDQ_47 0 SDQ_48 0 SDQ_49 0 SDQ_50 0 SDQ_51 0 SDQ_52 0 
SDQ_53 0 SDQ 54 $\sim$ SDQ_55 SDQ 56 0 SDQ_57 0 SDQ 58 0 SDQ_59 0 SDQ_60 0 SDQ_61 0 SDQ_62 0 SDQ_63 0 SDQ_64 0 SDQ_65 0 SDQ 66 0 SDQ 67 0 SDQ 68 0 SDQ 69 0 SDQ 70 0 SDQ 71 SDQ 72 0 SDQ 73 0 SDQ 74 0 SDQ 75 0 SDQ 76 0 (*t1);

Germcom by SDQ_1 0 SDQ_2 0 SDQ_3 0 SDQ 4 SDQ_5 0 SDQ_6 0 SDQ_7 0 SDQ_8 0 SDQ_9 0 SDQ_10 0 SDQ_11 0 SDQ_12 0 SDQ_13 0 SDQ 14 0 SDQ 15 0 SDQ 16 0 SDQ 17 0 SDQ 18 SDQ 19 0 SDQ 20 0 SDQ 21 0 SDQ 22 0 SDQ 23 0 SDQ 24 0 SDQ 25 0 SDQ 26 0 SDQ_27 0 SDQ_28 0 SDQ_29 0 SDQ_30 0 SDQ_31 0 SDQ_32 0 SDQ_33 SDQ_34 0 SDQ_35 0 SDQ_36 0 SDQ_37 0 SDQ_38 0 SDQ_39 0

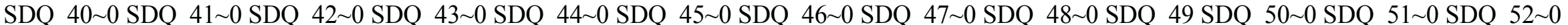
SDQ_53 0 SDQ_54 0 SDQ_55 0 SDQ_56 0 SDQ_57 0 SDQ_58 0 SDQ_59 0 SDQ_60 0 SDQ_61 0 SDQ_62 0 SDQ_63 0 SDQ_64 0 SDQ_65 0 SDQ 66 0 SDQ 67 0 SDQ 68 0 SDQ 69 0 SDQ 70 0 SDQ 71 0 SDQ 72 0 SDQ 73 SDQ 74 0 SDQ 75 0 SDQ 76 0 $(* 11)$;

Germaff by SDQ_1 0 SDQ_2 0 SDQ_3 0 SDQ_4 0 SDQ_5 0 SDQ_6 0 SDQ_7 0 SDQ_8 0 SDQ_9 0 SDQ_10 0 SDQ_11 SDQ_12 0 SDQ_13 0 SDQ_14 0 SDQ 15 0 SDQ_16 0 SDQ 17 0 SDQ_18 0 SDQ 19 0 SDQ 20 0 SDQ 21 0 SDQ 22 0 SDQ 23 0 SDQ 24 0 SDQ 25 SDQ 26 0 SDQ_27 0 SDQ_28 0 SDQ 29 0 SDQ 30 0 SDQ 31 0 SDQ 32 0 SDQ 33 0 SDQ 34 0 SDQ 35 0 SDQ 36 0 SDQ_37 0 SDQ 38 0 SDQ_39 0

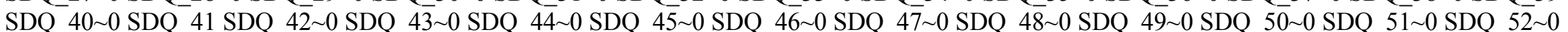
SDQ_53 0 SDQ_54 0 SDQ_55 0 SDQ_56 0 SDQ_57 SDQ_58 0 SDQ 59 0 SDQ_60 0 SDQ_61 0 SDQ_62 0 SDQ_63 0 SDQ_64 0 SDQ_65 SDQ 66 0 SDQ 67 0 SDQ 68 0 SDQ 69 0 SDQ 70 0 SDQ 71 0 SDQ 72 0 SDQ 73 0 SDQ 74 0 SDQ 75 0 SDQ 76 0 (*t1); mathcom by SDQ 1 0 SDQ 2 0 SDQ 3 0 SDQ 4 0 SDQ 5 0 SDQ 6 0 SDQ 7 0 SDQ 8 0 SDQ 9 0 SDQ 10 0 SDQ 11 0 SDQ 12 0 SDQ 13 SDQ_14 0 SDQ 15 0 SDQ_16 0 SDQ 17 0 SDQ 18 0 SDQ 19 0 SDQ 20 0 SDQ 21 0 SDQ 22 0 SDQ 23 0 SDQ 24 0 SDQ 25 0 SDQ 26 0 SDQ 27 SDQ 28 0 SDQ $29 \sim 0$ SDQ 30 0 SDQ 31 0 SDQ 32 $\sim 0$ SDQ 33 0 SDQ 34 $\sim 0$ SDQ 35 0 SDQ 36 0 SDQ 37 0 SDQ 38 0 SDQ 39 0 SDQ_40 0 SDQ 41 $0 \mathrm{SDQ}$ 42 0 SDQ 43 SDQ_44 0 SDQ 45 0 SDQ 46 0 SDQ_47 0 SDQ_48 0 SDQ_49 0 SDQ_50 0 SDQ_51 0 SDQ_52 0 SDQ 53 0 SDQ 54 0 SDQ 55 0 SDQ 56 0 SDQ 57 0 SDQ 58 0 SDQ 59 SDQ 60 0 SDQ 61 0 SDQ 62 0 SDQ 63 0 SDQ 64 0 SDQ 65 0 SDQ_66 0 SDQ_67 0 SDQ_68 0 SDQ_69 0 SDQ_70 0 SDQ_71 0 SDQ 72 0 SDQ_73 0 SDQ_74 0 SDQ_75 SDQ_76 0 $(* 11)$; mathaff by SDQ 1 0 SDQ 2 0 SDQ 3 0 SDQ 4 0 SDQ 5 0 SDQ 6 SDQ $7 \sim 0$ SDQ 8 0 SDQ 9 0 SDQ 10 0 SDQ 11 0 SDQ 12 0 SDQ 13 0 SDQ_14 0 SDQ 15 0 SDQ_16 0 SDQ_17 0 SDQ_18 0 SDQ 19 0 SDQ 20 SDQ 21 0 SDQ 22 0 SDQ_23 0 SDQ_24 0 SDQ 25 0 SDQ_26 0 SDQ 27 0 SDQ 28 0 SDQ 29 0 SDQ 30 0 SDQ 31 0 SDQ 32 0 SDQ 33 0 SDQ 34 0 SDQ 35 SDQ 36 0 SDQ 37 0 SDQ 38 0 SDQ 39 0 SDQ_40 0 SDQ_41 0 SDQ_42 0 SDQ_43 0 SDQ_44 0 SDQ_45 0 SDQ_46 0 SDQ_47 0 SDQ_48 0 SDQ_49 0 SDQ_50 0 SDQ_51 SDQ_52 0 SDQ 53 0 SDQ 54 0 SDQ 55 0 SDQ 56 0 SDQ 57 0 SDQ 58 0 SDQ 59 0 SDQ 60 0 SDQ 61 0 SDQ 62 0 SDQ 63 0 SDQ $64 \sim 0$ SDQ 65 0 SDQ_66 0 SDQ_67 0 SDQ_68 SDQ_69 0 SDQ_70 0 SDQ_71 0 SDQ_72 0 SDQ_73 0 SDQ_74 0 SDQ_75 0 SDQ_76 0 (*t1); ! Method Factor (negative items)

MF BY SDQ 30* SDQ 17 SDQ_12 SDQ 21 SDQ 47 SDQ_23 SDQ 33 SDQ 65 SDQ_75 SDQ_6 SDQ_37 SDQ_61; MF@1; [MF@0];

MFWITH GLOBAL@0 esteem@0 peer@0 appear@0 phy@0 parent@0 schocom@0 schoaff@0 Germcom@0 Germaff@0 MathAff@0 MathCom@0;

! correlated uniquenesses between parallel worded items SDQ 11 with SDQ 51 SDQ 71; SDQ 51 with SDQ 71; SDQ 25 with SDQ 35 SDQ 39; SDQ 35 with SDQ 39; SDQ_41 with SDQ_68 SDQ_9; SDQ_68 with SDQ_9; 
SDQ_57 with SDQ_20 SDQ_55; SDQ_20 with SDQ_55; SDQ 23 with SDQ 6 SDQ 65 ; SDQ 6 with SDQ 65 ;

SDQ_4 with SDQ_27 SDQ_16; SDQ_27 with SDQ_16; SDQ_18 with SDQ_59 SDQ_2; SDQ 59 with SDQ 2;

SDQ_49 with SDQ_13 SDQ_63; SDQ_13 with SDQ_63; SDQ 73 with SDQ 43 SDQ 31; SDQ 43 with SDQ 31; SDQ_47 with SDQ_75 SDQ_33; SDQ_75 with SDQ_33; ! free intercepts

[SDQ_1-SDQ_76*]; [global-mathaff@0];

model female:

GLOBAL BY SDQ 1 SDQ 2 SDQ 3 SDQ 4 SDQ 5 SDQ 6 SDQ 7 SDQ 8 SDQ 9 SDQ 10

SDQ_11 SDQ_12 SDQ_13 SDQ_14 SDQ_15 SDQ_16 SDQ_17 SDQ_18 SDQ_19 SDQ_20

SDQ_21 SDQ_22 SDQ_23 SDQ_24 SDQ_25 SDQ_26 SDQ_27 SDQ_28 SDQ_29 SDQ_30

SDQ_31 SDQ_32 SDQ_33 SDQ_34 SDQ_35 SDQ_36 SDQ_37 SDQ_38 SDQ_39 SDQ_40

$\mathrm{SDQ} 41 \mathrm{SDQ} 42 \mathrm{SDQ} 43 \mathrm{SDQ} 44 \mathrm{SDQ} 45 \mathrm{SDQ} 46 \mathrm{SDQ} 47 \mathrm{SDQ} 48 \mathrm{SDQ} 49 \mathrm{SDQ} 50$

SDQ 51 SDQ 52 SDQ 53 SDQ_54 SDQ 55 SDQ_56 SDQ 57 SDQ 58 SDQ 59 SDQ_60

SDQ_61 SDQ_62 SDQ_63 SDQ_64 SDQ_65 SDQ_66 SDQ_67 SDQ 68 SDQ_69 SDQ 70

SDQ_71 SDQ 72 SDQ 73 SDQ 74 SDQ 75 SDQ 76 (*t1);

esteem by SDQ_1 0 SDQ_2 0 SDQ_3 0 SDQ 4 4 0 SDQ_5 0 SDQ_6 0 SDQ 7 7 0 SDQ_8 0 SDQ 9 0 SDQ_10 0 SDQ_11 0 SDQ_12 0 SDQ_13 0 SDQ 14 0 SDQ 15 0 SDQ 16 0 SDQ 17 0 SDQ 18 0 SDQ 19 0 SDQ 20 0 SDQ 21 0 SDQ 22 0 SDQ 23 0 SDQ 24 0 SDQ 25 0 SDQ 26 0 SDQ_27 0 SDQ_28 0 SDQ_29 SDQ_30 0 SDQ_31 $\sim 0$ SDQ_32 0 SDQ_33 0 SDQ_34 0 SDQ_35 0 SDQ_36 0 SDQ_37 SDQ_38 0 SDQ_39 0 SDQ $40 \sim 0$ SDQ $41 \sim 0$ SDQ $42 \sim 0$ SDQ $43 \sim 0$ SDQ $44 \sim 0$ SDQ 45 SDQ $46 \sim 0$ SDQ $47 \sim 0$ SDQ $48 \sim 0$ SDQ $49 \sim 0$ SDQ $50 \sim 0$ SDQ $51 \sim 0$ SDQ $52 \sim 0$ SDQ 53 SDQ 54 $\sim 0$ SDQ 55 $\sim 0$ SDQ 56 $\sim 0$ SDQ 57 $\sim 0$ SDQ 58 $\sim 0$ SDQ_59 0 SDQ_60 0 SDQ_61 SDQ 62 0 SDQ_63 $\sim 0$ SDQ_64 0 SDQ_65 0 SDQ 66 0 SDQ 67 SDQ 68 0 SDQ 69 0 SDQ 70 SDQ 71 0 SDQ 72 SDQ 73 0 SDQ 74 SDQ 75 0 SDQ $76(* t 1)$;

peer by SDQ_1 0 SDQ_2 0 SDQ_3 0 SDQ_4 0 SDQ_5 0 SDQ_6 0 SDQ_7 SDQ_8 0 SDQ_9 0 SDQ_10 0 SDQ_11 0 SDQ_12 0 SDQ_13 0 SDQ $14 \mathrm{SDQ} \quad 15 \sim 0 \mathrm{SDQ} \quad 16 \sim 0 \mathrm{SDQ} 17 \sim 0 \mathrm{SDQ} 18 \sim 0 \mathrm{SDQ} 19 \sim 0 \mathrm{SDQ} 20 \sim 0 \mathrm{SDQ} 21 \mathrm{SDQ} 22 \sim 0 \mathrm{SDQ} 23 \sim 0 \mathrm{SDQ} 24 \sim 0 \mathrm{SDQ} 25 \sim 0 \mathrm{SDQ} 26 \sim 0$ SDQ_27 0 SDQ_28 SDQ 29 0 SDQ 30 0 SDQ 31 0 SDQ_32 0 SDQ 33 0 SDQ_34 0 SDQ_35 0 SDQ 36 SDQ_37 0 SDQ_38 0 SDQ 39 0 SDQ $40 \sim 0$ SDQ $41 \sim 0$ SDQ $42 \sim 0$ SDQ $43 \sim 0$ SDQ 44 SDQ $45 \sim 0$ SDQ $46 \sim 0$ SDQ $47 \sim 0$ SDQ $48 \sim 0$ SDQ $49 \sim 0$ SDQ $50 \sim 0$ SDQ $51 \sim 0$ SDQ 52 SDQ_53 0 SDQ_54 0 SDQ_55 0 SDQ_56 0 SDQ_57 0 SDQ 58 0 SDQ_59 0 SDQ_60 SDQ_61 0 SDQ_62 0 SDQ_63 0 SDQ_64 0 SDQ_65 0 SDQ $66 \sim 0$ SDQ $67 \sim 0$ SDQ $68 \sim 0$ SDQ 69 SDQ 70 0 SDQ 71 0 SDQ 72 0 SDQ 73 0 SDQ 74 0 SDQ $75 \sim 0$ SDQ $76 \sim 0(* t 1)$;

appear by SDQ 1 SDQ 2 0 SDQ 3 0 SDQ 4 0 SDQ 5 0 SDQ 6 0 SDQ 7 7 0 SDQ 8 SDQ 9 9 SDQ 10 0 SDQ_11 0 SDQ_12 0 SDQ_13 0 SDQ 14 0 SDQ 15 SDQ 16 0 SDQ 17 0 SDQ 18 0 SDQ 19 0 SDQ 20 0 SDQ 21 0 SDQ 22 SDQ 23 0 SDQ 24 0 SDQ 25 0 SDQ 26 0 SDQ 27 0 SDQ 28 0 SDQ 29 0 SDQ 30 SDQ 31 0 SDQ 32 0 SDQ 33 0 SDQ 34 0 SDQ 35 0 SDQ 36 0 SDQ 37 0 SDQ 38 SDQ 39 0 SDQ_40 0 SDQ_41 0 SDQ_42 0 SDQ_43 0 SDQ_44 0 SDQ_45 0 SDQ_46 SDQ_47 0 SDQ_48 0 SDQ_49 0 SDQ_50 0 SDQ_51 0 SDQ_52 0 
SDQ _53 0 SDQ 54 SDQ 55 0 SDQ 56 0 SDQ 57 0 SDQ 58 0 SDQ 59 0 SDQ 60 0 SDQ_61 0 SDQ 62 SDQ_63 0 SDQ_64 0 SDQ_65 0 SDQ 66 0 SDQ 67 0 SDQ 68 0 SDQ 69 0 SDQ 70 0 SDQ 71 0 SDQ 72 0 SDQ 73 0 SDQ 74 0 SDQ 75 0 SDQ 76 0 (*t1);

phy by SDQ_1 0 SDQ_2 0 SDQ 3 SDQ_4 0 SDQ_5 0 SDQ_6 6 SDQ_7 0 SDQ_8 0 SDQ_9 0 SDQ_10 SDQ_11 0 SDQ_12 0 SDQ_13 0 SDQ_14 0 SDQ 15 0 SDQ_16 0 SDQ 17 SDQ 18 0 SDQ 19 0 SDQ_20 0 SDQ 21 0 SDQ 22 0 SDQ_23 0 SDQ 24 SDQ_25 0 SDQ 26 0 SDQ 27 0 SDQ_28 0 SDQ_29 0 SDQ_30 0 SDQ_31 0 SDQ_32 SDQ_33 0 SDQ_34 0 SDQ_35 0 SDQ_36 0 SDQ_37 0 SDQ_38 0 SDQ_39 0 SDQ_40 SDQ 41 0 SDQ 42 0 SDQ 43 0 SDQ 44 0 SDQ 45 0 SDQ 46 0 SDQ 47 0 SDQ 48 SDQ 49 0 SDQ 50 0 SDQ 51 0 SDQ 52 0 SDQ 53 0 SDQ 54 $\sim 0$ SDQ 55 0 SDQ 56 SDQ 57 $\sim 0$ SDQ_58 0 SDQ_59 0 SDQ_60 0 SDQ 61 $\sim 0$ SDQ_62 0 SDQ_63 0 SDQ_64 SDQ_65 0 SDQ_66 0 SDQ $67 \sim 0$ SDQ $68 \sim 0$ SDQ $69 \sim 0$ SDQ 70 0 SDQ 71 0 SDQ 72 0 SDQ 73 0 SDQ 74 0 SDQ 75 0 SDQ 76 0 $(* t 1)$;

parent by SDQ_1 0 SDQ_2 0 SDQ_3 0 SDQ_4 0 SDQ_5 SDQ_6 0 SDQ 7 0 SDQ_8 0 SDQ_9 0 SDQ_10 0 SDQ_11 0 SDQ_12 SDQ_13 0 SDQ 14 0 SDQ_15 0 SDQ 16 0 SDQ 17 0 SDQ_18 0 SDQ_19 SDQ 20 0 SDQ 21 $\sim 0$ SDQ_22 0 SDQ 23 0 SDQ 24 0 SDQ 25 0 SDQ 26 SDQ_27 0 SDQ_28 0 SDQ_29 0 SDQ 30 0 SDQ_31 0 SDQ_32 0 SDQ_33 0 SDQ_34 SDQ_35 0 SDQ_36 0 SDQ_37 0 SDQ_38 0 SDQ_39 0 SDQ 40 0 SDQ 41 0 SDQ 42 SDQ 43 0 SDQ 44 0 SDQ 45 0 SDQ 46 0 SDQ 47 $\sim 0$ SDQ 48 0 SDQ 49 0 SDQ 50 SDQ $51 \sim 0$ SDQ $52 \sim 0$ SDQ_53 0 SDQ_54 0 SDQ_55 0 SDQ_56 0 SDQ_57 0 SDQ 58 SDQ_59 0 SDQ_60 0 SDQ_61 0 SDQ_62 0 SDQ_63 0 SDQ_64 0 SDQ_65 0 SDQ 66 SDQ $67 \sim 0$ SDQ $68 \sim 0$ SDQ 69 0 SDQ 70 0 SDQ 71 0 SDQ 72 0 SDQ 73 0 SDQ 74 0 SDQ 75 0 SDQ 76 0 (*t1);

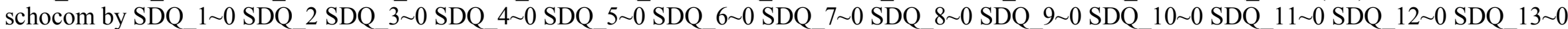
SDQ_14 0 SDQ_15 0 SDQ_16 SDQ_17 0 SDQ_18 0 SDQ_19 0 SDQ_20 0 SDQ_21 0 SDQ_22 0 SDQ_23 0 SDQ_24 0 SDQ_25 0 SDQ_26 0 SDQ 27 0 SDQ 28 0 SDQ 29 0 SDQ 30 0 SDQ 31 SDQ 32 0 SDQ 33 0 SDQ 34 0 SDQ 35 0 SDQ 36 0 SDQ 37 0 SDQ 38 0 SDQ 39 0 SDQ_40 0 SDQ_41 0 SDQ_42 0 SDQ_43 0 SDQ_44 0 SDQ_45 0 SDQ 46 0 SDQ 47 SDQ_48 0 SDQ_49 0 SDQ_50 0 SDQ_51 0 SDQ_52 0 SDQ 53 0 SDQ 54 0 SDQ 55 0 SDQ 56 0 SDQ 57 0 SDQ 58 0 SDQ_59 0 SDQ 60 0 SDQ_61 0 SDQ_62 0 SDQ_63 SDQ_64 0 SDQ 65 0 SDQ_66 0 SDQ_67 0 SDQ_68 0 SDQ_69 0 SDQ_70 0 SDQ_71 0 SDQ_72 0 SDQ_73 0 SDQ_74 0 SDQ_75 0 SDQ_76 0 (*t1);

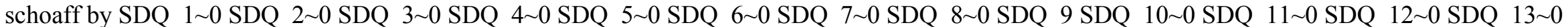
SDQ 14 0 SDQ 15 0 SDQ_16 0 SDQ_17 0 SDQ_18 0 SDQ 19 0 SDQ 20 0 SDQ 21 $\sim 0$ SDQ 22 0 SDQ 23 SDQ 24 0 SDQ 25 0 SDQ 26 0 SDQ 27 0 SDQ 28 0 SDQ 29 0 SDQ 30 0 SDQ 31 0 SDQ 32 0 SDQ 33 0 SDQ 34 0 SDQ 35 0 SDQ 36 0 SDQ 37 0 SDQ 38 0 SDQ 39 SDQ_40 0 SDQ_41 0 SDQ_42 0 SDQ_43 0 SDQ 44 0 SDQ_45 0 SDQ_46 0 SDQ_47 0 SDQ_48 0 SDQ_49 0 SDQ_50 0 SDQ_51 0 SDQ_52 0 SDQ $53 \sim 0$ SDQ $54 \sim 0$ SDQ 55 SDQ 56 0 SDQ 57 0 SDQ 58 0 SDQ 59 0 SDQ 60 0 SDQ 61 0 SDQ $62 \sim 0$ SDQ $63 \sim 0$ SDQ $64 \sim 0$ SDQ $65 \sim 0$ SDQ_66 0 SDQ_67 0 SDQ_68 0 SDQ_69 0 SDQ 70 0 SDQ_71 SDQ_72 0 SDQ_73 0 SDQ_74 0 SDQ_75 0 SDQ_76 0 (*t1);

Germcom by SDQ 1 0 SDQ 2 0 SDQ 3 0 SDQ 4 SDQ 5 0 SDQ 6 0 SDQ 7 0 SDQ 8 0 SDQ_9 0 SDQ 10 0 SDQ_11 0 SDQ 12 0 SDQ_13 0 SDQ_14 0 SDQ_15 0 SDQ_16 0 SDQ_17 0 SDQ_18 SDQ_19 0 SDQ_20 0 SDQ_21 0 SDQ_22 0 SDQ_23 0 SDQ_24 0 SDQ 25 0 SDQ 26 0 SDQ 27 0 SDQ 28 0 SDQ 29 0 SDQ 30 0 SDQ 31 0 SDQ 32 0 SDQ 33 SDQ 34 0 SDQ 35 0 SDQ 36 0 SDQ 37 0 SDQ 38 0 SDQ 39 0 SDQ_40 0 SDQ_41 0 SDQ_42 0 SDQ 43 0 SDQ_44 0 SDQ_45 0 SDQ_46 0 SDQ 47 0 SDQ_48 0 SDQ 49 SDQ_50 0 SDQ_51 0 SDQ_52 0 SDQ 53 0 SDQ 54 0 SDQ_55 0 SDQ 56 0 SDQ 57 0 SDQ_58 0 SDQ 59 0 SDQ 60 0 SDQ_61 0 SDQ 62 0 SDQ 63 0 SDQ_64 0 SDQ_65 0 SDQ_66 0 SDQ_67 0 SDQ 68 0 SDQ_69 0 SDQ 70 0 SDQ_71 0 SDQ 72 0 SDQ 73 SDQ_74 0 SDQ_75 0 SDQ_76 0 (*t1);

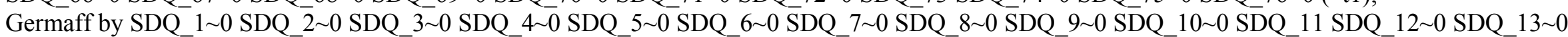
SDQ 14 0 SDQ 15 0 SDQ 16 0 SDQ 17 0 SDQ 18 0 SDQ 19 0 SDQ 20 0 SDQ 21 0 SDQ 22 0 SDQ 23 0 SDQ 24 0 SDQ 25 SDQ 26 0 SDQ_27 0 SDQ_28 0 SDQ_29 0 SDQ_30 0 SDQ_31 0 SDQ_32 0 SDQ_33 0 SDQ_34 0 SDQ_35 0 SDQ_36 0 SDQ_37 0 SDQ_38 0 SDQ_39 0 
SDQ_40 0 SDQ $41 \mathrm{SDQ}$ 42 0 SDQ 43 0 SDQ 44 0 SDQ 45 0 SDQ 46 0 SDQ 47 0 SDQ_48 0 SDQ 49 0 SDQ_50 0 SDQ_51 0 SDQ_52 0 SDQ_53 0 SDQ_54 0 SDQ_55 0 SDQ_56 0 SDQ 57 SDQ_58 0 SDQ_59 0 SDQ_60 0 SDQ_61 0 SDQ_62 0 SDQ_63 0 SDQ_64 0 SDQ_65 SDQ_66 0 SDQ 67 0 SDQ_68 0 SDQ 69 0 SDQ_70 0 SDQ_71 0 SDQ_72 0 SDQ_73 0 SDQ_74 0 SDQ_75 0 SDQ_76 0 (*t1);

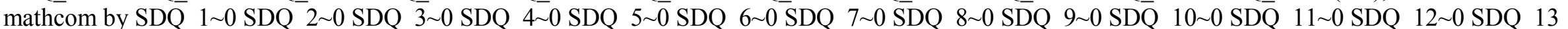
SDQ_14 0 SDQ_15 0 SDQ_16 0 SDQ_17 0 SDQ_18 0 SDQ_19 0 SDQ_20 0 SDQ_21 0 SDQ_22 0 SDQ_23 0 SDQ_24 0 SDQ_25 0 SDQ_26 0 SDQ 27 SDQ 28 0 SDQ 29 0 SDQ 30 0 SDQ 31 0 SDQ 32 0 SDQ 33 0 SDQ 34 0 SDQ 35 0 SDQ 36 0 SDQ 37 0 SDQ 38 0 SDQ 39 0 SDQ_40 0 SDQ_41 0 SDQ 42 0 SDQ 43 SDQ_44 0 SDQ_45 0 SDQ_46 0 SDQ_47 0 SDQ_48 0 SDQ_49 0 SDQ_50 0 SDQ_51 0 SDQ_52 0 SDQ 53 0 SDQ 54 0 SDQ 55 0 SDQ 56 0 SDQ 57 0 SDQ 58 0 SDQ 59 SDQ 60 0 SDQ 61 0 SDQ 62 0 SDQ 63 0 SDQ 64 0 SDQ 65 0 SDQ_66 0 SDQ_67 0 SDQ_68 0 SDQ_69 0 SDQ_70 0 SDQ_71 0 SDQ_72 0 SDQ_73 0 SDQ_74 0 SDQ_75 SDQ_76 0 (*t1);

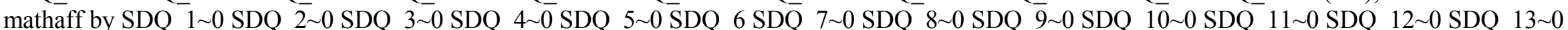
SDQ_14 0 SDQ_15 0 SDQ_16 0 SDQ_17 0 SDQ_18 0 SDQ_19 0 SDQ_20 SDQ 21 $\sim 0$ SDQ_22 0 SDQ_23 0 SDQ_24 0 SDQ_25 0 SDQ_26 0 SDQ_27 0 SDQ_28 0 SDQ_29 0 SDQ_30 0 SDQ_31 0 SDQ_32 0 SDQ_33 0 SDQ_34 0 SDQ_35 SDQ_36 0 SDQ_37 0 SDQ_38 0 SDQ_39 0 SDQ_40 0 SDQ_41 0 SDQ_42 0 SDQ_43 0 SDQ_44 0 SDQ_45 0 SDQ_46 0 SDQ_47 0 SDQ_48 0 SDQ_49 0 SDQ_50 0 SDQ_51 SDQ_52 0 SDQ_53 0 SDQ_54 0 SDQ_55 0 SDQ_56 0 SDQ 57 0 SDQ_58 0 SDQ_59 0 SDQ_60 0 SDQ_61 0 SDQ_62 0 SDQ_63 0 SDQ_64 0 SDQ_65 0 SDQ_66 0 SDQ_67 0 SDQ_68 SDQ_69 0 SDQ_70 0 SDQ_71 0 SDQ_72 0 SDQ_73 0 SDQ_74 0 SDQ_75 0 SDQ_76 0 (*t1);

! Method Factor (negative items)

MF BY SDQ 30* SDQ 17 SDQ 12 SDQ 21 SDQ 47 SDQ 23 SDQ 33 SDQ 65

SDQ_75 SDQ_6 SDQ_37 SDQ_61; MF@1; [MF@0];

MFWITH GLOBAL@0 esteem@0 peer@0 appear@0 phy@0 parent@0 schocom@0 schoaff@0.Germcom@0 Germaff@0 MathAff@0 MathCom@0;

! correlated uniquenesses between parallel worded items

SDQ_11 with SDQ_51 SDQ_71; SDQ_51 with SDQ_71;

SDQ 25 with SDQ 35 SDQ 39 ; SDQ 35 with SDQ 39;

SDQ_41 with SDQ_68 SDQ_9; SDQ_68 with SDQ_9;

SDQ 57 with SDQ 20 SDQ 55; SDQ 20 with SDQ 55;

SDQ_23 with SDQ_6 SDQ_65; SDQ_6 with SDQ_65;

SDQ 4 with SDQ 27 SDQ 16; SDQ 27 with SDQ 16;

SDQ_18 with SDQ_59 SDQ_2; SDQ_59 with SDQ_2;

SDQ_49 with SDQ_13 SDQ_63; SDQ_13 with SDQ_63;

SDQ_73 with SDQ_43 SDQ_31; SDQ_43 with SDQ_31;

SDQ_47 with SDQ_75 SDQ_33; SDQ_75 with SDQ_33;

! free intercepts

[SDQ 1-SDQ 76*];

[global-mathaff@0]; 
Title: Multiple Group Weak (Loadings) Invariance Model of the SDQ-I (Real Data) ! [...]Model section only

GLOBAL BY SDQ_1 SDQ_2 SDQ 3 SDQ 4 SDQ_5 SDQ_6 SDQ 7 SDQ_8 SDQ_9 SDQ_10

SDQ_11 SDQ_12 SDQ_13 SDQ_14 SDQ_15 SDQ_16 SDQ_17 SDQ_18 SDQ_19 SDQ_20

SDQ_21 SDQ_22 SDQ_23 SDQ_24 SDQ_25 SDQ_26 SDQ_27 SDQ_28 SDQ_29 SDQ_30

SDQ $31 \mathrm{SDQ} 32 \mathrm{SDQ} 33 \mathrm{SDQ} 34 \mathrm{SDQ} 35 \mathrm{SDQ} 36 \mathrm{SDQ} 37 \mathrm{SDQ} 38 \mathrm{SDQ} 39 \mathrm{SDQ} 40$

SDQ_41 SDQ_42 SDQ_43 SDQ_44 SDQ_45 SDQ_46 SDQ_47 SDQ_48 SDQ_49 SDQ_50

SDQ 51 SDQ 52 SDQ 53 SDQ 54 SDQ 55 SDQ 56 SDQ 57 SDQ 58 SDQ 59 SDQ 60

SDQ_61 SDQ_62 SDQ_63 SDQ_64 SDQ_65 SDQ_66 SDQ_67 SDQ_68 SDQ_69 SDQ_70

SDQ 71 SDQ 72 SDQ 73 SDQ 74 SDQ 75 SDQ $76(* t 1)$;

esteem by SDQ $1 \sim 0$ SDQ 2 2 0 SDQ 3 0 SDQ 4 0 SDQ 5 0 SDQ_6 0 SDQ 7 7 0 SDQ_8 0 SDQ_9 0 SDQ_10 0 SDQ_11 0 SDQ_12 0 SDQ_13 0 SDQ 14 0 SDQ 15 0 SDQ 16 0 SDQ 17 0 SDQ 18 0 SDQ 19 0 SDQ 20 0 SDQ 21 0 SDQ 22 0 SDQ 23 0 SDQ 24 0 SDQ 25 0 SDQ 26 0 SDQ_27 0 SDQ_28 0 SDQ_29 SDQ_30 0 SDQ_31 $\sim 0$ SDQ_32 0 SDQ_33 0 SDQ_34 0 SDQ_35 0 SDQ_36 0 SDQ_37 SDQ_38 0 SDQ_39 0 SDQ $40 \sim 0$ SDQ $41 \sim 0$ SDQ $42 \sim 0$ SDQ $43 \sim 0$ SDQ 44 0 SDQ 45 SDQ $46 \sim 0$ SDQ $47 \sim 0$ SDQ $48 \sim 0$ SDQ $49 \sim 0$ SDQ $50 \sim 0$ SDQ $51 \sim 0$ SDQ $52 \sim 0$ SDQ_53 SDQ 54 0 SDQ 55 0 SDQ 56 0 SDQ 57 0 SDQ 58 0 SDQ_59 0 SDQ_60 0 SDQ_61 SDQ_62 0 SDQ_63 0 SDQ_64 0 SDQ_65 0 SDQ 66 0 SDQ 67 SDQ $68 \sim 0$ SDQ 69 0 SDQ 70 SDQ $71 \sim 0$ SDQ 72 SDQ 73 0 SDQ 74 SDQ 75 0 SDQ $76(* 1)$;

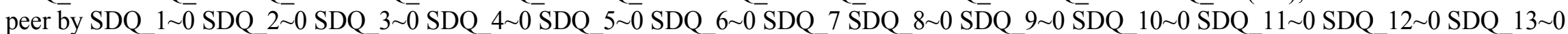
SDQ_14 SDQ_15 0 SDQ_16 0 SDQ_17 0 SDQ_18 0 SDQ_19 0 SDQ 20 0 SDQ_21 SDQ_22 0 SDQ_23 0 SDQ_24 0 SDQ_25 0 SDQ_26 0 SDQ 27 0 SDQ 28 SDQ 29 0 SDQ 30 0 SDQ 31 0 SDQ 32 0 SDQ 33 0 SDQ 34 0 SDQ 35 0 SDQ 36 SDQ 37 0 SDQ 38 0 SDQ 39 0 SDQ 40 0 SDQ 41 0 SDQ 42 0 SDQ 43 0 SDQ 44 SDQ 45 0 SDQ 46 0 SDQ 47 0 SDQ_48 0 SDQ_49 0 SDQ_50 0 SDQ_51 0 SDQ_52 SDQ 53 0 SDQ 54 0 SDQ 55 0 SDQ 56 0 SDQ 57 0 SDQ 58 0 SDQ 59 0 SDQ 60 SDQ 61 0 SDQ 62 0 SDQ 63 0 SDQ 64 0 SDQ 65 0 SDQ_66 0 SDQ_67 0 SDQ_68 0 SDQ_69 SDQ_70 0 SDQ_71 0 SDQ_72 0 SDQ 73 0 SDQ_74 0 SDQ_75 0 SDQ_76 0 (*t1);

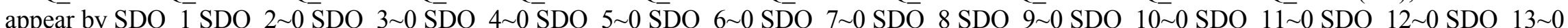
SDQ_14 0 SDQ 15 SDQ $16 \sim 0$ SDQ_17 0 SDQ_18 0 SDQ_19 0 SDQ 20 0 SDQ_21 0 SDQ 22 SDQ_23 0 SDQ 24 0 SDQ 25 0 SDQ_26 0 SDQ 27 0 SDQ 28 0 SDQ 29 0 SDQ 30 SDQ 31 0 SDQ 32 0 SDQ 33 0 SDQ 34 0 SDQ 35 0 SDQ 36 0 SDQ 37 0 SDQ 38 SDQ 39 0 SDQ $40 \sim 0 \mathrm{SDQ}$ 41 0 SDQ_42 0 SDQ_43 0 SDQ 44 0 SDQ 45 0 SDQ 46 SDQ 47 0 SDQ_48 0 SDQ_49 0 SDQ_50 0 SDQ_51 0 SDQ 52 0 SDQ 53 0 SDQ 54 SDQ 55 0 SDQ 56 0 SDQ 57 0 SDQ 58 0 SDQ 59 0 SDQ 60 0 SDQ 61 0 SDQ 62 SDQ 63 0 SDQ $64 \sim 0$ SDQ $65 \sim 0$ SDQ_66 0 SDQ_67 0 SDQ_68 0 SDQ_69 0 SDQ_70 0 SDQ_71 0 SDQ_72 0 SDQ 73 0 SDQ_74 0 SDQ 75 0 SDQ_76 0 (*t1);

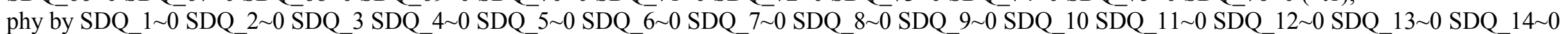
SDQ_15 0 SDQ_16 0 SDQ_17 SDQ_18 0 SDQ_19 0 SDQ_20 0 SDQ_21 0 SDQ 22 0 SDQ_23 0 SDQ_24 SDQ_25 0 SDQ 26 0 SDQ_27 0 SDQ 28 0 SDQ 29 0 SDQ 30 0 SDQ 31 0 SDQ $32 \mathrm{SDQ}$ 33 0 SDQ 34 0 SDQ 35 0 SDQ 36 0 SDQ 37 0 SDQ 38 0 SDQ 39 0 SDQ 40 SDQ_41 0 SDQ_42 0 SDQ_43 0 SDQ 44 0 SDQ_45 0 SDQ 46 0 SDQ_47 0 SDQ 48 SDQ_49 0 SDQ_50 0 SDQ_51 0 SDQ_52 0 SDQ 53 0 SDQ_54 0 SDQ_55 0 SDQ_56 SDQ_57 0 SDQ 58 0 SDQ_59 0 SDQ_60 0 SDQ_61 0 SDQ_62 0 SDQ_63 0 SDQ_64 SDQ_65 0 SDQ_66 0 SDQ_67 0 SDQ 68 0 SDQ_69 0 SDQ_70 0 SDQ 71 0 SDQ 72 0 SDQ_73 0 SDQ_74 0 SDQ_75 0 SDQ_76 0 (*t1); parent by SDQ_1 0 SDQ_2 0 SDQ_3 0 SDQ_4 0 SDQ_5 SDQ_6 0 SDQ_7 0 SDQ_8 0 SDQ_9 0 SDQ_10 0 SDQ_11 0 SDQ_12 SDQ_13 0 
SDQ_14 0 SDQ_15 0 SDQ_16 0 SDQ_17 0 SDQ 18 0 SDQ_19 SDQ 20 0 SDQ 21 0 SDQ_22 0 SDQ 23 0 SDQ_24 0 SDQ 25 0 SDQ_26 SDQ 27 0 SDQ 28 0 SDQ 29 0 SDQ 30 0 SDQ 31 0 SDQ 32 0 SDQ 33 0 SDQ 34 SDQ 35 0 SDQ 36 0 SDQ 37 0 SDQ 38 0 SDQ 39 0 SDQ_40 0 SDQ_41 0 SDQ_42 SDQ_43 0 SDQ 44 $\sim 0$ SDQ_45 0 SDQ_46 0 SDQ 47 $\sim 0$ SDQ_48 0 SDQ_49 0 SDQ_50 SDQ 51 $\sim 0$ SDQ_52 0 SDQ 53 0 SDQ 54 0 SDQ 55 0 SDQ 56 0 SDQ 57 0 SDQ 58 SDQ 59 0 SDQ 60 0 SDQ 61 0 SDQ 62 0 SDQ 63 0 SDQ 64 0 SDQ 65 0 SDQ_66 SDQ_67 0 SDQ_68 0 SDQ_69 0 SDQ_70 0 SDQ 71 0 SDQ_72 0 SDQ_73 0 SDQ_74 0 SDQ_75 0 SDQ 76 0 (*t1);

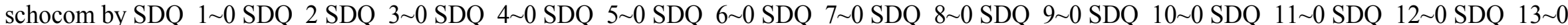
SDQ 14 0 SDQ $15 \sim 0$ SDQ 16 SDQ $17 \sim 0$ SDQ 18 0 SDQ 19 0 SDQ 20 0 SDQ 21 0 SDQ 22 0 SDQ 23 0 SDQ 24 0 SDQ 25 0 SDQ 26 0 SDQ 27 0 SDQ 28 0 SDQ 29 0 SDQ 30 0 SDQ 31 SDQ 32 0 SDQ 33 0 SDQ 34 0 SDQ 35 0 SDQ 36 0 SDQ 37 0 SDQ 38 0 SDQ 39 0 SDQ _40 0 SDQ 41 0 SDQ 42 0 SDQ 43 0 SDQ 44 0 SDQ 45 0 SDQ 46 0 SDQ 47 SDQ 48 0 SDQ 49 0 SDQ 50 0 SDQ 51 0 SDQ 52 0 SDQ 53 0 SDQ 54 0 SDQ 55 0 SDQ 56 0 SDQ 57 0 SDQ 58 0 SDQ 59 0 SDQ $60 \sim 0$ SDQ 61 0 SDQ 62 0 SDQ 63 SDQ 64 0 SDQ 65 0 SDQ_66 0 SDQ_67 0 SDQ_68 0 SDQ 69 0 SDQ 70 0 SDQ 71 0 SDQ 72 0 SDQ 73 0 SDQ 74 0 SDQ 75 0 SDQ 76 0 (*t1); schoaff by SDQ 1 $\sim 0$ SDQ 2 0 SDQ 3 0 SDQ 4 0 SDQ 5 0 SDQ $6 \sim 0$ SDQ $7 \sim 0$ SDQ $8 \sim 0$ SDQ 9 SDQ $10 \sim 0$ SDQ $11 \sim 0$ SDQ $12 \sim 0$ SDQ $13 \sim 0$ SDQ_14 0 SDQ_15 0 SDQ_16 0 SDQ_17 0 SDQ_18 0 SDQ_19 0 SDQ_20 0 SDQ 21 $\sim 0$ SDQ_22 0 SDQ_23 SDQ_24 0 SDQ_25 0 SDQ_26 0 SDQ_27 0 SDQ_28 0 SDQ_29 0 SDQ_30 0 SDQ_31 0 SDQ_32 0 SDQ_33 0 SDQ_34 0 SDQ_35 0 SDQ_36 0 SDQ_37 0 SDQ_38 0 SDQ_39 SDQ 40 0 SDQ 41 0 SDQ 42 0 SDQ 43 0 SDQ 44 0 SDQ 45 0 SDQ 46 0 SDQ 47 0 SDQ 48 0 SDQ 49 0 SDQ 50 0 SDQ 51 0 SDQ 52 0 SDQ_53 0 SDQ_54 0 SDQ_55 SDQ_56 0 SDQ_57 0 SDQ_58 0 SDQ_59 0 SDQ_60 $\sim 0$ SDQ_61 0 SDQ_62 0 SDQ_63 0 SDQ_64 0 SDQ_65 0 SDQ 66 0 SDQ 67 0 SDQ_68 0 SDQ 69 0 SDQ 70 0 SDQ 71 SDQ 72 0 SDQ 73 0 SDQ 74 0 SDQ 75 0 SDQ 76 0 (*t1);

Germcom by SDQ $11 \sim 0 \mathrm{SDQ} \_2 \sim 0 \mathrm{SDQ} 3 \sim 0 \mathrm{SDQ} 4 \mathrm{SDQ} 5 \sim 0 \mathrm{SDQ}$ 6 $\sim 0$ SDQ 7 7 SDQ 8 0 SDQ 9 9 0 SDQ 10 0 SDQ_11 0 SDQ 12 0 SDQ 13 0 SDQ 14 0 SDQ 15 0 SDQ 16 0 SDQ 17 0 SDQ 18 SDQ 19 0 SDQ 20 0 SDQ 21 0 SDQ 22 0 SDQ 23 0 SDQ 24 0 SDQ 25 0 SDQ 26 0 SDQ_27 0 SDQ_28 0 SDQ_29 0 SDQ_30 0 SDQ_31 0 SDQ_32 0 SDQ_33 SDQ_34 0 SDQ_35 0 SDQ_36 0 SDQ_37 0 SDQ_38 0 SDQ 39 0 SDQ 40 0 SDQ 41 0 SDQ 42 0 SDQ 43 0 SDQ 44 0 SDQ 45 0 SDQ 46 0 SDQ 47 0 SDQ 48 0 SDQ 49 SDQ 50 0 SDQ 51 0 SDQ 52 0 SDQ_53 0 SDQ_54 0 SDQ_55 0 SDQ_56 0 SDQ_57 0 SDQ_58 0 SDQ_59 0 SDQ_60 0 SDQ_61 0 SDQ_62 0 SDQ_63 0 SDQ_64 0 SDQ_65 0 SDQ $66 \sim 0$ SDQ 67 0 SDQ 68 0 SDQ 69 0 SDQ 70 0 SDQ 71 0 SDQ $72 \sim 0$ SDQ 73 SDQ 74 0 SDQ 75 0 SDQ 76 0 $(* 11)$; Germaff by SDQ_1 0 SDQ 2 $\sim 0$ SDQ 3 3 0 SDQ_4 0 SDQ 5 0 SDQ_6 0 SDQ 7 0 SDQ_8 0 SDQ_9 0 SDQ_10 0 SDQ_11 SDQ 12 0 SDQ_13 0 SDQ 14 0 SDQ 15 0 SDQ 16 0 SDQ 17 0 SDQ 18 0 SDQ 19 0 SDQ 20 0 SDQ 21 0 SDQ 22 0 SDQ 23 0 SDQ 24 0 SDQ 25 SDQ 26 0 SDQ_27 0 SDQ_28 0 SDQ_29 0 SDQ 30 0 SDQ_31 0 SDQ_32 0 SDQ_33 0 SDQ_34 0 SDQ_35 0 SDQ_36 0 SDQ_37 0 SDQ_38 0 SDQ_39 0

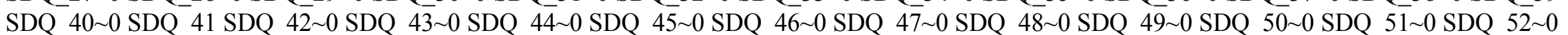
SDQ_53 0 SDQ_54 0 SDQ 55 $\sim 0$ SDQ_56 0 SDQ 57 SDQ_58 0 SDQ_59 0 SDQ_60 0 SDQ_61 0 SDQ_62 0 SDQ_63 0 SDQ_64 0 SDQ_65 SDQ 66 0 SDQ 67 0 SDQ 68 0 SDQ 69 0 SDQ 70 0 SDQ 71 0 SDQ 72 0 SDQ 73 0 SDQ 74 0 SDQ $75 \sim 0$ SDQ $76 \sim 0$ (*t1); mathcom by SDQ_1 0 SDQ_2 0 SDQ_3 0 SDQ 4 0 SDQ_5 0 SDQ_6 0 SDQ_7 0 SDQ 8 0 SDQ_9 0 SDQ_10 0 SDQ_11 0 SDQ_12 0 SDQ_13 SDQ 14 0 SDQ 15 0 SDQ 16 0 SDQ 17 0 SDQ 18 0 SDQ 19 0 SDQ 20 0 SDQ 21 0 SDQ 22 0 SDQ 23 0 SDQ 24 0 SDQ 25 0 SDQ 26 0 SDQ 27 SDQ 28 0 SDQ 29 $\sim 0$ SDQ_30 0 SDQ_31 0 SDQ 32 0 SDQ_33 0 SDQ_34 0 SDQ_35 0 SDQ_36 0 SDQ_37 0 SDQ_38 0 SDQ_39 0 SDQ 40 0 SDQ 41 0 SDQ 42 0 SDQ 43 SDQ 44 0 SDQ 45 0 SDQ 46 0 SDQ 47 0 SDQ 48 0 SDQ 49 0 SDQ 50 0 SDQ 51 0 SDQ 52 0 SDQ 53 0 SDQ 54 0 SDQ 55 0 SDQ 56 0 SDQ 57 0 SDQ 58 0 SDQ 59 SDQ 60 0 SDQ 61 0 SDQ 62 0 SDQ 63 0 SDQ 64 0 SDQ 65 0 SDQ_66 0 SDQ_67 0 SDQ_68 0 SDQ_69 0 SDQ_70 0 SDQ_71 0 SDQ_72 0 SDQ_73 0 SDQ_74 0 SDQ_75 SDQ_76 0 (*t1); 
mathaff by SDQ_1 0 SDQ 2 0 SDQ_3 0 SDQ 4 0 SDQ_5 0 SDQ 6 SDQ_7 0 SDQ 8 0 SDQ_9 0 SDQ_10 0 SDQ_11 0 SDQ_12 0 SDQ_13 0 SDQ 14 0 SDQ 15 0 SDQ 16 0 SDQ 17 0 SDQ 18 0 SDQ 19 0 SDQ 20 SDQ 21 0 SDQ 22 0 SDQ 23 0 SDQ 24 0 SDQ 25 0 SDQ 26 0 SDQ_27 0 SDQ_28 0 SDQ_29 0 SDQ 30 0 SDQ_31 0 SDQ_32 0 SDQ 33 0 SDQ 34 0 SDQ_35 SDQ_36 0 SDQ_37 0 SDQ_38 0 SDQ 39 0 SDQ 40 0 SDQ 41 0 SDQ 42 0 SDQ 43 0 SDQ 44 0 SDQ 45 0 SDQ 46 0 SDQ 47 0 SDQ 48 0 SDQ 49 0 SDQ 50 0 SDQ 51 SDQ 52 0 SDQ_53 0 SDQ_54 0 SDQ_55 0 SDQ_56 0 SDQ 57 0 SDQ_58 0 SDQ_59 0 SDQ_60 0 SDQ_61 0 SDQ_62 0 SDQ_63 0 SDQ_64 0 SDQ_65 0 SDQ 66 0 SDQ 67 0 SDQ 68 SDQ 69 0 SDQ_70 0 SDQ 71 0 SDQ_72 0 SDQ 73 0 SDQ 74 0 SDQ 75 0 SDQ 76 0 (*t1);

! Method Factor (negative items)

MF BY SDQ 30* (MF1)

SDQ_17 SDQ_12 SDQ_21 SDQ_47 SDQ_23 SDQ_33 SDQ_65 SDQ_75 SDQ_6 SDQ_37 SDQ_61 (MF2-MF12);

MF@1; [MF@0];

MFWITH GLOBAL@0 esteem@0 peer@0 appear@0 phy@0 parent@0 schocom@0

schoaff@0 Germcom@0 Germaff@0 MathAff@0 MathCom@0;

! correlated uniquenesses between parallel worded items

SDQ_11 with SDQ_51 SDQ_71; SDQ_51 with SDQ_71;

SDQ_25 with SDQ_35 SDQ_39; SDQ_35 with SDQ_39;

SDQ 41 with SDQ 68 SDQ 9; SDQ 68 with SDQ 9;

SDQ 57 with SDQ 20 SDQ_55; SDQ 20 with SDQ 55;

SDQ_23 with SDQ_6 SDQ_65; SDQ_6 with SDQ_65;

SDQ 4 with SDQ 27 SDQ 16; SDQ 27 with SDQ 16;

SDQ_18 with SDQ_59 SDQ_2; SDQ_59 with SDQ_2;

SDQ 49 with SDQ 13 SDQ 63; SDQ 13 with SDQ 63;

SDQ 73 with SDQ 43 SDQ 31; SDQ 43 with SDQ 31;

SDQ 47 with SDQ 75 SDQ 33; SDQ 75 with SDQ 33;

! free intercepts

[SDQ 1-SDQ 76*];

[global-mathaff@0];

model female:

! Method Factor (negative items)

MF BY SDQ 30* (MF1)

SDQ_17 SDQ_12 SDQ_21 SDQ_47 SDQ_23 SDQ_33 SDQ_65 SDQ_75 SDQ_6 SDQ_37 SDQ_61 (MF2-MF12); MF*; [MF@0];

MFWITH GLOBAL@0 esteem@0 peer@0 appear@0 phy@0 parent@0 schocom@0

schoaff@0. Germcom@0 Germaff@0 MathAff@0 MathCom@0;

! correlated uniquenesses between parallel worded items

SDQ_11 with SDQ_51 SDQ_71; SDQ_51 with SDQ_71; 
SDQ_25 with SDQ_35 SDQ_39; SDQ_35 with SDQ_39; SDQ 41 with SDQ 68 SDQ 9; SDQ 68 with SDQ 9; SDQ_57 with SDQ_20 SDQ_55; SDQ_20 with SDQ 55; SDQ 23 with SDQ 6 SDQ 65; SDQ 6 with SDQ 65; SDQ_4 with SDQ_27 SDQ_16; SDQ_27 with SDQ_16; SDQ 18 with SDQ 59 SDQ 2; SDQ 59 with SDQ 2;

SDQ_49 with SDQ_13 SDQ_63; SDQ_13 with SDQ_63; SDQ 73 with SDQ 43 SDQ 31; SDQ 43 with SDQ 31;

SDQ_47 with SDQ_75 SDQ_33; SDQ_75 with SDQ_33;

! free intercepts

[SDQ_1-SDQ_76*]; [global-mathaff@0]; 
Title: Multiple Group Strong (Loadings, Intercepts) Invariance Model of the SDQ-I (Real Data) ! [...]Model section only

GLOBAL BY SDQ_1 SDQ_2 SDQ_3 SDQ 4 SDQ_5 SDQ_6 SDQ_7 SDQ_8 SDQ_9 SDQ_10

SDQ_11 SDQ_12 SDQ_13 SDQ_14 SDQ_15 SDQ_16 SDQ_17 SDQ_18 SDQ_19 SDQ_20

SDQ_21 SDQ_22 SDQ_23 SDQ_24 SDQ_25 SDQ_26 SDQ_27 SDQ_28 SDQ_29 SDQ_30

SDQ $31 \mathrm{SDQ} 32 \mathrm{SDQ} 33 \mathrm{SDQ} 34 \mathrm{SDQ} 35 \mathrm{SDQ} 36 \mathrm{SDQ} 37 \mathrm{SDQ} 38 \mathrm{SDQ} 39 \mathrm{SDQ} 40$

SDQ_41 SDQ_42 SDQ_43 SDQ_44 SDQ_45 SDQ_46 SDQ_47 SDQ_48 SDQ_49 SDQ_50

SDQ 51 SDQ 52 SDQ 53 SDQ 54 SDQ 55 SDQ 56 SDQ 57 SDQ 58 SDQ 59 SDQ 60

SDQ_61 SDQ_62 SDQ_63 SDQ_64 SDQ_65 SDQ_66 SDQ_67 SDQ_68 SDQ_69 SDQ_70

SDQ 71 SDQ 72 SDQ 73 SDQ 74 SDQ 75 SDQ $76(* 11)$;

esteem by SDQ $1 \sim 0$ SDQ 2 2 0 SDQ 3 0 SDQ 4 0 SDQ 5 0 SDQ_6 0 SDQ 7 7 0 SDQ_8 0 SDQ_9 0 SDQ_10 0 SDQ_11 0 SDQ_12 0 SDQ_13 0 SDQ 14 0 SDQ 15 0 SDQ 16 0 SDQ 17 0 SDQ 18 0 SDQ 19 0 SDQ 20 0 SDQ 21 0 SDQ 22 0 SDQ 23 0 SDQ 24 0 SDQ 25 0 SDQ 26 0 SDQ_27 0 SDQ_28 0 SDQ_29 SDQ_30 0 SDQ_31 0 SDQ_32 0 SDQ_33 0 SDQ_34 0 SDQ_35 0 SDQ_36 0 SDQ_37 SDQ_38 0 SDQ_39 0 SDQ $40 \sim 0$ SDQ $41 \sim 0$ SDQ $42 \sim 0$ SDQ $43 \sim 0$ SDQ 44 0 SDQ 45 SDQ $46 \sim 0$ SDQ $47 \sim 0$ SDQ $48 \sim 0$ SDQ $49 \sim 0$ SDQ $50 \sim 0$ SDQ $51 \sim 0$ SDQ $52 \sim 0$ SDQ_53 SDQ 54 0 SDQ 55 0 SDQ_56 0 SDQ 57 0 SDQ 58 0 SDQ_59 0 SDQ_60 0 SDQ_61 SDQ_62 0 SDQ_63 0 SDQ_64 0 SDQ_65 0 SDQ 66 0 SDQ 67 SDQ 68 0 SDQ 69 0 SDQ 70 SDQ 71 0 SDQ 72 SDQ 73 0 SDQ 74 SDQ 75 0 SDQ $76(* 1)$;

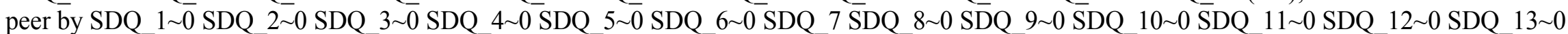
SDQ_14 SDQ_15 0 SDQ_16 0 SDQ_17 0 SDQ_18 0 SDQ_19 0 SDQ_20 0 SDQ_21 SDQ_22 0 SDQ_23 0 SDQ_24 0 SDQ_25 0 SDQ_26 0 SDQ 27 0 SDQ 28 SDQ 29 0 SDQ 30 0 SDQ 31 0 SDQ 32 0 SDQ 33 0 SDQ 34 0 SDQ 35 0 SDQ 36 SDQ 37 0 SDQ 38 0 SDQ 39 0 SDQ_40 0 SDQ_41 0 SDQ_42 0 SDQ_43 0 SDQ_44 SDQ_45 0 SDQ_46 0 SDQ_47 0 SDQ_48 0 SDQ_49 0 SDQ_50 0 SDQ_51 0 SDQ_52 SDQ 53 0 SDQ 54 0 SDQ 55 0 SDQ 56 0 SDQ 57 0 SDQ 58 0 SDQ 59 0 SDQ 60 SDQ 61 0 SDQ 62 0 SDQ 63 0 SDQ 64 0 SDQ 65 0 SDQ 66 6 SDQ 67 0 SDQ 68 0 SDQ 69 SDQ 70 0 SDQ 71 0 SDQ 72 0 SDQ 73 0 SDQ 74 0 SDQ 75 0 SDQ 76 0 $(* 11)$;

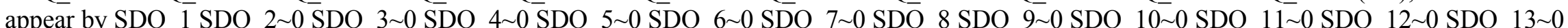
SDQ $14 \sim 0 \mathrm{SDQ} \_15 \mathrm{SDQ}$ 16 0 SDQ 17 0 SDQ 18 0 SDQ_19 0 SDQ 20 0 SDQ 21 0 SDQ 22 SDQ 23 0 SDQ_24 0 SDQ 25 0 SDQ_26 0 SDQ 27 0 SDQ 28 0 SDQ 29 0 SDQ 30 SDQ 31 0 SDQ 32 0 SDQ 33 0 SDQ 34 0 SDQ 35 0 SDQ 36 0 SDQ 37 0 SDQ 38 SDQ 39 0 SDQ $40 \sim 0 \mathrm{SDQ}$ 41 0 SDQ_42 0 SDQ_43 0 SDQ 44 0 SDQ 45 0 SDQ 46 SDQ $47 \sim 0$ SDQ_48 0 SDQ_49 0 SDQ_50 0 SDQ 51 0 SDQ 52 0 SDQ 53 0 SDQ 54 SDQ 55 0 SDQ 56 0 SDQ 57 0 SDQ 58 0 SDQ 59 0 SDQ 60 0 SDQ 61 0 SDQ 62 SDQ 63 0 SDQ $64 \sim 0$ SDQ $65 \sim 0$ SDQ $66 \sim 0$ SDQ 67 0 SDQ 68 0 SDQ 69 0 SDQ 70 0 SDQ 71 $\sim$ SDQ 72 0 SDQ 73 0 SDQ 74 $\sim 0$ SDQ 75 0 SDQ 76 0 (*t1);

phy by SDQ 1 0 SDQ 2 0 SDQ 3 SDQ $4 \sim 0$ SDQ $5 \sim 0$ SDQ $6 \sim 0$ SDQ $7 \sim 0$ SDQ $8 \sim 0$ SDQ 9 0 SDQ 10 SDQ $11 \sim 0$ SDQ $12 \sim 0$ SDQ $13 \sim 0$ SDQ $14 \sim 0$ SDQ_15 0 SDQ_16 0 SDQ_17 SDQ_18 0 SDQ_19 0 SDQ_20 0 SDQ_21 0 SDQ 22 0 SDQ_23 0 SDQ_24 SDQ_25 0 SDQ 26 0 SDQ_27 0 SDQ 28 0 SDQ 29 0 SDQ 30 0 SDQ 31 0 SDQ 32 SDQ 33 0 SDQ 34 0 SDQ 35 0 SDQ 36 0 SDQ 37 0 SDQ 38 0 SDQ 39 0 SDQ 40 SDQ_41 0 SDQ_42 0 SDQ_43 0 SDQ 44 0 SDQ_45 0 SDQ 46 0 SDQ 47 0 SDQ_48 SDQ_49 0 SDQ_50 0 SDQ_51 0 SDQ_52 0 SDQ 53 0 SDQ_54 0 SDQ_55 0 SDQ_56 SDQ_57 0 SDQ 58 $\sim 0$ SDQ_59 0 SDQ 60 0 SDQ_61 0 SDQ_62 0 SDQ_63 0 SDQ_64 SDQ_65 0 SDQ_66 0 SDQ $67 \sim 0$ SDQ 68 0 SDQ 69 0 SDQ 70 0 SDQ 71 0 SDQ 72 0 SDQ 73 0 SDQ 74 0 SDQ 75 0 SDQ 76 0 (*t1); parent by SDQ_1 0 SDQ_2 0 SDQ_3 0 SDQ_4 0 SDQ_5 SDQ_6 0 SDQ_7 0 SDQ_8 0 SDQ_9 0 SDQ_10 0 SDQ_11 0 SDQ_12 SDQ_13 0 
SDQ_14 0 SDQ_15 0 SDQ_16 0 SDQ_17 0 SDQ 18 0 SDQ_19 SDQ 20 0 SDQ 21 0 SDQ_22 0 SDQ 23 0 SDQ_24 0 SDQ_25 0 SDQ_26 SDQ 27 0 SDQ 28 0 SDQ 29 0 SDQ 30 0 SDQ 31 0 SDQ 32 0 SDQ 33 0 SDQ 34 SDQ 35 0 SDQ 36 0 SDQ 37 0 SDQ 38 0 SDQ 39 0 SDQ_40 0 SDQ_41 0 SDQ_42 SDQ_43 0 SDQ 44 $\sim 0$ SDQ 45 0 SDQ 46 0 SDQ 47 $\sim 0$ SDQ_48 0 SDQ_49 0 SDQ_50 SDQ 51 $\sim 0$ SDQ_52 0 SDQ 53 0 SDQ 54 0 SDQ 55 0 SDQ 56 0 SDQ 57 0 SDQ 58 SDQ 59 0 SDQ 60 0 SDQ 61 0 SDQ 62 0 SDQ 63 0 SDQ 64 0 SDQ 65 0 SDQ_66 SDQ_67 0 SDQ_68 0 SDQ_69 0 SDQ_70 0 SDQ 71 0 SDQ_72 0 SDQ_73 0 SDQ_74 0 SDQ_75 0 SDQ_76 0 (*t1);

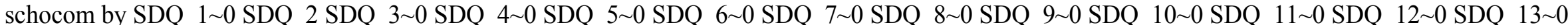
SDQ 14 0 SDQ $15 \sim 0$ SDQ 16 SDQ $17 \sim 0$ SDQ 18 0 SDQ 19 0 SDQ 20 0 SDQ 21 0 SDQ 22 0 SDQ 23 0 SDQ 24 0 SDQ 25 0 SDQ 26 0 SDQ 27 0 SDQ 28 0 SDQ 29 0 SDQ 30 0 SDQ 31 SDQ 32 0 SDQ 33 0 SDQ 34 0 SDQ 35 0 SDQ 36 0 SDQ 37 0 SDQ 38 0 SDQ 39 0 SDQ _40 0 SDQ 41 0 SDQ 42 0 SDQ 43 0 SDQ 44 0 SDQ 45 0 SDQ 46 0 SDQ 47 SDQ 48 0 SDQ 49 0 SDQ 50 0 SDQ 51 0 SDQ 52 0 SDQ 53 0 SDQ 54 0 SDQ 55 0 SDQ 56 0 SDQ 57 0 SDQ 58 0 SDQ 59 0 SDQ 60 0 SDQ 61 0 SDQ 62 0 SDQ 63 SDQ 64 0 SDQ_65 0 SDQ_66 0 SDQ 67 0 SDQ_68 0 SDQ 69 0 SDQ_70 0 SDQ_71 0 SDQ_72 0 SDQ_73 0 SDQ_74 0 SDQ_75 0 SDQ 76 0 (*t1);

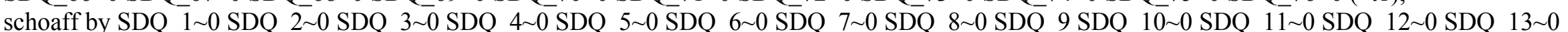
SDQ_14 0 SDQ_15 0 SDQ_16 0 SDQ_17 0 SDQ_18 0 SDQ_19 0 SDQ_20 0 SDQ 21 $\sim 0$ SDQ_22 0 SDQ_23 SDQ_24 0 SDQ_25 0 SDQ_26 0 SDQ_27 0 SDQ_28 0 SDQ_29 0 SDQ_30 0 SDQ_31 0 SDQ_32 0 SDQ_33 0 SDQ_34 0 SDQ_35 0 SDQ_36 0 SDQ_37 0 SDQ_38 0 SDQ_39 SDQ 40 0 SDQ 41 0 SDQ 42 0 SDQ 43 0 SDQ 44 0 SDQ 45 0 SDQ 46 0 SDQ 47 0 SDQ 48 0 SDQ 49 0 SDQ 50 0 SDQ 51 0 SDQ 52 0 SDQ_53 0 SDQ_54 0 SDQ_55 SDQ_56 0 SDQ_57 0 SDQ_58 0 SDQ_59 0 SDQ_60 0 SDQ_61 0 SDQ_62 0 SDQ_63 0 SDQ_64 0 SDQ_65 0 SDQ 66 0 SDQ 67 0 SDQ_68 0 SDQ 69 0 SDQ 70 0 SDQ 71 SDQ 72 0 SDQ 73 0 SDQ 74 0 SDQ 75 0 SDQ 76 0 (*t1);

Germcom by SDQ_1 0 SDQ_2 0 SDQ_3 0 SDQ_4 SDQ 5 0 SDQ_6 0 SDQ_7 0 SDQ_8 0 SDQ_9 0 SDQ_10 0 SDQ_11 0 SDQ 12 0 SDQ_13 0 SDQ 14 0 SDQ 15 0 SDQ_16 0 SDQ_17 0 SDQ 18 SDQ 19 0 SDQ 20 0 SDQ 21 $\sim 0$ SDQ 22 0 SDQ 23 0 SDQ 24 0 SDQ 25 0 SDQ 26 0 SDQ_27 0 SDQ_28 0 SDQ_29 0 SDQ_30 0 SDQ_31 0 SDQ_32 0 SDQ_33 SDQ_34 0 SDQ_35 0 SDQ_36 0 SDQ_37 0 SDQ_38 0 SDQ_39 0 SDQ 40 0 SDQ 41 0 SDQ 42 0 SDQ 43 0 SDQ 44 0 SDQ 45 0 SDQ 46 0 SDQ 47 0 SDQ 48 0 SDQ 49 SDQ 50 0 SDQ 51 0 SDQ 52 0 SDQ_53 0 SDQ_54 0 SDQ_55 0 SDQ_56 0 SDQ_57 0 SDQ_58 0 SDQ_59 0 SDQ_60 0 SDQ_61 0 SDQ_62 0 SDQ_63 0 SDQ_64 0 SDQ_65 0 SDQ 66 0 SDQ 67 0 SDQ 68 0 SDQ 69 0 SDQ 70 0 SDQ 71 0 SDQ 72 0 SDQ 73 SDQ 74 0 SDQ 75 0 SDQ 76 0 $(* 11)$; Germaff by SDQ_1 0 SDQ 2 $\sim 0$ SDQ 3 3 0 SDQ_4 0 SDQ 5 0 SDQ_6 0 SDQ 7 0 SDQ_8 0 SDQ_9 0 SDQ_10 0 SDQ_11 SDQ_12 0 SDQ_13 0 SDQ 14 0 SDQ 15 0 SDQ 16 0 SDQ 17 0 SDQ 18 0 SDQ 19 0 SDQ 20 0 SDQ 21 0 SDQ 22 0 SDQ 23 0 SDQ 24 0 SDQ 25 SDQ 26 0 SDQ_27 0 SDQ_28 0 SDQ_29 0 SDQ_30 0 SDQ_31 0 SDQ_32 0 SDQ_33 0 SDQ_34 0 SDQ_35 0 SDQ_36 0 SDQ_37 0 SDQ_38 0 SDQ_39 0

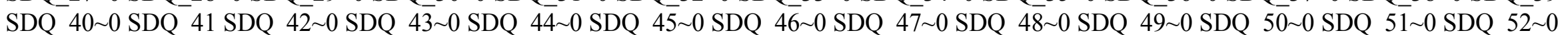
SDQ_53 0 SDQ_54 0 SDQ 55 $\sim 0$ SDQ_56 0 SDQ 57 SDQ_58 0 SDQ_59 0 SDQ_60 0 SDQ_61 0 SDQ_62 0 SDQ_63 0 SDQ_64 0 SDQ_65 SDQ 66 0 SDQ 67 0 SDQ 68 0 SDQ 69 0 SDQ 70 0 SDQ 71 0 SDQ 72 0 SDQ 73 0 SDQ 74 0 SDQ $75 \sim 0$ SDQ $76 \sim 0$ (*t1); mathcom by SDQ_1 0 SDQ 2 2 0 SDQ 3 0 SDQ 4 $\sim 0 \mathrm{SDQ}$ 5 0 SDQ 6 0 SDQ 7 0 SDQ 8 0 SDQ 9 9 SDQ 10 0 SDQ 11 0 SDQ 12 0 SDQ_13 SDQ 14 0 SDQ 15 0 SDQ 16 0 SDQ 17 0 SDQ 18 0 SDQ 19 0 SDQ 20 0 SDQ 21 0 SDQ 22 0 SDQ 23 0 SDQ 24 0 SDQ 25 0 SDQ 26 0 SDQ 27 SDQ 28 $\sim$ SDQ 29 0 SDQ 30 0 SDQ 31 0 SDQ 32 0 SDQ 33 0 SDQ $34 \sim 0$ SDQ 35 0 SDQ 36 0 SDQ 37 $\sim 0$ SDQ 38 0 SDQ 39 0 SDQ 40 0 SDQ 41 0 SDQ 42 0 SDQ 43 SDQ 44 0 SDQ 45 0 SDQ 46 0 SDQ 47 0 SDQ 48 0 SDQ 49 0 SDQ 50 0 SDQ 51 0 SDQ 52 0 SDQ 53 0 SDQ 54 0 SDQ 55 0 SDQ 56 0 SDQ 57 0 SDQ 58 0 SDQ 59 SDQ 60 0 SDQ 61 0 SDQ 62 0 SDQ 63 0 SDQ 64 0 SDQ 65 0 SDQ_66 0 SDQ_67 0 SDQ_68 0 SDQ_69 0 SDQ_70 0 SDQ_71 0 SDQ_72 0 SDQ_73 0 SDQ_74 0 SDQ_75 SDQ_76 0 (*t1); 
mathaff by SDQ_1 0 SDQ 2 0 SDQ_3 0 SDQ 4 0 SDQ_5 0 SDQ 6 SDQ_7 0 SDQ 8 0 SDQ_9 0 SDQ_10 0 SDQ_11 0 SDQ_12 0 SDQ_13 0 SDQ 14 0 SDQ 15 0 SDQ 16 0 SDQ 17 0 SDQ 18 0 SDQ 19 0 SDQ 20 SDQ 21 0 SDQ 22 0 SDQ 23 0 SDQ 24 0 SDQ 25 0 SDQ 26 0 SDQ_27 0 SDQ_28 0 SDQ_29 0 SDQ 30 0 SDQ_31 0 SDQ_32 0 SDQ_33 0 SDQ 34 0 SDQ_35 SDQ_36 0 SDQ_37 0 SDQ_38 0 SDQ_39 0 SDQ 40 0 SDQ 41 0 SDQ 42 0 SDQ 43 0 SDQ 44 0 SDQ 45 0 SDQ 46 0 SDQ 47 0 SDQ 48 0 SDQ 49 0 SDQ 50 0 SDQ 51 SDQ 52 0 SDQ_53 0 SDQ_54 0 SDQ_55 0 SDQ_56 0 SDQ 57 0 SDQ_58 0 SDQ_59 0 SDQ_60 0 SDQ_61 0 SDQ_62 0 SDQ_63 0 SDQ_64 0 SDQ_65 0 SDQ 66 0 SDQ 67 0 SDQ 68 SDQ 69 0 SDQ_70 0 SDQ 71 0 SDQ_72 0 SDQ 73 0 SDQ 74 0 SDQ 75 0 SDQ 76 0 (*t1);

! Method Factor (negative items)

MF BY SDQ 30* (MF1)

SDQ_17 SDQ_12 SDQ_21 SDQ_47 SDQ_23 SDQ_33 SDQ_65 SDQ_75 SDQ_6 SDQ_37 SDQ_61 (MF2-MF12);

MF@1; [MF@0];

MFWITH GLOBAL@0 esteem@0 peer@0 appear@0 phy@0 parent@0 schocom@0

schoaff@0 Germcom@0 Germaff@0 MathAff@0 MathCom@0;

! correlated uniquenesses between parallel worded items

SDQ_11 with SDQ_51 SDQ_71; SDQ_51 with SDQ_71;

SDQ_25 with SDQ_35 SDQ_39; SDQ_35 with SDQ_39;

SDQ 41 with SDQ 68 SDQ 9; SDQ 68 with SDQ 9;

SDQ 57 with SDQ 20 SDQ_55; SDQ 20 with SDQ 55;

SDQ_23 with SDQ_6 SDQ_65; SDQ_6 with SDQ_65;

SDQ 4 with SDQ 27 SDQ 16; SDQ 27 with SDQ 16;

SDQ_18 with SDQ_59 SDQ_2; SDQ_59 with SDQ_2;

SDQ 49 with SDQ 13 SDQ 63; SDQ 13 with SDQ 63;

SDQ_73 with SDQ_43 SDQ_31; SDQ_43 with SDQ_31;

SDQ 47 with SDQ 75 SDQ 33; SDQ 75 with SDQ 33;

model female:

! Method Factor (negative items)

MF BY SDQ $30 *$ (MF1)

SDQ 17 SDQ 12 SDQ 21 SDQ 47 SDQ 23 SDQ 33 SDQ 65 SDQ 75 SDQ 6 SDQ 37 SDQ 61 (MF2-MF12);

$\mathrm{MF}^{*} ;[\mathrm{MF} *]$;

MFWITH GLOBAL@0 esteem@0 peer@0 appear@0 phy@0 parent@0 schocom@0

schoaff@0.Germcom@0 Germaff@0 MathAff@0 MathCom@0;

! correlated uniquenesses between parallel worded items

SDQ_11 with SDQ_51 SDQ_71; SDQ_51 with SDQ_71;

SDQ 25 with SDQ 35 SDQ 39 ; SDQ 35 with SDQ 39;

SDQ 41 with SDQ 68 SDQ 9; SDQ 68 with SDQ 9;

SDQ 57 with SDQ 20 SDQ 55; SDQ 20 with SDQ 55; 
SDQ_23 with SDQ_6 SDQ_65; SDQ_6 with SDQ_65;

SDQ 4 with SDQ 27 SDQ 16; SDQ 27 with SDQ 16;

SDQ_18 with SDQ_59 SDQ_2; SDQ_59 with SDQ 2;

SDQ 49 with SDQ 13 SDQ 63; SDQ 13 with SDQ 63;

SDQ_73 with SDQ_43 SDQ_31; SDQ_43 with SDQ_31;

SDQ 47 with SDQ 75 SDQ 33; SDQ 75 with SDQ 33; 
Title: Multiple Group Strict (Loadings, Intercepts, Uniquenesses) Invariance Model of the SDQ-I (Real Data) ! [...]Model section only

GLOBAL BY SDQ_1 SDQ_2 SDQ_3 SDQ 4 SDQ_5 SDQ_6 SDQ_7 SDQ_8 SDQ_9 SDQ_10

SDQ_11 SDQ_12 SDQ_13 SDQ_14 SDQ_15 SDQ_16 SDQ_17 SDQ_18 SDQ_19 SDQ_20

SDQ_21 SDQ_22 SDQ_23 SDQ_24 SDQ_25 SDQ_26 SDQ_27 SDQ_28 SDQ_29 SDQ_30

SDQ 31 SDQ 32 SDQ 33 SDQ 34 SDQ 35 SDQ 36 SDQ 37 SDQ 38 SDQ 39 SDQ 40

SDQ_41 SDQ_42 SDQ_43 SDQ_44 SDQ_45 SDQ_46 SDQ_47 SDQ_48 SDQ_49 SDQ_50

SDQ 51 SDQ 52 SDQ 53 SDQ 54 SDQ 55 SDQ 56 SDQ 57 SDQ 58 SDQ 59 SDQ 60

SDQ_61 SDQ_62 SDQ_63 SDQ_64 SDQ_65 SDQ_66 SDQ_67 SDQ_68 SDQ_69 SDQ_70

SDQ 71 SDQ 72 SDQ 73 SDQ 74 SDQ 75 SDQ $76(* t 1)$

esteem by SDQ_1 0 SDQ _ 2 0 SDQ 3 0 SDQ 4 0 SDQ_5 0 SDQ 6 0 SDQ 7 0 SDQ 8 0 SDQ 9 0 SDQ_10 0 SDQ_11 0 SDQ_12 0 SDQ_13 0 SDQ 14 0 SDQ 15 0 SDQ 16 0 SDQ 17 0 SDQ 18 0 SDQ 19 0 SDQ 20 0 SDQ 21 0 SDQ 22 0 SDQ 23 0 SDQ 24 0 SDQ 25 0 SDQ 26 0 SDQ_27 0 SDQ_28 0 SDQ_29 SDQ_30 0 SDQ 31 0 SDQ_32 0 SDQ_33 0 SDQ_34 0 SDQ_35 0 SDQ_36 0 SDQ_37 SDQ_38 0 SDQ_39 0 SDQ $40 \sim 0$ SDQ $41 \sim 0$ SDQ $42 \sim 0$ SDQ $43 \sim 0$ SDQ 44 0 SDQ 45 SDQ $46 \sim 0$ SDQ $47 \sim 0$ SDQ $48 \sim 0$ SDQ $49 \sim 0$ SDQ $50 \sim 0$ SDQ $51 \sim 0$ SDQ $52 \sim 0$ SDQ_53 SDQ 54 0 SDQ 55 0 SDQ 56 0 SDQ 57 0 SDQ 58 0 SDQ_59 0 SDQ_60 0 SDQ_61 SDQ_62 0 SDQ_63 0 SDQ_64 0 SDQ_65 0 SDQ 66 0 SDQ 67 SDQ 68 0 SDQ 69 0 SDQ 70 SDQ 71 0 SDQ 72 SDQ 73 0 SDQ 74 SDQ 75 0 SDQ $76(* 1)$;

peer by SDQ 1 0 SDQ 2 2 0 SDQ 3 0 SDQ 4 0 SDQ 5 0 SDQ 6 6 SDQ 7 SDQ 8 0 SDQ 9 0 SDQ 10 0 SDQ 11 0 SDQ 12 0 SDQ 13 0 SDQ_14 SDQ_15 0 SDQ_16 0 SDQ_17 0 SDQ_18 0 SDQ_19 0 SDQ_20 0 SDQ_21 SDQ_22 0 SDQ_23 0 SDQ_24 0 SDQ_25 0 SDQ_26 0 SDQ 27 0 SDQ 28 SDQ 29 0 SDQ 30 0 SDQ 31 0 SDQ 32 0 SDQ 33 0 SDQ 34 0 SDQ 35 0 SDQ 36 SDQ 37 0 SDQ 38 0 SDQ 39 0 SDQ_40 0 SDQ_41 0 SDQ_42 0 SDQ_43 0 SDQ_44 SDQ_45 0 SDQ_46 0 SDQ_47 0 SDQ_48 0 SDQ_49 0 SDQ_50 0 SDQ_51 0 SDQ_52 SDQ 53 0 SDQ 54 0 SDQ 55 0 SDQ 56 0 SDQ 57 0 SDQ 58 0 SDQ 59 0 SDQ 60 SDQ 61 0 SDQ 62 0 SDQ 63 0 SDQ 64 0 SDQ 65 0 SDQ 66 6 SDQ 67 0 SDQ 68 0 SDQ 69 SDQ 70 0 SDQ 71 0 SDQ 72 0 SDQ 73 0 SDQ 74 0 SDQ 75 0 SDQ 76 0 $(* 11)$;

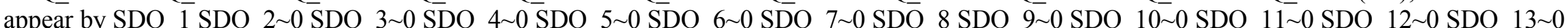
SDQ $14 \sim 0 \mathrm{SDQ} \_15 \mathrm{SDQ}$ 16 0 SDQ 17 0 SDQ 18 0 SDQ_19 0 SDQ 20 0 SDQ 21 0 SDQ 22 SDQ 23 0 SDQ_24 0 SDQ 25 0 SDQ 26 0 SDQ 27 0 SDQ 28 0 SDQ 29 0 SDQ 30 SDQ 31 0 SDQ 32 0 SDQ 33 0 SDQ 34 0 SDQ 35 0 SDQ 36 0 SDQ 37 0 SDQ 38 SDQ 39 0 SDQ $40 \sim 0 \mathrm{SDQ}$ 41 0 SDQ_42 0 SDQ_43 0 SDQ 44 0 SDQ 45 0 SDQ 46 SDQ_47 0 SDQ_48 0 SDQ_49 0 SDQ_50 0 SDQ 51 0 SDQ 52 0 SDQ 53 0 SDQ 54 SDQ 55 0 SDQ 56 0 SDQ 57 0 SDQ 58 0 SDQ 59 0 SDQ 60 0 SDQ 61 0 SDQ 62 SDQ 63 0 SDQ $64 \sim 0$ SDQ $65 \sim 0$ SDQ $66 \sim 0$ SDQ 67 0 SDQ 68 0 SDQ 69 0 SDQ 70 0 SDQ 71 0 SDQ 72 0 SDQ 73 0 SDQ 74 0 SDQ 75 0 SDQ 76 0 (*t1);

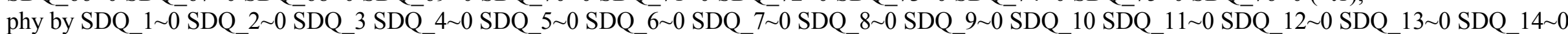
SDQ_15 0 SDQ_16 0 SDQ_17 SDQ_18 0 SDQ_19 0 SDQ_20 0 SDQ_21 0 SDQ 22 0 SDQ_23 0 SDQ_24 SDQ_25 0 SDQ_26 0 SDQ_27 0 SDQ 28 0 SDQ 29 0 SDQ 30 0 SDQ 31 0 SDQ $32 \mathrm{SDQ}$ 33 0 SDQ 34 0 SDQ 35 0 SDQ 36 0 SDQ 37 0 SDQ 38 0 SDQ 39 0 SDQ 40 SDQ_41 0 SDQ_42 0 SDQ_43 0 SDQ 44 0 SDQ_45 0 SDQ 46 0 SDQ 47 0 SDQ_48 SDQ_49 0 SDQ_50 0 SDQ_51 0 SDQ_52 0 SDQ 53 0 SDQ_54 0 SDQ_55 0 SDQ_56 SDQ_57 0 SDQ 58 $\sim 0$ SDQ_59 0 SDQ_60 0 SDQ_61 0 SDQ_62 0 SDQ_63 0 SDQ_64 SDQ_65 0 SDQ_66 0 SDQ $67 \sim 0$ SDQ 68 0 SDQ 69 0 SDQ 70 0 SDQ 71 0 SDQ 72 0 SDQ 73 0 SDQ 74 0 SDQ 75 0 SDQ 76 0 (*t1); parent by SDQ_1 0 SDQ_2 0 SDQ_3 0 SDQ_4 0 SDQ_5 SDQ_6 0 SDQ_7 0 SDQ_8 0 SDQ_9 0 SDQ_10 0 SDQ_11 0 SDQ_12 SDQ_13 0 
SDQ_14 0 SDQ_15 0 SDQ_16 0 SDQ_17 0 SDQ 18 0 SDQ_19 SDQ 20 0 SDQ 21 0 SDQ_22 0 SDQ 23 0 SDQ_24 0 SDQ_25 0 SDQ_26 SDQ 27 0 SDQ 28 0 SDQ 29 0 SDQ 30 0 SDQ 31 0 SDQ 32 0 SDQ 33 0 SDQ 34 SDQ 35 0 SDQ 36 0 SDQ 37 0 SDQ 38 0 SDQ 39 0 SDQ_40 0 SDQ_41 0 SDQ_42 SDQ_43 0 SDQ 44 $\sim 0$ SDQ_45 0 SDQ_46 0 SDQ 47 $\sim 0$ SDQ_48 0 SDQ_49 0 SDQ_50 SDQ 51 0 SDQ_52 0 SDQ 53 0 SDQ 54 0 SDQ 55 0 SDQ 56 0 SDQ 57 0 SDQ 58 SDQ 59 0 SDQ 60 0 SDQ 61 0 SDQ 62 0 SDQ 63 0 SDQ 64 0 SDQ 65 0 SDQ_66 SDQ_67 0 SDQ_68 0 SDQ_69 0 SDQ_70 0 SDQ 71 0 SDQ_72 0 SDQ_73 0 SDQ_74 0 SDQ_75 0 SDQ_76 0 (*t1);

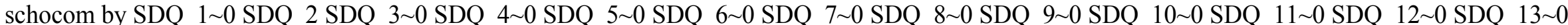
SDQ 14 0 SDQ $15 \sim 0$ SDQ 16 SDQ $17 \sim 0$ SDQ 18 0 SDQ 19 0 SDQ 20 0 SDQ 21 0 SDQ 22 0 SDQ 23 0 SDQ 24 0 SDQ 25 0 SDQ 26 0 SDQ 27 0 SDQ 28 0 SDQ 29 0 SDQ 30 0 SDQ 31 SDQ 32 0 SDQ 33 0 SDQ 34 0 SDQ 35 0 SDQ 36 0 SDQ 37 0 SDQ 38 0 SDQ 39 0 SDQ _40 0 SDQ 41 0 SDQ 42 0 SDQ 43 0 SDQ 44 0 SDQ 45 0 SDQ 46 0 SDQ 47 SDQ 48 0 SDQ 49 0 SDQ 50 0 SDQ 51 0 SDQ 52 0 SDQ 53 0 SDQ 54 0 SDQ 55 0 SDQ 56 0 SDQ 57 0 SDQ 58 0 SDQ 59 0 SDQ 60 0 SDQ 61 0 SDQ 62 0 SDQ 63 SDQ 64 0 SDQ_65 0 SDQ_66 0 SDQ_67 0 SDQ_68 0 SDQ 69 0 SDQ 70 0 SDQ 71 0 SDQ 72 0 SDQ 73 0 SDQ 74 0 SDQ 75 0 SDQ 76 0 (*t1);

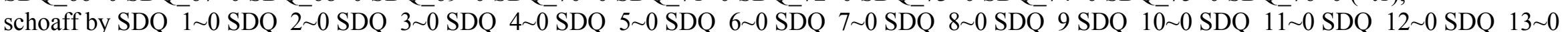
SDQ_14 0 SDQ_15 0 SDQ_16 0 SDQ_17 0 SDQ_18 0 SDQ_19 0 SDQ_20 0 SDQ 21 $\sim 0$ SDQ_22 0 SDQ_23 SDQ_24 0 SDQ_25 0 SDQ_26 0 SDQ_27 0 SDQ_28 0 SDQ_29 0 SDQ_30 0 SDQ_31 0 SDQ_32 0 SDQ_33 0 SDQ_34 0 SDQ_35 0 SDQ_36 0 SDQ_37 0 SDQ_38 0 SDQ_39 SDQ 40 0 SDQ 41 0 SDQ 42 0 SDQ 43 0 SDQ 44 0 SDQ 45 0 SDQ 46 0 SDQ 47 0 SDQ 48 0 SDQ 49 0 SDQ 50 0 SDQ 51 0 SDQ 52 0 SDQ 53 0 SDQ 54 0 SDQ_55 SDQ 56 0 SDQ 57 $\sim 0$ SDQ 58 0 SDQ_59 0 SDQ_60 0 SDQ_61 0 SDQ_62 0 SDQ_63 0 SDQ_64 0 SDQ_65 0 SDQ 66 0 SDQ 67 0 SDQ_68 0 SDQ 69 0 SDQ 70 0 SDQ 71 SDQ 72 0 SDQ 73 0 SDQ 74 0 SDQ 75 0 SDQ 76 0 (*t1);

Germcom by SDQ_1 0 SDQ_2 0 SDQ_3 0 SDQ_4 SDQ 5 0 SDQ_6 0 SDQ_7 0 SDQ_8 0 SDQ_9 0 SDQ_10 0 SDQ_11 0 SDQ 12 0 SDQ_13 0 SDQ 14 0 SDQ 15 0 SDQ_16 0 SDQ_17 0 SDQ 18 SDQ 19 0 SDQ 20 0 SDQ 21 $\sim 0$ SDQ 22 0 SDQ 23 0 SDQ 24 0 SDQ 25 0 SDQ 26 0 SDQ_27 0 SDQ_28 0 SDQ_29 0 SDQ_30 0 SDQ_31 0 SDQ_32 0 SDQ_33 SDQ_34 0 SDQ_35 0 SDQ_36 0 SDQ_37 0 SDQ_38 0 SDQ_39 0 SDQ 40 0 SDQ 41 0 SDQ 42 0 SDQ 43 0 SDQ 44 0 SDQ 45 0 SDQ 46 0 SDQ 47 0 SDQ 48 0 SDQ 49 SDQ 50 0 SDQ 51 0 SDQ 52 0 SDQ_53 0 SDQ_54 0 SDQ_55 0 SDQ_56 0 SDQ_57 0 SDQ_58 0 SDQ_59 0 SDQ_60 0 SDQ_61 0 SDQ_62 0 SDQ_63 0 SDQ_64 0 SDQ_65 0 SDQ 66 0 SDQ 67 0 SDQ 68 0 SDQ 69 0 SDQ 70 0 SDQ 71 0 SDQ 72 0 SDQ 73 SDQ 74 0 SDQ 75 0 SDQ 76 0 $(* 11)$; Germaff by SDQ_1 0 SDQ 2 $\sim 0$ SDQ 3 3 0 SDQ_4 0 SDQ 5 0 SDQ_6 0 SDQ 7 0 SDQ_8 0 SDQ_9 0 SDQ_10 0 SDQ_11 SDQ_12 0 SDQ_13 0 SDQ 14 0 SDQ 15 0 SDQ 16 0 SDQ 17 0 SDQ 18 0 SDQ 19 0 SDQ 20 0 SDQ 21 0 SDQ 22 0 SDQ 23 0 SDQ $24 \sim 0$ SDQ 25 SDQ $26 \sim 0$ SDQ_27 0 SDQ_28 0 SDQ_29 0 SDQ 30 0 SDQ_31 0 SDQ_32 0 SDQ_33 0 SDQ_34 0 SDQ_35 0 SDQ_36 0 SDQ_37 0 SDQ 38 0 SDQ_39 0 SDQ 40 0 SDQ 41 SDQ 42 0 SDQ 43 0 SDQ 44 0 SDQ 45 0 SDQ 46 0 SDQ 47 $\sim 0$ SDQ 48 0 SDQ 49 0 SDQ 50 0 SDQ 51 0 SDQ 52 0 SDQ_53 0 SDQ_54 0 SDQ 55 $\sim 0$ SDQ_56 0 SDQ 57 SDQ_58 0 SDQ_59 0 SDQ_60 0 SDQ_61 0 SDQ_62 0 SDQ_63 0 SDQ_64 0 SDQ_65 SDQ 66 0 SDQ 67 0 SDQ 68 0 SDQ 69 0 SDQ 70 0 SDQ 71 0 SDQ 72 0 SDQ 73 0 SDQ 74 0 SDQ $75 \sim 0$ SDQ $76 \sim 0$ (*t1); mathcom by SDQ_1 0 SDQ 2 2 0 SDQ 3 0 SDQ 4 0 SDQ 5 0 SDQ 6 6 0 SDQ 7 0 SDQ 8 0 SDQ 9 9 SDQ 10 0 SDQ 11 0 SDQ 12 0 SDQ_13 SDQ 14 0 SDQ 15 0 SDQ 16 0 SDQ 17 0 SDQ 18 0 SDQ 19 0 SDQ 20 0 SDQ 21 0 SDQ 22 0 SDQ 23 0 SDQ 24 0 SDQ 25 0 SDQ 26 0 SDQ 27 SDQ_28 0 SDQ 29 $\sim 0$ SDQ_30 0 SDQ_31 0 SDQ 32 0 SDQ_33 0 SDQ_34 0 SDQ_35 0 SDQ_36 0 SDQ_37 0 SDQ_38 0 SDQ_39 0 SDQ 40 0 SDQ 41 0 SDQ 42 0 SDQ 43 SDQ 44 0 SDQ 45 0 SDQ 46 0 SDQ 47 0 SDQ 48 0 SDQ 49 0 SDQ 50 0 SDQ 51 0 SDQ 52 0 SDQ 53 0 SDQ 54 0 SDQ 55 0 SDQ 56 0 SDQ 57 0 SDQ 58 0 SDQ 59 SDQ 60 0 SDQ 61 0 SDQ 62 0 SDQ 63 0 SDQ 64 0 SDQ 65 0 SDQ_66 0 SDQ_67 0 SDQ_68 0 SDQ_69 0 SDQ_70 0 SDQ_71 0 SDQ_72 0 SDQ_73 0 SDQ_74 0 SDQ_75 SDQ_76 0 (*t1); 
mathaff by SDQ_1 0 SDQ 2 $0 \mathrm{SDQ}$ 3 0 SDQ 4 4 SDQ 5 5 SDQ 6 SDQ 7 0 SDQ 8 0 SDQ 9 9 SDQ_10 0 SDQ 11 0 SDQ 12 0 SDQ 13 0 SDQ 14 0 SDQ 15 0 SDQ 16 0 SDQ 17 0 SDQ 18 0 SDQ 19 0 SDQ 20 SDQ 21 0 SDQ 22 0 SDQ 23 0 SDQ 24 0 SDQ 25 0 SDQ 26 0 SDQ_27 0 SDQ_28 0 SDQ_29 0 SDQ_30 0 SDQ_31 0 SDQ_32 0 SDQ_33 0 SDQ_34 0 SDQ_35 SDQ_36 0 SDQ_37 0 SDQ_38 0 SDQ_39 0 SDQ 40 0 SDQ 41 0 SDQ 42 0 SDQ 43 0 SDQ 44 0 SDQ 45 0 SDQ 46 0 SDQ 47 0 SDQ 48 0 SDQ 49 0 SDQ 50 0 SDQ 51 SDQ 52 0 SDQ_53 0 SDQ_54 0 SDQ_55 0 SDQ_56 0 SDQ 57 0 SDQ_58 0 SDQ_59 0 SDQ_60 0 SDQ_61 0 SDQ_62 0 SDQ_63 0 SDQ_64 0 SDQ_65 0 SDQ 66 0 SDQ 67 0 SDQ 68 SDQ 69 0 SDQ_70 0 SDQ 71 0 SDQ 72 0 SDQ_73 0 SDQ 74 0 SDQ_75 0 SDQ 76 0 (*t1);

! Method Factor (negative items)

MF BY SDQ $30 *$ (MF1)

SDQ_17 SDQ_12 SDQ_21 SDQ_47 SDQ_23 SDQ_33 SDQ_65 SDQ_75 SDQ_6 SDQ_37 SDQ_61 (MF2-MF12);

MF@1; [MF@0];

MFWITH GLOBAL@0 esteem@0 peer@0 appear@0 phy@0 parent@0 schocom@0

schoaff@0 Germcom@0 Germaff@0 MathAff@0 MathCom@0;

! correlated uniquenesses between parallel worded items

SDQ_11 with SDQ 51 SDQ 71; SDQ 51 with SDQ 71;

SDQ_25 with SDQ_35 SDQ_39; SDQ_35 with SDQ _39;

SDQ_41 with SDQ_68 SDQ_9; SDQ_68 with SDQ 9;

SDQ 57 with SDQ 20 SDQ_55; SDQ 20 with SDQ 55;

SDQ_23 with SDQ_6 SDQ_65; SDQ_6 with SDQ_65;

SDQ 4 with SDQ 27 SDQ 16; SDQ 27 with SDQ 16;

SDQ_18 with SDQ_59 SDQ_2; SDQ_59 with SDQ_2;

SDQ 49 with SDQ 13 SDQ 63; SDQ 13 with SDQ 63;

SDQ_73 with SDQ_43 SDQ_31; SDQ_43 with SDQ_31;

SDQ 47 with SDQ 75 SDQ 33; SDQ 75 with SDQ 33;

SDQ_1 (1); SDQ_2 (2); SDQ_3 (3); SDQ_4 (4); SDQ_5 (5); SDQ_6 (6); SDQ 7 (7); SDQ 8 (8);

SDQ 9 (9); SDQ 10 (10); SDQ 11 (11); SDQ 12 (12);

SDQ_13 (13); SDQ_14 (14); SDQ_15 (15); SDQ_16 (16);

SDQ 17 (17); SDQ 18 (18); SDQ 19 (19); SDQ 20 (20);

SDQ_21 (21); SDQ_22 (22); SDQ_23 (23); SDQ_24 (24);

SDQ 25 (25); SDQ 26 (26); SDQ 27 (27); SDQ 28 (28);

SDQ_29 (29); SDQ_30 (30); SDQ_31 (31); SDQ_32 (32);

SDQ 33 (33); SDQ 34 (34); SDQ 35 (35); SDQ 36 (36):

SDQ_37 (37); SDQ_38 (38); SDQ_39 (39); SDQ_40 (40);

SDQ 41 (41); SDQ 42 (42); SDQ 43 (43); SDQ 44 (44);

SDQ 45 (45); SDQ 46 (46); SDQ 47 (47); SDQ 48 (48);

SDQ_49 (49); SDQ_50 (50); SDQ_51 (51); SDQ_52 (52); 
SDQ_53 (53); SDQ_54 (54); SDQ_55 (55); SDQ_56 (56); SDQ 57 (57); SDQ 58 (58); SDQ 59 (59); SDQ 60 (60); SDQ_61 (61); SDQ_62 (62); SDQ_63 (63); SDQ_64 (64); SDQ 65 (65); SDQ 66 (66); SDQ 67 (67); SDQ 68 (68); SDQ_69 (69); SDQ_70 (70); SDQ_71 (71); SDQ_72 (72); SDQ 73 (73); SDQ 74 (74); SDQ 75 (75); SDQ 76 (76); model female:

! Method Factor (negative items)

MF BY SDQ 30* (MF1)

SDQ 17 SDQ 12 SDQ 21 SDQ 47 SDQ 23 SDQ 33 SDQ 65 SDQ 75 SDQ 6 SDQ 37 SDQ 61 (MF2-MF12);

$\mathrm{MF}^{*} ;[\mathrm{MF} *]$;

MF WITH GLOBAL@0 esteem@0 peer@0 appear@0 phy@0 parent@0 schocom@0

schoaff@0.Germcom@0 Germaff@0 MathAff@0 MathCom@0;

! correlated uniquenesses between parallel worded items

SDQ_11 with SDQ_51 SDQ_71; SDQ_51 with SDQ_71;

SDQ 25 with SDQ 35 SDQ 39; SDQ 35 with SDQ 39 ;

SDQ 41 with SDQ 68 SDQ 9; SDQ 68 with SDQ 9;

SDQ_57 with SDQ_20 SDQ_55; SDQ_20 with SDQ 55;

SDQ 23 with SDQ 6 SDQ 65; SDQ 6 with SDQ 65;

SDQ_4 with SDQ 27 SDQ_16; SDQ_27 with SDQ_16;

SDQ 18 with SDQ 59 SDQ 2; SDQ 59 with SDQ 2;

SDQ_49 with SDQ_13 SDQ_63; SDQ_13 with SDQ_63;

SDQ 73 with SDQ 43 SDQ 31; SDQ 43 with SDQ 31;

SDQ_47 with SDQ_75 SDQ_33; SDQ_75 with SDQ_33;

SDQ 1 (1); SDQ 2 (2); SDQ 3 (3); SDQ 4 (4); SDQ 5 (5); SDQ 6 (6); SDQ 7 (7); SDQ 8 (8);

SDQ_9 (9); SDQ_10 (10); SDQ_11 (11); SDQ_12 (12);

SDQ 13 (13); SDQ 14 (14); SDQ 15 (15); SDQ 16 (16);

SDQ_17 (17); SDQ_18 (18); SDQ_19 (19); SDQ_20 (20);

SDQ 21 (21); SDQ 22 (22); SDQ 23 (23); SDQ 24 (24);

SDQ_25 (25); SDQ_26 (26); SDQ_27 (27); SDQ_28 (28);

SDQ 29 (29); SDQ 30 (30); SDQ 31 (31); SDQ 32 (32);

SDQ 33 (33); SDQ_34 (34); SDQ_35 (35); SDQ 36 (36):

SDQ_37 (37); SDQ_38 (38); SDQ_39 (39); SDQ 40 (40);

SDQ 41 (41); SDQ 42 (42); SDQ 43 (43); SDQ 44 (44);

SDQ 45 (45); SDQ 46 (46); SDQ 47 (47); SDQ 48 (48); 
SDQ_49 (49); SDQ_50 (50); SDQ_51 (51); SDQ_52 (52);

SDQ 53 (53); SDQ 54 (54); SDQ 55 (55); SDQ 56 (56);

SDQ_57 (57); SDQ_58 (58); SDQ_59 (59); SDQ_60 (60);

SDQ 61 (61); SDQ 62 (62); SDQ 63 (63); SDQ 64 (64);

SDQ_65 (65); SDQ_66 (66); SDQ_67 (67); SDQ_68 (68);

SDQ 69 (69); SDQ 70 (70); SDQ 71 (71); SDQ 72 (72);

SDQ_73 (73); SDQ_74 (74); SDQ_75 (75); SDQ_76 (76); 
Title: Multiple Group Correlated Uniquenesses - CUs (Loadings, Intercepts, Uniquenesses, CUs) Invariance Model of the SDQ-I (Real Data) ! [...]Model section only

GLOBAL BY SDQ_1 SDQ_2 SDQ_3 SDQ 4 SDQ_5 SDQ_6 SDQ 7 SDQ 8 SDQ 9 SDQ 10

SDQ_11 SDQ_12 SDQ_13 SDQ_14 SDQ_15 SDQ_16 SDQ_17 SDQ_18 SDQ_19 SDQ_20

SDQ_21 SDQ_22 SDQ_23 SDQ_24 SDQ_25 SDQ_26 SDQ_27 SDQ_28 SDQ_29 SDQ_30

SDQ 31 SDQ 32 SDQ 33 SDQ 34 SDQ 35 SDQ 36 SDQ 37 SDQ 38 SDQ 39 SDQ 40

SDQ_41 SDQ_42 SDQ_43 SDQ_44 SDQ_45 SDQ_46 SDQ_47 SDQ_48 SDQ_49 SDQ_50

SDQ 51 SDQ 52 SDQ 53 SDQ 54 SDQ 55 SDQ 56 SDQ 57 SDQ 58 SDQ 59 SDQ 60

SDQ_61 SDQ_62 SDQ_63 SDQ_64 SDQ_65 SDQ_66 SDQ_67 SDQ_68 SDQ_69 SDQ_70

SDQ 71 SDQ 72 SDQ 73 SDQ 74 SDQ 75 SDQ $76(* t 1)$

esteem by SDQ_1 0 SDQ 2 0 SDQ 3 0 SDQ 4 0 SDQ 5 0 SDQ_6 0 SDQ 7 7 0 SDQ_8 0 SDQ_9 0 SDQ_10 0 SDQ_11 0 SDQ_12 0 SDQ_13 0 SDQ 14 0 SDQ 15 0 SDQ 16 0 SDQ 17 0 SDQ 18 0 SDQ 19 0 SDQ 20 0 SDQ 21 0 SDQ 22 0 SDQ 23 0 SDQ 24 0 SDQ 25 0 SDQ 26 0 SDQ_27 0 SDQ_28 0 SDQ_29 SDQ_30 0 SDQ_31 0 SDQ_32 0 SDQ_33 0 SDQ_34 0 SDQ_35 0 SDQ_36 0 SDQ_37 SDQ_38 0 SDQ_39 0

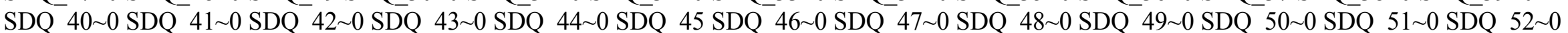
SDQ_53 SDQ 54 0 SDQ 55 0 SDQ_56 0 SDQ 57 0 SDQ 58 0 SDQ_59 0 SDQ_60 0 SDQ_61 SDQ_62 0 SDQ_63 0 SDQ_64 0 SDQ_65 0 SDQ 66 0 SDQ 67 SDQ 68 0 SDQ 69 0 SDQ 70 SDQ 71 0 SDQ 72 SDQ 73 0 SDQ 74 SDQ 75 0 SDQ $76(* 1)$;

peer by SDQ 1 0 SDQ 2 2 0 SDQ 3 0 SDQ 4 0 SDQ 5 0 SDQ 6 6 SDQ 7 SDQ 8 0 SDQ 9 0 SDQ 10 0 SDQ 11 0 SDQ 12 0 SDQ 13 0 SDQ_14 SDQ_15 0 SDQ_16 0 SDQ_17 0 SDQ_18 0 SDQ_19 0 SDQ 20 0 SDQ_21 SDQ_22 0 SDQ_23 0 SDQ_24 0 SDQ_25 0 SDQ_26 0 SDQ 27 0 SDQ 28 SDQ 29 0 SDQ 30 0 SDQ 31 0 SDQ 32 0 SDQ 33 0 SDQ 34 0 SDQ 35 0 SDQ 36 SDQ 37 0 SDQ 38 0 SDQ 39 0 SDQ $40 \sim 0 \mathrm{SDQ}$ 41 0 SDQ 42 0 SDQ 43 0 SDQ 44 SDQ 45 0 SDQ_46 0 SDQ 47 0 SDQ 48 0 SDQ 49 0 SDQ 50 0 SDQ 51 0 SDQ 52 SDQ 53 0 SDQ 54 0 SDQ 55 0 SDQ 56 0 SDQ 57 0 SDQ 58 0 SDQ 59 0 SDQ 60 SDQ 61 0 SDQ 62 0 SDQ 63 0 SDQ 64 0 SDQ 65 0 SDQ 66 0 SDQ 67 0 SDQ 68 0 SDQ 69 SDQ 70 0 SDQ 71 0 SDQ 72 0 SDQ 73 0 SDQ 74 0 SDQ 75 0 SDQ 76 0 $(* 11)$;

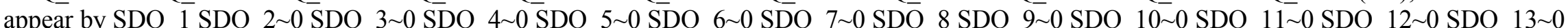
SDQ_14 0 SDQ 15 SDQ 16 0 SDQ 17 0 SDQ 18 0 SDQ_19 0 SDQ 20 0 SDQ 21 $\sim 0$ SDQ 22 SDQ 23 0 SDQ_24 0 SDQ 25 0 SDQ_26 0 SDQ 27 0 SDQ 28 0 SDQ 29 0 SDQ 30 SDQ 31 0 SDQ 32 0 SDQ 33 0 SDQ 34 0 SDQ 35 0 SDQ 36 0 SDQ 37 0 SDQ 38 SDQ 39 0 SDQ $40 \sim 0 \mathrm{SDQ}$ 41 0 SDQ_42 0 SDQ_43 0 SDQ 44 0 SDQ 45 0 SDQ 46 SDQ 47 0 SDQ_48 0 SDQ_49 0 SDQ_50 0 SDQ_51 0 SDQ_52 0 SDQ 53 0 SDQ 54 SDQ 55 0 SDQ 56 0 SDQ 57 0 SDQ 58 0 SDQ 59 0 SDQ 60 0 SDQ 61 0 SDQ 62 SDQ 63 0 SDQ $64 \sim 0$ SDQ $65 \sim 0$ SDQ $66 \sim 0$ SDQ 67 0 SDQ 68 0 SDQ 69 0 SDQ 70 0 SDQ 71 0 SDQ 72 0 SDQ 73 0 SDQ 74 0 SDQ 75 0 SDQ 76 0 (*t1);

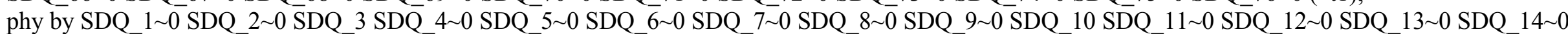
SDQ_15 0 SDQ_16 0 SDQ_17 SDQ_18 0 SDQ 19 0 SDQ_20 0 SDQ_21 0 SDQ 22 0 SDQ_23 0 SDQ_24 SDQ_25 0 SDQ 26 0 SDQ_27 0 SDQ 28 0 SDQ 29 0 SDQ 30 0 SDQ 31 0 SDQ $32 \mathrm{SDQ}$ 33 0 SDQ 34 0 SDQ 35 0 SDQ 36 0 SDQ 37 0 SDQ 38 0 SDQ 39 0 SDQ 40 SDQ_41 0 SDQ_42 0 SDQ_43 0 SDQ 44 0 SDQ_45 0 SDQ 46 0 SDQ 47 0 SDQ 48 SDQ_49 0 SDQ_50 0 SDQ_51 0 SDQ_52 0 SDQ 53 0 SDQ_54 0 SDQ_55 0 SDQ_56 SDQ_57 0 SDQ 58 $\sim 0$ SDQ_59 0 SDQ_60 0 SDQ_61 0 SDQ_62 0 SDQ_63 0 SDQ_64 SDQ_65 0 SDQ_66 0 SDQ $67 \sim 0$ SDQ 68 0 SDQ 69 0 SDQ 70 0 SDQ 71 0 SDQ 72 0 SDQ 73 0 SDQ 74 0 SDQ 75 0 SDQ 76 0 (*t1); parent by SDQ_1 0 SDQ_2 0 SDQ_3 0 SDQ_4 0 SDQ_5 SDQ_6 0 SDQ_7 0 SDQ_8 0 SDQ_9 0 SDQ_10 0 SDQ_11 0 SDQ_12 SDQ_13 0 
SDQ_14 0 SDQ_15 0 SDQ_16 0 SDQ_17 0 SDQ_18 0 SDQ_19 SDQ_20 0 SDQ_21 0 SDQ_22 0 SDQ_23 0 SDQ_24 0 SDQ 25 0 SDQ_26 SDQ 27 0 SDQ 28 0 SDQ 29 0 SDQ 30 0 SDQ 31 0 SDQ 32 0 SDQ 33 0 SDQ 34 SDQ 35 0 SDQ 36 0 SDQ 37 0 SDQ 38 0 SDQ 39 0 SDQ_40 0 SDQ_41 0 SDQ_42 SDQ_43 0 SDQ 44 $\sim 0$ SDQ_45 0 SDQ 46 0 SDQ_47 $\sim 0$ SDQ_48 0 SDQ_49 0 SDQ_50 SDQ 51 0 SDQ_52 0 SDQ 53 0 SDQ 54 0 SDQ 55 0 SDQ 56 0 SDQ 57 0 SDQ 58 SDQ 59 0 SDQ 60 0 SDQ 61 0 SDQ 62 0 SDQ 63 0 SDQ 64 0 SDQ 65 0 SDQ_66 SDQ_67 0 SDQ_68 0 SDQ_69 0 SDQ_70 0 SDQ 71 0 SDQ_72 0 SDQ_73 0 SDQ_74 0 SDQ_75 0 SDQ_76 0 (*t1);

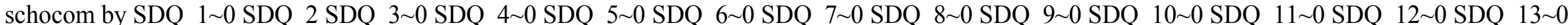
SDQ 14 0 SDQ $15 \sim 0$ SDQ 16 SDQ $17 \sim 0$ SDQ 18 0 SDQ 19 0 SDQ 20 0 SDQ 21 0 SDQ 22 0 SDQ 23 0 SDQ 24 0 SDQ 25 0 SDQ 26 0 SDQ 27 0 SDQ 28 0 SDQ 29 0 SDQ 30 0 SDQ 31 SDQ 32 0 SDQ 33 0 SDQ 34 0 SDQ 35 0 SDQ 36 0 SDQ 37 0 SDQ 38 0 SDQ 39 0 SDQ _40 0 SDQ 41 0 SDQ 42 0 SDQ 43 0 SDQ 44 0 SDQ 45 0 SDQ 46 0 SDQ 47 SDQ 48 0 SDQ 49 0 SDQ 50 0 SDQ 51 0 SDQ 52 0 SDQ 53 0 SDQ 54 0 SDQ 55 0 SDQ 56 0 SDQ 57 0 SDQ 58 0 SDQ 59 0 SDQ 60 0 SDQ 61 0 SDQ 62 0 SDQ_63 SDQ 64 0 SDQ_65 0 SDQ_66 0 SDQ_67 0 SDQ_68 0 SDQ 69 0 SDQ 70 0 SDQ 71 0 SDQ 72 0 SDQ 73 0 SDQ 74 0 SDQ 75 0 SDQ 76 0 (*t1); schoaff by SDQ 1 $\sim 0$ SDQ 2 0 SDQ 3 0 SDQ 4 0 SDQ 5 0 SDQ $6 \sim 0$ SDQ $7 \sim 0$ SDQ $8 \sim 0$ SDQ 9 SDQ $10 \sim 0$ SDQ $11 \sim 0$ SDQ $12 \sim 0$ SDQ $13 \sim 0$ SDQ_14 0 SDQ_15 0 SDQ_16 0 SDQ_17 0 SDQ_18 0 SDQ_19 0 SDQ_20 0 SDQ 21 $\sim 0$ SDQ_22 0 SDQ_23 SDQ_24 0 SDQ_25 0 SDQ_26 0 SDQ_27 0 SDQ_28 0 SDQ_29 0 SDQ_30 0 SDQ_31 0 SDQ_32 0 SDQ_33 0 SDQ_34 0 SDQ_35 0 SDQ_36 0 SDQ_37 0 SDQ_38 0 SDQ 39 SDQ 40 0 SDQ 41 0 SDQ 42 0 SDQ 43 0 SDQ 44 0 SDQ 45 0 SDQ 46 0 SDQ 47 0 SDQ 48 0 SDQ 49 0 SDQ 50 0 SDQ 51 0 SDQ 52 0 SDQ_53 0 SDQ_54 0 SDQ_55 SDQ_56 0 SDQ_57 0 SDQ_58 0 SDQ_59 0 SDQ_60 $\sim 0$ SDQ_61 0 SDQ_62 0 SDQ_63 0 SDQ_64 0 SDQ_65 0 SDQ 66 0 SDQ 67 0 SDQ_68 0 SDQ 69 0 SDQ 70 0 SDQ 71 SDQ 72 0 SDQ 73 0 SDQ 74 0 SDQ 75 0 SDQ 76 0 (*t1);

Germcom by SDQ_1 0 SDQ_2 0 SDQ_3 0 SDQ_4 SDQ 5 0 SDQ_6 0 SDQ_7 0 SDQ_8 0 SDQ_9 0 SDQ_10 0 SDQ_11 0 SDQ 12 0 SDQ_13 0 SDQ 14 0 SDQ 15 0 SDQ 16 0 SDQ 17 0 SDQ 18 SDQ 19 0 SDQ 20 0 SDQ 21 0 SDQ 22 0 SDQ 23 0 SDQ 24 0 SDQ 25 0 SDQ 26 0 SDQ_27 0 SDQ_28 0 SDQ_29 0 SDQ_30 0 SDQ_31 0 SDQ_32 0 SDQ_33 SDQ_34 0 SDQ_35 0 SDQ_36 0 SDQ_37 0 SDQ_38 0 SDQ_39 0 SDQ 40 0 SDQ 41 0 SDQ 42 0 SDQ 43 0 SDQ 44 0 SDQ 45 0 SDQ 46 0 SDQ 47 0 SDQ 48 0 SDQ 49 SDQ 50 0 SDQ 51 0 SDQ 52 0 SDQ_53 0 SDQ_54 0 SDQ_55 0 SDQ_56 0 SDQ_57 0 SDQ_58 0 SDQ_59 0 SDQ_60 0 SDQ_61 0 SDQ_62 0 SDQ_63 0 SDQ_64 0 SDQ_65 0 SDQ $66 \sim 0$ SDQ 67 0 SDQ 68 0 SDQ 69 0 SDQ 70 0 SDQ 71 0 SDQ $72 \sim 0$ SDQ 73 SDQ 74 0 SDQ 75 0 SDQ 76 0 $(* 11)$; Germaff by SDQ_1 0 SDQ 2 $\sim 0$ SDQ 3 $\sim 0$ SDQ_4 0 SDQ 5 0 SDQ_6 0 SDQ 7 0 SDQ_8 0 SDQ_9 0 SDQ_10 0 SDQ_11 SDQ_12 0 SDQ_13 0 SDQ 14 0 SDQ 15 0 SDQ 16 0 SDQ 17 0 SDQ 18 0 SDQ 19 0 SDQ 20 0 SDQ 21 0 SDQ 22 0 SDQ 23 0 SDQ 24 0 SDQ 25 SDQ $26 \sim 0$ SDQ_27 0 SDQ_28 0 SDQ_29 0 SDQ 30 0 SDQ_31 0 SDQ_32 0 SDQ_33 0 SDQ_34 0 SDQ_35 0 SDQ_36 0 SDQ_37 0 SDQ 38 0 SDQ_39 0

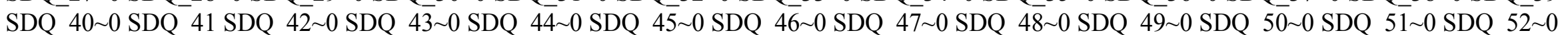
SDQ_53 0 SDQ_54 0 SDQ 55 $\sim 0$ SDQ_56 0 SDQ 57 SDQ_58 0 SDQ_59 0 SDQ_60 0 SDQ_61 0 SDQ_62 0 SDQ_63 0 SDQ_64 0 SDQ_65 SDQ 66 0 SDQ 67 0 SDQ 68 0 SDQ 69 0 SDQ 70 0 SDQ 71 0 SDQ 72 0 SDQ 73 0 SDQ 74 0 SDQ 75 0 SDQ 76 0 (*t1); mathcom by SDQ_1 0 SDQ_2 0 SDQ_3 0 SDQ 4 0 SDQ_5 0 SDQ 6 6 SDQ_7 0 SDQ 8 0 SDQ_9 0 SDQ_10 0 SDQ_11 0 SDQ_12 0 SDQ_13

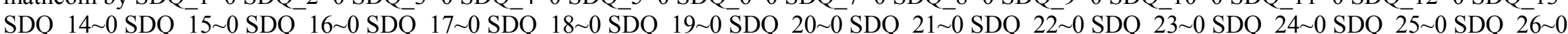
SDQ 27 SDQ 28 0 SDQ 29 0 SDQ 30 0 SDQ 31 0 SDQ 32 0 SDQ 33 0 SDQ 34 0 SDQ 35 0 SDQ 36 0 SDQ 37 0 SDQ 38 0 SDQ 39 0 SDQ 40 0 SDQ 41 0 SDQ 42 0 SDQ 43 SDQ 44 0 SDQ 45 0 SDQ 46 0 SDQ 47 0 SDQ 48 0 SDQ 49 0 SDQ 50 0 SDQ 51 0 SDQ 52 0 SDQ 53 0 SDQ 54 0 SDQ 55 0 SDQ 56 0 SDQ 57 0 SDQ 58 0 SDQ 59 SDQ 60 0 SDQ 61 0 SDQ 62 0 SDQ 63 0 SDQ 64 0 SDQ 65 0 SDQ_66 0 SDQ_67 0 SDQ_68 0 SDQ_69 0 SDQ_70 0 SDQ_71 0 SDQ_72 0 SDQ_73 0 SDQ_74 0 SDQ_75 SDQ_76 0 (*t1); 
mathaff by SDQ_1 0 SDQ 2 0 SDQ_3 0 SDQ 4 0 SDQ_5 0 SDQ 6 SDQ_7 0 SDQ 8 0 SDQ_9 0 SDQ_10 0 SDQ_11 0 SDQ_12 0 SDQ_13 0 SDQ 14 0 SDQ 15 0 SDQ 16 0 SDQ 17 0 SDQ 18 0 SDQ 19 0 SDQ 20 SDQ 21 0 SDQ 22 0 SDQ 23 0 SDQ 24 0 SDQ 25 0 SDQ 26 0 SDQ_27 0 SDQ_28 0 SDQ_29 0 SDQ_30 0 SDQ_31 0 SDQ_32 0 SDQ_33 0 SDQ_34 0 SDQ_35 SDQ_36 0 SDQ_37 0 SDQ_38 0 SDQ_39 0 SDQ 40 0 SDQ 41 0 SDQ 42 0 SDQ 43 0 SDQ 44 0 SDQ 45 0 SDQ 46 0 SDQ 47 0 SDQ 48 0 SDQ 49 0 SDQ 50 0 SDQ 51 SDQ 52 0 SDQ_53 0 SDQ_54 0 SDQ_55 0 SDQ_56 0 SDQ 57 0 SDQ_58 0 SDQ_59 0 SDQ_60 0 SDQ_61 0 SDQ_62 0 SDQ_63 0 SDQ_64 0 SDQ_65 0 SDQ 66 0 SDQ 67 0 SDQ 68 SDQ 69 0 SDQ_70 0 SDQ 71 0 SDQ 72 0 SDQ_73 0 SDQ 74 0 SDQ_75 0 SDQ 76 0 (*t1);

! Method Factor (negative items)

MF BY SDQ $30 *$ (MF1)

SDQ_17 SDQ_12 SDQ_21 SDQ_47 SDQ_23 SDQ_33 SDQ_65 SDQ_75 SDQ_6 SDQ_37 SDQ_61 (MF2-MF12);

MF@1; [MF@0];

MFWITH GLOBAL@0 esteem@0 peer@0 appear@0 phy@0 parent@0 schocom@0 schoaff@0 Germcom@0 Germaff@0 MathAff@0 MathCom@0;

! correlated uniquenesses between parallel worded items

SDQ 11 with SDQ 51 (cu1); SDQ 11 with SDQ 71 (cu2); SDQ 51 with SDQ 71 (cu3);

SDQ_25 with SDQ_35 (cu4); SDQ_25 with SDQ 39 (cu5); SDQ 35 with SDQ_39 (cu6);

SDQ_41 with SDQ_68 (cu7); SDQ 41 with SDQ_9 (cu8); SDQ_68 with SDQ 9 (cu9);

SDQ 57 with SDQ 20 (cu10); SDQ 57 with SDQ 55 (cu11); SDQ 20 with SDQ 55 (cu12);

SDQ_23 with SDQ_6 (cu13); SDQ 23 with SDQ_65 (cu14); SDQ 6 with SDQ 65 (cu15);

SDQ 4 with SDQ 27 (cu16); SDQ 4 with SDQ 16 (cu17); SDQ 27 with SDQ 16 (cu18);

SDQ_18 with SDQ 59 (cu19); SDQ_18 with SDQ_2 (cu20); SDQ 59 with SDQ 2 (cu21);

SDQ 49 with SDQ 13 (cu22); SDQ 49 with SDQ 63 (cu23); SDQ 13 with SDQ 63 (cu24);

SDQ_73 with SDQ_43 (cu25); SDQ_73 with SDQ_31 (cu26); SDQ_43 with SDQ_31 (cu27);

SDQ 47 with SDQ 75 (cu28); SDQ 47 with SDQ 33 (cu29); SDQ 75 with SDQ 33 (cu30);

SDQ_1 (1); SDQ_2 (2); SDQ_3 (3); SDQ_4 (4); SDQ_5 (5); SDQ_6 (6); SDQ 7 (7); SDQ_8 (8);

SDQ 9 (9); SDQ 10 (10); SDQ 11 (11); SDQ 12 (12);

SDQ_13 (13); SDQ_14 (14); SDQ_15 (15); SDQ_16 (16);

SDQ 17 (17); SDQ 18 (18); SDQ 19 (19); SDQ 20 (20);

SDQ_21 (21); SDQ_22 (22); SDQ_23 (23); SDQ_24 (24);

SDQ 25 (25); SDQ 26 (26); SDQ 27 (27); SDQ 28 (28);

SDQ_29 (29); SDQ_30 (30); SDQ_31 (31); SDQ_32 (32);

SDQ 33 (33); SDQ 34 (34); SDQ 35 (35); SDQ 36 (36):

SDQ_37 (37); SDQ_38 (38); SDQ_39 (39); SDQ_40 (40);

SDQ 41 (41); SDQ 42 (42); SDQ 43 (43); SDQ 44 (44);

SDQ 45 (45); SDQ 46 (46); SDQ 47 (47); SDQ 48 (48);

SDQ_49 (49); SDQ 50 (50); SDQ_51 (51); SDQ_52 (52); 
SDQ_53 (53); SDQ_54 (54); SDQ_55 (55); SDQ_56 (56); SDQ 57 (57); SDQ 58 (58); SDQ 59 (59); SDQ 60 (60); SDQ_61 (61); SDQ_62 (62); SDQ_63 (63); SDQ_64 (64); SDQ 65 (65); SDQ 66 (66); SDQ 67 (67); SDQ 68 (68); SDQ_69 (69); SDQ_70 (70); SDQ_71 (71); SDQ_72 (72); SDQ 73 (73); SDQ 74 (74); SDQ 75 (75); SDQ 76 (76); model female:

! Method Factor (negative items)

MF BY SDQ 30* (MF1)

SDQ 17 SDQ 12 SDQ 21 SDQ 47 SDQ 23 SDQ 33 SDQ 65 SDQ 75 SDQ 6 SDQ 37 SDQ 61 (MF2-MF12);

$\mathrm{MF}^{*} ;[\mathrm{MF} *]$;

MFWITH GLOBAL@0 esteem@0 peer@0 appear@0 phy@0 parent@0 schocom@0

schoaff@0.Germcom@0 Germaff@0 MathAff@0 MathCom@0;

! correlated uniquenesses between parallel worded items

SDQ_11 with SDQ_51 (cu1); SDQ_11 with SDQ 71 (cu2); SDQ 51 with SDQ 71 (cu3);

SDQ 25 with SDQ 35 (cu4); SDQ 25 with SDQ 39 (cu5); SDQ 35 with SDQ 39 (cu6);

SDQ 41 with SDQ 68 (cu7); SDQ 41 with SDQ 9 (cu8); SDQ 68 with SDQ 9 (cu9);

SDQ 57 with SDQ 20 (cu10); SDQ 57 with SDQ 55 (cu11); SDQ 20 with SDQ 55 (cu12);

SDQ 23 with SDQ 6 (cu13); SDQ 23 with SDQ 65 (cu14); SDQ 6 with SDQ 65 (cu15);

SDQ 4 with SDQ 27 (cu16); SDQ 4 with SDQ_16 (cu17); SDQ 27 with SDQ 16 (cu18);

SDQ 18 with SDQ 59 (cu19); SDQ 18 with SDQ 2 (cu20); SDQ 59 with SDQ 2 (cu21);

SDQ_49 with SDQ_13 (cu22); SDQ_49 with SDQ_63 (cu23); SDQ_13 with SDQ_63 (cu24);

SDQ 73 with SDQ 43 (cu25); SDQ 73 with SDQ 31 (cu26); SDQ 43 with SDQ 31 (cu27);

SDQ_47 with SDQ_75 (cu28); SDQ_47 with SDQ_33 (cu29); SDQ_75 with SDQ_33 (cu30);

SDQ 1 (1); SDQ 2 (2); SDQ 3 (3); SDQ 4 (4); SDQ 5 (5); SDQ 6 (6); SDQ 7 (7); SDQ 8 (8);

SDQ_9 (9); SDQ_10 (10); SDQ_11 (11); SDQ_12 (12);

SDQ 13 (13); SDQ 14 (14); SDQ 15 (15); SDQ 16 (16);

SDQ_17 (17); SDQ_18 (18); SDQ_19 (19); SDQ_20 (20);

SDQ 21 (21); SDQ 22 (22); SDQ 23 (23); SDQ 24 (24);

SDQ_25 (25); SDQ_26 (26); SDQ_27 (27); SDQ_28 (28);

SDQ 29 (29); SDQ 30 (30); SDQ 31 (31); SDQ 32 (32);

SDQ 33 (33); SDQ_34 (34); SDQ_35 (35); SDQ 36 (36):

SDQ_37 (37); SDQ_38 (38); SDQ_39 (39); SDQ 40 (40);

SDQ 41 (41); SDQ 42 (42); SDQ 43 (43); SDQ 44 (44);

SDQ 45 (45); SDQ 46 (46); SDQ 47 (47); SDQ 48 (48); 
SDQ_49 (49); SDQ_50 (50); SDQ_51 (51); SDQ_52 (52);

SDQ 53 (53); SDQ 54 (54); SDQ 55 (55); SDQ 56 (56);

SDQ_57 (57); SDQ_58 (58); SDQ_59 (59); SDQ_60 (60);

SDQ 61 (61); SDQ 62 (62); SDQ 63 (63); SDQ 64 (64);

SDQ_65 (65); SDQ_66 (66); SDQ_67 (67); SDQ_68 (68);

SDQ 69 (69); SDQ 70 (70); SDQ 71 (71); SDQ 72 (72);

SDQ_73 (73); SDQ_74 (74); SDQ_75 (75); SDQ_76 (76); 
Title: Multiple Group Variance-Covariance (Loadings, Intercepts, Uniquenesses, CUs, Variances, Covariances) Invariance Model of the SDQ-I (Real Data)

\section{! [...]Model section only}

GLOBAL BY SDQ 1 SDQ 2 SDQ 3 SDQ 4 SDQ 5 SDQ 6 SDQ 7 SDQ 8 SDQ 9 SDQ 10

SDQ_11 SDQ_12 SDQ_13 SDQ_14 SDQ_15 SDQ_16 SDQ_17 SDQ_18 SDQ_19 SDQ_20

$\mathrm{SDQ} 21 \mathrm{SDQ} 22 \mathrm{SDQ} 23 \mathrm{SDQ} 24 \mathrm{SDQ} 25 \mathrm{SDQ} 26 \mathrm{SDQ} 27 \mathrm{SDQ} 28 \mathrm{SDQ} 29 \mathrm{SDQ} 30$

SDQ_31 SDQ_32 SDQ_33 SDQ_34 SDQ_35 SDQ_36 SDQ_37 SDQ_38 SDQ_39 SDQ_40

$\mathrm{SDQ} 41 \mathrm{SDQ} 42 \mathrm{SDQ} 43 \mathrm{SDQ} 44 \mathrm{SDQ} 45 \mathrm{SDQ} 46 \mathrm{SDQ} 47 \mathrm{SDQ} 48 \mathrm{SDQ} 49 \mathrm{SDQ} 50$

SDQ_51 SDQ_52 SDQ_53 SDQ_54 SDQ_55 SDQ_56 SDQ_57 SDQ_58 SDQ_59 SDQ_60

SDQ_61 SDQ_62 SDQ_63 SDQ_64 SDQ_65 SDQ_66 SDQ_67 SDQ_68 SDQ_69 SDQ_70

SDQ_71 SDQ 72 SDQ 73 SDQ 74 SDQ_75 SDQ 76 (*t1);

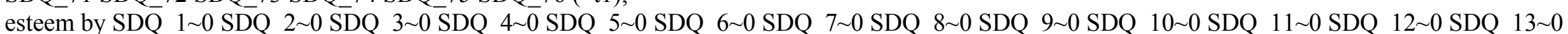
SDQ_14 0 SDQ_15 0 SDQ_16 0 SDQ_17 0 SDQ_18 0 SDQ_19 0 SDQ_20 0 SDQ_21 0 SDQ_22 0 SDQ_23 0 SDQ_24 0 SDQ 25 0 SDQ_26 0 SDQ 27 0 SDQ 28 0 SDQ 29 SDQ 30 0 SDQ 31 0 SDQ 32 0 SDQ 33 0 SDQ 34 0 SDQ 35 0 SDQ 36 0 SDQ 37 SDQ 38 0 SDQ 39 0 SDQ 40 0 SDQ_41 0 SDQ 42 0 SDQ_43 0 SDQ 44 0 SDQ 45 SDQ_46 0 SDQ_47 0 SDQ_48 0 SDQ_49 0 SDQ_50 0 SDQ_51 0 SDQ 52 0 SDQ_53 SDQ 54 $\sim 0$ SDQ $55 \sim 0$ SDQ_56 $\sim$ SDQ $57 \sim 0$ SDQ_58 0 SDQ 59 0 SDQ_60 0 SDQ 61 SDQ_62 0 SDQ_63 0 SDQ_64 0 SDQ_65 0 SDQ 66 0 SDQ 67 SDQ 68 0 SDQ 69 0 SDQ 70 SDQ 71 0 SDQ 72 SDQ 73 0 SDQ 74 SDQ 75 0 SDQ 76 (*t1);

peer by SDQ_1 0 SDQ_2 0 SDQ_3 0 SDQ_4 0 SDQ_5 0 SDQ_6 0 SDQ_7 SDQ_8 0 SDQ_9 0 SDQ_10 0 SDQ_11 0 SDQ_12 0 SDQ_13 0 SDQ 14 SDQ $15 \sim 0$ SDQ $16 \sim 0$ SDQ 17 0 SDQ 18 0 SDQ_19 0 SDQ 20 0 SDQ 21 SDQ 22 0 SDQ 23 0 SDQ_24 0 SDQ 25 0 SDQ 26 0 SDQ_27 0 SDQ 28 SDQ_29 0 SDQ_30 0 SDQ_31 0 SDQ_32 0 SDQ_33 0 SDQ_34 0 SDQ_35 0 SDQ_36 SDQ_37 0 SDQ_38 0 SDQ_39 0 SDQ _40 0 SDQ 41 0 SDQ 42 $\sim$ SDQ 43 0 SDQ 44 SDQ 45 0 SDQ 46 0 SDQ 47 0 SDQ 48 0 SDQ 49 0 SDQ_50 0 SDQ 51 0 SDQ 52 SDQ_53 0 SDQ_54 0 SDQ_55 0 SDQ_56 0 SDQ_57 0 SDQ 58 0 SDQ_59 0 SDQ_60 SDQ_61 0 SDQ_62 0 SDQ_63 0 SDQ_64 0 SDQ_65 0 SDQ 66 0 SDQ 67 0 SDQ 68 0 SDQ 69 SDQ 70 0 SDQ 71 0 SDQ 72 0 SDQ 73 0 SDQ 74 0 SDQ $75 \sim 0$ SDQ 76 0 $(* 11)$; appear by SDQ_1 SDQ_2 0 SDQ_3 0 SDQ_4 0 SDQ_5 0 SDQ_6 0 SDQ_7 0 SDQ 8 SDQ_9 0 SDQ_10 0 SDQ_11 0 SDQ_12 0 SDQ_13 0 SDQ 14 0 SDQ 15 SDQ 16 0 SDQ 17 0 SDQ 18 0 SDQ 19 0 SDQ 20 0 SDQ 21 0 SDQ 22 SDQ 23 0 SDQ 24 0 SDQ 25 0 SDQ 26 0 SDQ_27 0 SDQ_28 0 SDQ_29 0 SDQ_30 SDQ_31 0 SDQ_32 0 SDQ_33 0 SDQ_34 0 SDQ_35 0 SDQ_36 0 SDQ_37 0 SDQ_38 SDQ_39 0

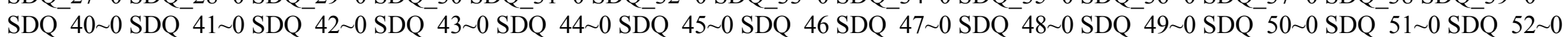
SDQ_53 0 SDQ_54 SDQ 55 0 SDQ 56 $\sim 0$ SDQ 57 $\sim 0$ SDQ 58 $\sim 0$ SDQ 59 $\sim 0$ SDQ $60 \sim 0$ SDQ_61 0 SDQ_62 SDQ_63 0 SDQ_64 0 SDQ_65 0 SDQ $66 \sim 0$ SDQ $67 \sim 0$ SDQ $68 \sim 0$ SDQ $69 \sim 0$ SDQ $70 \sim 0$ SDQ $71 \sim 0$ SDQ $72 \sim 0$ SDQ $73 \sim 0$ SDQ $74 \sim 0$ SDQ $75 \sim 0$ SDQ $76 \sim 0(* t 1)$;

phy by SDQ 1 0 SDQ_2 0 SDQ 3 SDQ 4 0 SDQ 5 0 SDQ_6 6 SDQ 7 7 0 SDQ_8 0 SDQ_9 0 SDQ_10 SDQ_11 0 SDQ_12 0 SDQ_13 0 SDQ_14 0 SDQ 15 0 SDQ 16 0 SDQ 17 SDQ 18 0 SDQ 19 0 SDQ 20 0 SDQ 21 0 SDQ 22 0 SDQ 23 0 SDQ 24 SDQ 25 0 SDQ 26 0 SDQ 27 0 SDQ_28 0 SDQ_29 0 SDQ_30 0 SDQ_31 0 SDQ_32 SDQ_33 0 SDQ_34 0 SDQ_35 0 SDQ_36 0 SDQ_37 0 SDQ_38 0 SDQ_39 0 SDQ_40 SDQ 41 0 SDQ 42 0 SDQ 43 0 SDQ 44 0 SDQ 45 0 SDQ 46 0 SDQ 47 0 SDQ 48 SDQ 49 0 SDQ 50 0 SDQ 51 0 SDQ 52 0 SDQ 53 0 SDQ_54 0 SDQ_55 0 SDQ_56 SDQ_57 0 SDQ_58 0 SDQ_59 0 SDQ_60 0 SDQ_61 0 SDQ_62 0 SDQ_63 0 SDQ_64 SDQ_65 0 SDQ_66 0 SDQ_67 0 SDQ_68 0 SDQ_69 0 SDQ_70 0 SDQ_71 0 SDQ_72 0 SDQ_73 0 SDQ_74 0 SDQ_75 0 SDQ_76 0 $(* \operatorname{t} 1)$; 
parent by SDQ 1 1 0 SDQ 2 0 SDQ _3 0 SDQ 4 0 SDQ 5 SDQ 6 0 SDQ 7 7 0 SDQ 8 0 SDQ 9 0 SDQ 10 0 SDQ 11 0 SDQ 12 SDQ 13 0 SDQ_14 0 SDQ_15 0 SDQ_16 0 SDQ_17 0 SDQ 18 0 SDQ_19 SDQ 20 0 SDQ_21 0 SDQ_22 0 SDQ_23 0 SDQ_24 0 SDQ_25 0 SDQ_26 SDQ_27 0 SDQ_28 0 SDQ_29 0 SDQ_30 0 SDQ_31 0 SDQ_32 0 SDQ_33 0 SDQ_34 SDQ_35 0 SDQ_36 0 SDQ_37 0 SDQ_38 0 SDQ_39 0 SDQ 40 0 SDQ 41 0 SDQ 42 SDQ 43 0 SDQ 44 0 SDQ 45 0 SDQ 46 0 SDQ 47 0 SDQ 48 0 SDQ 49 0 SDQ 50 SDQ 51 0 SDQ 52 0 SDQ_53 0 SDQ_54 0 SDQ_55 0 SDQ_56 0 SDQ_57 0 SDQ_58 SDQ_59 0 SDQ_60 0 SDQ_61 0 SDQ_62 0 SDQ_63 0 SDQ_64 0 SDQ_65 0 SDQ 66 SDQ 67 0 SDQ 68 0 SDQ 69 0 SDQ 70 0 SDQ 71 0 SDQ 72 0 SDQ 73 0 SDQ 74 0 SDQ 75 0 SDQ 76 0 (*t1);

schocom by SDQ_1 0 SDQ_2 SDQ_3 0 SDQ 4 0 SDQ_5 0 SDQ_6 0 SDQ_7 0 SDQ_8 0 SDQ_9 0 SDQ_10 0 SDQ_11 0 SDQ_12 0 SDQ_13 0 SDQ 14 0 SDQ 15 0 SDQ 16 SDQ 17 0 SDQ 18 0 SDQ 19 0 SDQ 20 0 SDQ 21 0 SDQ 22 0 SDQ 23 0 SDQ 24 0 SDQ 25 0 SDQ 26 0 SDQ_27 0 SDQ_28 0 SDQ_29 0 SDQ_30 0 SDQ_31 SDQ_32 0 SDQ_33 0 SDQ_34 0 SDQ_35 0 SDQ_36 0 SDQ_37 0 SDQ_38 0 SDQ_39 0 SDQ 40 0 SDQ 41 0 SDQ 42 0 SDQ 43 0 SDQ 44 0 SDQ 45 0 SDQ 46 0 SDQ 47 SDQ 48 0 SDQ 49 0 SDQ 50 0 SDQ 51 0 SDQ 52 0 SDQ_53 0 SDQ_54 0 SDQ_55 0 SDQ_56 0 SDQ_57 0 SDQ_58 0 SDQ_59 0 SDQ_60 0 SDQ_61 0 SDQ_62 0 SDQ_63 SDQ_64 0 SDQ_65 0 SDQ 66 0 SDQ 67 0 SDQ 68 0 SDQ 69 0 SDQ 70 0 SDQ 71 0 SDQ 72 0 SDQ 73 0 SDQ 74 0 SDQ $75 \sim 0$ SDQ 76 0 (*t1);

schoaff by SDQ 1 1 0 SDQ_2 0 SDQ _3 0 SDQ 4 0 SDQ 5 0 SDQ 6 0 SDQ 7 0 SDQ 8 0 SDQ 9 SDQ_10 0 SDQ_11 0 SDQ_12 0 SDQ_13 0 SDQ_14 0 SDQ_15 0 SDQ_16 0 SDQ_17 0 SDQ_18 0 SDQ_19 0 SDQ_20 0 SDQ_21 0 SDQ_22 0 SDQ_23 SDQ_24 0 SDQ_25 0 SDQ_26 0 SDQ_27 0 SDQ_28 0 SDQ_29 0 SDQ_30 0 SDQ_31 0 SDQ_32 0 SDQ_33 0 SDQ_34 0 SDQ_35 0 SDQ_36 0 SDQ_37 0 SDQ_38 0 SDQ_39 SDQ 40 0 SDQ 41 0 SDQ_42 0 SDQ_43 0 SDQ 44 0 SDQ_45 0 SDQ 46 0 SDQ 47 0 SDQ_48 0 SDQ 49 0 SDQ_50 0 SDQ_51 0 SDQ_52 0 SDQ 53 0 SDQ 54 $\sim 0$ SDQ 55 SDQ 56 $\sim 0$ SDQ 57 $\sim 0$ SDQ 58 $\sim 0$ SDQ_59 0 SDQ 60 0 SDQ_61 0 SDQ_62 0 SDQ_63 0 SDQ_64 0 SDQ_65 0 SDQ 66 0 SDQ 67 0 SDQ 68 0 SDQ 69 0 SDQ 70 0 SDQ 71 SDQ 72 0 SDQ 73 0 SDQ 74 0 SDQ $75 \sim 0$ SDQ 76 0 (*t1);

Germcom by SDQ 1 0 SDQ 2 0 SDQ 3 0 SDQ 4 SDQ 5 0 SDQ 6 0 SDQ 7 0 SDQ 8 0 SDQ 9 0 SDQ 10 0 SDQ 11 0 SDQ 12 0 SDQ 13 0 SDQ_14 0 SDQ_15 0 SDQ_16 0 SDQ_17 0 SDQ_18 SDQ_19 0 SDQ_20 0 SDQ_21 0 SDQ_22 0 SDQ_23 0 SDQ_24 0 SDQ_25 0 SDQ_26 0 SDQ 27 0 SDQ 28 0 SDQ 29 0 SDQ 30 0 SDQ 31 0 SDQ 32 0 SDQ 33 SDQ 34 0 SDQ 35 0 SDQ 36 0 SDQ 37 0 SDQ 38 0 SDQ 39 0 SDQ_40 0 SDQ_41 0 SDQ_42 0 SDQ_43 0 SDQ_44 0 SDQ_45 0 SDQ_46 0 SDQ_47 0 SDQ_48 0 SDQ_49 SDQ_50 0 SDQ_51 0 SDQ_52 0 SDQ 53 0 SDQ 54 0 SDQ 55 0 SDQ 56 0 SDQ 57 0 SDQ 58 0 SDQ 59 0 SDQ 60 0 SDQ 61 0 SDQ 62 0 SDQ 63 0 SDQ 64 0 SDQ 65 0 SDQ_66 0 SDQ_67 0 SDQ_68 0 SDQ_69 0 SDQ_70 0 SDQ_71 0 SDQ 72 0 SDQ_73 SDQ 74 $\sim 0$ SDQ_75 0 SDQ 76 $\sim 0(* 1)$;

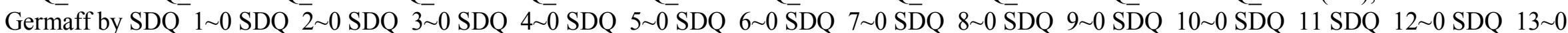
SDQ_14 0 SDQ_15 0 SDQ_16 0 SDQ_17 0 SDQ_18 0 SDQ_19 0 SDQ_20 0 SDQ_21 0 SDQ_22 0 SDQ_23 0 SDQ_24 0 SDQ_25 SDQ_26 0 SDQ 27 0 SDQ 28 0 SDQ 29 0 SDQ 30 0 SDQ 31 0 SDQ 32 0 SDQ 33 0 SDQ 34 0 SDQ 35 0 SDQ 36 0 SDQ 37 0 SDQ 38 0 SDQ 39 0

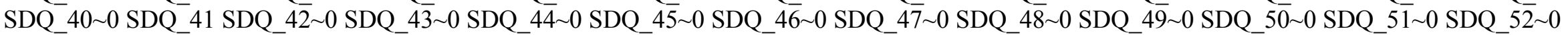
SDQ 53 0 SDQ 54 0 SDQ 55 0 SDQ 56 0 SDQ 57 SDQ 58 0 SDQ 59 0 SDQ 60 0 SDQ 61 0 SDQ $62 \sim 0$ SDQ $63 \sim 0$ SDQ $64 \sim 0$ SDQ 65 SDQ_66 0 SDQ 67 0 SDQ_68 0 SDQ_69 0 SDQ_70 0 SDQ_71 0 SDQ_72 0 SDQ_73 0 SDQ_74 0 SDQ 75 0 SDQ_76 0 $(* 11)$;

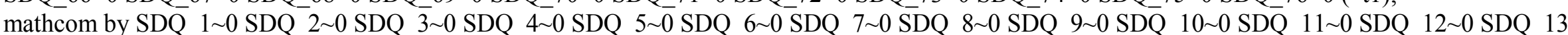
SDQ 14 0 SDQ 15 0 SDQ_16 0 SDQ 17 0 SDQ 18 0 SDQ_19 0 SDQ 20 0 SDQ 21 0 SDQ 22 0 SDQ 23 0 SDQ 24 0 SDQ 25 0 SDQ 26 0 SDQ 27 SDQ 28 0 SDQ 29 0 SDQ 30 0 SDQ 31 0 SDQ 32 0 SDQ 33 0 SDQ 34 0 SDQ 35 0 SDQ 36 0 SDQ 37 0 SDQ 38 0 SDQ 39 0 SDQ 40 0 SDQ 41 0 SDQ 42 0 SDQ 43 SDQ 44 0 SDQ 45 0 SDQ 46 0 SDQ 47 0 SDQ 48 0 SDQ 49 0 SDQ 50 0 SDQ 51 0 SDQ 52 0 SDQ_53 0 SDQ_54 0 SDQ_55 0 SDQ_56 0 SDQ_57 0 SDQ_58 0 SDQ_59 SDQ_60 0 SDQ_61 0 SDQ_62 0 SDQ_63 0 SDQ_64 0 SDQ_65 0 
SDQ 66 0 SDQ 67 0 SDQ 68 0 SDQ 69 0 SDQ 70 0 SDQ 71 0 SDQ 72 0 SDQ 73 0 SDQ 74 0 SDQ 75 SDQ 76 0 $(* 11)$;

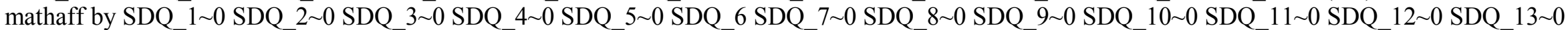
SDQ_14 0 SDQ_15 0 SDQ_16 0 SDQ_17 0 SDQ_18 0 SDQ_19 0 SDQ_20 SDQ_21 0 SDQ_22 0 SDQ_23 0 SDQ_24 0 SDQ_25 0 SDQ_26 0 SDQ 27 0 SDQ 28 0 SDQ 29 0 SDQ 30 0 SDQ 31 0 SDQ 32 0 SDQ 33 0 SDQ 34 0 SDQ 35 SDQ 36 0 SDQ 37 0 SDQ 38 0 SDQ 39 0 SDQ_40 0 SDQ_41 0 SDQ_42 0 SDQ_43 0 SDQ_44 0 SDQ_45 0 SDQ_46 0 SDQ_47 0 SDQ_48 0 SDQ_49 0 SDQ_50 0 SDQ_51 SDQ_52 0 SDQ 53 0 SDQ 54 0 SDQ 55 0 SDQ 56 0 SDQ 57 0 SDQ 58 0 SDQ 59 0 SDQ 60 0 SDQ 61 0 SDQ 62 0 SDQ 63 0 SDQ 64 0 SDQ 65 0 SDQ_66 0 SDQ_67 0 SDQ_68 SDQ_69 0 SDQ_70 0 SDQ_71 0 SDQ_72 0 SDQ_73 0 SDQ_74 0 SDQ_75 0 SDQ_76 0 $(* 1)$;

! Method Factor (negative items)

MF BY SDQ 30* (MF1)

SDQ 17 SDQ 12 SDQ 21 SDQ 47 SDQ 23 SDQ 33 SDQ 65 SDQ 75 SDQ 6 SDQ 37 SDQ 61 (MF2-MF12);

MF@1; [MF@0];

MFWITH GLOBAL@0 esteem@0 peer@0 appear@0 phy@0 parent@0 schocom@0

schoaff@0.Germcom@0 Germaff@0 MathAff@0 MathCom@0;

! correlated uniquenesses between parallel worded items

SDQ_11 with SDQ_51 (cu1); SDQ_11 with SDQ 71 (cu2); SDQ_51 with SDQ 71 (cu3);

SDQ 25 with SDQ 35 (cu4); SDQ 25 with SDQ 39 (cu5); SDQ 35 with SDQ 39 (cu6);

SDQ_41 with SDQ_68 (cu7); SDQ_41 with SDQ_9 (cu8); SDQ_68 with SDQ_9 (cu9);

SDQ_57 with SDQ_20 (cu10); SDQ 57 with SDQ_55 (cu11); SDQ 20 with SDQ 55 (cu12);

SDQ 23 with SDQ 6 (cu13); SDQ 23 with SDQ 65 (cu14); SDQ 6 with SDQ 65 (cu15);

SDQ 4 with SDQ 27 (cu16); SDQ 4 with SDQ 16 (cu17); SDQ 27 with SDQ 16 (cu18);

SDQ 18 with SDQ 59 (cu19); SDQ 18 with SDQ 2 (cu20); SDQ 59 with SDQ 2 (cu21);

SDQ_49 with SDQ_13 (cu22); SDQ_49 with SDQ_63 (cu23); SDQ_13 with SDQ_63 (cu24);

SDQ 73 with SDQ 43 (cu25); SDQ 73 with SDQ 31 (cu26); SDQ 43 with SDQ 31 (cu27);

SDQ_47 with SDQ_75 (cu28); SDQ_47 with SDQ_33 (cu29); SDQ_75 with SDQ_33 (cu30);

SDQ 1 (1); SDQ 2 (2); SDQ 3 (3); SDQ 4 (4); SDQ 5 (5); SDQ 6 (6); SDQ 7 (7); SDQ 8 (8);

SDQ_9 (9); SDQ_10 (10); SDQ_11 (11); SDQ_12 (12);

SDQ 13 (13); SDQ 14 (14); SDQ 15 (15); SDQ 16 (16);

SDQ_17 (17); SDQ_18 (18); SDQ_19 (19); SDQ_20 (20);

SDQ 21 (21); SDQ 22 (22); SDQ 23 (23); SDQ 24 (24);

SDQ_25 (25); SDQ_26 (26); SDQ_27 (27); SDQ_28 (28);

SDQ 29 (29); SDQ 30 (30); SDQ 31 (31); SDQ 32 (32);

SDQ 33 (33); SDQ_34 (34); SDQ 35 (35); SDQ_36 (36):

SDQ 37 (37); SDQ 38 (38); SDQ 39 (39); SDQ 40 (40);

SDQ 41 (41); SDQ 42 (42); SDQ 43 (43); SDQ 44 (44);

SDQ 45 (45); SDQ 46 (46); SDQ 47 (47); SDQ 48 (48); 
SDQ 49 (49); SDQ 50 (50); SDQ 51 (51); SDQ 52 (52);

SDQ 53 (53); SDQ 54 (54); SDQ 55 (55); SDQ 56 (56);

SDQ_57 (57); SDQ_58 (58); SDQ_59 (59); SDQ_60 (60);

SDQ 61 (61); SDQ 62 (62); SDQ 63 (63); SDQ 64 (64);

SDQ_65 (65); SDQ_66 (66); SDQ_67 (67); SDQ_68 (68);

SDQ 69 (69); SDQ 70 (70); SDQ 71 (71); SDQ 72 (72);

SDQ_73 (73); SDQ_74 (74); SDQ_75 (75); SDQ_76 (76);

! invariance of variances and covariances

global-mathaff@1;

esteem with peer (a31); esteem with phys (a32); esteem with parent (a33);

esteem with schocom (a34); esteem with schoaff (a35); esteem with Germcom (a36);

esteem with Germaff (a37); esteem with mathcom (a38); esteem with mathaff (a39);

esteem with appear (a40); esteem with global (a30);

peer with phys (a41); peer with parent (a42); peer with schocom (a43);

peer with schoaff (a44); peer with Germcom (a45); peer with Germaff (a46);

peer with mathcom (a47); peer with mathaff (a48); peer with appear (a49);

peer with global (a50);

phys with parent (a51); phys with schocom (a52); phys with schoaff (a53);

phys with Germcom (a54); phys with Germaff (a55); phys with mathcom (a56);

phys with mathaff (a57); phys with appear (a58); phys with global (a59);

parent with schocom (a60); parent with schoaff (a61); parent with Germcom (a62);

parent with Germaff (a63); parent with mathcom (a64);

parent with mathaff (a65); parent with appear (a66); parent with global (a67);

schocom with schoaff (a70); schocom with Germcom (a71); schocom with Germaff (a72);

schocom with mathcom (a73); schocom with mathaff (a74); schocom with appear (a75);

schocom with global (a76);

schoaff with Germcom (a80); schoaff with Germaff (a81); schoaff with mathcom (a82);

schoaff with mathaff (a83); schoaff with appear (a84); schoaff with global (a85);

Germcom with Germaff (a90); Germcom with mathcom (a91); Germcom with mathaff (a92);

Germcom with appear (a93); Germcom with global (a94); Germaff with mathcom (a100);

Germaff with mathaff (a101); Germaff with appear (a102); Germaff with global (a103);

mathcom with mathaff (a110); mathcom with appear (a111); mathcom with global (a112);

mathaff with appear (a113); mathaff with global (a114);

appear with global (a115);

model female: 
! Method Factor (negative items)

MF BY SDQ 30* (MF1)

SDQ_17 SDQ 12 SDQ_21 SDQ 47 SDQ 23 SDQ 33 SDQ 65 SDQ 75 SDQ 6 SDQ_37 SDQ_61 (MF2-MF12);

MF@1; [MF*];

MFWITH GLOBAL@0 esteem@0 peer@0 appear@0 phy@0 parent@0 schocom@0

schoaff@0 Germcom@0 Germaff@0 MathAff@0 MathCom@0;

! correlated uniquenesses between parallel worded items

SDQ 11 with SDQ 51 (cu1); SDQ 11 with SDQ 71 (cu2); SDQ 51 with SDQ 71 (cu3);

SDQ_25 with SDQ_35 (cu4); SDQ_25 with SDQ_39 (cu5); SDQ_35 with SDQ_39 (cu6);

SDQ 41 with SDQ 68 (cu7); SDQ 41 with SDQ 9 (cu8); SDQ 68 with SDQ 9 (cu9);

SDQ 57 with SDQ_20 (cu10); SDQ 57 with SDQ_55 (cu11); SDQ_20 with SDQ 55 (cu12);

SDQ 23 with SDQ 6 (cu13); SDQ 23 with SDQ_65 (cu14); SDQ 6 with SDQ 65 (cu15);

SDQ 4 with SDQ 27 (cu16); SDQ 4 with SDQ_16 (cu17); SDQ_27 with SDQ_16 (cu18);

SDQ 18 with SDQ 59 (cu19); SDQ 18 with SDQ 2 (cu20); SDQ 59 with SDQ 2 (cu21);

SDQ_49 with SDQ_13 (cu22); SDQ_49 with SDQ_63 (cu23); SDQ_13 with SDQ_63 (cu24);

SDQ_73 with SDQ 43 (cu25); SDQ 73 with SDQ_31 (cu26); SDQ 43 with SDQ 31 (cu27);

SDQ 47 with SDQ 75 (cu28); SDQ 47 with SDQ 33 (cu29); SDQ 75 with SDQ 33 (cu30);

SDQ_1 (1); SDQ_2 (2); SDQ_3 (3); SDQ_4 (4); SDQ_5 (5); SDQ_6 (6); SDQ_7 (7); SDQ_8 (8);

SDQ 9 (9); SDQ 10 (10); SDQ 11 (11); SDQ 12 (12);

SDQ_13 (13); SDQ_14 (14); SDQ_15 (15); SDQ_16 (16);

SDQ 17 (17); SDQ 18 (18); SDQ 19 (19); SDQ 20 (20);

SDQ_21 (21); SDQ_22 (22); SDQ_23 (23); SDQ_24 (24);

SDQ 25 (25); SDQ 26 (26); SDQ 27 (27); SDQ 28 (28);

SDQ_29 (29); SDQ_30 (30); SDQ_31 (31); SDQ_32 (32);

SDQ 33 (33); SDQ 34 (34); SDQ 35 (35); SDQ 36 (36):

SDQ_37 (37); SDQ_38 (38); SDQ_39 (39); SDQ_40 (40);

SDQ 41 (41); SDQ 42 (42); SDQ 43 (43); SDQ 44 (44);

SDQ_45 (45); SDQ_46 (46); SDQ_47 (47); SDQ_48 (48);

SDQ 49 (49); SDQ 50 (50); SDQ 51 (51); SDQ 52 (52);

SDQ_53 (53); SDQ_54 (54); SDQ_55 (55); SDQ_56 (56);

SDQ 57 (57); SDQ_58 (58); SDQ_59 (59); SDQ 60 (60);

SDQ_61 (61); SDQ_62 (62); SDQ_63 (63); SDQ 64 (64);

SDQ_65 (65); SDQ_66 (66); SDQ_67 (67); SDQ_68 (68);

SDQ 69 (69); SDQ 70 (70); SDQ 71 (71); SDQ 72 (72);

SDQ_73 (73); SDQ_74 (74); SDQ_75 (75); SDQ_76 (76); 
! invariance of variances and covariances

global-mathaff@1;

esteem with peer (a31); esteem with phys (a32); esteem with parent (a33);

esteem with schocom (a34); esteem with schoaff (a35); esteem with Germcom (a36);

esteem with Germaff (a37); esteem with mathcom (a38); esteem with mathaff (a39);

esteem with appear (a40); esteem with global (a30);

peer with phys (a41); peer with parent (a42); peer with schocom (a43);

peer with schoaff (a44); peer with Germcom (a45); peer with Germaff (a46);

peer with mathcom (a47); peer with mathaff (a48); peer with appear (a49);

peer with global (a50);

phys with parent (a51); phys with schocom (a52); phys with schoaff (a53);

phys with Germcom (a54); phys with Germaff (a55); phys with mathcom (a56);

phys with mathaff (a57); phys with appear (a58); phys with global (a59);

parent with schocom (a60); parent with schoaff (a61); parent with Germcom (a62);

parent with Germaff (a63); parent with mathcom (a64);

parent with mathaff (a65); parent with appear (a66); parent with global (a67);

schocom with schoaff (a70); schocom with Germcom (a71); schocom with Germaff (a72);

schocom with mathcom (a73); schocom with mathaff (a74); schocom with appear (a75);

schocom with global (a76);

schoaff with Germcom (a80); schoaff with Germaff (a81); schoaff with mathcom (a82);

schoaff with mathaff (a83); schoaff with appear (a84); schoaff with global (a85);

Germcom with Germaff (a90); Germcom with mathcom (a91); Germcom with mathaff (a92);

Germcom with appear (a93); Germcom with global (a94); Germaff with mathcom (a100);

Germaff with mathaff (a101); Germaff with appear (a102); Germaff with global (a103);

mathcom with mathaff (a110); mathcom with appear (a111); mathcom with global (a112);

mathaff with appear (a113); mathaff with global (a114);

appear with global (a115); 
Title: Multiple Group Latent Means (Loadings, Intercepts, Uniquenesses, CUs, Variances, Covariances, Latent Means) Invariance Model of the SDQ-I (Real Data)

\section{! [...]Model section only}

! [...]Model section only

GLOBAL BY SDQ_1 SDQ_2 SDQ_3 SDQ 4 SDQ_5 SDQ_6 SDQ_7 SDQ_8 SDQ_9 SDQ_10

SDQ 11 SDQ 12 SDQ 13 SDQ 14 SDQ 15 SDQ 16 SDQ 17 SDQ 18 SDQ 19 SDQ 20

SDQ_21 SDQ_22 SDQ_23 SDQ_24 SDQ_25 SDQ_26 SDQ_27 SDQ_28 SDQ_29 SDQ_30

SDQ $31 \mathrm{SDQ} 32 \mathrm{SDQ} 33 \mathrm{SDQ} 34 \mathrm{SDQ} 35 \mathrm{SDQ} 36 \mathrm{SDQ} 37 \mathrm{SDQ} 38 \mathrm{SDQ} 39 \mathrm{SDQ} 40$

SDQ_41 SDQ_42 SDQ_43 SDQ_44 SDQ_45 SDQ_46 SDQ_47 SDQ_48 SDQ_49 SDQ_50

SDQ 51 SDQ 52 SDQ 53 SDQ 54 SDQ 55 SDQ 56 SDQ 57 SDQ 58 SDQ 59 SDQ 60

SDQ_61 SDQ_62 SDQ_63 SDQ_64 SDQ_65 SDQ_66 SDQ_67 SDQ_68 SDQ_69 SDQ_70

SDQ 71 SDQ 72 SDQ 73 SDQ 74 SDQ 75 SDQ $76(* t 1)$

esteem by SDQ 1 0 SDQ 2 0 SDQ 3 0 SDQ 4 0 SDQ_5 0 SDQ_6 0 SDQ 7 0 SDQ_8 0 SDQ_9 0 SDQ_10 0 SDQ_11 0 SDQ_12 0 SDQ_13 0 SDQ 14 0 SDQ 15 0 SDQ 16 0 SDQ 17 0 SDQ 18 0 SDQ 19 0 SDQ 20 0 SDQ 21 0 SDQ 22 0 SDQ 23 0 SDQ 24 0 SDQ 25 0 SDQ 26 0 SDQ_27 0 SDQ_28 0 SDQ_29 SDQ_30 0 SDQ_31 0 SDQ_32 0 SDQ_33 0 SDQ_34 0 SDQ_35 0 SDQ_36 0 SDQ_37 SDQ_38 0 SDQ_39 0

SDQ _40 0 SDQ 41 0 SDQ 42 0 SDQ 43 0 SDQ 44 0 SDQ 45 SDQ 46 0 SDQ 47 0 SDQ 48 0 SDQ 49 0 SDQ 50 0 SDQ 51 0 SDQ 52 0

SDQ_53 SDQ_54 0 SDQ_55 0 SDQ_56 0 SDQ_57 0 SDQ_58 0 SDQ_59 0 SDQ_60 0 SDQ_61 SDQ_62 0 SDQ_63 0 SDQ_64 0 SDQ_65 0 SDQ $66 \sim 0$ SDQ 67 SDQ 68 0 SDQ 69 0 SDQ 70 SDQ 71 0 SDQ 72 SDQ 73 0 SDQ 74 SDQ 75 0 SDQ 76 (*t1);

peer by SDQ 1 0 SDQ 2 2 0 SDQ 3 0 SDQ 4 4 0 SDQ 5 0 SDQ 6 0 SDQ 7 SDQ 8 0 SDQ 9 0 SDQ 10 0 SDQ_11 0 SDQ_12 0 SDQ_13 0 SDQ_14 SDQ_15 0 SDQ 16 0 SDQ_17 0 SDQ_18 0 SDQ_19 0 SDQ_20 0 SDQ_21 SDQ_22 0 SDQ_23 0 SDQ_24 0 SDQ_25 0 SDQ_26 0 SDQ 27 0 SDQ 28 SDQ 29 0 SDQ 30 0 SDQ 31 0 SDQ 32 0 SDQ 33 0 SDQ 34 0 SDQ 35 0 SDQ 36 SDQ 37 0 SDQ 38 0 SDQ 39 0 SDQ_40 0 SDQ_41 0 SDQ_42 0 SDQ_43 0 SDQ_44 SDQ_45 0 SDQ_46 0 SDQ_47 0 SDQ_48 0 SDQ_49 0 SDQ_50 0 SDQ_51 0 SDQ_52 SDQ 53 0 SDQ 54 0 SDQ 55 0 SDQ 56 0 SDQ 57 0 SDQ 58 0 SDQ 59 0 SDQ 60 SDQ 61 0 SDQ 62 0 SDQ 63 0 SDQ 64 0 SDQ 65 0 SDQ_66 0 SDQ_67 0 SDQ_68 0 SDQ_69 SDQ_70 0 SDQ 71 0 SDQ_72 0 SDQ 73 $\sim 0$ SDQ_74 0 SDQ_75 0 SDQ_76 0 $(* 11)$;

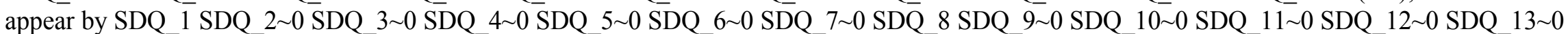
SDQ_14 0 SDQ_15 SDQ 16 0 SDQ 17 0 SDQ_18 0 SDQ_19 0 SDQ_20 0 SDQ_21 0 SDQ_22 SDQ_23 0 SDQ_24 0 SDQ_25 0 SDQ_26 0 SDQ 27 0 SDQ 28 0 SDQ 29 0 SDQ 30 SDQ 31 0 SDQ 32 0 SDQ 33 0 SDQ 34 0 SDQ 35 0 SDQ 36 0 SDQ 37 0 SDQ 38 SDQ 39 0 SDQ_40 0 SDQ_41 0 SDQ_42 0 SDQ_43 0 SDQ 44 0 SDQ 45 0 SDQ_46 SDQ_47 0 SDQ_48 0 SDQ_49 0 SDQ_50 0 SDQ_51 0 SDQ_52 0 SDQ 53 0 SDQ 54 SDQ 55 0 SDQ 56 0 SDQ 57 0 SDQ 58 0 SDQ 59 0 SDQ 60 0 SDQ 61 0 SDQ 62 SDQ $63 \sim 0$ SDQ $64 \sim 0$ SDQ $65 \sim 0$ SDQ_66 0 SDQ 67 0 SDQ_68 0 SDQ _69 0 SDQ_70 0 SDQ_71 0 SDQ_72 0 SDQ 73 0 SDQ_74 0 SDQ_75 0 SDQ_76 0 (*t1);

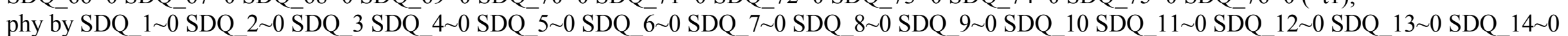
SDQ 15 0 SDQ_16 0 SDQ_17 SDQ 18 0 SDQ 19 0 SDQ_20 0 SDQ 21 0 SDQ 22 0 SDQ_23 0 SDQ 24 SDQ 25 0 SDQ 26 0 SDQ 27 0 SDQ 28 0 SDQ 29 0 SDQ 30 0 SDQ 31 0 SDQ $32 \mathrm{SDQ}$ 33 0 SDQ 34 0 SDQ 35 0 SDQ 36 0 SDQ 37 0 SDQ 38 0 SDQ 39 0 SDQ 40 SDQ 41 0 SDQ 42 0 SDQ 43 0 SDQ 44 0 SDQ 45 0 SDQ 46 0 SDQ 47 0 SDQ 48 SDQ 49 0 SDQ 50 0 SDQ 51 0 SDQ 52 0 SDQ 53 0 SDQ_54 0 SDQ_55 0 SDQ_56 SDQ_57 0 SDQ_58 0 SDQ_59 0 SDQ_60 0 SDQ_61 0 SDQ_62 0 SDQ_63 0 SDQ_64 SDQ_65 0 SDQ_66 0 
SDQ _67 0 SDQ 68 0 SDQ 69 0 SDQ 70 0 SDQ 71 0 SDQ 72 0 SDQ 73 0 SDQ 74 0 SDQ 75 0 SDQ 76 0 (*t1); parent by SDQ 1 0 SDQ 2 0 SDQ 3 0 SDQ 4 0 SDQ 5 SDQ 6 0 SDQ 7 0 SDQ 8 0 SDQ 9 0 SDQ 10 0 SDQ 11 0 SDQ 12 SDQ 13 0 SDQ_14 0 SDQ_15 0 SDQ_16 0 SDQ_17 0 SDQ_18 0 SDQ_19 SDQ_20 0 SDQ_21 0 SDQ_22 0 SDQ_23 0 SDQ_24 0 SDQ_25 0 SDQ_26 SDQ 27 0 SDQ 28 0 SDQ 29 0 SDQ 30 0 SDQ 31 0 SDQ 32 0 SDQ 33 0 SDQ 34 SDQ 35 0 SDQ 36 0 SDQ 37 0 SDQ 38 0 SDQ 39 0 SDQ_40 0 SDQ_41 0 SDQ_42 SDQ_43 0 SDQ_44 0 SDQ_45 0 SDQ_46 0 SDQ_47 0 SDQ_48 0 SDQ_49 0 SDQ_50 SDQ_51 0 SDQ_52 0 SDQ 53 0 SDQ 54 0 SDQ 55 0 SDQ 56 0 SDQ 57 0 SDQ 58 SDQ 59 0 SDQ 60 0 SDQ 61 0 SDQ 62 0 SDQ 63 0 SDQ 64 0 SDQ 65 0 SDQ_66 SDQ_67 0 SDQ_68 0 SDQ_69 0 SDQ_70 0 SDQ 71 $\sim 0$ SDQ_72 0 SDQ_73 0 SDQ_74 0 SDQ_75 0 SDQ 76 0 $(* 11)$;

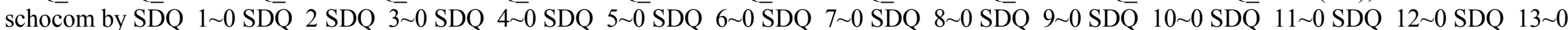
SDQ 14 0 SDQ 15 0 SDQ_16 SDQ 17 0 SDQ_18 0 SDQ 19 0 SDQ 20 0 SDQ 21 $\sim 0$ SDQ 22 0 SDQ 23 0 SDQ 24 0 SDQ 25 0 SDQ 26 0 SDQ 27 0 SDQ 28 0 SDQ 29 0 SDQ 30 0 SDQ 31 SDQ 32 0 SDQ 33 0 SDQ 34 0 SDQ 35 0 SDQ 36 0 SDQ 37 0 SDQ 38 0 SDQ 39 0 SDQ_40 0 SDQ_41 0 SDQ_42 0 SDQ_43 0 SDQ_44 0 SDQ 45 0 SDQ_46 0 SDQ 47 SDQ_48 0 SDQ_49 0 SDQ_50 0 SDQ_51 0 SDQ 52 0 SDQ_53 0 SDQ_54 0 SDQ_55 0 SDQ_56 0 SDQ_57 0 SDQ_58 0 SDQ_59 0 SDQ_60 0 SDQ_61 0 SDQ_62 0 SDQ_63 SDQ_64 0 SDQ_65 0 SDQ_66 0 SDQ_67 0 SDQ_68 0 SDQ 69 0 SDQ_70 0 SDQ_71 0 SDQ_72 0 SDQ_73 0 SDQ 74 0 SDQ_75 0 SDQ_76 0 (*t1);

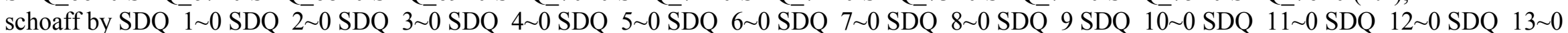
SDQ 14 0 SDQ 15 0 SDQ 16 0 SDQ 17 0 SDQ 18 0 SDQ 19 0 SDQ 20 0 SDQ 21 0 SDQ 22 0 SDQ 23 SDQ 24 0 SDQ 25 0 SDQ 26 0 SDQ_27 0 SDQ_28 0 SDQ_29 0 SDQ_30 0 SDQ_31 0 SDQ_32 0 SDQ_33 0 SDQ_34 0 SDQ_35 0 SDQ_36 0 SDQ_37 0 SDQ_38 0 SDQ_39

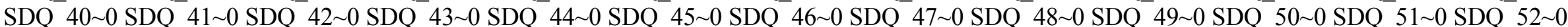
SDQ_53 0 SDQ_54 0 SDQ_55 SDQ_56 0 SDQ_57 0 SDQ_58 0 SDQ_59 0 SDQ_60 0 SDQ_61 0 SDQ_62 0 SDQ_63 0 SDQ_64 0 SDQ_65 0 SDQ 66 0 SDQ 67 0 SDQ 68 0 SDQ 69 0 SDQ 70 0 SDQ 71 SDQ 72 0 SDQ 73 0 SDQ 74 0 SDQ 75 0 SDQ 76 0 (*t1);

Germcom by SDQ 1 0 SDQ_2 0 SDQ_3 0 SDQ 4 SDQ_5 0 SDQ_6 0 SDQ_7 0 SDQ_8 0 SDQ_9 0 SDQ_10 0 SDQ_11 0 SDQ 12 0 SDQ_13 0 SDQ 14 0 SDQ 15 0 SDQ 16 0 SDQ 17 0 SDQ 18 SDQ 19 0 SDQ 20 0 SDQ 21 0 SDQ 22 0 SDQ 23 0 SDQ 24 0 SDQ 25 0 SDQ 26 0 SDQ_27 0 SDQ_28 0 SDQ_29 0 SDQ_30 0 SDQ_31 0 SDQ_32 0 SDQ_33 SDQ_34 0 SDQ_35 0 SDQ 36 0 SDQ_37 0 SDQ 38 0 SDQ 39 0 SDQ $40 \sim 0$ SDQ $41 \sim 0$ SDQ $42 \sim 0$ SDQ $43 \sim 0$ SDQ 44 0 SDQ 45 0 SDQ 46 0 SDQ $47 \sim 0$ SDQ $48 \sim 0$ SDQ 49 SDQ $50 \sim 0$ SDQ $51 \sim 0$ SDQ $52 \sim 0$ SDQ_53 0 SDQ_54 0 SDQ_55 0 SDQ_56 0 SDQ_57 0 SDQ_58 0 SDQ_59 0 SDQ_60 0 SDQ_61 0 SDQ_62 0 SDQ_63 0 SDQ_64 0 SDQ_65 0 SDQ 66 0 SDQ 67 0 SDQ 68 0 SDQ 69 0 SDQ 70 0 SDQ 71 0 SDQ 72 0 SDQ 73 SDQ 74 0 SDQ 75 0 SDQ 76 0 (*t1);

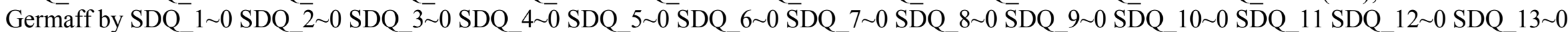
SDQ 14 0 SDQ 15 0 SDQ 16 0 SDQ 17 0 SDQ 18 0 SDQ 19 0 SDQ 20 0 SDQ 21 0 SDQ 22 0 SDQ 23 0 SDQ 24 0 SDQ 25 SDQ 26 0 SDQ 27 0 SDQ 28 0 SDQ 29 0 SDQ 30 0 SDQ 31 0 SDQ 32 0 SDQ 33 0 SDQ 34 0 SDQ 35 0 SDQ 36 0 SDQ 37 0 SDQ 38 0 SDQ 39 0 SDQ $40 \sim 0$ SDQ 41 SDQ 42 0 SDQ 43 0 SDQ 44 0 SDQ 45 $\sim 0$ SDQ $46 \sim 0$ SDQ $47 \sim 0$ SDQ $48 \sim 0$ SDQ $49 \sim 0$ SDQ $50 \sim 0$ SDQ $51 \sim 0$ SDQ $52 \sim 0$ SDQ_53 0 SDQ_54 0 SDQ 55 $~ 0$ SDQ_56 0 SDQ 57 SDQ_58 0 SDQ_59 0 SDQ_60 0 SDQ_61 0 SDQ_62 0 SDQ_63 0 SDQ_64 0 SDQ_65 SDQ $66 \sim 0$ SDQ $67 \sim 0$ SDQ $68 \sim 0$ SDQ $69 \sim 0$ SDQ $70 \sim 0$ SDQ $71 \sim 0$ SDQ $72 \sim 0$ SDQ $73 \sim 0$ SDQ $74 \sim 0$ SDQ $75 \sim 0$ SDQ $76 \sim 0$ (*t1);

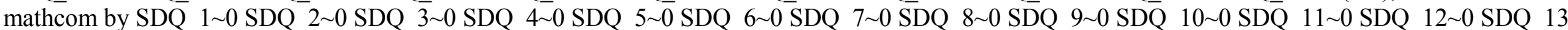

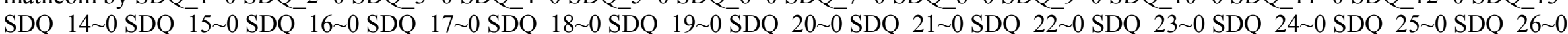
SDQ 27 SDQ 28 0 SDQ 29 0 SDQ 30 0 SDQ 31 0 SDQ 32 0 SDQ 33 0 SDQ 34 0 SDQ 35 0 SDQ 36 0 SDQ 37 0 SDQ 38 0 SDQ 39 0 SDQ 40 0 SDQ 41 0 SDQ 42 0 SDQ 43 SDQ 44 0 SDQ 45 0 SDQ 46 0 SDQ 47 0 SDQ 48 0 SDQ 49 0 SDQ 50 0 SDQ 51 0 SDQ 52 0 
SDQ_53 0 SDQ 54 $\sim$ SDQ_55 0 SDQ 56 $\sim 0$ SDQ_57 0 SDQ 58 $\sim 0$ SDQ_59 SDQ_60 0 SDQ_61 0 SDQ_62 0 SDQ_63 0 SDQ_64 0 SDQ_65 0 SDQ 66 0 SDQ 67 0 SDQ 68 0 SDQ 69 0 SDQ 70 0 SDQ 71 0 SDQ 72 0 SDQ 73 0 SDQ 74 0 SDQ 75 SDQ 76 0 $(* 11)$;

mathaff by SDQ_1 0 SDQ 2 0 SDQ_3 0 SDQ 4 4 0 SDQ_5 0 SDQ 6 SDQ 7 70 SDQ $8 \sim 0$ SDQ 9 $\sim 0$ SDQ_10 0 SDQ_11 0 SDQ_12 0 SDQ_13 0 SDQ 14 0 SDQ 15 0 SDQ 16 0 SDQ 17 0 SDQ 18 0 SDQ 19 0 SDQ 20 SDQ 21 0 SDQ 22 0 SDQ 23 0 SDQ 24 0 SDQ 25 0 SDQ 26 0 SDQ_27 0 SDQ_28 0 SDQ_29 0 SDQ_30 0 SDQ_31 0 SDQ_32 0 SDQ_33 0 SDQ_34 0 SDQ_35 SDQ_36 0 SDQ_37 0 SDQ_38 0 SDQ_39 0

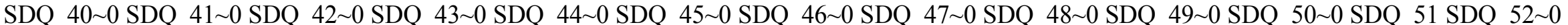
SDQ_53 0 SDQ_54 0 SDQ_55 0 SDQ_56 0 SDQ 57 0 SDQ_58 0 SDQ_59 0 SDQ_60 0 SDQ_61 0 SDQ_62 0 SDQ_63 0 SDQ_64 0 SDQ_65 0 SDQ 66 0 SDQ 67 0 SDQ 68 SDQ _69 0 SDQ_70 0 SDQ 71 0 SDQ 72 0 SDQ 73 0 SDQ 74 0 SDQ_75 0 SDQ 76 0 (*t1);

! Method Factor (negative items)

MF BY SDQ 30* (MF1)

SDQ_17 SDQ_12 SDQ_21 SDQ_47 SDQ_23 SDQ_33 SDQ_65 SDQ 75 SDQ_6 SDQ_37 SDQ_61 (MF2-MF12);

MF@1; [MF@0];

MFWITH GLOBAL@0 esteem@0 peer@0 appear@0 phy@0 parent@0 schocom@0

schoaff@0 Germcom@0 Germaff@0 MathAff@0 MathCom@0;

! correlated uniquenesses between parallel worded items

SDQ 11 with SDQ 51 (cu1); SDQ 11 with SDQ 71 (cu2); SDQ 51 with SDQ 71 (cu3);

SDQ 25 with SDQ 35 (cu4); SDQ 25 with SDQ 39 (cu5); SDQ 35 with SDQ 39 (cu6);

SDQ_41 with SDQ_68 (cu7); SDQ_41 with SDQ_9 (cu8); SDQ_68 with SDQ 9 (cu9);

SDQ 57 with SDQ_20 (cu10); SDQ 57 with SDQ 55 (cu11); SDQ 20 with SDQ 55 (cu12);

SDQ_23 with SDQ_6 (cu13); SDQ_23 with SDQ_65 (cu14); SDQ 6 with SDQ_65 (cu15);

SDQ 4 with SDQ 27 (cu16); SDQ 4 with SDQ 16 (cu17); SDQ 27 with SDQ 16 (cu18);

SDQ_18 with SDQ 59 (cu19); SDQ_18 with SDQ_2 (cu20); SDQ 59 with SDQ 2 (cu21);

SDQ 49 with SDQ 13 (cu22); SDQ 49 with SDQ 63 (cu23); SDQ 13 with SDQ 63 (cu24);

SDQ_73 with SDQ_43 (cu25); SDQ_73 with SDQ_31 (cu26); SDQ_43 with SDQ_31 (cu27);

SDQ 47 with SDQ 75 (cu28); SDQ 47 with SDQ 33 (cu29); SDQ 75 with SDQ 33 (cu30);

SDQ_1 (1); SDQ_2 (2); SDQ_3 (3); SDQ_4 (4); SDQ_5 (5); SDQ_6 (6); SDQ 7 (7); SDQ_8 (8);

SDQ 9 (9); SDQ 10 (10); SDQ 11 (11); SDQ 12 (12);

SDQ_13 (13); SDQ_14 (14); SDQ_15 (15); SDQ_16 (16);

SDQ 17 (17); SDQ 18 (18); SDQ 19 (19); SDQ 20 (20);

SDQ_21 (21); SDQ_22 (22); SDQ_23 (23); SDQ_24 (24);

SDQ 25 (25); SDQ_26 (26); SDQ_27 (27); SDQ_28 (28);

SDQ 29 (29); SDQ 30 (30); SDQ 31 (31); SDQ 32 (32);

SDQ 33 (33); SDQ 34 (34); SDQ 35 (35); SDQ 36 (36):

SDQ 37 (37); SDQ 38 (38); SDQ 39 (39); SDQ 40 (40);

SDQ_41 (41); SDQ_42 (42); SDQ_43 (43); SDQ_44 (44); 
SDQ_45 (45); SDQ_46 (46); SDQ_47 (47); SDQ 48 (48);

SDQ 49 (49); SDQ 50 (50); SDQ 51 (51); SDQ 52 (52);

SDQ_53 (53); SDQ_54 (54); SDQ_55 (55); SDQ_56 (56);

SDQ 57 (57); SDQ 58 (58); SDQ 59 (59); SDQ 60 (60);

SDQ_61 (61); SDQ_62 (62); SDQ_63 (63); SDQ_64 (64);

SDQ 65 (65); SDQ 66 (66); SDQ 67 (67); SDQ 68 (68);

SDQ_69 (69); SDQ_70 (70); SDQ_71 (71); SDQ_72 (72);

SDQ 73 (73); SDQ 74 (74); SDQ 75 (75); SDQ 76 (76);

! invariance of variances and covariances

global-mathaff@1;

esteem with peer (a31); esteem with phys (a32); esteem with parent (a33);

esteem with schocom (a34); esteem with schoaff (a35); esteem with Germcom (a36);

esteem with Germaff (a37); esteem with mathcom (a38); esteem with mathaff (a39);

esteem with appear (a40); esteem with global (a30);

peer with phys (a41); peer with parent (a42); peer with schocom (a43);

peer with schoaff (a44); peer with Germcom (a45); peer with Germaff (a46);

peer with mathcom (a47); peer with mathaff (a48); peer with appear (a49);

peer with global (a50);

phys with parent (a51); phys with schocom (a52); phys with schoaff (a53);

phys with Germcom (a54); phys with Germaff (a55); phys with mathcom (a56);

phys with mathaff (a57); phys with appear (a58); phys with global (a59);

parent with schocom (a60); parent with schoaff (a61); parent with Germcom (a62);

parent with Germaff (a63); parent with mathcom (a64);

parent with mathaff (a65); parent with appear (a66); parent with global (a67);

schocom with schoaff (a70); schocom with Germcom (a71); schocom with Germaff (a72);

schocom with mathcom (a73); schocom with mathaff (a74); schocom with appear (a75);

schocom with global (a76);

schoaff with Germcom (a80); schoaff with Germaff (a81); schoaff with mathcom (a82);

schoaff with mathaff (a83); schoaff with appear (a84); schoaff with global (a85);

Germcom with Germaff (a90); Germcom with mathcom (a91); Germcom with mathaff (a92);

Germcom with appear (a93); Germcom with global (a94); Germaff with mathcom (a100);

Germaff with mathaff (a101); Germaff with appear (a102); Germaff with global (a103);

mathcom with mathaff (a110); mathcom with appear (a111); mathcom with global (a112);

mathaff with appear (a113); mathaff with global (a114);

appear with global (a115); 
[global-mathaff@0];

model female:

! Method Factor (negative items)

MF BY SDQ 30* (MF1)

SDQ_17 SDQ_12 SDQ_21 SDQ_47 SDQ_23 SDQ_33 SDQ_65 SDQ_75 SDQ_6 SDQ_37 SDQ_61 (MF2-MF12);

MF@1; [MF@0];

MFWITH GLOBAL@0 esteem@0 peer@0 appear@0 phy@0 parent@0 schocom@0

schoaff@0 Germcom@0 Germaff@0 MathAff@0 MathCom@0;

! correlated uniquenesses between parallel worded items

SDQ 11 with SDQ 51 (cu1); SDQ 11 with SDQ 71 (cu2); SDQ 51 with SDQ 71 (cu3);

SDQ_25 with SDQ_35 (cu4); SDQ_25 with SDQ_39 (cu5); SDQ_35 with SDQ_39 (cu6);

SDQ 41 with SDQ 68 (cu7); SDQ 41 with SDQ 9 (cu8); SDQ 68 with SDQ 9 (cu9);

SDQ_57 with SDQ_20 (cu10); SDQ 57 with SDQ_55 (cu11); SDQ_20 with SDQ_55 (cu12);

SDQ 23 with SDQ_6 (cu13); SDQ_23 with SDQ_65 (cu14); SDQ 6 with SDQ_65 (cu15);

SDQ 4 with SDQ 27 (cu16); SDQ 4 with SDQ_16 (cu17); SDQ 27 with SDQ 16 (cu18);

SDQ_18 with SDQ 59 (cu19); SDQ_18 with SDQ_2 (cu20); SDQ 59 with SDQ 2 (cu21);

SDQ_49 with SDQ_13 (cu22); SDQ_49 with SDQ_63 (cu23); SDQ_13 with SDQ_63 (cu24);

SDQ 73 with SDQ 43 (cu25); SDQ 73 with SDQ 31 (cu26); SDQ 43 with SDQ 31 (cu27);

SDQ 47 with SDQ_75 (cu28); SDQ 47 with SDQ 33 (cu29); SDQ 75 with SDQ 33 (cu30);

SDQ_1 (1); SDQ_2 (2); SDQ_3 (3); SDQ_4 (4); SDQ_5 (5); SDQ_6 (6); SDQ_7 (7); SDQ 8 (8);

SDQ 9 (9); SDQ 10 (10); SDQ 11 (11); SDQ 12 (12);

SDQ_13 (13); SDQ_14 (14); SDQ_15 (15); SDQ_16 (16);

SDQ 17 (17); SDQ 18 (18); SDQ 19 (19); SDQ 20 (20);

SDQ_21 (21); SDQ_22 (22); SDQ_23 (23); SDQ_24 (24);

SDQ 25 (25); SDQ 26 (26); SDQ 27 (27); SDQ 28 (28);

SDQ_29 (29); SDQ_30 (30); SDQ_31 (31); SDQ_32 (32);

SDQ 33 (33); SDQ 34 (34); SDQ 35 (35); SDQ 36 (36):

SDQ_37 (37); SDQ_38 (38); SDQ_39 (39); SDQ_40 (40);

SDQ 41 (41); SDQ 42 (42); SDQ 43 (43); SDQ 44 (44);

SDQ_45 (45); SDQ_46 (46); SDQ_47 (47); SDQ_48 (48);

SDQ 49 (49); SDQ 50 (50); SDQ_51 (51); SDQ 52 (52);

SDQ_53 (53); SDQ_54 (54); SDQ_55 (55); SDQ_56 (56);

SDQ 57 (57); SDQ 58 (58); SDQ 59 (59); SDQ 60 (60);

SDQ 61 (61); SDQ 62 (62); SDQ 63 (63); SDQ 64 (64);

SDQ_65 (65); SDQ_66 (66); SDQ_67 (67); SDQ_68 (68); 
SDQ_69 (69); SDQ 70 (70); SDQ 71 (71); SDQ 72 (72);

SDQ_73 (73); SDQ_74 (74); SDQ_75 (75); SDQ_76 (76);

! invariance of variances and covariances

global-mathaff@1;

esteem with peer (a31); esteem with phys (a32); esteem with parent (a33);

esteem with schocom (a34); esteem with schoaff (a35); esteem with Germcom (a36);

esteem with Germaff (a37); esteem with mathcom (a38); esteem with mathaff (a39);

esteem with appear (a40); esteem with global (a30);

peer with phys (a41); peer with parent (a42); peer with schocom (a43);

peer with schoaff (a44); peer with Germcom (a45); peer with Germaff (a46);

peer with mathcom (a47); peer with mathaff (a48); peer with appear (a49);

peer with global (a50);

phys with parent (a51); phys with schocom (a52); phys with schoaff (a53);

phys with Germcom (a54); phys with Germaff (a55); phys with mathcom (a56);

phys with mathaff (a57); phys with appear (a58); phys with global (a59);

parent with schocom (a60); parent with schoaff (a61); parent with Germcom (a62);

parent with Germaff (a63); parent with mathcom (a64);

parent with mathaff (a65); parent with appear (a66); parent with global (a67);

schocom with schoaff (a70); schocom with Germcom (a71); schocom with Germaff (a72);

schocom with mathcom (a73); schocom with mathaff (a74); schocom with appear (a75);

schocom with global (a76);

schoaff with Germcom (a80); schoaff with Germaff (a81); schoaff with mathcom (a82);

schoaff with mathaff (a83); schoaff with appear (a84); schoaff with global (a85);

Germcom with Germaff (a90); Germcom with mathcom (a91); Germcom with mathaff (a92);

Germcom with appear (a93); Germcom with global (a94); Germaff with mathcom (a100);

Germaff with mathaff (a101); Germaff with appear (a102); Germaff with global (a103);

mathcom with mathaff (a110); mathcom with appear (a111); mathcom with global (a112);

mathaff with appear (a113); mathaff with global (a114);

appear with global (a115);

[global-mathaff@0]; 